\title{
STUDIES OF PROTEIN STRUCTURE IN SOLUTION AND PROTEIN FOLDING USING SYNCHROTRON SMALL-ANGLE X-RAY SCATTERING
}

Lingling Chen

MASTER

SLAC-Report-498

April 1997

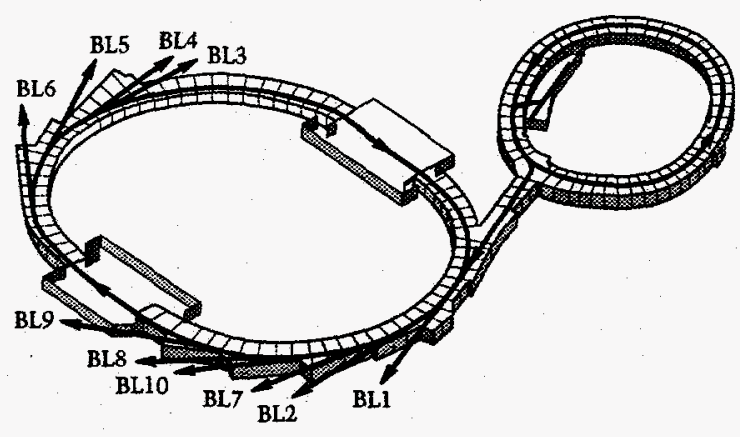

Prepared for the Department of Energy under contract number DE-AC03-76SF00515

STANFORD LINEAR ACCELERATOR CENTER

STANFORD SYNCHROTRON RADIATION LABORATORY

Stanford University - Stanford, California 
This document and the material and data contajned therein, was developed under sponsorship of the United States Government. Neither the United States nor the Department of Energy, nor the Leland Stanford Junior University, nor their employees, nor their respective contractors, subcontractors, or their employees, makes any warranty, express or implied, or assumes any liability or responsibility for accuracy, completeness or usefulness of any information, apparatus, product or process disclosed, or represents that its use will not infringe privately-owned rights. Mention of any product, its manufacturer, or suppliers shall not, nor is it intended to, imply approval, disapproval, or fitness for any particular use. A royalty-free, nonexclusive right to use and disseminate same for any purpose whatsoever, is expressly reserved to the United States and the Universitv.

\section{DISCLAIMER}

This report was prepared as an account of work sponsored by an agency of the United States Government. Neither the United States Government nor any agency thereof, nor any of their employees, makes any warranty, express or implied, or assumes any legal liability or responsibility for the accuracy, completeness, or usefulness of any information, apparatus, product, or process disclosed, or represents that its use would not infringe privately owned rights. Reference herein to any specific commercial product, process, or service by trade name, trademark, manufacturer, or otherwise does not necessarily constitute or imply its endorsement, recommendation, or favoring by the United States Government or any agency thereof. The views and opinions of authors expressed herein do not necessarily state or reflect those of the United States Government of any agency thereof. 


\section{DISCLAIMIER}

Portions of this document may be illegible in electronic image products. Images are produced from the best available original docrment. 


\title{
STUDIES OF PROTEIN STRUCTURE IN SOLUTION AND PROTEIN FOLDING USING SYNCHROTRON SMALL-ANGLE $X$-RAY SCATTERING
}

\author{
Lingling Chen \\ Stanford Linear Accelerator Center \\ Stanford Synchrotron Radiation Laboratory \\ Stanford University, Stanford, California 94309
}

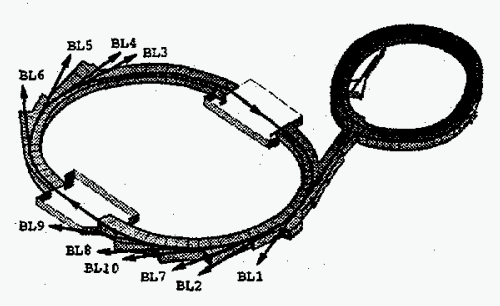

SLAC-Report-498
April 1996

Prepared for the Department of Energy under contract number DE-AC03-76SF00515 and the NIH, Biomedical Resource Technology Program,

Division of Research Resources

Printed in the United States of America. Available from the National Technical Information Service, U.S. Department of Commerce, 5285 Port Royal Road, Springfield, VA 22161

*Ph.D. Thesis Stanford University, Stanford, CA 94309 
STUDIES OF PROTEIN STRUCTURE IN SOLUTION AND PROTEIN FOLDING USING SYNCHROTRON SMALL-ANGLE X-RAY SCATTERING

\author{
A DISSERTATION \\ SUBMITTED TO THE DEPARTMENT OF CHEMISTRY \\ AND THE COMMITTEE ON GRADUATE STUDIES \\ OF STANFORD UNIVERSITY \\ IN PARTIAL FULFILLMENT OF THE REQUIREMENTS \\ FOR THE DEGREE OF \\ DOCTOR OF PHILOSOPHY
}

By

Lingling Chen

April 1996 


\section{Abstract}

Synchrotron small angle $x$-ray scattering (SAXS) has been applied to the structural study of several biological systems, including the nitrogenase complex, the heat shock cognate protein (hsc70), and lysozyme folding. The structural information revealed from the SAXS experiments is complementary to information obtained by other physical and biochemical methods, and adds to our knowledge and understanding of these systems.

Nitrogenase complex from Azotobacter vinelandii. The nitrogenase enzymatic system consists of two $\mathrm{O}_{2}$-sensitive metalloproteins: the iron protein ( $\mathrm{Fe}$ protein) and the molybdenum-iron protein (MoFe protein). The Fe protein transfers electrons from electron donors to the MoFe protein, and hydrolysis of ATP is required for the electron transfer. The MoFe protein contains a active site called FeMo cofactor where $\mathrm{N}_{2}$ reduction occurs.

To investigate the effect of nucleotide (MgATP and MgADP) binding on the structure of the Fe protein, SAXS experiments have been carried out on the wild-type and a Nif mutant (UW91, which has an alanine157 mutation to serine) Fe proteins of nitrogenase from Azotobacter vinelandii. For the wild-type protein, the binding of $\mathrm{MgATP}$ induces a significant conformational change as evidenced by a decrease of about $2.0 \AA$ in the radius of gyration $\left(\mathrm{Rg}_{\mathrm{g}}\right)$. In contrast, the binding of MgADP to the wild type Fe protein does not detectably affect $\mathrm{R}_{\mathrm{g}}$. In the absence of nucleotides, the $\mathrm{Rg}_{\mathrm{g}}$ for the UW91 mutant is indistinguishable from that of the wild type. However, unlike the wild type protein, the $\mathrm{R}_{\mathrm{g}}$ of the UW91 Fe protein is not affected by the addition of MgATP. Because the UW9] protein has a normal [4Fe-4S] cluster and MgATP binding ability but both the electron transfer and MgATP bydrolysis are completely blocked, these $\mathrm{x}$-ray scattering measurements suggest that a conformation different from that of the native state is required for the Fe protein to perform electron transfer to the MoFe protein. These results also support the hypothesis that residue alanine 157 is crucial for the iron protein to establish the electron-transfer-favored conformation induced by MgATP binding.

As a first step to study the interaction between the $\mathrm{Fe}$ protein and the MoFe protein during electron transfer, scattering data for the MoFe protein have also been taken, and the $R_{g}$ is measured to be $39.8( \pm 0.4) \AA$. A FeMo cluster, a different form of 
the FeMo cofactor, is mostly in homogeneous monomeric form in $N$-methylformamide solution, with a $R_{g}$ of $3 . f( \pm 0.4) \AA$. However, the FeMo cluster appears to highly aggregate in methyl-ethyl ketone solution.

The $70 \mathrm{kDa}$ heat shock cognate protein hsc70. The bovine heat shock cognate protein (hsc70) is a constitutively expressed member of the molecular chaperones that are necessary for cell viability in all organisms. Solution $x$-ray scattering experiments have been carried out on recombinant bovine hsc70 (with 650 amino acid residues), a $60 \mathrm{kDa}$ subfragment (residues 1-554) which has ATPase and peptide binding activities, a $44 \mathrm{kDa}$ subfragment (residues 1-386) which has only ATPase activity, and a peptide binding fragment (residues 388-554). Modeling based on the steady-state values of $R_{g}$ and $P(r)$ functions show that the ATPase and peptide binding domains are oblate fragments, while hsc70 and the $60 \mathrm{kDa}$ fragment are prolate and relatively elongated. $R_{g}$ values of hsc70 and the $60 \mathrm{kDa}$ fragments decrease significantly in the presence of MgATP relative to their values in the presence of $\mathrm{MgADP}\left(\Delta \mathrm{R}_{\mathrm{g}}-4-5 \AA\right)$; in contrast, the $\mathrm{R}_{\mathrm{g}}$ of the ATPase fragment remains essentially the same in the presence of either nucleotide. The kinetics of the change of $\mathrm{R}_{\mathrm{g}}$ for hsc70 and the $60 \mathrm{kDa}$ fragment under single-ATPase-cycle conditions show that the transition to the ATP-induced $R_{g}$ occurs significantly more rapidly than ATP hydrolysis, while the reverse transition to the larger $R_{g}$ value does not occur before product release. Altogether, the solution scattering data support a model in which a conformational change in hsc70 (presumably to the low peptide affinity state) is predicated on ATP binding, while the reverse transition is predicated on product release.

Equilibrium lysozyme unfolding. Equilibrium unfolding of hen egg lysozyme as a function of urea concentration at $\mathrm{pH} 2.9$ and $\mathrm{pH} 2.0$ has been studied by solution x-ray scattering. A difference in the cooperativity of the unfolding as monitored by the radius of gyration, $R_{g}$, is observed relative to that seen from the CD signal at $222 \mathrm{~nm}$. This suggests the existence of a third scattering species, in addition to the native and the unfolded states. A singular value decomposition (SVD) analysis was applied to the scattering curves at different urea concentrations. This analysis shows clear evidence of a third basis component in the $\mathrm{x}$-ray scattering curves, thus supporting the results of the $\mathbf{R}_{\mathbf{g}}$ and $C D$ measurements. The denaturant binding model was employed to estimate the thermodynamic parameters of denaturation for the intermediate and unfolded states. Use of these parameters to refine the SVD analysis allows us to reconstruct a scattering profile for the pure intermediate state. Simplified partially folded models based on the crystal 
structure of hen lysozyme support a working model for the intermediate whose structure may be correlated with that of the kineti intermediate found in the refolding pathway.

Kinetics of lysozyme refolding. The refolding process of lysozyme has been followed by time-resolved SAXS combined with the stopped-flow method. At the earliest measurable time ( $100 \mathrm{~ms}$ after initiation of refolding), $R_{\mathrm{g}}$ bas decreased from $22 \AA$ to $19 \AA$. A fast process has been found to occur within the deadtime of the experiment (100 $\mathrm{ms}$ ), which accounts for $>30 \%$ of the total change in $\mathrm{R}_{\mathrm{g}}$ between the unfolded and the native states. The time constants $(\tau)$ for the slow process have been derived for different refolding conditions. The biphasic kinetics suggests that a folding intermediate forms within $100 \mathrm{~ms}$ in the pathway. The forward scattering intensity $(\mathrm{I}(0))$ has been found to remain steady within experimental error throughout the refolding process, indicating that the change in $\mathrm{R}_{\mathrm{g}}$ observed is due to the conformational change in the refolding process, rather than a change in aggregation state. 


\section{Acknowledgments}

My graduate career at Stanford has been a tremendously rewarding experience. Many people have contributed in different ways to this work, and it is impossible to express my gratitude to everyone of them individually.

First, I would like to thank my research advisor, Keith Hodgson, for his generous support and encouragement throughout my research. Keith's patience and faith in my ability allowed me to develop independent thinking, and further my interest in biophysical field. I appreciate the opportunities he has provided to visit different laboratories including Photon Factory in Japan, and to present the projects that I worked on at conferences locally and internationally. I would like to acknowledge Seb Doniach for his insights which provide a step further in understanding experimental results. Seb's scientific enthusiasm and innovative ideas have been the inspirations to me during my research. I feel privileged to work under both Keith and Seb.

I would like to thank Britt Hedman and Pat Frank, two marvelous people in the Hodgson group. They are the resources that I can always rely on whenever I encountered difficulties, scientifically and personally. I am particularly grateful to Britt for proof reading this thesis, and for her perspectives and being a role model as a female scientist. I have benefited a great deal from Pat on a daily basis. He taught me step by step purification of nitrogenase, and is never tired of answering my constant questions. Pat also serves as my English tutor, commenting on my papers and part of this thesis, as well as teaching me American culture and history. I still owe him a jar of jelly worms from Xiamen (Fujian)!

Hiro Tsuruta and David Eliezer introduced me to small-angle x-ray scattering, and we had been working closely over the years. Hiro had always been the responsible personnel when I had emergency at the beamline. I would like to thank both of them for sharing with me the stressful and 'rewarding' experience with synchrotron experiments. It is a great pleasure to work with them. Dan Segel joined the group two years ago, and has made immediate contributions to SAXS. Dan is always happy to discuss any subjects and to offer helps. I would like to thank him for reading part of this thesis, for his American idioms, and most importantly for his friendship. I wish both him and Mike Bada, who just joined the group a few days ago, the best in their future experiments and successful graduate careers. 
I would like to thank the rest of Hodgson members, past and present, Heather Bufford, Jason Chen, Jane DeWitt, Isaac Liu, Kristin Musgrave, Kent Nakagawa, Susan Shadle, Chrisie Stanfel, Grace Tan, Trevor Tyson, Erik Wasinger, Kendra Williams, Tami Westre, Holly Zhang, for creating a most harmonious and pleasant working environment. Being my neighbor, Chrisie has 'endured' the most of my complaints, and has always been willing to help me through those difficult moments. I have had a wonderful time working with members of Boxer group, sharing chemicals and instruments, and enjoying their great barbecue parties.

I am thankful to have the opportunity to work collaboratively with Burgess and McKay laboratories. Not only did they supply the high quality samples, but they also worked closely with me days and nights on the beamline, responsible for any sample emergency. Their pleasant cooperation has kept our projects going as smoothly as they could be. I would like to thank Dave McKay for his critical scientific attitude and his encouragement.

The SSRL staff have provided invaluable technical support. The x-ray group made extra efforts working on a stable and focused beam, and the SSRL machine shop did an excellent job on making sample cells and beamline components.

I would like to give special thanks to my previous advisors, Prof. Hui-Lin Wan, and Prof. Qi-Rui Tsai at Xiamen University, China. Prof. Wan guided me to the field of Physical Chemistry, and showed me the significance of doing research. I am indebted to his constant support, both psychological and academic, of my education at Xiamen University. I am grateful for their visits during my stay at Stanford.

A great deal of credits go to friends who have provided encouragement as well as entertainment all the way. I would like to thank my English tutor Jane Rebber who introduced me to American culture, and helped me to improve my English. Jian-Yi Lin and Leping $\mathrm{Li}$ are among the people who helped me to settle at Stanford. Ming-Qian Ma serves as my mentor in various aspects. Friends, far and close, Xiaoping Song, Jiahua Che, Kaiqin Lao, Huilin Zhou ... are truly dependable. A special thank goes to the Bromberg family, particularly our little friends, Leah, Luke, Nathaniel and Paulina for their hospitality. I will always cherish the friendships we share. 
I would like to express my deep appreciation of my parents. My late father, Bingnan Chen, had always been supportive for higher education, and stayed by me every. step of the way along my career path. I would not reach the doctorate stage without him. I am deeply indebted to my mother, Xiuli Liao, for her giving me the freedom to study abroad and pursue my goal. Her love, patience and understanding have allowed me to concentrate on my research. It is a great comfort to have her around! I owe my parents more than I could express. I would also like to thank my brothers, Huiqing and Ningxiong, and sisters-in-law, Lijuan and Qinhua, for their unconditional and longdistance support, and warm encouragement.

Finally, I feel very fortunate to have Xiaohua Huang, my husband, sharing with me the ups and downs for the past three years at Stanford. Xiaohua's unique research style has inspired me to keep my mind open, and his critique of my work has motivated me for different and better approaches. Without his contributions to the projects that I have been working on, this thesis would not come out as it is. In addition, I would like to thank him for his support and company during the beamtime - without him, I would not be able to work continuously for 20 hours a day for almost a week. His love and companion are what make the completion of this thesis possible. 
To my Mother, Xiuli Liao, and the memory of my Father, Bingnan Chen 


\section{Table of Contents}

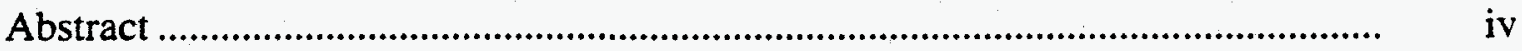

Acknowledgments ............................................................................................. vii

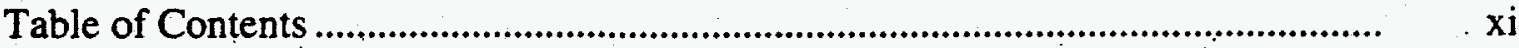

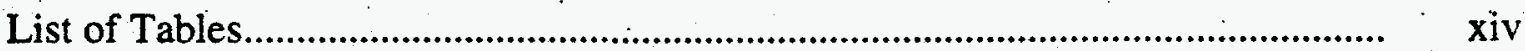

List of Figures

List of Abbreviations...................................................................................... xviii

Chapter 1 Introduction and Background........................................................... 1

1.1. Scope and Organization of This Thesis.............................................................. 2

1.2. Overview of X-ray Scattering Theory................................................................ 3

1.3. The Small-angle $\mathrm{X}$-ray Scattering Technique ..................................................... 5

1.3.1. Guinier Approximation ................................................................. 5

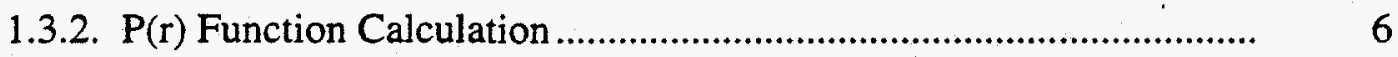

1.3.3. Debye Formula ............................................................................ 7

1.4. Information Content from Small-angle X-ray Scattering..................................... 8

1.4.1. Size Information - Radius of Gyration .............................................. 8

1.4.2. Molecular Weight (MW) .................................................................

1.4.3. Shape Information $-P(r)$ Function and Dmax ..................................

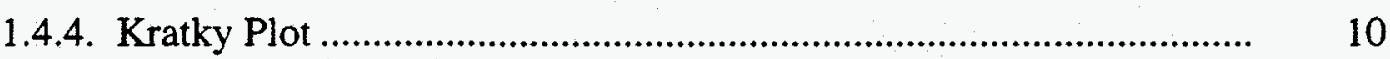

1.5. General Experimental Aspects .................................................................... 11

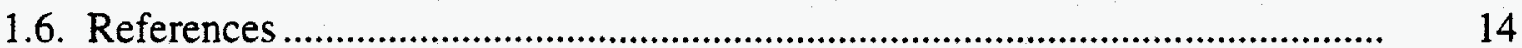

Chapter 2 Solution Small-angle X-ray Scattering Study of the Components of the Azotobacter vinelandii Nitrogenase Enzyme System

2.1. Introduction and Background....................................................................... 17

2.1.1. The Fe Protein .......................................................................... 17

2.1.2. The MoFe Protein.......................................................................... 20

2.1.3. The FeMo Cofactor ....................................................................... 20

2.1.4. The FeMo Cluster.......................................................................... 22

2.2. Experimental Procedures....................................................................... 23

2.2.1. The Fe Protein ................................................................................ 
2.2.2. The MoFe Protein

2.2.3. The FeMo Cluster................................................................. 26

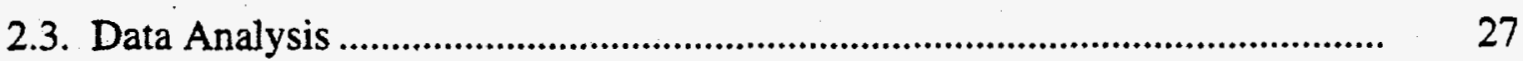

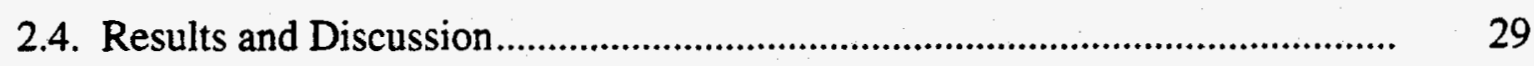

2.4.1. The Fe Protein ......................................................................... 29

2.4.2. The MoFe Protein................................................................... 37

2.4.3. The FeMo Cluster....................................................................... 39

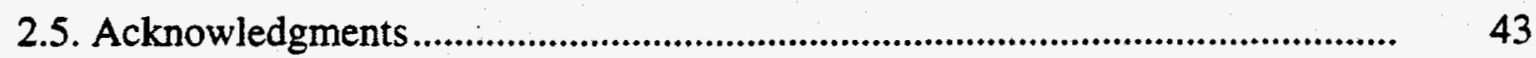

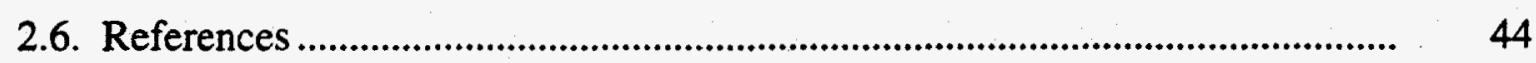

Chapter 3 Solution Small-angle X-ray Scattering Study of the Molecular Chaperone hsc70 and Its Subfragments.

3.1. Introduction and Background............................................................. 48

3.2. Experimental Procedures.................................................................... 52

3.2.1. Sample Preparations................................................................ 52

3.2.2. X-ray Scattering Data Collection ................................................ 55

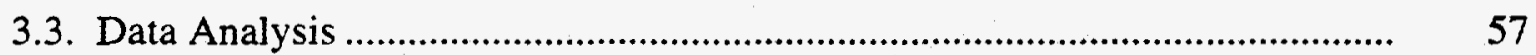

3.3.1. Data Reduction and Analysis ..................................................... 57

3.3.2. Computing Scattering Curves from Protein Coordinates.................. 58

3.3.3. Computing Scattering Curves from Some Simple Triaxial Bodies ... 58

3.4. Results

3.4.1. The Static Measurements ......................................................... $\quad 62$

3.4.1.1. A Standard Globular Protein - BSA ................................ . . 62

3.4.1.2. The Guinier Analysis on hsc70 and Its Subfragements ..... 65

3.4.1.3. The $P(r)$ Function Analysis on hsc70 and Its Subfragments 77

3.4.2. Kinetic Measurements.................................................................... 81

3.4.3. Modeling Based on $\mathrm{P}(\mathrm{r})$ Functions.............................................. 84

3.4.3.1. Modeling the ATPase and the Peptide-binding Domains ... 84

3.4.3.2. Modeling the $60 \mathrm{kDa}$ Fragment....................................... 86

3.4.3.3. Modeling the Full-length hsc70 ........................................ 91

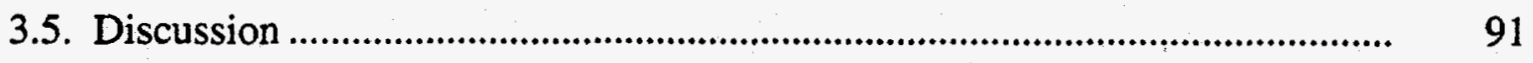

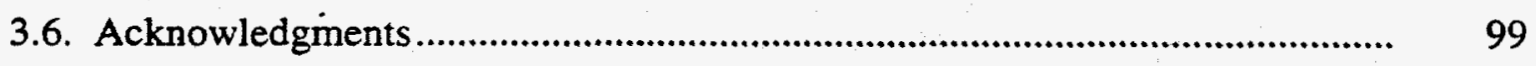

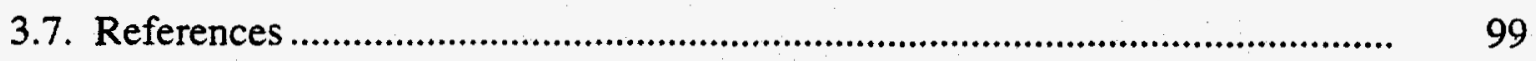


Chapter 4 Equilibrium Studies of Lysozyme Unfolding by Solution X-ray Scattering: Evidence for A Stable Folding Intermediate.

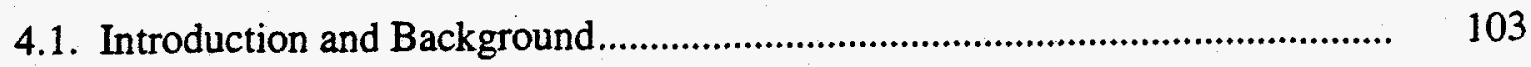

4.2. Experimental Procedures........................................................................... 108

4.2.1. Sample Preparations .................................................................... 108

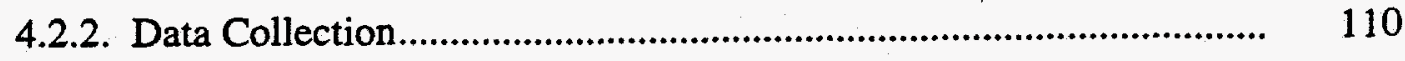

4.3. Data Analysis ....................................................................................... 111

4.3.1. Data Reduction and Analysis .......................................................... 111

4.3.2. Thermodynamic Analysis of the Denaturation Curve........................ 112

4.3.3. Models of Denaturant Effects.............................................................. 113

4.3.4. Singular Value Decomposition (SVD)............................................ 115

4.4. Results

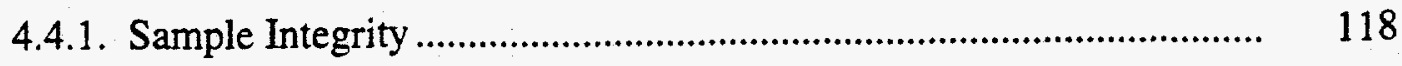

4.4.2. The Native State ............................................................................... 119

4.4.3. The Unfolded State......................................................................... 126

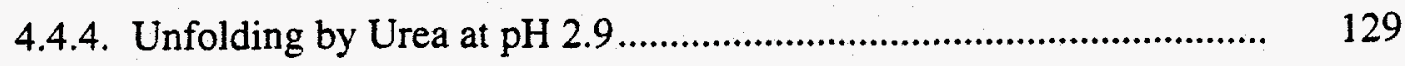

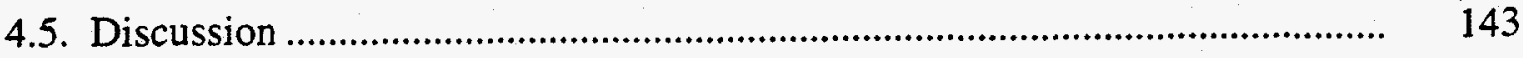

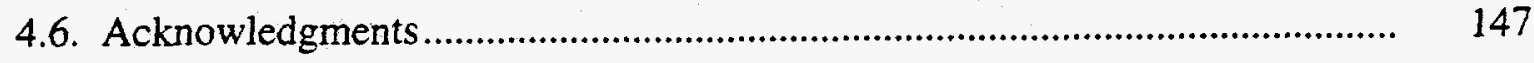

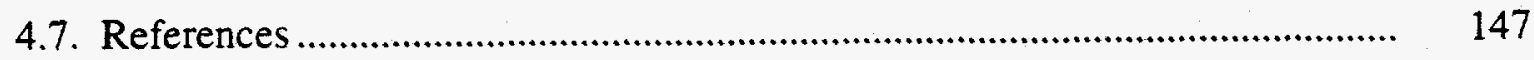

Chapter 5 Time-resolved Small-angle X-ray Scattering Studies of Lysozyme Refolding.

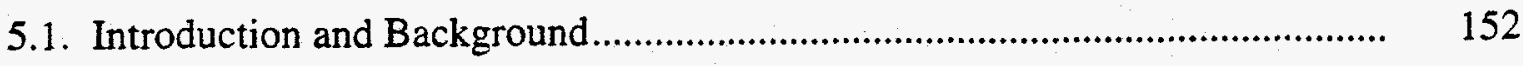

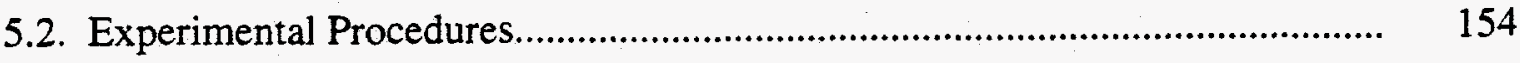

5.2.1. Sample Preparations ..................................................................... 154

5.2.2. Data Collection .............................................................................. 155

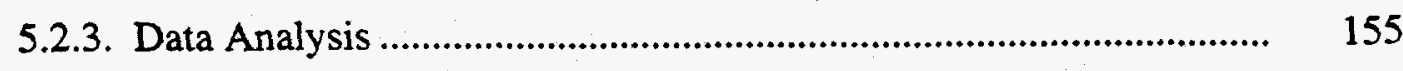

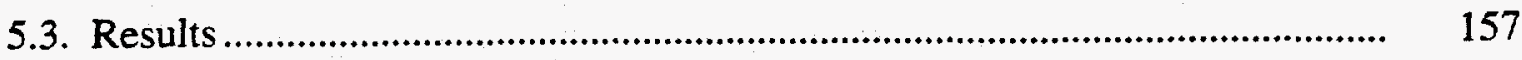

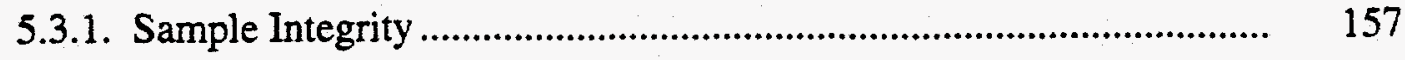

5.3.2. The Initial and Final States............................................................

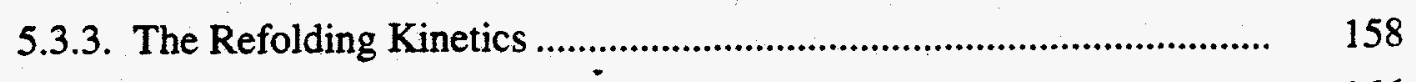

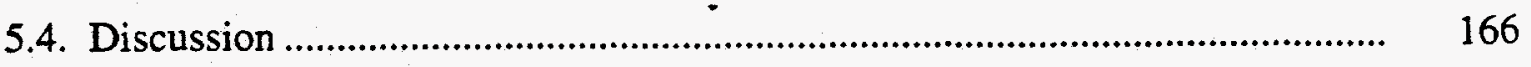

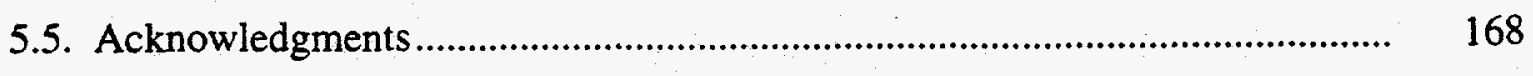

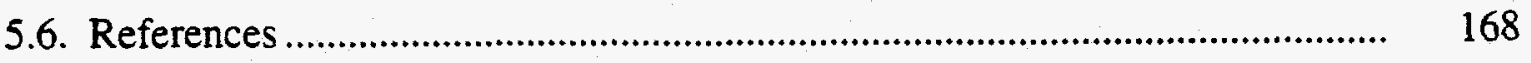




\section{List of Tables}

\section{Chapter 2}

Table 2.1. $\quad \mathrm{Rg}$ for the Fe Protein ..........................................................................

Table 2.2. $\mathrm{Rg}_{\mathrm{g}}$ of the FeMo Cluster and the Fitting Range ..................................... . 42

\section{Chapter 3}

Table 3.1. Rg for hsc70 and Its Subfragments .........................................................

Table 3.2. $R_{g}$ for hsc70 and Its Subfragments under Different

Nucleotide Conditions........................................................................

\section{Chapter 4}

Table 4.1. Parameters for Simultaneous Fits to the $\mathrm{CD}(222 \mathrm{~nm})$ and $\mathrm{Rg}$ Unfolding Curves.

Table 4.2. $\mathrm{Rg}_{\mathrm{g}}$ for the Fragments of Lysozyme

\section{Chapter 5}

Table 5.1. Kinetic Parameters for Lysozyme Refolding 


\section{List of Figures}

\section{Chapter 1}

Figure 1.1. Schematic Drawing of SAXS Setup

\section{Chapter 2}

Figure 2.1. Schematic Representation of the Mo Nitrogenase

Figure 2.2. Structural Model for the FeMo Cofactor.

Figure 2.3. Typical Guinier Plot for the Fe Protein........................................... 30

Figure 2.4. Plot of $\mathrm{R}_{\mathrm{g}} v s$ [Fe Protein] .......................................................... 31

Figure 2.5. Plot of $\mathrm{Rg} v s \quad[\mathrm{MoFe}$ Protein] ........................................................... 38

Figure 2.6. Guinier Plot for the FeMo Cluster ................................................. 41

\section{Chapter 3}

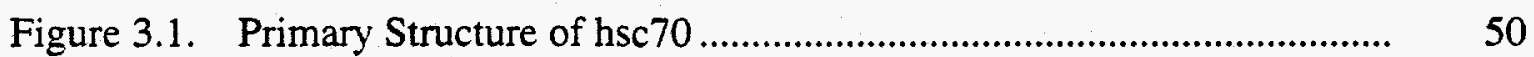

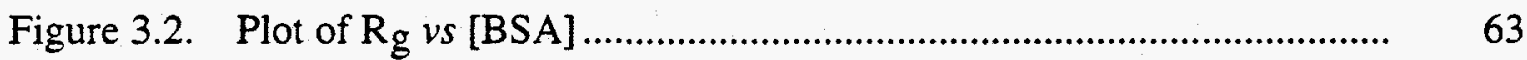

Figure 3.3. Comparison of Scattering Curves of BSA Using the Linear and the Quadrant Detectors........................................................... 64

Figure 3.4. Combined Scattering Data for BSA and the $P(r)$ Function................. 66

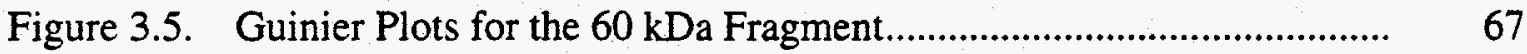

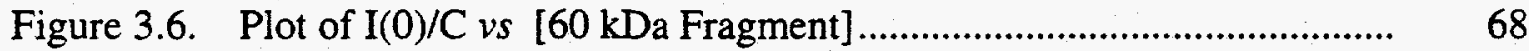

Figure 3.7. Plots of $\mathrm{Rg}_{\mathrm{g}}$ vs [hsc70], $\mathrm{Rg}$ vs [60 $\mathrm{kDa}$ Fragment], $\mathrm{R}_{\mathrm{g}}$ vs [ATPase Fragment], and $\mathrm{R}_{\mathrm{g}} v s$ [Peptide Binding Domain] ................ 71

Figure 3.8. $\mathrm{P}(\mathrm{r})$ for hsc 70 and the $60 \mathrm{kDa}$ Fragment......................................... 79

Figure 3.9. $\mathrm{P}(\mathrm{r})$ for the ATPase and Peptide Binding Domains.......................... $\quad 80$

Figure 3.10. Kinetics of Changes in $\mathrm{R}_{\mathrm{g}}$ in the ATPase Cycle of hsc70 and the $60 \mathrm{kDa}$ Fragment

Figure 3.11. Comparison of $P(r)$ Functions of the ATPase Domain and a Box Model and the Superposition of the Box with a Space Filling Model of the ATPase Domain

Figure 3.12. Comparison of $P(r)$ Functions of the Peptide Binding

Domain and the Box Model .... 
Figure 3.13. $\mathrm{P}(\mathrm{r})$ Functions for the Monomeric Box Models for the $60 \mathrm{kDa}$ Fragment

Figure 3. 4. P(r) Functions for the "Partial Dimer" Box Models for the $60 \mathrm{kDa}$ Fragment.

Figure 3.15. $\mathrm{P}(\mathrm{r})$ Functions for the Box Models for hsc70

Figure 3.16. Mechanisms for the ATP-induced Conformational Change in the $60 \mathrm{kDa}$ Fragment.

\section{Chapter 4}

Figure 4.1. Structure of Hen Lysozyme ............................................................. 106

Figure 4.2. Comparison of $\mathrm{X}$-ray Absorption by $\mathrm{GdmHCl}$ and Urea ..................... $\quad 109$

Figure 4.3. Plot of $\mathrm{R}_{\mathrm{g}} v s$ [Lysozyme] ............................................................ 120

Figure 4.4. Comparison of Scattering Curves at Different Lysozyme Concentrations.

Figure 4.5. Comparison of the Experimental and the Calculated $\mathrm{P}(\mathrm{r})$ Functions for Lysozyme.

Figure 4.6. Comparison of the Experimental and the Calculated Kratky Plots for Lysozyme.

Figure 4.7. Comparison of Scattering Curves of Lysozyme at Different $\mathrm{pH}$ Solutions

Figure 4.8. Plot of $\mathrm{Rg}_{\mathrm{g}} v$ [Heparin].

Figure 4.9. Kratky Plot and $\mathrm{P}(\mathrm{r})$ Function for the Unfolded Lysozyme

Figure 4.10. Plot of $\mathrm{I}(0) / \mathrm{C}$ vs [Urea] for Lysozyme

Figure 4.11. Unfolding Transition Curves by $\mathrm{CD}$ and $\mathrm{Rg}$ at $\mathrm{pH} 2.9, \mathrm{pH} 2.0$ and the Fits to Models

Figure 4.12. Plot of Population of Native, Intermediate and Unfolded States vs [Urea].

Figure 4.13. Comparison of Scattering Data and the Three-Component SVD Approximation.

Figure 4.14. Three Orthogonal Basis Scattering Functions

Figure 4.15. Coefficients for the Three Basis Functions and the Fits.

Figure 4.16. SVD Constructed Kratky Plot for the Intermediate State and the $P(r)$ Function 


\section{Chapter 5}

Figure 5.1. Comparison of Scattering Curves of Stopped-flow Data and the Control Data..

Figure 5.2. Comparison of Data Statistics with Different Time Duration in a Stopped-flow Experiment.......................................................... 160

Figure 5.3. Time-course of $\mathrm{Rg}_{\mathrm{g}}$ in the Refolding of Lysozyme ............................. 162

Figure 5.4. Time-course of $\mathrm{R}_{\mathrm{g}}$ in a Control Experiment........................................ 163

Figure 5.5. Kratky Plots of the First and the Last Time-Frame Data in a Stopped-flow Experiment.................................................................. 165 


\section{List of Abbreviations}

\begin{tabular}{|c|c|}
\hline$\AA$ & Ångström \\
\hline ADP & adenosine diphosphate \\
\hline AMPPNP & 5 -adenylyl- $\beta, \gamma$-imidodiphosphate \\
\hline ANS & 8-anilinonapthalene-1-sulfonate \\
\hline ATP & adenosine triphosphate \\
\hline $\mathrm{CD}$ & circular dichroism \\
\hline $\mathrm{Da}$ & dalton \\
\hline DOE & Department of Energy \\
\hline $\mathrm{GdmHCl}$ & guanidinium hydrochloride \\
\hline hsc70 & $70 \mathrm{kDa}$ heat-shock cognate \\
\hline $\mathrm{I}(0)$ & forward scattering intensity at zero scattering angle \\
\hline MEK & methyl-ethyl ketone \\
\hline MW & molecular weight \\
\hline $\mathrm{NIH}$ & National Institutes of Health \\
\hline NMF & $N$-methyl formamide \\
\hline$R_{g}$ & radius of gyration \\
\hline SAXS & small-angle $x$-ray scattering \\
\hline SSRL & Stanford Synchrotron Radiation Laboratory \\
\hline SVD & singular value decomposition \\
\hline
\end{tabular}




\section{Chapter 1}

Introduction and Background 


\subsection{Scope and Organization of This Thesis}

This dissertation focuses on the applications of synchrotron small-angle $\mathrm{x}$-ray scattering (SAXS) to the study of conformation and structure of biological systems in solution. Chapter 1 provides a general introduction to the small-angle $x$-ray scattering technique, including a brief theoretical background, information content from SAXS, and experimental considerations. This is followed by four chapters describing detailed experiments, data analysis and results, focusing on the studies of three topics - the nitrogenase enzymatic system, the heat-shock cognate protein hsc70, and lysozyme folding. A brief introduction is provided in each of these chapters focusing on the aspects of the project to be studied. Chapter 2 presents the results of conformational studies of the Fe protein, the MoFe protein and a FeMo cluster of Azotobactor vinelandii nitrogenase. Chapter 3 describes the structural studies of a chaperone, hsc70, and its subfragments, concentrating on the development of low resolution models for hsc70 and the kinetics of conformational change in the ATPase cycle. Both Chapter 4 and Chapter 5 concerns the application of SAXS to studies of protein folding. Results of equilibrium unfolding of lysozyme are given in Chapter 4, and those of a time-resolved SAXS study of lysozyme refolding kinetics in Chapter 5. 


\subsection{Overview of $\mathrm{X}$-ray Scattering Theory}

When $\mathrm{x}$-rays pass through matter, they interact with both electrons and nuclei. However, as we can see below, the scattering of $x$-rays by matter comes primarily from the interaction of $x$-rays with electrons. This is because the scattering length of a nucleus is less than $10^{-3}$ of that of an electron so that the nuclear scattering can be neglected.

Incident radiation can be represented by a plane monochromatic incident wave: $\overrightarrow{\mathrm{E}}(\overrightarrow{\mathrm{r}})=\mathrm{E}_{0} \exp \left(2 \pi \overrightarrow{\mathrm{k}}_{0} \bullet \overrightarrow{\mathrm{r}}-\omega \mathrm{t}\right)$, where $\overrightarrow{\mathrm{E}}$ is the electric field at position $\overrightarrow{\mathrm{r}}$ with maximal amplitude $E_{0} ; \vec{k}_{0}$ is the unit vector of the incident wave; and $\omega$ is the angular frequency. Suppose an incident $\mathrm{x}$-ray beam $\left(\overrightarrow{\mathrm{k}}_{0}\right)$ impinges on a free electron positioned at the origin $O$, then the amplitude of the scattering wave $(\vec{k})$ is given by the following equation (Landau \& Lifshitz, 1987):

$$
E_{s}=-E_{0} \frac{e^{2}}{m c^{2}} \frac{1}{r} \sin \psi
$$

where $\mathrm{e}$ and $\mathrm{m}$ are the charge and mass of the electron; $\mathrm{c}$ is the velocity of light; and $\psi$ is the angle between the scattering beam and the direction of acceleration of the electron. Note that the phase of the scattering wave is shifted by $180^{\circ}$ relatively to the incident beam.

For small-angle scattering, $\psi=90^{\circ}$. If $b_{x}=e^{2} m^{-1} c^{-2} \sin \psi=e^{2} m^{-1} c^{-2}=r_{0}$, where $r_{0}=2.82 \times 10^{-13} \mathrm{~cm}$ - the Thomson electron radius, then $b_{x}$ is called the $x$-ray scattering length of a free electron. The scattering cross section is defined as $i_{x}=b_{x}^{2}=r_{0}^{2} \sin ^{2} \psi=r_{0}^{2}$. For a single electron, $i_{x}=7.96 \times 10^{-26} \mathrm{~cm}^{2}$. From the above, it is thus clear that the scattering length is inversely proportional to the mass of the scatterer, and that the scattering amplitude by nuclei, which are more than $10^{3}$ times heavier than electrons is negligible.

Now consider a free electron positioned at $\vec{r}$. The scattering wave becomes $E(\vec{S}) e^{2 \pi i \vec{S} \bullet \vec{r}}$, where $E(\vec{S})$ is the electric field of scattered radiation by a free electron at the origin with the amplitude $E_{S}$ as defined in equation [1], $\vec{S}=\vec{k}-\vec{k}_{0}$, where $|\vec{S}|=2 \sin \theta / \lambda$, and $\lambda$ is the wavelength. The structure factor, $F(\vec{S})$, is defined as the ratio of the radiation scattered by any real sample to that scattered by a free electron at the origin. In the case of a free electron at $\overrightarrow{\mathrm{r}}, F(\overrightarrow{\mathrm{S}})=\mathrm{e}^{2 \pi \mathrm{i} \cdot \overrightarrow{\mathrm{I}}}$. Because electrons are generally not localized, electron 
density $\rho(\bar{r})$ in a volume element $d \vec{r}$ is used instead. Then the structure factor becomes $F(\vec{S})=\rho(\vec{r}) e^{2 \pi i \vec{S} \cdot \vec{r}} d \vec{r}$

For a sample (an atom or a molecule, for example) with a continuous electron density distribution, the structure factor is an integral over the entire sample:

$$
F(\vec{S})=\int \rho(\vec{r}) e^{2 \pi i \vec{S} \bullet \vec{r}} d \vec{r}
$$

Note that usually the energy of the incident $x$-rays is much higher than the binding energy of the electrons so that electrons in the sample can be approximated as free electrons. The structure factor $F(\bar{S})$, and the electron density $\rho(\bar{r})$, are a Fourier pair.

$$
\rho(\overline{\mathrm{r}})=(1 / \mathrm{V}) \int \mathrm{F}(\overrightarrow{\mathrm{S}}) \mathrm{e}^{-2 \pi \mathrm{i} \overrightarrow{\mathrm{S}} \bullet \overrightarrow{\mathrm{r}}} \mathrm{d} \overrightarrow{\mathrm{S}}
$$

where $\mathrm{V}$ is the volume of the sample.

When the sample considered is an atom, the structure factor can be written as $F(\vec{S})=f_{a} e^{2 \pi i \vec{S} \bullet \bar{r}}$, where $f_{a}$ is the atomic structure factor of atom $a$.

The above treatment is also valid for scattering of macromolecules in solution. However, the electron density, $\rho(\vec{r})$, is replaced by the excess electron density $\rho_{\text {ex }}(\vec{r})$, $\rho_{\text {ex }}(\bar{r})=\rho(\bar{r})_{\text {solute }}-\rho(\bar{r})_{\text {solvent }}=\rho(\vec{r})_{\text {solute }}-\bar{\rho}_{\text {solvent }}$, where $\rho(\vec{r})_{\text {solute }}$ and $\rho(\bar{r})_{\text {solvent }}$ are the electron density of solute (macromolecule) and solvent, respectively. $\rho(\vec{r})_{\text {solvent }}$ can be approximated by the average value $\bar{\rho}_{\text {solvent }}$.

Experimentally, it is the scattering intensity $I(\overline{\mathbf{S}})\left(\mathrm{I}(\overline{\mathrm{S}})=\mathrm{F}(\overline{\mathrm{S}}) \mathrm{F}^{*}(\overrightarrow{\mathrm{S}})\right)$ that is measured. The Fourier transform of $I(\vec{S})$ is called the Patterson function, $P(\vec{r}), P(\vec{r})$ is a self-convolution function: $P(\vec{r})=F^{-1}(I(\vec{S}))=F^{-1}\left(F(\vec{S}) F^{*}(\vec{S})\right)=\rho(\vec{r}) * \rho(-\vec{r})$. Like the Fourier pair of $F(\bar{S})$ and $\rho(\vec{r})$, the scattering intensity and the Patterson function form another Fourier pair:

$$
\begin{aligned}
& I(\bar{S})=\int P(\vec{r}) e^{2 \pi i \bar{S} \bullet \vec{r}} d \vec{r} \\
& P(\vec{r})=(1 / V) \int I(\vec{S}) e^{-2 \pi i \vec{S} \bullet \vec{r}} d \vec{S}
\end{aligned}
$$




\subsection{The Small-angle $X$-ray Scattering Technique}

Small-angle $\mathrm{x}$-ray scattering has become a standard experimental technique to study structural features of matter in the areas of condensed matter physics, polymer science, metallurgy and biophysics. The dimensions of the particles concerned range from $10 \AA$ to $1000 \AA$. Since the $\mathrm{x}$-ray wavelength $(1-2 \AA)$ is rather small compared to the size of the scatterers, significant scattering is only observed at small angular region. The term, smallangle $\mathrm{x}$-ray scattering (SAXS), is thus derived. The typical angular range is about a few degrees in scattering angle.

\subsubsection{Guinier Approximation}

In solution, the sample is isotropic, i.e. each molecule can assume every possible orientation. The actual observed scattering intensity is the spatially averaged $\mathrm{I}(\overrightarrow{\mathrm{S}})$.

$$
\mathrm{I}(\mathrm{S})=\langle\mathrm{I}(\overrightarrow{\mathrm{S}})\rangle=\int_{\Omega} \mathrm{I}(\overrightarrow{\mathrm{S}}) \mathrm{d} \Omega=4 \pi \int_{0}^{\infty} \mathrm{P}(\mathrm{r}) \frac{\sin (2 \pi \mathrm{Sr})}{2 \pi \mathrm{Sr}} \mathrm{dr}
$$

where $\Omega$ is the solid angle, and $\mathrm{P}(\mathrm{r})=\mathrm{r}^{2}\langle\mathrm{P}(\overrightarrow{\mathrm{r}})\rangle . \mathrm{P}(\mathrm{r})$ is called the distance distribution function.

The scattering intensity $\mathrm{I}(\mathrm{S})$ can be further simplified, by substituting the Mclaurin series, $\sin (2 \pi \mathrm{Sr}) / 2 \pi \mathrm{Sr}=1-(2 \pi \mathrm{Sr})^{2} / 6+(2 \pi \mathrm{Sr})^{4} / 120-\cdots$, into equation [4] and restricting to the first two terms for the small angle region where $2 \pi S R_{g}<1$, then:

$$
I(S)=I(0)\left(1-4 \pi^{2} S^{2} R_{g}^{2} / 3\right)
$$

or

$$
\begin{aligned}
& I(S)=I(0) \exp \left(-4 \pi^{2} S^{2} R_{g}^{2} / 3\right) \\
& L n I(S)=\operatorname{LnI}(0)-4 / 3 \pi^{2} R_{g}^{2} S^{2}
\end{aligned}
$$

where 


$$
\begin{aligned}
& I(0)=4 \pi \int_{0}^{\infty} P(r) d r \\
& R_{g}^{2}=\frac{1}{2} \int_{0}^{\infty} P(r) r^{2} d r / \int_{0}^{\infty} P(r) d r
\end{aligned}
$$

Equations [6a] - [6b] are different representations of the Guinier approximation (Guiner \& Fournet, 1955), and are valid only in the small angle region, i.e. $2 \pi S R_{g}<1$. The forward scattering intensity $\mathrm{I}(0)$ can be related to the total scattering length of the particle, and $\mathrm{Rg}_{\mathrm{g}}$ is the radius of gyration of the particle (molecule) about its electron density center (Glatter \& Kratky, 1982).

The Guinier approximation states that in the small angle region, the scattering intensity $\mathrm{I}(\mathrm{S})$ can be approximated as a Gaussian distribution, and the width of the distribution is related to the radius of gyration of the particle. The scattering curve for a small particle with a small $\mathrm{R}_{\mathrm{g}}$ is broad, while the curve for a large particle is narrow.

\subsection{2. $\quad \mathbf{P}(\mathbf{r})$ Function Calculation}

The $P(r)$ function in solution scattering is similar to the Patterson function $P(\vec{r})$ in crystallography (see Section 1.3.1 for the definition of the $P(r)$ function). The $P(r)$ function describes the distribution of distances between the volume elements within the particle, and thus provides information regarding the geometry of the particle.

In principle, the $\mathrm{P}(\mathrm{r})$ function can be calculated directly by Fourier transform of the scattering intensity $\mathrm{I}(\mathrm{S})$ (refer to equation [3b]). However, in reality the scattering intensity $\mathrm{I}(\mathrm{S})$ can not be taken at both the infinitely large angular region $(\mathrm{S} \rightarrow \infty)$ and the small angle region ( $\mathrm{S} \rightarrow 0)$. This problem is called the termination effect. To reduce the termination effect in performing direct Fourier transform of $I(S), I(S)$ is extrapolated to $S=0$ by the Guinier approximation, and to the large angular range by Porod's law (Glatter \& Kratky, 1982).

Another approach to calculate the $P(r)$ function is by the indirect Fourier transformation method (Glatter \& Kratky, 1982). Briefly, the $P(r)$ function is approximated as a linear combination of a series of orthogonal functions $\varphi_{U}(r)$ in the range 
of $0 \ll r \ll D$ with $D$ being the maximal dimension of the particle: $P_{A}(r)=\sum_{v=1}^{N} c_{v} \varphi_{v}(r)$, $P_{A}(r) \sim P(r)$. This function is subjected to a series of integral transformations $\hat{T}_{1}-\hat{T}_{4}$, and the resultant function $\chi_{v}(S)=\hat{T}_{4} \hat{T}_{3} \hat{T}_{2} \hat{T}_{1}\left[P_{A}(r)\right]$ is compared with the experimental $\mathrm{I}(\mathrm{S})$. The coefficients $c_{v}$ are determined by a weighted least squares approximation.

The indirect Fourier transform algorithm is implemented in the program GNOM (Semenyuk \& Svergun, 1991). The $P(r)$ functions in this dissertation are all calculated with the indirect Fourier transform method using GNOM.

\subsubsection{Debye Formula}

Instead of using a continuous electron density $\rho(\widetilde{r})$ to represent the particle, atoms in the particle can be treated as discrete scattering ensembles. The scattering length of the particle with $N$ atoms can be written as: $F(\vec{S})=\sum_{i=1}^{N} f_{a, i} e^{2 \pi i \vec{S} \vec{T}}$, where $f_{a, i}$ is the atomic structure factor for atom $i$. The scattering intensity after averaging over all the orientations is:

$$
I(S)=\sum_{i=1 j=1}^{N} \sum_{a, j}^{N} f_{a, j} \frac{\sin \left(2 \pi S r_{i j}\right)}{2 \pi S r_{i j}}
$$

where $r_{j j}$ is the distance between atoms $i$ and $j$. Equation [9] is the Debye formula (Debye, 1915). Debye formula is very useful in the calculation of the scattering curve when the structure of the particle is known.

However, in solution scattering of biomolecules, solvent effect should be taken into account. To do this, the solvent inaccessible volume of the biomolecule needs to be evaluated. In the cube method (Fedorov \& Denesyuk, 1978), the biomolecule is placed in a parallelepiped which is subdivided into small cubes with edges of $0.5-1.5 \AA$. The boundary of the biomolecule is determined by examining whether each cube belongs to the biomolecule or to the solvent (Hubbard, 1987). A more complicated method is to calculate the electron envelope of the macromolecule, and this algorithm is implemented in the program CRYSOL17 (Svergun, et al., 1995). Calculations of the scattering intensity in this dissertation are performed using the program CRYSOL17. 


\subsection{Information Content from Small-angle X-ray Scattering}

Various structural information can be obtained from small-angle x-ray scattering data (Feigin \& Svergun, 1987; Glatter \& Kratky, 1982). Information including structural parameters derived in this dissertation is summarized in the following.

\subsubsection{Size Information - Radius of Gyration}

The radius of gyration $\left(\mathrm{Rg}_{\mathrm{g}}\right)$ can be obtained directly from the scattering data by the Guinier approximation [6]. The maximal angular range $S_{\max }$ is constrained to the Guinier region, $2 \pi \mathrm{SRg}<1$. For globular-shaped protein molecules, it has been found that the linear region of $\mathrm{LnI}(\mathrm{S})$ vs. $\mathrm{S}^{2}$ in equation [6b] is extended outside the Guinier region, and a region of $2 \pi \mathrm{SRg}<1.3$ has been used to improve data fitting statistics (Olah, et al., 1993). For elongated molecules, a deviation from the Guinier region will introduce systematic errors in $\mathrm{Rg}$ calculations. (See the heat-shock proteins in Chapter 3 for an example.) The minimal $\mathrm{S}$ is typically limited by the experimental setup. Parasitic scattering mainly from slits dominates the smallest resolvable angular range. A reliable $S_{\min }$ where parasitic scattering effects become negligible can be determined experimentally. $S_{\min }$ is also related to the distance between the sample and the detector (camera length); the longer the distance the smaller the $S_{\min }$ that can be achieved.

To minimize the interparticle interference (Glatter \& Kratky, 1982), scattering curves are taken over several protein concentrations. $R_{g}$ is then obtained by an extrapolation of apparent $\mathrm{Rgs}$ to zero protein concentration.

The radius of gyration can also be calculated from the distance distribution function $(P(r)$ according to equation [8]. The advantage of this approach is that the whole scattering curve including the Guinier region is used: the $P(r)$ function is obtained using I(S) over the entire $S$ range available. Since most interference, such as interparticle interference and contamination of large aggregates, distorts the scattering intensity predominantly at the small angle region within the Guinier region, by not restricting to the use of small angular region, this method should minimize the interference effect and give a better estimate of $R_{g}$ (Glatter \& Kratky, 1982). 
If the structure of the molecule is known, $\mathrm{Rg}_{\mathrm{g}}$ can be estimated from the atomic coordinates:

$$
R_{g}^{2}=\frac{\sum_{i} A_{i}\left[\left(x_{i}-x_{c}\right)^{2}+\left(y_{i}-y_{c}\right)^{2}+\left(z_{i}-z_{c}\right)^{2}\right]}{\sum_{i} A_{i}}
$$

where $A_{i}$ is the atomic number of the atom $i, x_{c}, y_{c}$, and $z_{c}$ is the position of the electron center of the protein molecule.

\subsubsection{Molecular Weight (MW)}

Another direct structural parameter obtained from small angle $\mathrm{x}$-ray scattering is the molecular weight (MW) of the particle. $M W$ is directly related to the forward scattering intensity, $\mathrm{I}(0)$ as: $\mathrm{I}(0) \propto \mathrm{cM}_{\mathrm{r}}$, where $\mathrm{c}$ is the concentration in $\left(\mathrm{g} / \mathrm{cm}^{3}\right) . \mathrm{I}(0)$ is derived from the Guinier approximation of equation [6], or from the $P(r)$ function of equation [7]. To estimate the MW of the molecule, a sample with known MW is used as a standard for calibration:

$$
\frac{(\mathrm{I}(0) / \mathrm{c})_{\mathrm{c} \rightarrow 0, \text { molecule }}}{(\mathrm{I}(0) / \mathrm{c})_{\mathrm{c} \rightarrow 0, \text { standard }}}=\frac{\mathrm{MW}_{\text {molecule }}}{\mathrm{MW} \text { standard }}
$$

where $(\mathrm{I}(0) / \mathrm{c})_{\mathrm{c} \rightarrow 0}$ is the concentration-normalized forward scattering intensity at infinitely dilute solution. In our experiments, lysozyme is frequently used as a standard sample.

\subsubsection{Shape Information $-P(r)$ Function and $D_{\max }$}

The distance distribution $(\mathrm{P}(\mathrm{r})$ ) function can be calculated from the scattering curve using the direct or indirect Fourier transform methods. Since the $P(r)$ function represents the sample scattering in real space, the $P(r)$ function offers a more direct structural interpretation than the scattering intensity $\mathrm{I}(\mathrm{S})$. Molecules with distinct structural features, such as dumbbell-shaped or highly elongated, show particular patterns in the $P(r)$ function (Glatter \& Kratky, 1982). 
A molecular parameter $D_{\max }$ can be obtained directly from the $P(r)$ function. $D_{\max }$ is defined where $P(r)$ first reaches zero (Glatter \& Kratky, 1982). $D_{\max }$ describes the largest dimension of the molecule.

A further shape analysis involves an approximation of the molecule with simple geometrical models (Feigin \& Svergun, 1987; Glatter \& Kratky, 1982). A simple treatment is to model the whole molecule with a regular triaxial body, such as an ellipsoid, an elliptical cylinder or a prism. In Chapter 3 of this dissertation, rectangular prisms are used to represent the overall shapes of the ATPase and the peptide binding domains. A more complex approach is to model the molecule as a composite of regular triaxial bodies. This highly simplified modeling can, however, exclude some plausible models for the molecule (Wilbanks, et al., 1995).

\subsubsection{Kratky Plot}

The innermost part of the scattering curve, which contains information of the overall shape of the molecule, can be approximated as a Gaussian curve as in equation [6]. Due to the reciprocal relation, scattering at the large angular region is correlated to the structural features inside the molecule. Analysis of scattering intensity at the large angle region is complex, and depends on the structural detail of the molecule studied. However, some information can still be generalized.

It has been shown by Kratky (Glatter \& Kratky, 1982) that for a chain-like molecule scattering intensity at the large angle is inversely proportional to $S: I \propto S^{-1}$. Thus, in the IS ${ }^{2} v s$ S representation, or Kratky plot, a linear persistent increase is observed. On the other hand, for a globular-shaped molecule, scattering at the large angle follows Porod's law (Glatter \& Kratky, 1982), that is I $\propto \mathrm{S}^{-4}$, so that IS ${ }^{2}$ decreases rapidly with $S$ at large angle region. A well-defined peak in the Kratky plot is thus a characteristic of globular compact molecule. A coiled molecule and a globular molecule can accordingly be immediately distinguished in Kratky plots.

Kratky plots have been widely applied to the study of biological system, particularly in protein folding research (Kataoka, et al., 1993). Unfolded protein molecules can be treated as chain-like, while folded native molecules are compact and globular. Chapter 4 describes the application of Kratky plots in lysozyme folding studies. 


\subsection{General Experimental Aspects}

All the $x$-ray scattering experiments described in this dissertation were performed on beamline 4-2 at the Stanford Synchrotron Radiation Laboratory (SSRL). Some of the experimental aspects are summarized in the following. Relevant information regarding small-angle $x$-ray scattering experiments on this beamline can be found in the references (Eliezer, 1994; Rice \& Wakatsuki, 1991; Wakatsuki, et al., 1992).

Fig. 1.1 shows a schematic drawing of the small-angle $\mathrm{x}$-ray scattering experimental setup on beamline 4-2 at SSRL. A typical SAXS experiment setup includes: a (monochromatic) $\mathrm{x}$-ray source, a collimation system, a sample cell, a scattering path and a detector.

Synchrotron radiation. All the scattering data in this dissertation were taken using a synchrotron x-ray source. A detailed description of the basic physics of synchrotron radiation can be found in the literature (Landau \& Lifshitz, 1987; Winick \& Doniach, 1982). Briefly, synchrotron radiation is the electromagnetic field radiated by relativistic accelerated charged particles. The charged particles are either electrons or positrons, and they are accelerated using a magnetic field. To recirculate the particles along a circular path, magnetic fields perpendicular to the horizontal orbital plane are used. Radiation emitted from such relativistic particles is highly collimated in the direction tangential to the circular orbital. This is the qualitative explanation for the extreme brightness of a synchrotron radiation source, compared with a conventional radiation generated in a $\mathrm{x}$-ray tube. To further increase the brightness of radiation, wigglers and undulators are frequently used in synchrotron facilities.

Small divergence in the $\mathrm{x}$-ray beam is the primary concern in small-angle $\mathrm{x}$-ray scattering experiment. Although synchrotron radiation is highly collimated, the source is usually located far away ( $-26 \mathrm{~m}$ between the source and beamline $4-2$, for example), consequently, a few milliradian emittant angle results in a few centimeter divergence at the experimental station. A mirror is thus used to focus the $\mathrm{x}$-rays. Platinum $(\mathrm{Pt})$ is commonly chosen as the coating material for mirrors because of its long lifetime. A monochromatic beam is another factor in SAXS experiment. Monochromatic $x$-rays are selected by monochromators. In our experiments, an energy of about $8980 \mathrm{eV}$ is normally used, 

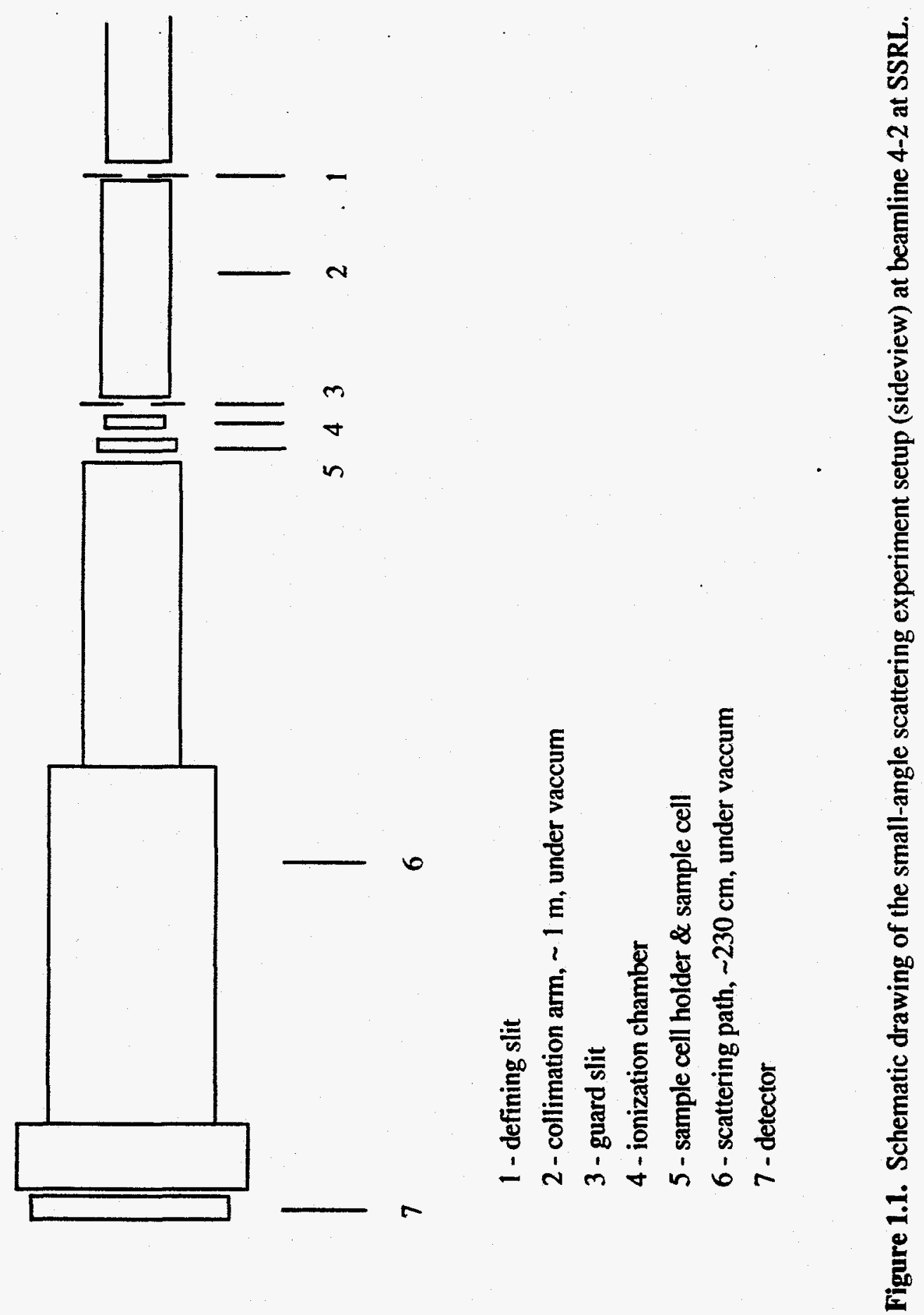
obtained by using a double-crystal monochromator setup with $\mathrm{Si}$ [111] crystals. A pair of $\mathrm{Mo} / \mathrm{B}_{4} \mathrm{C}$ multilayers is used for the experiments with the broad-band pass beam.

Sample considerations. Solution sample has to be contained in a cell. The window material for the cell can be mica, quartz, or glass with $10-50 \mu \mathrm{m}$ thickness. Two factors are considered in determining the optimum thickness of the sample. The scattering intensity increases linearly with the thickness ( $t$ ), while the absorption of $x$-rays by the sample increases exponentially, $\mathrm{e}^{-\mu \mathrm{t}}$, with $\mu$ being the linear absorption coefficient of the sample. For an approximation, the maximum scattering intensity is achieved when the function te ${ }^{-\mu t}$ is maximum, which occurs at $t=1 / \mu$. For dilute biological samples in water solution, absorption of the sample is mainly determined by the absorption of $\mathrm{H}_{2} \mathrm{O}$. For $\mathrm{H}_{2} \mathrm{O}, \mu=1 \mathrm{~mm}^{-1}$, thus the optimum thickness of the sample is about $1 \mathrm{~mm}$.

The other consideration about biological samples concerns radiation damage. Biological samples may degrade when exposed to the highly intense synchrotron $\mathrm{x}$-rays in the course of data collection. Changes in sample color or sample precipitation are the direct indications of radiation damage. Biological assay is normally carried out after the SAXS experiment if it is applicable to ensure that the sample is still biologically active. A further examination is to inspect the time course of scattering curves, particularly in the small angle region. There is no generalized method to prevent radiation damage to biological sample in a SAXS experiment, however, it has been found from our experience that addition of $\beta$ mercaptoethanol is effective in retarding protein sample deterioration in $\mathrm{x}$-rays.

Detectors. Two kinds of detectors have been used in the experiments described in this dissertation. A linear position proportional counter is routinely used in our SAXS experiments. It consists of two electrodes housed in a pressurized cell. The anode is a 6 $\mu \mathrm{m}$ gold tungsten coated wire, and the cathode consists of an array of metal coated glass fiber attached to the delay lines. A high electric field $(\sim 1.7 \mathrm{keV})$ is applied between the anode and the cathode. A detecting gas, consisting of either $\mathrm{Xe} / \mathrm{CO}_{2}$ or $\mathrm{Ar} / \mathrm{CO}_{2}$, acts as a conversion medium for $\mathrm{x}$-rays. The conversion efficiency of $\mathrm{Xe} / \mathrm{CO}_{2}$ is about 5 times that of $\mathrm{Ar} / \mathrm{CO}_{2}$, however, the cost of $\mathrm{Xe} / \mathrm{CO}_{2}$ is very high.

When a photon enters the detector chamber, it ionizes $\mathrm{Xe}$ or $\mathrm{Ar}$ in the conversion gas. Electrons thus generated are accelerated by the very high electric field to the anode wire. The energetic accelerated electrons in turn ionize other $\mathrm{Xe}$ or $\mathrm{Ar}$ atoms, and so on, triggering an avalanche and giving rise to a large amplification of the initial signal. When a 
signal arrives at the anode, it results in a charge buildup on the cathode. The electric pulses are then picked up by the delay lines and propagate in both directions, and finally reach the ends of the delay lines at different times. The time difference between the delay lines is correlated to the position of the ionization event. The $\mathrm{Xe}^{+}$or $\mathrm{Ar}^{+}$ions are neutralized by the quenching gas $\mathrm{CO}_{2}$. A quadrant detector, also used in some experiments, works in a similar fashion except that the detection area is larger and the delay signal detection is more sophisticated. A more detailed description of principles of both the linear and quadrant detectors can be found in Rice \& Wakatsuki, 1991.

\subsection{References}

Debye, P. (1915). Ann. Physik, 28, 809-823.

Eliezer, D. (1994) Protein folding and protein metallocluster studies using synchrotron small angle $x$-ray scattering. Ph.D. Thesis, Stanford University.

Fedorov, B. A., \& Denesyuk, A. I. (1978). FEBS Lett., 88, 114-117.

Feigin, L. A., \& Svergun, D. I. (1987). Structure analysis by small-angle $x$-ray and neutron scattering. New York: Plenum Press.

Glatter, O., \& Kratky, O. (Ed.). (1982). Small angle $x$-ray scattering. New York: Academic press.

Guiner, A., \& Fournet, G. (1955). Small-angle scattering of $x$-rays (C. B. Walker, Trans.). New York: John Wiley \& Sons, Inc.

Hubbard, S. R. (1987) Small-angle $x$-ray scattering studies of calcium-binding proteins in solution. Ph.D. Thesis, Stanford Univiersity.

Kataoka, M., Hagihara, Y., Mihara, K., \& Goto, Y. (1993). J. Mol. Biol., 229, 591596.

Landau, L., \& Lifshitz, E. M. (1987). In The classical theory of fields. Oxford: Pergamon.

Olah, G. A., Trakhanov, S., Trewhella, J., \& Ouiocho, F. A. (1993). J. Biol. Chem., 268, 16241-16247.

Rice, M., \& Wakatsuki, S. (1991). A user's guide to the small-angle x-ray scattering/diffraction data acquisition system at SSRL.

Semenyuk, A. V., \& Svergun, D. I. (1991). J. Appl. Crystallogr., 24, 537-540.

Svergun, D. I., Barberato, C., \& Koch, M. H. (1995). J. Appl. Crystallogr, submitted for publication.

Wakatsuki, S., Hodgson, K. O., Eliezer, D., Rice, M., Hubbard, S., Gillis, N., Doniach, S., \& Spann, U. (1992). Rev. Sci. Instrum., 63, 1736-1740. 
Wilbanks, S. M., Chen, L., Tsuruta, H., Hodgson, K. O., \& McKay, D. B. (1995). Biochemistry, 34, 12095-12106.

Winick, H., \& Doniach, S. (Ed.). (1982). Synchrotron radiation research. New York: Plenum. 


\section{Chapter 2}

Solution Small-angle X-Ray Scattering Study of the Components of the Azotobacter vinelandii Nitrogenase Enzyme System 


\subsection{Introduction and Background}

Nitrogenase is the biological enzyme system that reduces atmospheric $\mathrm{N}_{2}$ to ammonia, the main usable form of nitrogen for all organisms. The striking feature of this system is that the biological $\mathrm{N}_{2}$ fixation occurs under normal atmospheric pressure and at normal temperature, whereas the equivalent industrial Haber-Bosch process requires high pressure $(200-300 \mathrm{~atm})$, elevated temperature $\left(450^{\circ} \mathrm{C}\right)$, and catalysts (Nielsen, 1968). The mechanism of nitrogenase, therefore, has been under extensive investigation since the mid 1960s. A wealth of information gathered from biochemistry, genetics, inorganic chemistry, and particularly structural biology, has expanded our understanding of many aspects of this biological $\mathrm{N}_{2}$ fixation system.

One of the most intensively investigated nitrogenase systems is the molybdenumcontaining nitrogenase from Azotobacter vinelandii, a free-living aerobic bacterium. The nitrogenase enzymatic system consists of two $\mathrm{O}_{2}$-sensitive metalloproteins: the $60 \mathrm{kDa}$ iron protein (Fe protein) and the $230 \mathrm{kDa}$ molybdenum-iron protein (MoFe protein). Schematic representations of these two components are shown in Fig. 2.1. In the process of $\mathrm{N}_{2}$ fixation, the Fe protein transfers one electron from an in vivo donor such as ferredoxin or flavodoxin to the MoFe protein. Then the MoFe protein utilizes electrons to reduce $\mathrm{N}_{2}$ to ammonia (Burgess, 1983; Thorneley \& Lowe, 1985). Hydrolysis of two MgATP molecules is required for one electron transfer from the Fe protein to the MoFe protein to occur. The overall stoichiometry of $\mathrm{N}_{2}$ reduction by nitrogenase has been established as (Burgess, 1983):

$$
\mathrm{N}_{2}+8 \mathrm{H}^{+}+8 \mathrm{e}^{-}+16 \mathrm{MgATP} \rightarrow 2 \mathrm{NH}_{3}+\mathrm{H}_{2}+16 \mathrm{MgADP}+16 \mathrm{Pi}_{\mathrm{i}}
$$

In addition to $\mathrm{N}_{2}$, nitrogenase also supports reduction of $\mathrm{C}_{2} \mathrm{H}_{2}$ to $\mathrm{C}_{2} \mathrm{H}_{4}, \mathrm{~N}_{3}^{-}$to $\mathrm{N}_{2}$ $+\mathrm{NH}_{3}$, and $\mathrm{RCN}$ to $\mathrm{RCH}_{3}+\mathrm{NH}_{3}$.

\subsubsection{The Fe Protein}

The Fe protein is a dimer of two identical subunits, which are covalently connected by a $[4 \mathrm{Fe}-4 \mathrm{~S}]^{2+11+}$ cluster at one end of the dimer. There are two nucleotide binding sites, one in each subunit (Burgess, 1993; Yates, 1991). Sequence comparisons (Robson, 1984), site-directed mutagenesis (Seefeldt, et al., 1992) and x-ray crystallography (Georgiadis, et al., 1992) are all consistent with a model whereby the terminal phosphate of 

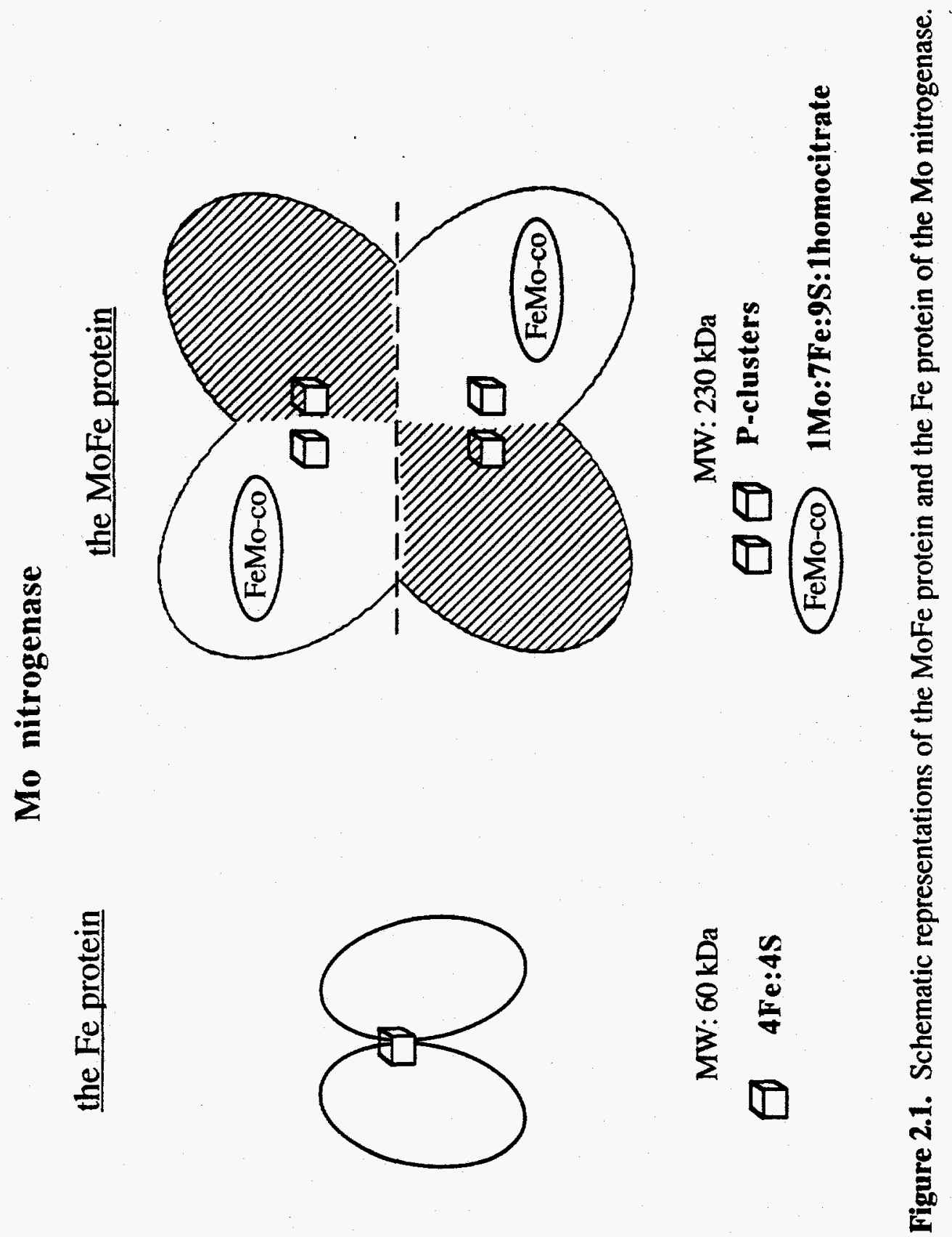
MgATP interacts with the "Walker's motif" sequence GXGXXGKS (Walker, et al., 1982) which is located between residues 9-16 of the Azotobacter vinelandii Fe protein sequence. One of the most iniriguing aspects of the Fe protein is that MgATP binding somehow influences the environment of the [4Fe-4S] cluster (Burgess, 1983; Burgess, 1993; Yates, 1991), even though the binding site for the terminal phosphate is located $20 \AA$ away (Georgiadis, et al., 1992). Changes in the [4Fe-4S] cluster that occur upon MgATP binding include: 1) the reduction potential $\mathrm{E}^{0}$ is lowered by $c a .100 \mathrm{mV}$ (Morgan, et al., 1986; Zumft, et al., 1974); 2) the S=1/2 EPR signal exhibited by [4Fe-4S] ${ }^{1+}$ changes from a rhombic shape to a more axial shape (Orme-Johnson, et al., 1972; Smith, et al., 1973; Zumft, et al., 1972);3) the $\mathrm{O}_{2}$ sensitivity increases; 4) large changes occur in the $\mathrm{CD}$ spectrum of the oxidized protein (Stephens, et al., 1982); and 5) the cluster Fe atoms, which are inert to chelators in the absence of MgATP, become accessible to chelators in its presence (Walker \& Mortenson, 1973; Walker \& Mortenson, 1974). Thus, MgATP binding appears to induce a long-distance conformational change in the Fe protein. Mutagenesis data further suggest that this conformational change is a prerequisite for the next step in nitrogenase turnover, which is the binding of the Fe protein to the MoFe protein in such a way that electron transfer can occur (Gavini \& Burgess, 1992; Seefeldt, et al., 1992).

It is interesting that the effects of MgATP and MgADP are not identical. Binding of $\mathrm{MgADP}$ to the $\mathrm{Fe}$ protein causes changes in the $\mathrm{CD}$ spectrum and reduction potential $\mathrm{E}^{0^{\circ}}$ (Burgess, 1983; Morgan, et al., 1986; Stephens, et al., 1982; Zumft, et al., 1974); but it is an inhibitor of the chelator reaction (Walker \& Mortenson, 1973; Walker \& Mortenson, 1974). Thus, even though the two nucleotides appear to bind to the same sites (Tso \& Burris, 1973) they have different effects on protein structure. These changes in turn are important for function because MgATP is required for substrate reduction while MgADP inhibits substrate reduction.

A mutant variant of the $A$. vinelandii Fe protein, designated $A$. vinelandii UW91 (Shah, et al., 1973), which has a serine substituted for an alanine at position 157 of the NifH sequence (Gavini \& Burgess, 1992), has been purified and characterized. This mutant has a normal $[4 \mathrm{Fe}-4 \mathrm{~S}]^{2+/ 1+}$ cluster, and normal MgATP binding ability but it is blocked for the MgATP-induced conformational change as measured by the chelation reaction described above (Gavini \& Burgess, 1992). This altered Fe protein also cannot support substrate reduction (Gavini \& Burgess, 1992). 


\subsubsection{The MoFe Protein}

The MoFe protein is a heterotetramer $\alpha_{2} \beta_{2}$, with two types of metal clusters: two iron-molybdenum (FeMo) cofactors, and four [4Fe-4S] clusters (P-clusters) (Burgess, 1983). The FeMo cofactor is believed to be the substrate, $\mathbf{N}_{2}$, binding and reducing site (Burgess, 1990). The P-cluster is proposed to bridge the electron transfer between the [4Fe-4S] cluster of the Fe protein and the FeMo cofactor of the MoFe protein (Holm, 1990). The MoFe protein has two Fe protein binding sites, and interacts only transiently with the Fe protein (Thomeley \& Lowe, 1985). Although the Fe protein can bind MgATP by itself, the hydrolysis of ATP in the electron transfer from the Fe protein to the MoFe protein, only occurs within the Fe protein/MoFe protein complex. To ensure that electron transfer occurs in this binary protein complex, a correct orientation of the proteins to align the electron donor and acceptor sites must be maintained. So far there is no crystal structure available for this active complex. Several models, however, have been proposed based on cross-linking and mutagenesis studies (Thorneley, et al., 1993; Willing \& Howard, 1990).

\subsubsection{The FeMo Cofactor}

The FeMo cofactor was first isolated in 1977 as a stable metallocluster from aciddenatured MoFe protein (Shah \& Brill, 1977). It is extremely sensitive to oxygen; yet it is stable in $N$-methylformamide (NMF) at room temperature under anaerobic conditions. The isolated FeMo cofactor can be inserted back into cofactor-deficient MoFe protein, upon which the activity of the MoFe protein is restored. Since the cofactor is believed to be the active site for $\mathrm{N}_{2}$ reduction, extensive research has been devoted to study the structure and function of this metal cluster, including great efforts in chemical modeling aspects. However, the isolated FeMo-cofactor has not yet been crystallized successfully. A major breakthrough came with the publication of the structure of cofactor inside the MoFe protein (Kim \& Rees, 1992), shown in Fig. 2.2. It contains two subclusters: the $\mathrm{MoFe}_{3} \mathrm{~S}_{3}$ and the $\mathrm{Fe}_{4} \mathrm{~S}_{3}$ subclusters with a total of $1 \mathrm{Mo}: 7 \mathrm{Fe}: 9 \mathrm{~S}$, in addition to two protein ligands and a homocitrate. Structures of the cofactor in solution and inside the protein appear to be similar as determined by extended x-ray absorption fine structure (EXAFS), Mössbauer, and electron paramagnetic resonance (EPR) spectroscopjes (Burgess, 1990; Conradson, et al., 1987; Hedman, et al., 1988; Rawlings; et al., 1978). 

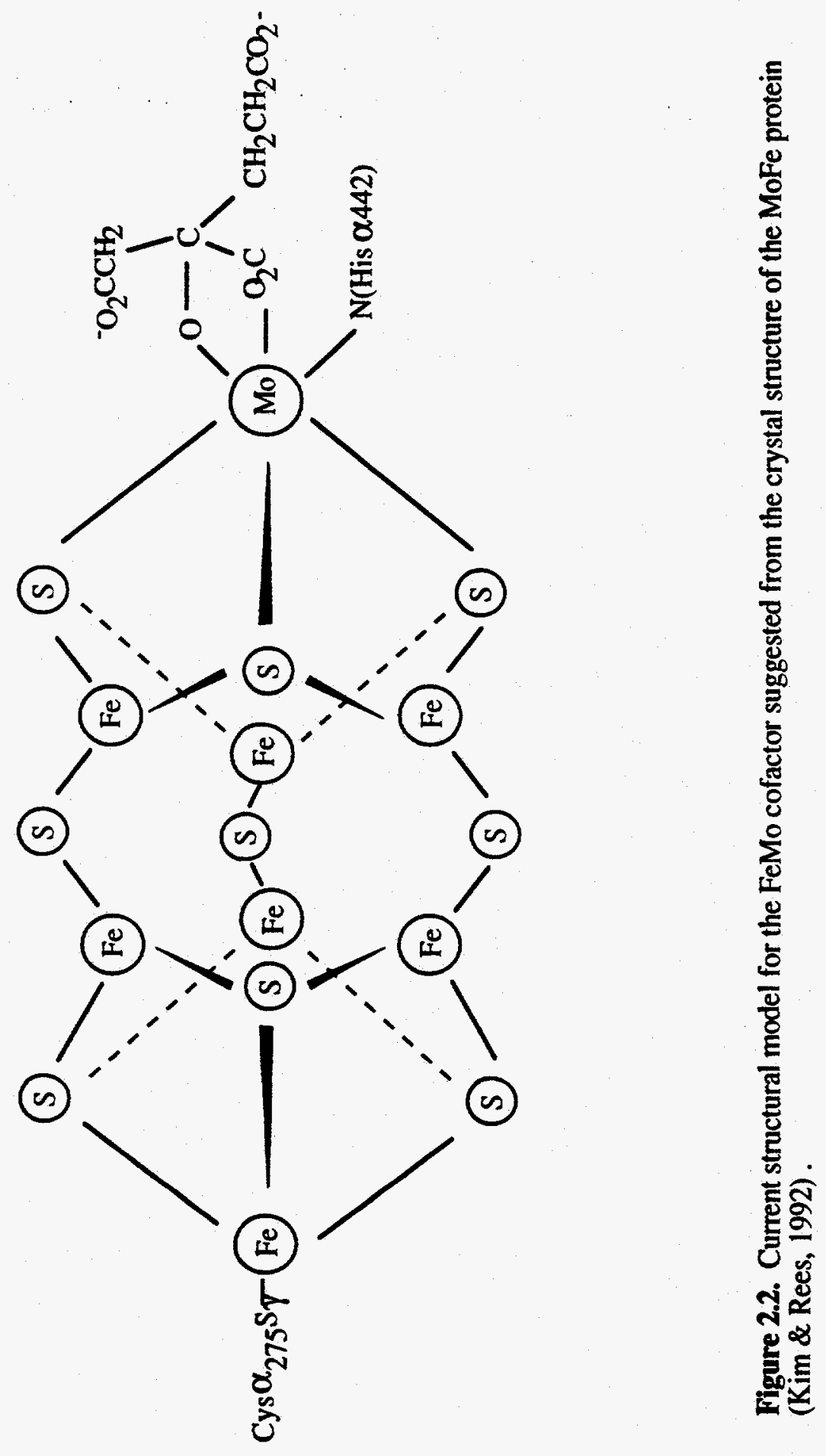


\subsubsection{The FeMo Cluster}

A Mo-containing metal cluster (designated as FeMo cluster) has been isolated from the MoFe protein by a preparation method (Shah \& Brill, 1981) different from that for the FeMo-cofactor: Instead of extraction into $N$-methylformamide (NMF) under alkaline condition ( $\mathrm{pH} \sim 8.5$ ), the extraction of the FeMo cluster is carried out at acidic $\mathrm{pH}(\mathrm{pH} \sim 4)$ in methyl-ethyl ketone (MEK). This entity exhibits a very similar $\mathrm{S}=3 / 2$ EPR signal, a spectroscopic feature whose magnetic center is still not clear, to those of the isolated FeMo cofactor and the FeMo cofactor within the protein. The fact that the cluster inhibits the insertion of the cofactor back into cofactor-deficient MoFe protein indicates that the cluster binds to the same site as the cofactor (or nearby) in the MoFe protein (Burgess, 1990). However, they differ structurally. Circular dichroism indicates that the cluster does not contain homocitrate; both $\mathrm{Fe}$ analysis and EXAFS show that the Mo atom of the cluster has one less $\mathrm{Fe}$ atom in the first coordination shell and EXAFS in addition indicates a change in the long range order (Ma, et al., 1994). Furthermore, this cluster can not activate the FeMo cofactor-deficient form of the MoFe protein.

It has been shown by small-angle $\mathrm{x}$-ray scattering that FeMo cofactor in NMF solution is not homogenous (Eliezer, et al., 1993), and this inhomogeniety may account for the failure in crystallizing the cofactor for structural determination. The cofactor exists in large aggregates (up to $25 \AA$ in $R_{g}$ ) as well as in small oligomeric forms. The heterogeneity of the cofactor was suggested to be due to the presence of different ligation states of the endogenous homocitrate (Ma, et al., 1994). Since homocitrate is not retained in the FeMo cluster, the comparison between the aggregation states of the cofactor and the cluster would provide immediate direct information on the effect of homocitrate on oligomerization. Efforts have been devoted to search for conditions to crystallize the cofactor outside the protein matrix for structural determination, and a homogenous, aggregate-free system is crucial for producing diffraction-quality crystals.

In this chapter, emphasis is placed on $\mathrm{x}$-ray scattering studies of the Fe protein, which undergoes a conformational change triggered by MgATP. Some initial results of scattering studies of the MoFe protein as well as the FeMo cluster are also presented. 


\subsection{Experimental Procedures}

\subsubsection{The Fe Protein}

The wild type and the A. vinelandii UW91 Fe proteins were purified as described elsewhere (Burgess, et al., 1980; Gavini \& Burgess, 1992). They were homogeneous by the criteria of one- and two-dimensional SDS-PAGE (sodium dodecylsulfatepolyacrylamide gel electrophoresis). The native protein had a specific activity of $c a .1,900$ nmoles of $\mathrm{H}_{2}$ evolved/min/mg protein and had no background activity. A complete characterization of the UW91 Fe protein, which is inactive, has been described (Gavini \& Burgess, 1992).

All Fe protein samples were prepared anaerobically in a Vacuum Atmospheres glove box under dry nitrogen atmosphere. Stock Fe protein solutions $(30 \mathrm{mg} / \mathrm{mL}$ in 0.05 $\mathrm{M}$ Tris- $\mathrm{HCl}, 0.5 \mathrm{M} \mathrm{NaCl}, 2 \mathrm{mM} \mathrm{Na}_{2} \mathrm{~S}_{2} \mathrm{O}_{4}, \mathrm{pH} 8.0$ ) were first diluted to the appropriate concentrations with the same buffer. Where MgATP was needed, the solution was then made $2.5 \mathrm{mM}$ in MgATP using a $25 \mathrm{mM}$ stock solution. All samples were then adjusted to $5 \mathrm{mM}$ in sodium dithionite by addition of the appropriate amount of a $300 \mathrm{mM} \mathrm{pH}$-adjusted $\mathrm{Na}_{2} \mathrm{~S}_{2} \mathrm{O}_{4}$ stock solution. Both sample preparations and sample integrity tests (before/after SAXS experiments) were carried out by Dr. Narasaiah Gavini in Prof. Burgess' laboratory at UC-Irvine.

For the 1993 runs (see below), $5 \mu \mathrm{l}$ of a $200 \mathrm{mM}$ pH-adjusted $\mathrm{Na}_{2} \mathrm{~S}_{2} \mathrm{O}_{4}$ solution were added into each $495 \mu \mathrm{l}$ protein sample solution just before the sample was loaded for scattering measurement. Based on the previous SAXS experience with the Fe protein, this was done to further ensure that the proteins were in a reducing environment. Identical samples, that were stored frozen at UC-Irvine for several weeks, were subsequently assayed for $\mathrm{C}_{2} \mathrm{H}_{2}$ reduction activity and were tested for the MgATP-induced conformation change by adding bathophenanthroline disulfonate (Ljones \& Burris, 1978). All the wildtype samples showed $\mathrm{C}_{2} \mathrm{H}_{2}$ reduction activity within experimental error of the starting values. As previously reported (Gavini \& Burgess, 1992), all native samples containing MgATP released their Fe atoms when the chelator was added, whereas the native samples without MgATP and all UW91 samples, did not. 
Scattering experiments were performed on beamline 4-2 at Stanford Synchrotron Radiation Laboratory (SSRL) in May 1992, January and May 1993 using the SSRL smallangle $\mathrm{x}$-ray scattering/diffraction system (Wakatsuki, et al., 1992). Synchrotron radiation was provided by the storage ring SPEAR, which was operated at an electron energy of 3.0 $\mathrm{GeV}$ with a ring current of 40-90 $\mathrm{mA}$. The synchrotron beam was monochromatized (to an energy of $8980 \mathrm{eV}$ ) by a double-crystal $\mathrm{Si}[111]$ monochromator. The typical beam size at the sample position was $1.0 \mathrm{~mm}(\mathrm{v}) \times 2.1 \mathrm{~mm}$ (h) for a flat-window cell in the 1993 runs, and $0.4 \mathrm{~mm}(\mathrm{v}) \times 3.0 \mathrm{~mm}(\mathrm{~h})$ for capillary cells in the $1992 \mathrm{run}$. Scattering data were collected with a one-dimensional position-sensitive proportional counter (BioLogic, Grenoble, France) with a $200 \mathrm{~mm}$ long sensitive area. A short-path, open-air ionization chamber was placed immediately in front of the sample to record the incident $\mathrm{x}$-ray intensity on the sample. The total flux on the sample was used to normalize scattering intensity against small fluctuations and intensity changes of the $x$-ray beam due to the decay of the electron current in SPEAR. The sample-to-detector distance was determined to be 264 ( \pm 1) $\mathrm{cm}, 265( \pm 1) \mathrm{cm}, 230( \pm 1) \mathrm{cm}$ for the May 1992, January and May 1993 runs, respectively.

Data were collected in a protein concentration series from $2 \mathrm{mg} / \mathrm{ml}$ to $12 \mathrm{mg} / \mathrm{ml}$ of five protein samples, wild-type without nucleotides, wild-type plus MgATP, wild-type plus MgADP, mutant UW91 without nucleotides and mutant UW91 plus MgATP. In the 1993 experiments, all data collection was performed using flat-window cells. These cells are made of Kel-F and have $25 \mu \mathrm{m}$ thick, $5 \mathrm{~mm}$ diameter circular mica windows on each side, with a $1.2 \mathrm{~mm}$ light path. Two small holes from the top into the cell body allow for sample injection. The holes are anaerobically sealed by a threaded acrylic plug with two $\mathrm{O}$ rings. The air tightness of the cells was satisfactory, as tested by the lack of change of the absorption spectra of a methyl viologen radical cation solution over a time longer than a typical SAXS measurement. During data collection, the cell was fit into a fixed cell holder with $6.3 \mu \mathrm{m}$ thick polypropylene film on each side. Helium was continuously flowed through the holder to eliminate air scattering around the sample, to provide a microanaerobic environment around the sample cell, and to keep the window surface clean. The temperature was maintained at $20^{\circ} \mathrm{C}$ by circulating an ethylene glycol solution through the sample holder. Three types of buffer solutions (without nucleotides, with $2.5 \mathrm{mM}$ MgATP, and with $2.5 \mathrm{mM} \mathrm{MgADP)}$ were used as background solutions for the corresponding protein samples. Background scattering was recorded for every two protein samples. The exposure time for each buffer and protein solution was the same, $8 \times 5$ minutes. 
For the 1992 run when capillaries were employed, the following procedure was used for every set of data collection. Background scattering from a $1.5 \mathrm{~mm}$ diameter $\mathrm{x}$-ray glass capillary (Charles Supper Company) filled with the buffer solution was first recorded, and the buffer was subsequently removed. Then a ca. $80 \mu \mathrm{l}$ aliquot of a protein solution was loaded, the capillary end was sealed with 5-minute epoxy glue (Devcon Corporation), and then data were collected. Every effort was taken to ensure that the capillary position was invariant with respect to the beam. The exposure time for each buffer and protein was $3 \times 10$ minutes. This capillary method was used initially until the reliable, anaerobic flat-window cells were developed and tested. Higher parasitic scattering from the round capillary wall and the resultant poor background subtraction make the capillary measurements less reproducible. For both the flat-window cell and the capillary experiments, all sample handling involving the proteins was carried out anaerobically in a Vacuum-Atmospheres dry $\mathrm{N}_{2}$-filled glove box.

\subsubsection{The MoFe Protein}

The MoFe protein was obtained anaerobically during the purification of the wild type Fe protein (Burgess, et al., 1980). It was purified to homogeneity as monitored by one- and two-dimensional SDS-PAGE. The specific activity of the MoFe protein was $3000 \mathrm{nmol}$ of $\mathrm{H}_{2}$ evolved $/ \mathrm{min} / \mathrm{mg}$ protein. Samples for SAXS experiments were prepared by dilution from a stock MoFe protein solution $(-40 \mathrm{mg} / \mathrm{ml}$ in $25 \mathrm{mM}$ Tris- $\mathrm{HCl}, 250 \mathrm{mM}$, $\mathrm{NaCl}, 2 \mathrm{mM}, \mathrm{Na}_{2} \mathrm{~S}_{2} \mathrm{O}_{4}, \mathrm{pH} 7.4$ ) with the same buffer to final concentrations of 2,4 , and 8 . $\mathrm{mg} / \mathrm{ml}$, and were frozen in dry ice until the SAXS experiment. Representative samples were recovered anaerobically after data collection for activity assay, and their activities were found close to the initial value. All the sample preparations were performed by Dr. Narasaiah Gavini in Prof. Burgess' laboratory at UC - Irvine.

SAXS data were collected in May 93 on beamline 4-2 at SSRL. A flat-window sample cell was used for data collection. All sample manipulation procedures, as well as the beamline configurations, were similar to those of the $\mathrm{Fe}$ protein experiments unless otherwise stated.. The sample-to-detector distance was $230( \pm 1) \mathrm{cm}$, which covered the angular range up to $0.04 \AA^{-1}$ in $S(S=2 \sin \theta / \lambda)$. Background was recorded immediately before or after the sample measurement, with data collection time being $8 \times 5$ minutes. 


\subsubsection{The FeMo Cluster}

The purification of the FeMo cluster was done by Dr. Li Ma in Prof. Burgess' laboratory at UC - Irvine according to reference (Ma, et al., 1994). Briefly, inside the dry box, the MoFe protein (in $25 \mathrm{mM}$ TrisHCl, $250 \mathrm{mM} \mathrm{NaCl}, 2 \mathrm{mM} \mathrm{Na}_{2} \mathrm{~S}_{2} \mathrm{O}_{4}, \mathrm{pH}$ 7.4) in a tube was placed underneath a layer of methyl ethyl ketone (MEK) which was presaturated with the same buffer. The tube, sealed with a serum stopper, was then removed from the dry box. Solution of $4 \mathrm{~N} \mathrm{HCl}$ was added into the MEK layer, and the tube was subjected to centrifugation at $4^{\circ} \mathrm{C}$. The cluster which was extracted into the top MEK layer was removed inside the dry box. To transfer the cluster into the NMF solution, the MEK solution was diluted with NMF anaerobically, and MEK was distilled away under vacuum. The NMF solution contained $20 \mathrm{mM}$ benzylthiol as the reducing agent, while the MEK solution contained saturated $\mathrm{Na}_{2} \mathrm{~S}_{2} \mathrm{O}_{4}$. The cluster samples were frozen at $-70^{\circ} \mathrm{C}$ in a crimp-top v-shape vial with a silicon septum covered with several layers of Saran Wrap, and shipped in dry ice to Stanford University.

The stock cluster solution was concentrated when necessary and then diluted into different concentrations with the appropriate buffer, either MEK or NMF. In the first experiment of June 94, the cluster was prepared in both MEK and NMF. The stock MEK solution (containing $\sim 1 \mathrm{mM}$ FeMo cluster) was diluted to lower concentrations, making a total of three samples: $\sim 1 \mathrm{mM}, \sim 0.8 \mathrm{mM}$ and $\sim 0.6 \mathrm{mM}$. The stock NMF solution was first concentrated at $20-30^{\circ} \mathrm{C}$ and 140 microns pressure, and then diluted to make three samples, $731 \mu \mathrm{M}, 619 \mu \mathrm{M}$ and $464 \mu \mathrm{M}$. In the June 1995 run, scattering data were collected only on the NMF solution because it was shown from the first run that the cluster predominantly formed large aggregates in the MEK solution. The concentration of the stock solution was $463( \pm 30) \mu \mathrm{M}$. A portion of this solution was retained as a scattering sample directly; the remainder was concentrated to dryness and redissolved in NMF to make a final concentration of $1.44( \pm 0.12) \mathrm{mM}$. Except for the MEK samples, the cluster concentrations were determined by iron analyses. All these sample manipulations were performed by Dr. Patrick Frank at Keith Hodgson's laboratory at Stanford University.

Since both MEK and NMF are organic solvents, they can dissolve the epoxy glue which adheres the mica windows to the Kel-F body of the flat-window cell. A special cell was therefore designed and constructed. The cell is built in the middle of a $40 \times 40 \times 20$ $\mathrm{mm}$ nylon block. The cell is $5 \mathrm{~mm}$ in diameter and $1.0 \mathrm{~mm}$ thick with a total volume of 
$30 \mu \mathrm{l}$. Instead of gluing windows to the cell, circular precut $25 \mu \mathrm{m}$-thick $10 \mathrm{~mm}$ in diameter mica (Attwater \& Sons Ltd., U.K.) sheets are attached to the cell surface via 0.1 mm-thick Teflon o-rings. The window is pressed firmly against the wall with a rubber gasket by a threaded nylon plug on each side. A hole of $6 \mathrm{~mm}$ in diameter is drilled through the plug in the middle, and coincides with the cell window. Like the flat-window cell, there are two tiny holes ( $1 \mathrm{~mm}$ in diameter) on the top for sample loading. The holes are sealed by a $2.25 \mathrm{~mm}$-thick circular silicone septum and a threaded plug. The cell has a stem on the bottom of the block which fits into the regular cell holder.

Anaerobicity of the cell was tested by monitoring the time course of any change in the iron absorption spectrum of $\left(\mathrm{Et}_{4} \mathrm{~N}\right)_{2}\left[\mathrm{Fe}^{\mathrm{II}}\left(\mathrm{S}_{2}-0-\mathrm{xyl}\right)_{2}\right]$ compound in $N, N$ dimethylformamide (DMF) solution. The spectrum was stable for at least $75 \mathrm{~min}$, and appeared to change slightly over the rest of the measuring time, which was about 4 hours. An approximate 1-electron oxidation rate for the FeMo cofactor was derived to be about 96 $\mathrm{nM} / \mathrm{min}$ by analogy with the $\mathrm{Fe}$ (II) model compound. Accordingly, even in the worst situation where data collection time was 5 hours and the sample was in the least concentration of $464 \mu \mathrm{M}$, only $6 \%$ of the cluster sample would be oxidized. The airtightness is therefore satisfactory for our cluster scattering experiment. The cell design and anaerobicity test were done by Dr. Patrick Frank at Prof. Keith Hodgson's laboratory at Stanford University.

Since the cluster is such a small molecule, $\sim 800 \mathrm{Da}$, much smaller than the usual small single domain proteins which are normally around $12 \mathrm{kDa}$ in molecular weight, much longer data collection time is expected. In practice, particularly for the first sample, data were collected on one hour base. At the end of each hour, the sample was inspected by eye, and if there was no visible anomaly such as color change or precipitate, data collection was resumed. Then continuous data collections were set at the longest time available, $4-5$ hours. Background scattering was recorded either before or after the sample. Samples were recovered anaerobically for EPR inspection.

\subsection{Data Analysis}

Data were analyzed using the Guinier approach, as described in Chapter 1 . Because the molecular weights of the scatterers vary from $220,000 \mathrm{Da}$ to $60,000 \mathrm{Da}$ to 800 $\mathrm{Da}$, and consequently a great difference in their sizes or $\mathrm{R}_{\mathrm{g}}$ is expected, the fitting range for 
the Guinier approximation was chosen accordingly: $3.2 \times 10^{-3}-6.3 \times 10^{-3}$ for the MoFe protein, $4.5 \times 10^{-3}-9.5 \times 10^{-3} \AA^{-1}$ for the Fe protein in S. For the FeMo cluster, $S_{\min }$ was selected where the parasitic scattering appears to be minimum, and $S_{\max }$ was chosen depending on the availability of data on higher angular range.

Radius of gyration can be calculated directly from the atomic coordinates:

$$
R_{g}^{2}=\frac{\sum_{i} A_{j}\left[\left(x_{j}-x_{c}\right)^{2}+\left(y_{i}-y_{c}\right)^{2}+\left(z_{i}-z_{c}\right)^{2}\right]}{\sum_{i} A_{i}}
$$

where $A_{i}$ is the atomic number of atom $i, x_{C}, y_{C}$, and $z_{c}$ are the coordinates of the electron density center of the protein molecule.

For protein molecules, scattering curves can also be calculated from atomic coordinates, including a $3 \AA$ thick hydration layer of water around the protein molecule. A value of $0.03 \mathrm{e}^{-1} / \AA^{3}$ is assumed to be the electron density contrast between the hydration layer and the bulk background. The calculations were performed using the program CRYSOL17 (Svergun, et al., 1995).

Although the detector response homogeneity was constantly checked during the experiment, in the data reduction, detector response correction was undertaken only on the most recent data, the FeMo cluster data. Before the 1994 run, a ${ }^{55} \mathrm{Fe}$ source was used to irradiate the detector, and as a result the response pattern was deformed somehow because of the point-like radiation source. Under these circumstances, the response pattern was taken only to ensure that no anomaly occurred to the detector particularly in the small angle regions during data collection. When an aberration was detected, the detector was cleaned until a reasonable response was obtained. In the recent runs, a more homogenous source of irradiation, fluorescence generated by direct $\mathbf{x}$-ray beam hitting a copper plate, was utilized instead. The detector response homogeneity was reflected more precisely with this method, and the response pattern was used to correct any subtle inhomogeneity of the detector by a channel-by-channel division of the data by the detector response. 


\subsection{Results and Discussion}

\subsubsection{The Fe Protein}

None of the samples measured showed a color change during/after the measurement which would have indicated sample oxidation/degradation. To further assure that no detectable radiation damage or aggregation occurred during the course of each experiment, an examination of normalized scattering curves from different cycles of data collection was carried out, particularly at the smallest angle region available. They overlapped within experimental error, suggesting that protein molecules remained in the same aggregation state throughout data collection process. A typical Guinier plot is shown in Fig. 2.3. The lack of upward curvature in the Guinier region indicates that no significant aggregation had taken place.

The apparent radii of gyration for each sample were derived from the Guinier approximation. The effect of concentration on radius of gyration was minimized as usual by the linear extrapolation of $\mathrm{R}_{\mathrm{g}}$ vs. concentration (C) to zero protein concentration. The extrapolations are shown in Fig. 2.4.a-e. The nearly linear dependence of the apparent $R_{g}$ on the protein concentration further demonstrates that the predominant aggregation state for each sample remained the same throughout the concentration range chosen. The forward intensity at zero scattering angle, $I(0)$, which can be obtained from the extrapolation of Guinier plots to zero scattering angle, is roughly proportional to the square of the mass of the scatterer (Glatter, 1982). The ratio (I(0)/C) at zero concentration obtained for all five protein samples were similar. This result was expected because of the small mass of MgATP and MgADP compared to that of the protein molecule. Plots of $(\mathrm{I}(0) / \mathrm{C})$ vs. concentration for all samples were acceptably linear which is again consistent with no change of protein aggregation state within the concentration range tested. Again it is important to note that the lack of change in aggregation state described above was observed and reproduced over three independent experimental runs.

Independent tests of reproducibility. Despite the use of different sample cells, the results of the capillary method adopted in the May 1992 run were reproduced in the January 1993 data using the flat-window cell method. To further examine the reproducibility of data, a number of multiple measurements were carried out under the most favorable and reproducible experimental conditions (with the flat-window cells, and an additional amount 

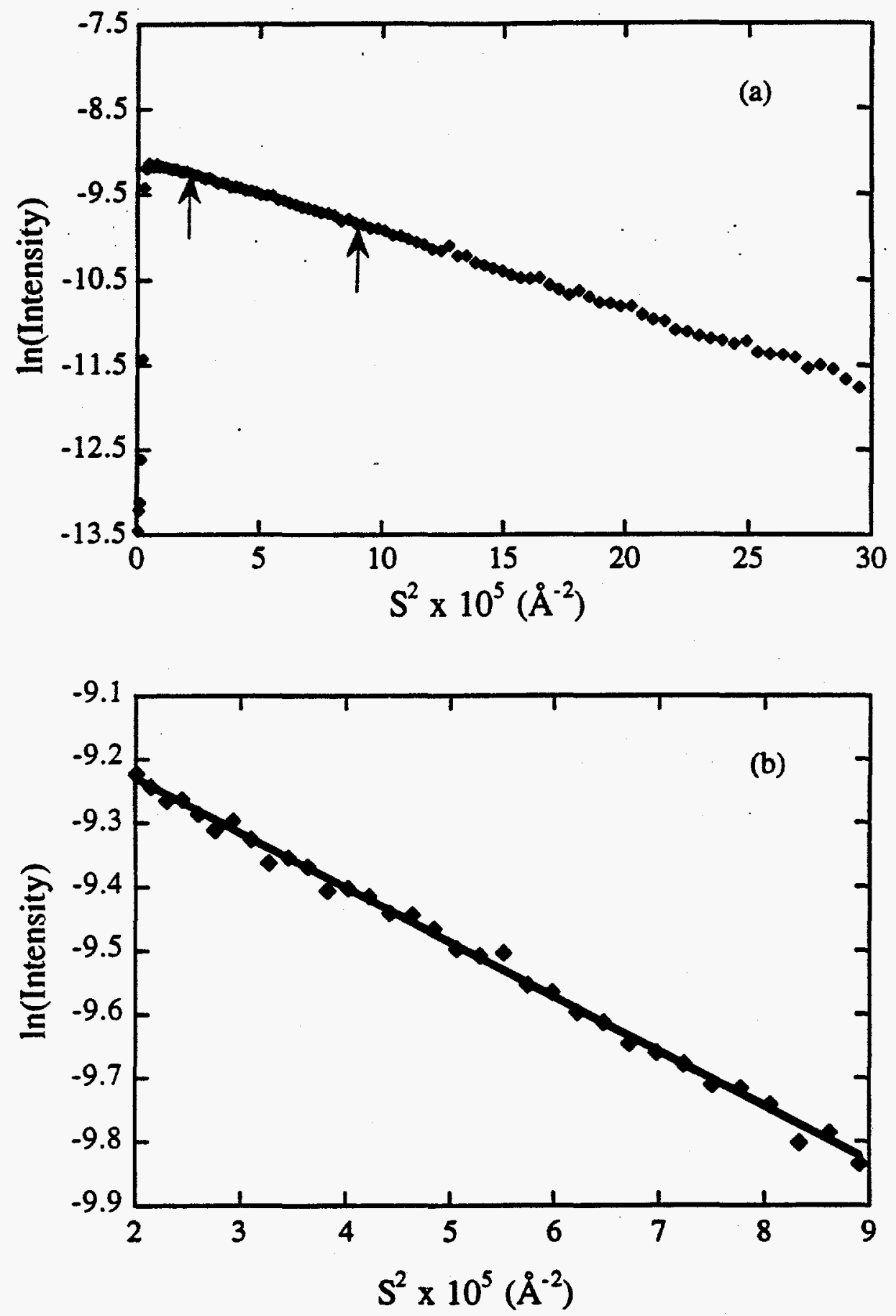

Figure 2.3. (a) A typical Guinier plot and region selected for Guinier fit (between arrows). (b) The expanded fitting region. Data were linearly fit by least squares methods. This data set is from the January 1993 run for wild-type Fe protein in the presence of MgATP at $12 \mathrm{mg} / \mathrm{ml}$. 

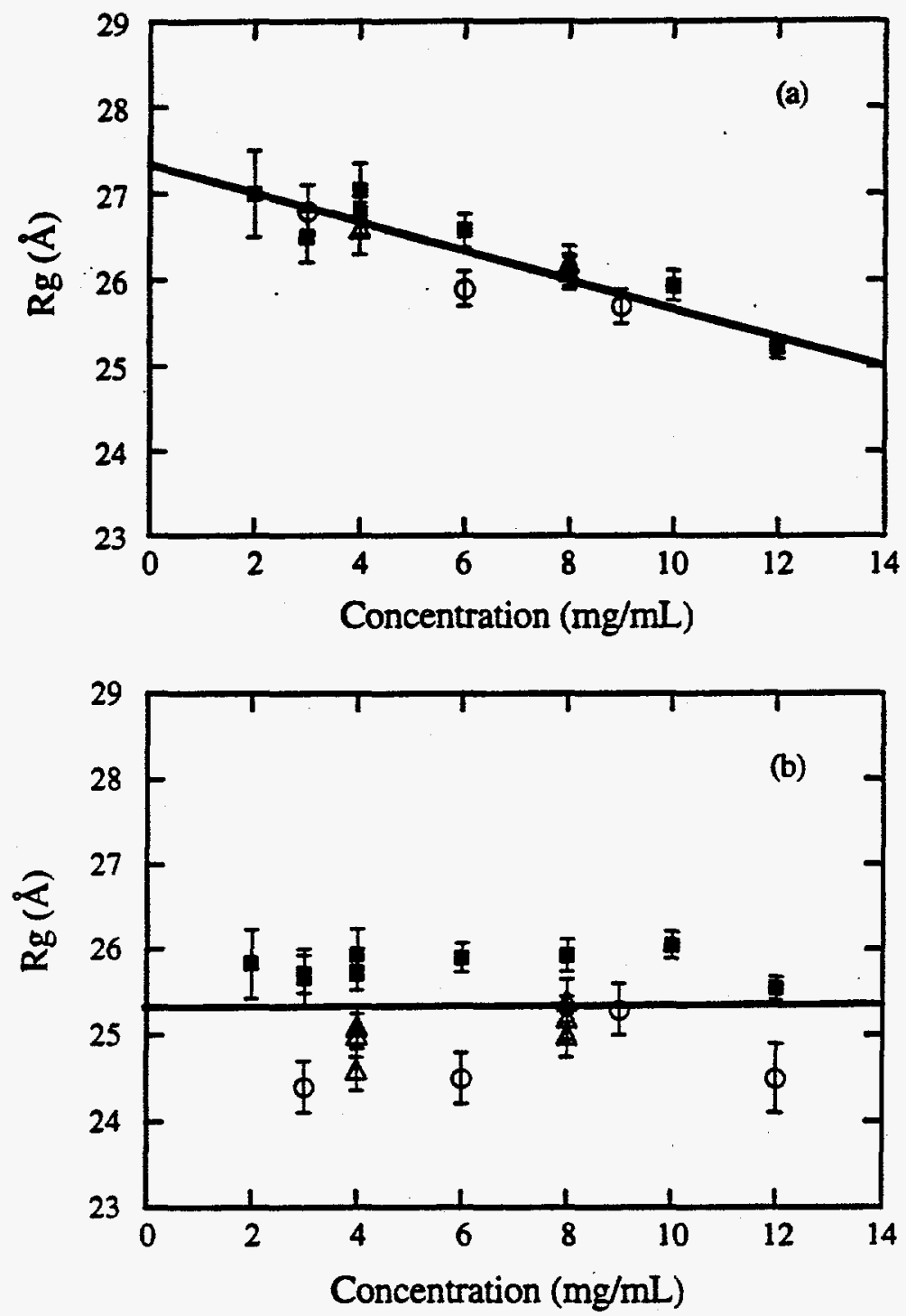

Figure 2.4. Radii of gyration as a function of (a) the wild-type Fe protein in the absence of nucleotides; (b) the wild-type Fe protein in the presence of MgATP; (c) the wild-type Fe protein in the presence of MgADP; (d) the mutant UW91 Fe protein in the absence of nucleotides; (e) the mutant UW91 Fe protein in the presence of MgATP. O, data of May $1992 \mathrm{run} ; \mathrm{D}$, data of January $1993 \mathrm{run} ; \Delta$, data of May 1993. Least squares methods were employed in the extrapolation. The errors (shown as bars) are estimated from the propagation of all errors from counting statistics. 

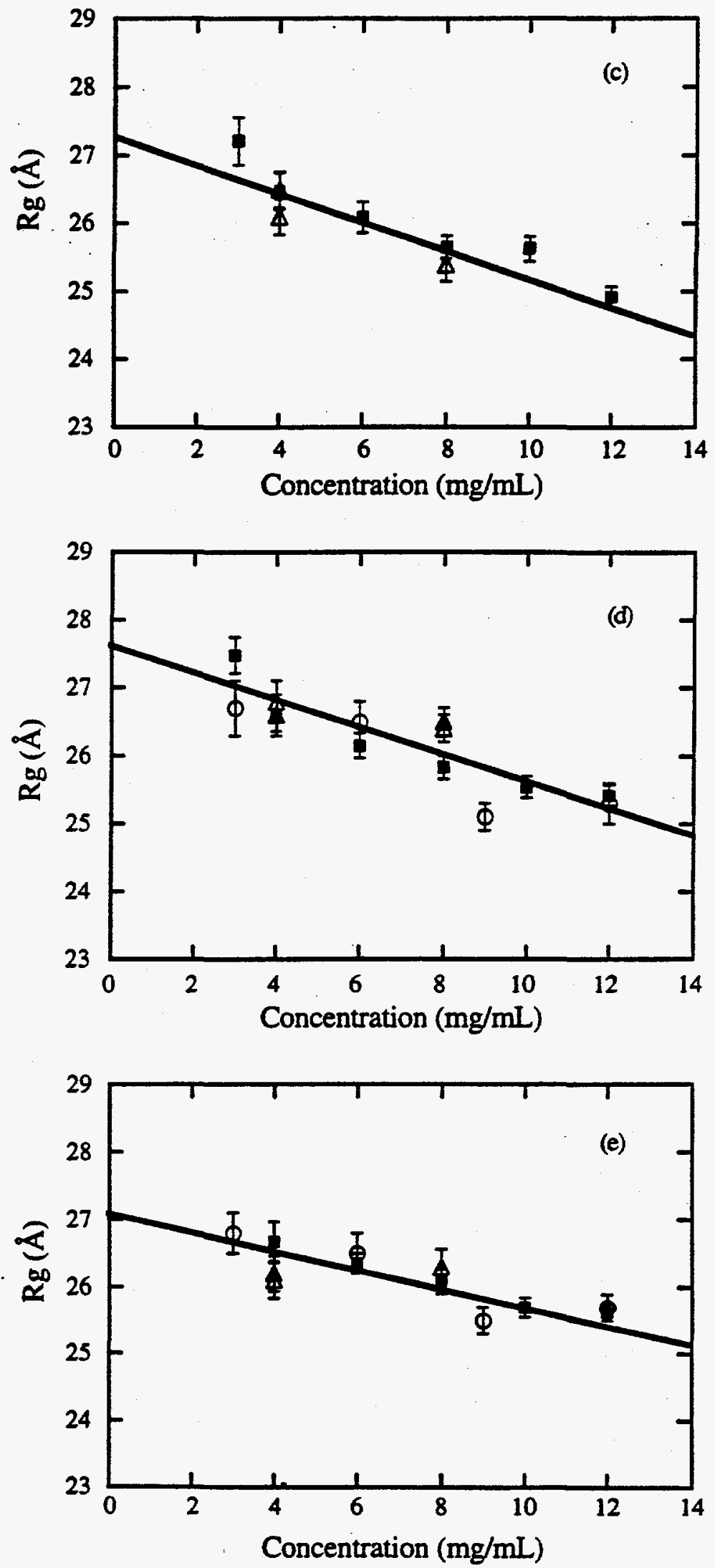
of $\mathrm{Na}_{2} \mathrm{~S}_{2} \mathrm{O}_{4}$ added just before the sample was exposed to $\mathrm{x}$-rays). For example, for the wild-type without nucleotides sample, multiple measurements from two independent runs with flat-window cells gave $\mathrm{R}_{\mathrm{g}}$ of $27.0 \pm 0.3,26.8 \pm 0.3,26.6 \pm 0.3 \AA$ for protein concentration at $4 \mathrm{mg} / \mathrm{ml}$, and $26.1 \pm 0.2,26.1 \pm 0.2,26.2 \pm 0.2 \AA$ for $8 \mathrm{mg} / \mathrm{ml}$. For the wild-type with MgATP sample, $R_{g}$ s were measured to be $25.9 \pm 0.3,25.7 \pm 0.2,25.0 \pm$ $0.3,25.1 \pm 0.2,24.6 \pm 0.3 \AA$ for $4 \mathrm{mg} / \mathrm{ml}$, and $25.9 \pm 0.2,25.4 \pm 0.2,25.2 \pm 0.1,25.0$ $\pm 0.2 \AA$ for $8 \mathrm{mg} / \mathrm{ml}$. Comparable reproducibility was seen for the other three samples. All these data are shown in the Figures 2.4.a-e, from which it is very clear that the observed trends are reproducible and significant.

Changes in $\mathbf{R}_{\mathbf{g}}$ observed upon addition of nucleotides. The final $\mathbf{R}_{\mathbf{g}}$ values obtained from linear least-squares fits of the data points of three runs and subsequent extrapolations of $R_{\mathrm{g}}$ to zero protein concentrations are listed in Table 2.1. There are two major changes for the wild-type Fe protein associated with the binding of MgATP. First, the presence of $\mathrm{MgATP}$ reduces the radius of gyration of wild-type $\mathrm{Fe}$ protein by about 2.0 $\AA$ from $27.3 \pm 0.2 \AA$ to $25.2 \pm 0.2 \AA$. The errors in Table 2.1 represent the statistical errors derived from the linear fit to the data. Although these errors are similar to those reported in similar studies in the literature (see McDonald, et al., 1979;. Pickover, et al., 1979 for examples), our experience leads us to believe that these errors are underestimated due to systematic effects. A reasonable estimate for the maximum error is the biggest error in the individual measurements, in this case $0.5 \AA$ for the $2 \mathrm{mg} / \mathrm{ml}$ samples. The true error probably lies between the values in Table 2.1 and this maximal value. However, even at the worst case of $0.5 \AA$ error, the $2.0 \AA$ difference is still outside the range of experimental error. In addition, a $\sim 1 \AA$ change in $R_{g}$ resulting from protein conformational change is not uncommon in the literature, examples are SAXS studies of the substrate binding of hexokinase and phosphoglycerate kinase (McDonald, et al., 1979; Pickover, et al., 1979). It is therefore concluded that the $2.0 \AA$ decrease in $\mathrm{R}_{\mathrm{g}}$ detected by these measurements is experimentally significant and demonstrates a contraction of the wild-type Fe protein when MgATP is bound.

The second change concerns the dependence of the $\mathbf{R}_{\mathrm{g}}$ on concentration. For four samples, wild-type without nucleotides, wild-type plus MgADP, UW91 without nucleotide and UW91 plus MgATP, there is an apparent decrease in $\mathbf{R}_{\mathbf{g}}$ with increasing protein concentration. As shown in Fig. 2.4.b, the slope of $R_{g}$ versus concentration for the wildtype protein in the presence of MgATP is significantly different from the other four samples. Despite run-to-run systematic errors, the different dependence of $R_{g}$ on 
Table 2.1. Radii of gyration for the wild type and mutant UW91 Fe protein in the absence and presence of nucleotides.

\begin{tabular}{llll}
\hline Fe protein & $\begin{array}{l}R_{g} \text { in the absence of } \\
\text { nucleotides }(\AA)\end{array}$ & $\begin{array}{l}R_{g} \text { in the presence of } \\
\operatorname{MgATP}(\AA)\end{array}$ & $\begin{array}{l}R_{g} \text { in the presence of } \\
\operatorname{MgADP}(\AA)\end{array}$ \\
\hline wild-type Fe protein & $27.3 \pm 0.2$ & $25.2 \pm 0.2$ & $27.2 \pm 0.2$ \\
& & $27.1 \pm 0.2$ & N.A. ${ }^{\mathrm{a}}$ \\
\hline
\end{tabular}

${ }^{a}$ N.A., not applicable.

These data are derived by the Guinier approximation followed by the linear extrapolation of $\mathrm{R}_{\mathrm{g}}$ to zero protein concentration, as shown in Fig. 2.4. (a)-(e). The errors are derived from the least-squares linear extrapolation of $R_{g} v s$. concentration. (No data of mutant UW91 Fe protein in the presence of MgADP were collected because this mutant does not hydrolyze MgATP.) These results are derived from the combined data from all runs. 
concentration for the wild-type Fe protein in the presence of MgATP was observed reproducibly in each independent run, indicating it is not an artifact. This slope will be discussed further later.

Table 2.1 shows that there is no detectable difference in the $\mathrm{R}_{\mathrm{g}}$ observed between the wild-type and the UW91 mutant of Fe protein in the absence of added nucleotides. These data suggest that both proteins are initially in the same conformational state. There is also no detectable difference in the $\mathrm{R}_{\mathrm{g}}$ observed when MgATP is added to the UW91 Fe protein, and when MgADP is added to the wild-type protein. These results confirm the conclusion drawn from chelation experiments that MgATP does not induce a detectable conformational change in the UW91 Fe protein (Gavini \& Burgess, 1992), and that MgADP inhibits the chelation (Walker \& Mortenson, 1973; Walker \& Mortenson, 1974). All these data show that within the sensitivity of the SAXS experiment, the wild-type protein in the absence of nucleotide, the wild-type protein in the presence of MgADP, the UW91 protein in the absence of nucleotide and the UW91 protein in the presence of MgATP adopt a similar conformational state, which is different from that of wild-type protein in the presence of MgATP. More importantly, the lack of conformational change in the Fe protein mutant supports the hypothesis that a proper conformational state needs to be established for electron transfer to occur (Gavini \& Burgess, 1992).

Possible nature of the MgATP-induced conformational change for the wildtype Fe protein. The SAXS measurements described above provide direct evidence that the wild-type Fe protein undergoes an overall contraction in the presence of MgATP. This conformational contraction is likely to be the source of the previously reported changes associated with the environment around [4Fe-4S] cluster following the binding of MgATP. These changes following the MgATP binding have been detected by EPR (Orme-Johnson, et al., 1972; Smith, et al., 1973; Zumft, et al., 1972), electrochemistry studies (Morgan, et al., 1986; Zumft, et al., 1974), chelation studies (Walker \& Mortenson, 1973; Walker \& Mortenson, 1974) as well as oxygen sensitivity experiments.

Considering that the $\mathrm{Fe}$ protein consists of two monomers connected by a [ $4 \mathrm{Fe}-4 \mathrm{~S}$ ] cluster, and that the nucleotide binding sites are at the cleft between the two lobes of the protein molecule (Georgiadis, et al., 1992), the present results could be accounted for by a model involving the hinged movement of the two subdomains of the Fe protein. A hinged motion of two domains of a protein molecule induced by binding substrate has been observed in hexokinase (McDonald, et al., 1979) and phosphoglycerate kinase (Pickover, 
et al., 1979), for example. In the case of hexokinase, the decrease of $\sim 1 \AA$ in $R_{g}$ by SAXS is paralleled by the observations in a crystal structure study of a rotation of $12^{\circ}$ of one lobe relative to the other and a closing of a cleft between two lobes with the movement of polypeptide backbone by $9 \AA$ (McDonald, et al., 1979).

For the Fe protein, the nucleotides are proposed to bind at one subunit with its phosphate groups and with the ribose and adenine extending across the subunit interface to interact with residues of the other subunit (Georgiadis, et al., 1992). The binding of nucleotides might therefore result in narrowing the gap between the subunits. A decrease of $2.0 \AA$ in $\mathbf{R}_{\mathrm{g}}$ may originate from a several $\AA$ reduction in interdomain distance, and probably a rotation between domains. If this explanation is correct, however, the present results show that only MgATP, and not MgADP, can induce the closure of the gap. It may be that the additional contacts formed between protein domains and the $\gamma$-phosphate groups of ATP are required to bring the two subdomains closer to each other. Furthermore, this closure of domains induced by the binding of MgATP has to occur in such a way that the environment around the [4Fe-4S] cluster is altered to a state which is optimally configured to transfer electrons to the MoFe protein. The most straightforward hypothesis would be that the $[4 \mathrm{Fe}-4 \mathrm{~S}$ ] cluster which is more buried in the protein matrix in the absence of MgATP becomes more exposed with the closure of the cleft, making it more accessible. Since it is proposed that the region of the Fe protein which includes the cluster is involved in forming the complex with the MoFe protein for electron transfer (Georgiadis, et al., 1992), it is conceivable that the more exposed [4Fe-4S] cluster facilitates the effective transfer of electrons.

It is shown in Fig. 2.4.b that for the wild-type Fe protein in the presence of MgATP the radii of gyration are nearly independent of concentration. The effect of concentration-dependence of $R_{g} s$ includes both interparticle interference and contamination of trace amounts of higher aggregation states (Hubbard, 1987). The nature of interparticle interference is such that it decreases the scattered intensity in the Guinier region (Glatter, 1982). On the other hand, because the forward intensity is roughly proportional to the square of molecular weight, the presence of higher aggregation states, e.g. dimers, will increase the intensity also in the Guinier region. Thus the lack of $\mathbf{R}_{\mathbf{g}}$ concentrationdependence in the MgATP-bound wild-type protein could result from a cancellation between these two contributions. Since both the interparticle interference and dimerization originate from intermolecular forces, this cancellation may reflect a change in the intermolecular force of the MgATP-bound wild-type protein. The absence of this effect in 
the MgATP-bound mutant UW91 protein further suggests that this change of intermolecular forces in the MgATP-bound wild-type Fe protein might be associated with the conformational change induced by MgATP. It is in any case quite clear that the concentration-dependence of solution aggregation of the Fe protein may be quite different for the MgATP-bound wild type, perhaps reflecting altered surface electrostatic properties.

Finally, we notice that at the highest scattering angle region available for these measurements ( $S=0.025 \AA^{-1}$ ), the scattering intensity from the wild-type $F e$ protein solution in the presence of MgATP is different from those of the other four systems. The higher angle region is sensitive to the internal structure of the protein molecule, e.g. the arrangement of secondary structure (Glatter, 1982). Therefore, it is worthwhile to extend our data collection to higher angle regions. This will enable more reliable use of the indirect transform method to obtain pair distribution $(P(r))$ functions, which have been found to be useful in studying subunit organization (Glatter, 1982). Since the crystal structure of the Fe protein has recently been solved (Georgiadis, et al., 1992), it now becomes feasible to build a molecular model and alter it to investigate the effects of MgATP binding on the SAXS curves and $P(r)$ functions. Such comparisons could provide molecular information on how MgATP triggers the Fe protein conformational change, which in turn is required for electron transfer.

\subsubsection{The MoFe Protein}

As an initial step to investigate the interaction between the MoFe protein and the $\mathrm{Fe}$ protein, scattering of the MoFe protein alone was measured. No sample color change was observed during or after data collection. In the Guinier plot, there is no obvious upswing at the small angle region, indicating that sample was largely aggregation free. Fig. 2.5. shows the concentration dependence of $R_{g}$ for the MoFe protein. An extrapolated value of $39.8( \pm 0.4) \AA$ is obtained, which is in good agreement with $39.2 \AA$ calculated from the atomic coordinates including a $3 \AA$ thick hydration layer, by CRYSOL17. A direct calculation from the coordinates by equation [1], however, gives a much lower value of $31.7 \AA$. 


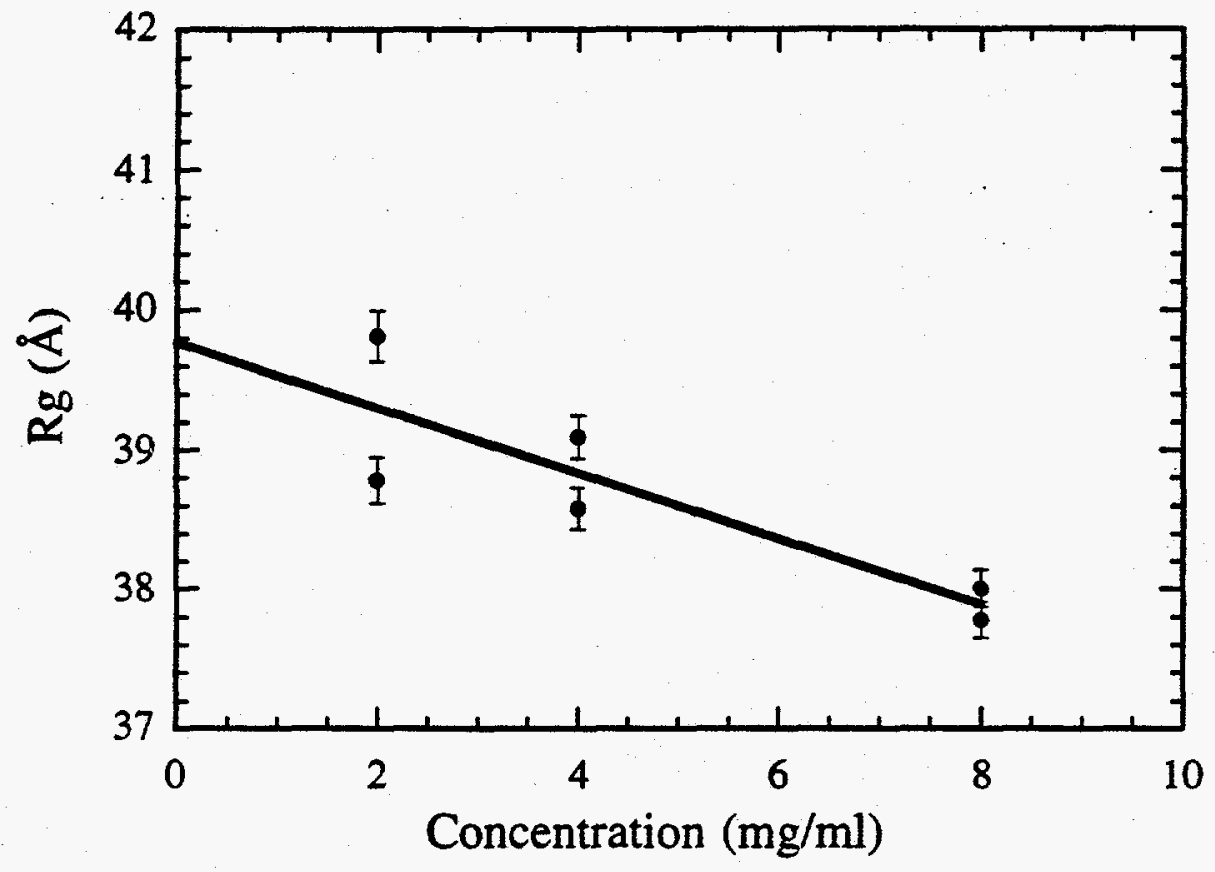

Figure 2.5. $\mathrm{R}_{\mathrm{g}}$ as a function of the $\mathrm{MoFe}$ protein concentration and the extrapolation to zero protein concentration. 


\subsubsection{The FeMo Cluster}

The color of the cluster solution varies from dark brown to light brown depending on the concentration. No sample color change was observed during/after data collection which normally lasted at least 2 hours.

In the first experiment of June 94, two solutions (in MEK and NMF) at a total of six concentrations were tested. For all three concentrations of the MEK solution, a significantly high counting rate was immediately observed when the sample was exposed to $\mathrm{x}$-rays, indicating that the sample formed large aggregates in the solution. Scattering curves resemble those from highly aggregated poly-disperse systems. In Guinier plots, there are no linear regions available. Both the counting rate as well as the scattering curves did not show time dependence over the period of data collection ( $>1$ hour), suggesting that the aggregation was not due to radiation. Taken together, these observations indicate that the cluster form large nonspecific aggregates in MEK solution.

The typical counting rate in the first NMF experiment of June 94 was about 4,000 counts per second, for both cluster-containing samples and for background buffers. Interestingly, after normalizing for incident flux, the scattering curves showed a slight time dependence at the beginning of data collection: Scattering intensity tended to decrease, rather than increase which would be expected for the time- or radiation-dependent aggregation. About 60 minutes later, scattering curves became superimposable. This time dependence trend repeated in all three different concentrations, excluding the possibility of beamline or sample related artifacts. The cluster might still be cold when loaded into the SAXS cell since the sample had been frozen in dry ice. The slightly decrease in scattering intensity may be correlated with the sample warming-up process, which might be accelerated by the exposure to $x$-rays.

Because of the aggregation problem in the MEK solution, only NMF solutions were measured in the run of 1995 . Limited by sample supply, only two concentrations were available. Data of the lower concentration $(463 \mu \mathrm{M})$ appear to be problematic: the time course of scattering decrease was observed again for the first hour; a bubble introduced during loading inside the glove box was seen by $x$-rays during the data collection resulting in a background subtraction problem. Therefore, only data from the concentrated sample (1.44 $\mathrm{mM})$ was analyzed. In this run, the detector was placed 
asymmetrically with respect to the beam allowing data collection over a longer $\mathrm{S}$ range. The use of a longer fitting range for Guinier approximation would minimize the effect of any aggregates in solution on the fitting.

After background subtraction and detector response inhomogeneity correction, for some samples, the scattering intensity around the beamstop was still higher than in the other region. The Guinier plot also showed an incline in the smallest angular range for those samples. Therefore, trace amounts of aggregates may still have been present in the NMF solution. Alternatively, the high residual intensity may result from parasitic scattering. Except for this smallest range, data over a relatively large region was found to be reasonably linear (see Fig. 2.6.). In any case, data at the troublesome region could be excluded in the Guinier analysis, and appeared not to affect analysis results. Fitting results of both runs are summarized in Table 2.2.

The averaged $R_{g}$ from all four measurements is $3.6( \pm 0.4) \AA$, the error being the standard deviation of the four measurements. $\mathrm{R}_{\mathrm{g}}$ calculated from coordinates of the cofactor inside the $\mathrm{MoFe}$ protein is $2.93 \AA$. (The coordinates were taken from Brookhaven Protein Databank, entry PDBIMIN.ENT of the MoFe protein.) $R_{g}$ calculated from coordinates is normally smaller than from the measurement done in solution because of solvation effects. For example, for the Fe protein and the MoFe protein, $R_{g}$ calculated from atomic coordinates by equation [1] is $22.5 \AA$ and $31.7 \AA$, whereas values of 27.2 $( \pm 0.3) \AA$ and $39.8( \pm 0.4) \AA$ respectively are obtained from SAXS. If a hydration layer of $\sim 3 \AA$ thick is considered, better agreements are obtained $(26.5 \AA$ and $39.2 \AA$ for the $\mathrm{Fe}$ protein and the MoFe protein, respectively). A $3.6 \AA \mathrm{R}_{\mathrm{g}}$ in solution is, therefore, rather reasonable for an estimate of $2.93 \AA$ from coordinates without hydration layers, suggesting that the cluster is largely monomeric in NMF. Alternatively, a larger $R_{\mathbf{g}}$ may be correlated with the change in the Mo environment observed by EXAFS study (Ma, et al., 1994). Compared with the FeMo cofactor in NMF solution or in the MoFe protein matrix, the Mo atom in the FeMo cluster has one less $\mathrm{Fe}$ atom and one less N/O atom in the first coordination shell, and does not show a contribution from a long distance of 3 iron atoms in the subcluster $\mathrm{Fe}_{4} \mathrm{~S}_{3}$. Thus, the conformation of the cluster could be different from that of the cofactor. The implication of the loss of an iron atom in the perturbation of the bridge between the two subclusters and in the loss of the long range order, might suggest a partial opening of the structure in the cluster, which would result in a larger $R_{g}$ for the cluster compared with the cofactor. 


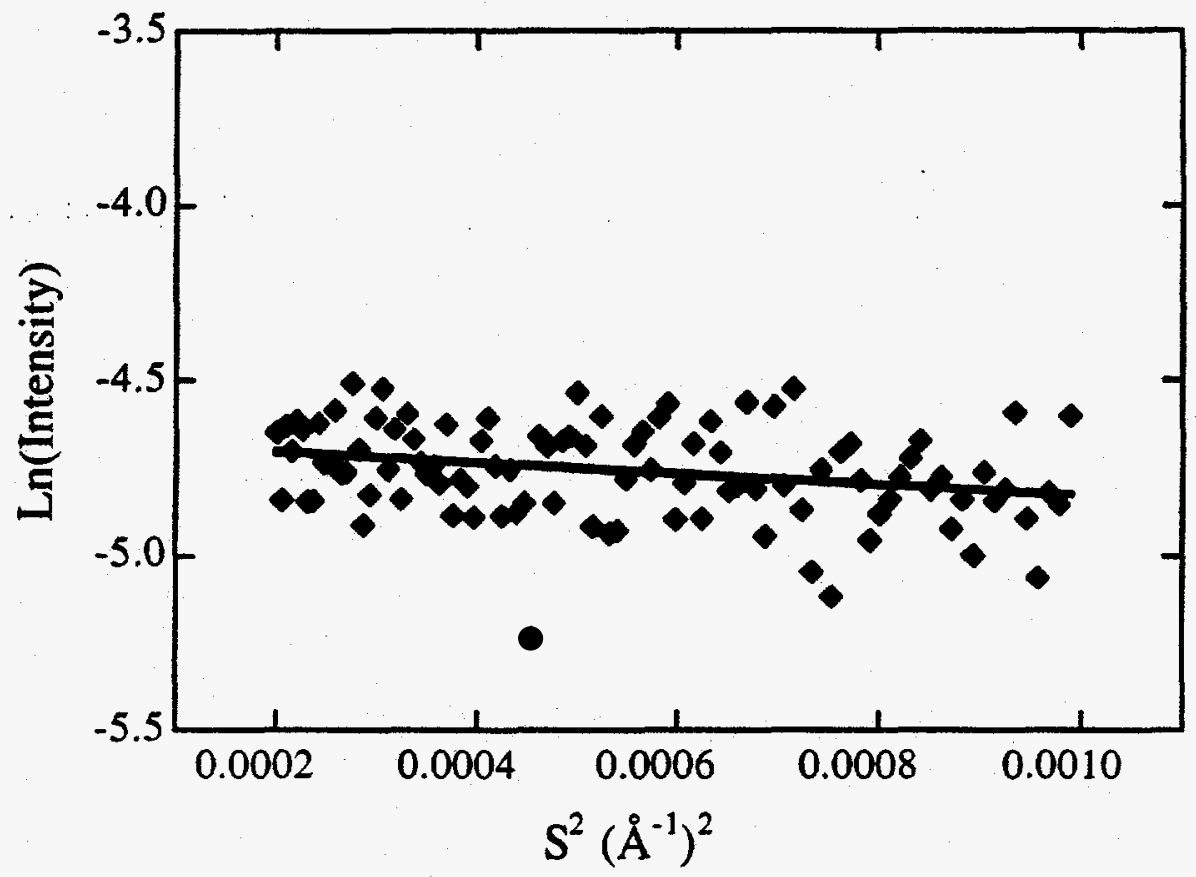

Figure 2.6. A representative Guinier plot of the FeMo cluster in NMF solution and the least-squares fit. The cluster concentration is $464 \mu \mathrm{M}$ (June 94 data). 
Table 2.2. Radii of gyration of the FeMo cluster calculated from Guinier approximation and the fitting range.

\begin{tabular}{llll}
\hline Run & Concentration & $R_{\mathrm{g}}(\AA)$ & $S^{2} \min -S^{2} \max$ \\
\hline June of 94 & $464 \mu \mathrm{M}$ & $3.59 \pm 0.59$ & $0.0002-0.0010$ \\
& $619 \mu \mathrm{M}$ & $4.16 \pm 0.65$ & $0.0002-0.0010$ \\
& $731 \mu \mathrm{M}$ & $3.41 \pm 1.64$ & $0.0004-0.0012$ \\
June of 95 & $1.44 \mathrm{mM}$ & $3.39 \pm 0.22$ & $0.0003-0.0015$ \\
\hline
\end{tabular}


The correlation of the measured $R_{g}$ for the cluster with the estimated $R_{g}$ for the cofactor from the coordinates greatly contrasts with the much higher $R_{g}(6.96 \AA)$ obtained for the cofactor in the same solvent, NMF. A substantially higher intensity at the small angular region was observed in the cofactor samples, leading to a model of coexistence of small oligomers as well as large aggregates: As mentioned in section 2.1.4., compared with the cofactor, the FeMo cluster is obtained differently and exhibits some structural and functional differences. Results from SAXS experiments further demonstrate that the aggregation state in NMF solution also distinguishes the FeMo cluster from the FoMo cofactor. Therefore, it is suggested that some steps in the preparation, perhaps the loss of homocitrate, might be responsible for the change of the aggregation state.

The monodisperse monomeric state of the FeMo cluster also contrasts sharply with the cluster in MEK solution. In the latter, the cluster obviously formed large polydisperse aggregates such that there is no linear region in the Guinier plot. This is not unexpected because it is known that the cluster is more soluble in NMF than in MEK. The cluster can be concentrated up to 2-3 mM in NMF while the maximum concentration in MEK is -1 $\mathrm{mM}$. Considering its small size, a net charge of +2 renders the cluster a highly charged molecule. A much higher dielectric constant, 182.4 at $25^{\circ} \mathrm{C}$ (Riddick \& Bunger, 1970), makes NMF a better solvent for the cluster than MEK, which has a dielectric constant of 18.51 at $20^{\circ} \mathrm{C}$ (Riddick \& Bunger, 1970), which is even lower than $\mathrm{H}_{2} \mathrm{O}, 80.10$ at $20^{\circ} \mathrm{C}$ (Weast, et al., 1988). Obviously, NMF will separate the cluster molecules better, making a monodisperse solution, while the cluster tends to hold together in MEK, leading to polydispersity.

In summary, the FeMo cluster appears to be largely monomeric in NMF with a $\mathrm{R}_{\mathrm{g}}$ of $3.6 \pm 0.4 \AA$. On the contrary, the cluster aggregates significantly in the MEK solution.

\subsection{Acknowledgments}

This work was supported by National Institutes of Health Grants RR-01209 (to K. O. H.) and GM-43144 (to B. K. B.): SSRL operations are funded by the Department of Energy, Office of Basic Energy Sciences. The Biotechnology Program is supported by the National Institutes of Health, Biomedical Research Technology Program, National Center for Research Resources. Additional support is provided by the Department of Energy, 
Office of Health and Environmental Research. I also wish to thank Dr. Narasaiah Gavini for providing the Fe protein and the MoFe protein, and for all the sleepless days and nights on the beamline during those difficult runs, and Dr. Li Ma for the cluster sample preparation. Acknowledgment is extended to Dr. Hirotsugu Tsuruta and Dr. David Eliezer for their support in experimental setup, data acquisition and analysis. I would also like to thank Dr. Patrick Frank for additional sample preparation, and both him and Dr. Britt Hedman for their helpful discussions on the nitrogenase project. Daniel Segel also contributed in the data collection and discussions on the cluster project. Finally, I would like to acknowledge Dr. Michel Koch at EMBL for his advice and discussions during the initial difficult runs, and Dr. Dmitri Sverigun for the program CRYSOL17.

\subsection{References}

Burgess, B. K. (1983). Advances in Nitrogen Fixation Research. The Hague: MartinusNijhoff.

Burgess, B. K. (1990). Chem. Rev., 90, 1377-1406.

Burgess, B. K. (1993). Molybdenum Enzyme, Cofactor and Models. Washington D.C.: American Chemical Society.

Burgess, B. K., Jacobs, D. B., \& Stiefel, E. I. (1980). Biochim. Biophys. Acta., 614, 196-209.

Conradson, S. D., Burgess, B. K., Newton, W. E., Mortenson, L. E., \& Hodgson, K. O. (1987). J. Am. Chem. Soc., 109, 7507-7515.

Eliezer, D., Frank, P., Gillis, N., Newton, W. E., Doniach, S., \& Hodgson, K. O. (1993). J. Biol. Chem., 268(28), 20953-20957.

Gavini, N., \& Burgess, B. K. (1992). J. Biol. Chem., 267, 21179-21186.

Georgiadis, M. M., Komiya, H., Chakrabarti, P., Woo, D., Kornuc, J. J., \& Rees, D. C. (1992). Science, 257, 1653-1659.

Glatter, O. (1982). Small-Angle X-ray Scattering. New York: Academic Press.

Hedman, B., Frank, F., Gheller, S. F., Roe, A. L., Newton, W. E., \& Hodgson, K. O. (1988). J. Am. Chem. Soc., 110, 3798-3805.

Holm, R. H. (1990). Prog. Inorg. Chem., 38, 1.

Hubbard, S. R. (1987) Small-Angle X-ray Scattering Studies of Calcium-Binding Proteins in Solution. Ph.D. Thesis, Stanford University.

Kim, J., \& Rees, D. C. (1992). Science, 257, 1677-1682.

Ljones, T., \& Burris, R. H. (1978). Biochemistry, 17, 1866-1872. 
Ma, L., Gavini, N., Liu, H. I., Hedman, B., Hodgson, K. O., \& Burgess, B. K. (1994). J. Biol. Chem., 269, 18007-18015.

McDonald, R. C., Steitz, T. A., \& Engelman, D. M. (1979). Biochemistry, 18, 338-342.

Morgan, T. V., Prince, R. C., \& Mortenson, L. E. (1986). FEBS Lett., 206, 4-8.

Nielsen, A. (1968). An Investigation of Promoted Iron Catalysts for the Synthesis of Ammonia. Copenhagen: Jul. Giellerup Forlag.

Orme-Johnson, W. H., Hamilton, W. D., Jones, T. L., Tso, M.-Y. W., Burris, R. H., Shah, V. K., \& Brill, W. J. (1972). Proc. Natl. Acad. Sci. USA, 69, 3142-3145.

Pickover, A. C., McKay, D. B., Engelman, D. M., \& Steitz, T. A. (1979). J. Biol. Chem., 254, 11323-11329.

Rawlings, J., Shah, V. K., Chisnell, J. R., Brill, W. J., Zimmerman, R., Munck, E., \& Orme-Johnson, W. H. (1978). J. Biol. Chem., 253, 1001.

Riddick, J. A., \& Bunger, W. B. (1970). Techniques of Chemistry. New York: WileyInterscience.

Robson, R. L. (1984). FEBS Lett., 173, 394-398.

Seefeldt, L. C., Morgan, T. V., Dean, D. R., \& Mortenson, L. E. (1992). J. Biol. Chem., 267, 6680-6688.

Shah, V. K., \& Brill, W. J. (1977). Proc. Natl. Acad. Sci. USA, 74(8), 3249-3253.

Shah, V. K., \& Brill, W. J. (1981). Proc. Natl. Acad. Sci. USA., 78, 3438-3440.

Shah, V. K., Davis, L. C., Gordon, J. K., Orme-Johnson, W. H., \& Brill, W. J. (1973). Biochem. Biophys. Acta, 292, 246-255.

Smith, B. E., Lowe, D. J., \& Bray, R. C. (1973). Biochem. J., 135, 331-341.

Stephens, P. J., McKenna, C. E., McKenna, M., C., Nguyen, H. T., \& Lowe, D. J. (1982). Electron Transport and Oxygen Utilization. New York: Elsevier Biomedical.

Svergun, D. I., Barberato, C., \& Koch, M. H. J. (1995). J. Appl. Crystallogr., submitted for publication.

Thorneley, R. N. F., Ashby, G. A., Fisher, K., \& Lowe, D. J. (1993). Electron-transfer reactions associated with nitrogenase from Klebsiella pneumoninae. In Stiefel, E. I., Coucouvanis, D., \& Newton, W. E. (Eds.), Molybdenum Enzymes, Cofactors and Model Systems (pp. 209-302). Washington, D. C.: American Chemical Society.

Thorneley, R. N. F., \& Lowe, D. J. (1985). Molybdenum Enzymes. New York: Wiley. Tso, M.-Y. W., \& Burris, R. H. (1973). Biochim. Biophys. Acta, 309, 263-270.

Wakatsuki, S., Hodgson, K. O., Eliezer, D., Rice, M., Hubbard, S., Gillis, N., Doniach, S., \& Spann, U. (1992). Rev. Sci. Instrum., 63, 1736-1740. 
Walker, G. A., \& Mortenson, L. E. (1973). Biochem. Biophys. Res. Commun., 53, 904909.

Walker, G. A., \& Mortenson, L. E. (1974). Biochemistry, 13, 2382-2388.

Walker, J. E., Saraste, M., Runswick, M. J., \& Gay, N. J. (1982). EMBO J., 8, 945 951.

Weast, R. C., Astle, M. J., \& Beyer, W. H. (Ed.). (1988). Handbook of Chemistry and Physics (69 ed.). Boca Raton, FL: CRC Press, Inc.

Willing, A., \& Howard, J. B. (1990). J. Biol. Chem., 265, 6596-6599.

Yates, M. G. (1991). Biological Nitrogen Fixation. New York: Chapman and Hall.

Zumft, W. G., Cretney, W. C., Huang, T. C., Mortenson, L. E., \& Palmer, G. (1972). Biochem. Biophys. Res. Commun., 48, 1525-1532.

Zumft, W. G., Mortenson, L. E., \& Palmer, G. (1974). Eur. J. Biochem., 46, 525-535. 
Chapter 3

Solution Small-angle X-ray Scattering Study of the Molecular Chaperone hsc70 and Its Subfragments 


\subsection{Introduction and Background}

In response to unfavorable conditions such as heat shock, living organisms accelerate the rate of expression of some specific genes (heat shock genes); the products of these genes are referred to as heat shock proteins historically or stress proteins. Heat shock response was first observed in 1962 in Drosophila hydei where changes of puffing, or gene activity pattern, were induced by exposing salivary gland polytene chromosomes to evaluated temperature (Ritossa, 1962). However, it was the discovery of heat shock proteins in 1974 (Tissieres, et al., 1974) that stimulated great interest from molecular biologists and launched the study of heat shock response. Since then knowledge in this field has been greatly accumulated and expanded, making the heat shock system one of the most stimulating and prosperous areas in molecular and cellular biology, and more recently in immunology and medicine.

Although heat shock proteins were initially recognized under heat shock condition, it was found later that organisms also enhance synthesis of these proteins in response to other general stress physiology, such as anoxia, chemical poisons and virus infection. It is further demonstrated that most of the heat shock proteins are also present under normal physiological conditions, but with less amount, and that they are crucial for cell viability (Morimoto, et al., 1990). This subset of heat shock proteins, which are constuitively expressed, is referred to as heat shock cognates (hsc) to distinguish them from the stress induced heat shock proteins. The major heat shock proteins are classified into five subfamilies according to the approximate molecular weights (MW): 1) hsp100 with MW larger than $100 \mathrm{kDa}$; 2) hsp90 with MW of $83-90 \mathrm{kDa} ; 3)$ hsp70 with MW of $66-78 \mathrm{kDa}$; 4) hsp60 with $\mathrm{MW}$ of about $60 \mathrm{kDa}$; and 5) small heat shock proteins of 15 $30 \mathrm{kDa}$. Strikingly, heat shock proteins are highly conserved within each class in evolution, for example, hsp 70 family shares about $50 \%$ sequence conservation from $E$. coli to human.

Heat shock proteins were proposed to protect the cell against adverse environmental circumstances, and to facilitate the repair and recovery to normal cellular activities after the stress period (Nover, 1984). More specifically, since most inducers of stress response led to the accumulation of denatured proteins in the cell, heat shock proteins were implicated in functions dealing with this problem by preventing denatured proteins from misfolding or aggregation (Morimoto, et al., 1990). Recent progress in this area reveals a better understanding of the biochemical properties of heat shock proteins 
under both stress and normal conditions. They are involved in regulation of protein folding, protein synthesis, translocation of proteins across membranes, and assembly and disassembly of macromolecular complexes. All of these functions can be ultimately ascribed to the ability of heat shock proteins to recognize and interact with "unstructured" segments present in the other proteins. In particular, because of their roles in protein folding process, heat shock proteins are also called molecular chaperones (Morimoto, et al., 1994).

The $70 \mathrm{kDa}$ heat shock proteins. Members of the $70 \mathrm{kDa}$ heat shock protein family were identified during the first description of heat shock proteins in 1974 (Tissieres, et al., 1974). It was further found that hsp70 are the most abundant heat shock proteins in many organisms with most of them present in unstressed cells. The constutivelyexpressed members, or heat shock cognates, are one of the earliest studied and best characterized heat shock proteins. Bovine heat shock cognate protein hsc 70 was initially isolated and characterized as a protein with an in vitro clathrin uncoating activity (Chappell, et al., 1986; Schlossman, et al., 1984). It has subsequently been implicated in transmembrane targeting of proteins (Chirico, et al., 1988; Deshaies, et al., 1988), and has been found associated with nascent polypeptides during translation (Beckman, et al., 1990; Langer, et al., 1992; Scherer, et al., 1990). In addition, in vitro studies have shown that the $70 \mathrm{kDa}$ heat shock proteins bind both denatured proteins (but not their native counterparts) and some short ( $\geq 7$ residues) synthetic peptides, and that these (poly)peptides are released in response to addition of MgATP (Blond-Elguindi, et al., 1993; Flynn, et al., 1989; Flynn, et al., 1991; Gragerov, et al., 1994).

The $70 \mathrm{kDa}$ heat shock proteins have an ATPase activity, a peptide binding activity, and a coupling mechanism that regulates the peptide binding and release in a nucleotide-dependent manner (Palleros, et al., 1993). For bovine hsc70, the ATPase activity resides in an amino-terminal fragment of about $44 \mathrm{kDa}$ with residues 1 to -386 (Chappell, et al., 1987), and the peptide binding ability lies within an $18 \mathrm{kDa}$ fragment immediately following the ATPase portion (Wang, et al., 1993). A construct consisting of the ATPase fragment and the peptide binding fragment, called the $60 \mathrm{kDa}$ fragment, retains both the ATPase activity and the peptide binding ability (Chappell, et al., 1987). The function of the remainder $\sim 10 \mathrm{kDa}$ with residues 550-650 at the carboxyl terminal is not clear; however, this part of hsc70 has been implicated in interaction with other cellular components. Fig. 3.1 shows the functional arrangements within the primary 


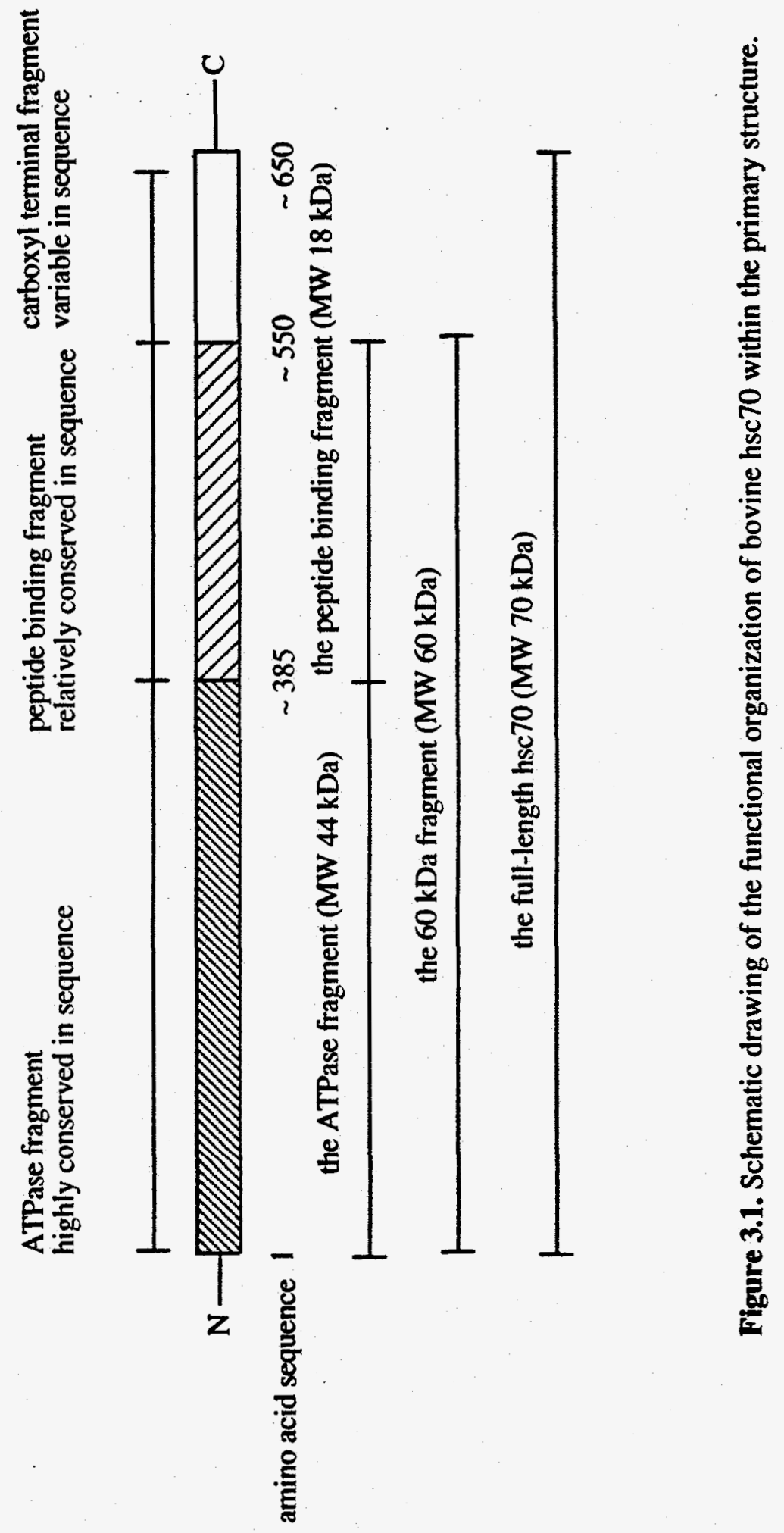


structure of bovine hsc70 (McKay, 1993). Interestingly, although hsp70 is highly conserved in amino acid sequence from bacteria to human, the conservation is not equally distributed over the sequence. As shown in Fig. 3.1, the most highly conserved region is in the ATPase fragment, followed by the less conserved peptide binding fragment. The carboxyl terminal segment is highly variable in sequence.

The turnover rate of ATP by hsp70 is relatively slow: 0.018 (mol ATP $\mathrm{mol}^{-1}$ hsc70 $\mathrm{min}^{-1}$ ) for the bovine hsc70 (Ha \& McKay, 1994), for example. The weak ATPase activity is enhanced severalfolds in the presence of stimulatory peptides. A maximal ATPase rate of $\sim 1.0\left(\mathrm{~mol} \mathrm{ATP} \mathrm{mol}^{-1} \mathrm{hsc} 70 \mathrm{~min}^{-1}\right)$ is reported for the bovine hsc70 in the presence of clathrin light chain peptides (Deluca-Flaherty, et al., 1990). It is interesting to note that this stimulated ATPase activity of the full-length hsc70 is similar to that of the ATPase domain alone, -0.8 ( ${\mathrm{mol} \mathrm{ATP} \mathrm{mol}^{-1} \text { ATPase fragment min }}^{-1}$ ) under identical assay conditions (Deluca-Flaherty, et al., 1990). The C-terminal region including the peptide binding fragment appears to attenuate the ATPase activity of the full-length hsc70; binding of polypeptide substrates somehow restores the activity to a rate characteristic of the ATPase fragment of hsc70 alone. The stimulation of ATPase rate by peptides strongly indicates a close correlation between the ATPase and the peptide binding fragments of hsc 70 .

So far no crystal structures are available for the full-length hsp70 proteins. However, the ATPase domain of bovine hsc 70 has been solved to $2.2 \AA$ resolution, and the structure shows a striking similarity to the tertiary structure of actin (Flaherty, et al., 1990; Flaherty, et al., 1991). Determination of secondary structure of the peptide binding domain of rat hsc70 by NMR has also been reported (Morshauser, et al., 1995).

At least two distinct conformational states have been suggested in hsp70 proteins: one has relatively high affinity for peptides or denatured proteins, while the other has significantly reduced affinity. Proteolysis studies on DnaK, the $E$. coli representative of the hsp70 family, have shown different digestion patterns associated with the presence of MgATP and MgADP (Liberek, et al., 1991). Changes in intrinsic trytophan fluorescence spectra of DnaK bave been observed between MgATP-bound form and MgADP-bound form (Palleros, et al., 1993). The MgADP-bound state of DnaK has a high affinity for denatured protein substrates; the addition of MgATP to the DnaK-MgADP-denatured protein complex leads to a release of the denatured protein substrate and shifts to the MgATP-bound conformation, suggesting a decrease in peptide binding affinity associated 
with the MgATP-bound state (Palleros, et al., 1993). It is further shown in the same study that the conformational transition triggered by MgATP requires the presence of $\mathrm{K}^{+}$.

To develop a low resolution model for hsc70, to determine the magnitude of and the nucleotide requirements for the ATP-induced conformational change, and to attempt to correlate the conformational change with specific steps of the enzymatic ATPase cycle, we have carried out solution small-angle $x$-ray scattering studies of recombinant bovine hsc70 and its subfragments.

\subsection{Experimental Procedures}

\subsubsection{Sample Preparations}

Expression and purification of protein. Full-length recombinant bovine hsc70 (amino acid residues 1-650), which has ATPase and peptide binding activities, and the truncated fragment consisting of residues 1-386 ("ATPase domain") were expressed in $E$. coli as described elsewhere (Wilbanks, et al., 1994). The plasmid for expression of the truncated fragment including residues 1-554 ("60 kDa fragment"), which also has ATPase and peptide binding activities, was subcloned from the pT7-7-derived plasmid used to express hsc70. This was achieved by replacing an EcoRI/Sall fragment including the codons for residues 540 to 650 , termination signals and part of the polylinker with a synthetic oligonucleotide encoding residues 540-554 and a termination codon. Using the same procedure, the truncated fragment encoding residues 388-554 ("peptide binding domain") was subcloned from a pT7-7-derived plasmid that encodes the carboxyl terminal fragment of the protein, residues 388-650. Protein expression was induced in $E$. coli strain BL21(DE3) by addition of isopropylthio- $\beta$-D-galactoside $(0.4 \mathrm{mM}$ final concentration) to log phase cultures.

Purification schemes for hsc70 and the ATPase fragment were modifications of those published previously (O'Brien \& McKay, 1993; Wilbanks, et al., 1994). Cells were lysed by sonication, the lysate was cleared by centrifugation at $-16,000 \mathrm{~g}$ and applied to a DE-52 anion exchange column (Whatman, Clifton, NJ) equilibrated in $25 \mathrm{mM}$ Tris, $25 \mathrm{mM} \mathrm{KCl}, 0.1 \mathrm{mM}$ PMSF, pH 7.0. hsc70 was eluted with $25 \mathrm{mM}$ Tris, $150 \mathrm{mM} \mathrm{KCl}$, $0.1 \mathrm{mM}$ PMSF, pH 7.0; the ATPase fragment was eluted with a gradient of $25-200 \mathrm{mM}$ $\mathrm{KCl}$ in the same buffer. Both proteins were then purified using ATP-agarose (Sigma, St. 
Louis, MO) as published previously, except that hsc70 was applied to ATP-agarose by batch absorption with rocking for 16 hours at $4^{\circ} \mathrm{C}$ rather than by conventional loading of a pre-poured column. Chromatofocusing over a Mono-P column, followed by gelfiltration on a Superdex-75 column, was used as the final step of purification (both columns from Pharmacia, Uppsala, Sweden). Final buffer conditions, established on the gel filtration column, were $10 \mathrm{mM}$ MOPS, $150 \mathrm{mM} \mathrm{KCl}, \mathrm{pH} 7.0$ for the ATPase fragment and $10 \mathrm{mM}$ MOPS, $100 \mathrm{mM} \mathrm{K}(\mathrm{OAc}), \mathrm{pH} 6.5$ for hsc70.

A variation of this protocol was used to purify the $60 \mathrm{kDa}$ fragment. Since it was not retained on the DE-52 column, it was collected in the flowthrough, applied to a hydroxylapatite column (Bio-Rad, Richmond, CA) equilibrated in $20 \mathrm{mM}$ potassium phosphate, $50 \mathrm{mM}$ calcium phosphate, $0.1 \mathrm{mM}$ PMSF, $\mathrm{pH} 7$ and then eluted with a gradient of 20-200 mM potassium phosphate, also with calcium and PMSF at pH 7. Chromatofocusing and gel filtration were carried out as described above; the $60 \mathrm{kDa}$ protein was stored in $10 \mathrm{mM}$ MOPS, $150 \mathrm{mM} \mathrm{KCl}, \mathrm{pH} 7.0$.

Cells expressing the peptide binding domain were harvested and lysed in the same fashion, and the lysate was cleared by centrifugation. The supernatant fraction from that spin was brought to $40 \%$ saturation with $\left(\mathrm{NH}_{4}\right)_{2} \mathrm{SO}_{4}$, the resulting precipitate was removed by centrifugation, the supernatant fraction was brought to $60 \%$ saturation with $\left(\mathrm{NH}_{4}\right)_{2} \mathrm{SO}_{4}$ and the resulting precipitate again removed by centrifugation. The supernatant fraction, containing the majority of the peptide binding domain, was applied to phenyl-sepharose (Sigma, St. Louis, MO) equilibrated in $2 \mathrm{M}\left(\mathrm{NH}_{4}\right)_{2} \mathrm{SO}_{4}, 20 \mathrm{mM}$ bistris propane, $\mathrm{pH} 7$, eluted with $0.6 \mathrm{M}\left(\mathrm{NH}_{4}\right)_{2} \mathrm{SO}_{4}, 20 \mathrm{mM}$ Bis-Tris propane, $\mathrm{pH} 7$, and precipitated by addition of $\left(\mathrm{NH}_{4}\right)_{2} \mathrm{SO}_{4}$ to near saturation. Resuspended protein was filtered and chromatographed by gel-filtration on Superdex-75 in $10 \mathrm{mM}$ MOPS, $100 \mathrm{mM} \mathrm{K}(\mathrm{OAc}), \mathrm{pH} 7$.

Kinetic assays. Kinetic rate constants for ATP hydrolysis, product release, and steadystate ATPase turnover were determined for the $60 \mathrm{kDa}$ fragment at $25^{\circ} \mathrm{C}$, in the presence of $10 \mathrm{mM}$ MOPS, $4.5 \mathrm{mM} \mathrm{Mg}(\mathrm{OAc})_{2}, 150 \mathrm{mM} \mathrm{KCl}, \mathrm{pH} 7$, using methods described previously (Ha \& McKay, 1994).

The rate constant for ATP hydrolysis was determined under single turnover conditions ( 1 or $2 \mu \mathrm{M} 60 \mathrm{kDa}$ fragment, $10 \mathrm{nM} \alpha-[32 \mathrm{P}]$-ATP). Aliquots of the reaction mixture were withdrawn at specific time points, quenched with acid and neutralized, and 
the $\alpha$-32P-labeled adenosine nucleotides were separated by thin layer chromatography. The relative amount of each radioactive nucleotide was determined with a PhosphorImager (Molecular Dynamics, Sunnyvale, CA) system, and the fraction hydrolyzed was computed as the ratio of counts in the ADP spot to the sum of counts in both ADP and ATP spots (after correction for background). Data on the fraction of ATP hydrolyzed versus time were fit with a single exponential, yielding the hydrolysis rate constant.

The rates of release of products were determined using filter binding assays as described previously (Ha \& McKay, 1994). To determine the rate of ADP release, protein was first incubated with stoichiometric amounts of $\alpha-\left\{{ }^{32} \mathrm{P}\right]-\mathrm{ATP}$ for sufficient time to allow complete hydrolysis ( $>6 \times$ hydrolysis half-life). Then, an excess of unlabeled ATP was added, and the amount of label released from the protein was followed as a function of time; concentrations after addition of unlabeled ATP were: 2 $\mathrm{mM}$ protein, $100 \mathrm{mM}$ ATP. Aliquots were withdrawn from the reaction and filtered immediately through BA-85 nitrocellulose membranes (Schleicher \& Schuell, Keene, New Hampshire); filters were then washed briefly with buffer, dried and counted by Cherenkov counting. A rate was inferred from a single exponential fit to the data. To determine the rate of release of $P_{j}$, reactions were initiated by mixing $10 \mathrm{nM}$ of $\gamma-\left[{ }^{32} \mathrm{P}\right]-$ ATP with $10 \mathrm{mM}$ of protein, and the release of ${ }^{32} \mathrm{P}_{\mathrm{j}}$ was monitored by filter binding. The rate constant for $P_{i}$ release was extracted from the data using methods described previously which take into account the effect of ATP hydrolysis on the apparent release rate (Ha \& McKay, 1994).

Steady state hydrolysis rates were determined in reactions containing 40 or $400 \mathrm{nM}$ enzyme, $4 \mu \mathrm{M}{ }^{32} \mathrm{P}-\alpha$-ATP in the same buffer as for single turnover reactions. Separation and measurement of ATP and ADP were by TLC and PhosphorImager as described above. The hydrolysis rate was computed as the slope of a linear fit to data representing the fraction of ATP hydrolyzed versus time.

Preparation of protein samples for solution scattering. hsc70 and the peptide binding domain aggregate significantly when left in solution at high $(-10 \mathrm{mg} / \mathrm{ml})$ concentrations for an extended period of time (-days). To circumvent this problem, samples of these proteins used for scattering measurements were rendered aggregate-free by gel filtration within 24 hours of their use, and measurements were made at concentrations less than 4 $\mathrm{mg} / \mathrm{ml}$ (for hsc70) or $8 \mathrm{mg} / \mathrm{ml}$ (peptide binding fragment). This precaution was not 
necessary for the ATPase and $60 \mathrm{kDa}$ fragments, since they had less propensity to aggregate. Protein concentrations were determined spectrophotometrically, using calculated molar extinction coefficients at $\lambda=280 \mathrm{~nm}$ of 30,$800 ; 23,700 ; 18,600$ and 5,100 $\left(M^{-1} \mathrm{~cm}^{-1}\right)$ for hsc70, the $60 \mathrm{kDa}$ fragment, ATPase fragment, and peptide binding domain, respectively. The molecular weighs of these species were calculated to be 71.2 ; $61.0 ; 42.5$ and $18.4 \mathrm{kDa}$, respectively. Samples were judged to be nucleotide-free based on an $A_{280} / A_{260}$ ratio of $>1.4$. Where necessary, nucleotide was removed from protein by charcoal extraction as described previously (Ha \& McKay, 1994).

Non-denaturing polyacrylamide gels were $7 \%$, with $4 \%$ glycerol present (Kim, et al., 1992). Dynamic light scattering was measured on a Biotage dp801 Molecular Size Detector using samples filtered through $0.02 \mu \mathrm{m}$ filters.

The above sample preparations and kinetic assays were performed by Dr. Sigurd Wilbanks at Prof. David McKay's laboratory at the Department of Structural Biology, Stanford Medical School.

Standard protein samples used in this study. Bovine serum albumin (from Sigma, St. Louis, MO), was dissolved in $50 \mathrm{mM}$ Na-phosphate, $150 \mathrm{mM} \mathrm{NaCl}, \mathrm{pH} 8.0$ and further purified by gel filtration using Superose 6 (Pharmacia, Uppsala, Sweden). The solution was concentrated with Diaflo ultrafilter PM30 (Amicon, Danvers, MA) at 40 psi. The final protein concentration was determined to be $18.8 \mathrm{mg} / \mathrm{ml}$ using $\varepsilon_{280}=3.96 \times 104 \mathrm{M}^{-1}$ $\mathrm{cm}^{-1}$.

Chicken egg-white lysozyme (Sigma, St. Louis, MO) was dialyzed against $40 \mathrm{mM} \mathrm{NaOAc}, 150 \mathrm{mM} \mathrm{NaCl}, \mathrm{pH} 3.8$, and the protein concentration was determined spectrophotometrically using $\varepsilon_{280}=2.64 \mathrm{mg}^{-1} \mathrm{~cm}^{-1} \mathrm{ml}$.

\subsubsection{X-ray Scattering Data Collection}

X-ray solution scattering experiments were performed on beamline 4-2 of the Stanford Synchrotron Radiation Laboratory in March 1994, June 1994, and March 1995 using the resident SAXS camera (Wakatsuki, et al., 1992). The x-ray source (SPEAR) was operated at an energy of $3.0 \mathrm{GeV}$ and a current of $60-100 \mathrm{~mA}$. The synchrotron beam was monochromatized (to an energy of $8980 \mathrm{eV}$ ) by a double crystal Si [111] monochromator. The beamsize at the sample position was $1.0 \mathrm{~mm}(\mathrm{v}) \times 2.1 \mathrm{~mm}(\mathrm{~h})$. A 
Pt-coated mirror was used to reject higher order harmonics in the March 1994 and June 1994 runs, and additionally as a focusing mirror in the March 1995 run. Incident beam intensity was monitored using an ionization chamber positioned immediately before the sample chamber. Both a linear detector (a one-dimensional position-sensitive proportional counter; manufactured by Bio-Logic, Grenoble, France) and a quadrant detector (developed at the EMBL, Grenoble) were used in separate experiments. The linear detector was filled with $80 \%$ xenon $20 \%$ carbon dioxide and was operated at 1.8 1.9 kilovolts during the experiments; the guadrant detector was filled with $70 \%$ argon $30 \%$ carbon dioxide and was operated at 3.4 kilovolts. Sample-to-detector distance was determined from the diffraction pattern of a cholesterol myristate powder sample, and was found to be $234 \pm 1 \mathrm{~cm}$ for the linear detector and $238 \pm 1$ for the quadrant detector. The linear detector covered the scattering range $0.0002<S<0.003 \AA^{-1}$ (S = $2 \sin \theta / \lambda$, where $2 \theta=$ scattering angle and $\lambda=x$-ray wavelength) and the quadrant detector covered $0.0005<\mathrm{S}<0.004 \AA^{-1}$. Detector response was calibrated every six to twelve hours using a homogenous field of radiation, either from copper fluorescence (for the linear detector) or an ${ }^{55} \mathrm{Fe}$ source (for the quadrant detector).

Sample cells were Kel-F or Macor (Corning, Corning, NY) blocks with a 25-30 $\mu$ l sample chamber, $1.2 \mathrm{~mm}$ path length and $10 \mu \mathrm{m}$ thick mica windows. The temperature of the cell was controlled by circulating ethylene glycol from a temperature-controlled bath through the jacket of the cell holder.

During the March 1994 and June 1994 runs, data collection from each sample was limited to 10 minutes, divided into either 5 cycles of 2 minutes. or 10 cycles of 1 minute, since cumulative exposure times significantly greater than 10 minutes resulted in an upswing of the scattering curve at low angle, indicative of radiation-induced sample deterioration. During the March 1995 run, it was found that deterioration could be retarded by addition of $20 \mathrm{mM} \beta$-mercaptoethanol to the protein samples, allowing data collection time to be extended to 20 minutes. Background scattering was recorded either immediately before or immediately after each protein sample using the same sample cell filled with the corresponding buffer and nucleotide solution. Multiple samples were used under each set of conditions, and their results were averaged, in order to improve statistical precision in the final results. Representative samples that were recovered and assayed for ATPase activity after data collection were found to be fully enzymatically active. 


\subsection{Data Analysis}

\subsubsection{Data Reduction and Analysis}

The SSRL software ANOM was used for data reduction (Rice \& Wakatsuki, 1991). Each data cycle was normalized to the incident beam intensity and examined for obvious anomaly, such as failure of the system to record the data, or radiation-induced aggregation; anomalous sets of data amounted to fewer than $0.5 \%$ of all data sets and were discarded. Repetitive cycles of data collected on each sample were averaged and the measured background scattering profile was subtracted. A detector response correction was applied as a channel-by-channel division of the data by the detector response (integrated intensity for each channel) to a uniform field of radiation. For data collected with the linear detector, the two scattering curves from opposite sides of the direct beam were averaged.

Radii of gyration $\left(\mathrm{R}_{\mathrm{g}}\right)$ were extracted from linear detector data with two different methods. First, a value of $\mathrm{R}_{\mathrm{g}}$ was computed using the Guinier approximation (Glatter \& Kratky, 1982),

$$
\operatorname{LnI}(S)=\operatorname{LnI}(0)-\frac{4 \pi^{2} R_{g}^{2}}{3} S^{2}
$$

where $I(S)$ is the scattered intensity at $S$, and $I(0)$ is the intensity at zero angle. This equation was fit to data over a range of $0.0032<S<0.0065 \AA^{-1}$ for samples of hsc 70 or $60 \mathrm{kDa}$ fragment, and over $0.0032<\mathrm{S}<0.0095 \AA^{-1}$ for samples of ATPase fragment or peptide binding domain.

Second, the pair distribution function

$$
P(r)=8 \pi r \int_{0}^{\infty} S I(S) \sin (2 \pi S r) d S
$$

was calculated using the indirect transformation method of Svergun, as implemented in the GNOM program package (Semenyuk \& Svergun, 1991), with data over the angular range $0.0028<S<0.0280 \AA^{-1}$. $R_{g} s$ were also evaluated from the $P(r)$ functions: 


$$
R_{g}^{2}=\frac{\int_{0}^{\infty} P(r) r^{2} d r}{\int_{0}^{\infty} P(r) d r}
$$

Data collected to higher angle on the quadrant detector were combined with data from equivalent samples collected on the linear detector by linearly scaling the two separate data sets to achieve the best fit in the range $0.008<\mathrm{S}<0.012 \AA^{-1}$. The $\mathrm{P}(\mathrm{r})$ function was then computed from the combined scattering curve with the GNOM program, using data over the angular range $0.0026<\mathrm{S}<0.0280 \AA^{-1}$. This function was used in the modeling studies.

In a monodisperse protein solution with a constant electron density contrast, the forward scattering intensity, $I(0)$, is proportional to the square of the molecular weight $(M W)$ of the protein molecule, $I(0) \propto C(M W)^{2}$, where $C$ is protein concentration in molar concentration [M] (Glatter \& Kratky, 1982). In a solution where a fraction $(\alpha)$ of protein molecules dimerize, the concentrations of the protein molecule in monomer (MW) and dimer $(2 \mathrm{MW})$ forms are $(1-\alpha) \mathrm{C}$ and $(\alpha / 2) \mathrm{C}$ respectively. Hence $\mathrm{I}(0) \propto\left\{(1-\alpha) \mathrm{C}(\mathrm{MW})^{2}+\right.$ $\left.(\alpha / 2) \mathrm{C}(2 \mathrm{MW})^{2}\right\}$ or $\mathrm{I}(0) \propto(1+\alpha) \mathrm{MW}^{2}$. For example, if $30 \%$ of protein monomers form dimers, a $30 \%$ increase in $\mathrm{I}(0)$ will be observed.

\subsubsection{Computing Scattering Curves from Protein Coordinates}

A scattering curve is computed from the atomic coordinates for the ATPase domain with the program CRYSOL (Svergun, et al., 1995) since the structure of this domain has been solved. A $3 \AA$ thick hydration layer, with a value of $0.03 \mathrm{e}^{-1} / \AA^{3}$ as the electron density contrast between this hydration layer and the bulk buffer, is included in the computing.

\subsubsection{Computing Scattering Curves from Some Simple Triaxial Bodies}

An initial and rudimentary approach to the shape analysis of the scatterer (protein molecule) is to model the molecule as some simple triaxial bodies. When the protein molecule consists of several domains or subunits, each domain can be modeled 
individually, and the whole molecule is thus composed of several triaxial bodies in certain orientations. Since solution scattering is a low resolution technique, these rather crude models appear quite satisfactory to approximate the shapes of some protein molecules.

The scattering amplitude of some simple triaxial bodies, such as sphere, ellipsoid, cylinder and prism, can be computed analytically for any orientation (Glatter \& Kratky, 1982). However, the spatial averaging, i.e., the integration, has to be performed numerically. In the case of multibody modeling, the scattering amplitudes of individual domain are calculated analytically and added according to the particular orientation in the model. The scattering intensity is obtained by squaring the total amplitude and averaging over all orientations.

The following analysis is based on the model of prisms since rectangular prisms were used as rudimentary models for hsc70 and its subfragments in this study. For other triaxial bodies, the analysis is similar: only the scattering amplitude, F, needs to be replaced accordingly.

The scattering amplitude of a prism with edge lengths A, B, C is (Hiragi \& Ihara, 1981):

$$
\mathrm{F}(\mathrm{A}, \mathrm{B}, \mathrm{C}, \mathrm{S}, \theta, \varphi)=8 \mathrm{ABC}\left[\frac{\sin \left(\mathrm{As}_{1} \cos \varphi\right)}{\mathrm{As} s_{1} \cos \varphi}\right]\left[\frac{\sin \left(\mathrm{Bs}_{1} \sin \varphi\right)}{\mathrm{Bs}_{1} \sin \varphi}\right] \Psi_{\mathrm{c}}
$$

where:

$$
\begin{aligned}
& \mathrm{s}_{1}=\mathrm{S} \sin \theta ; \\
& \mathrm{s}_{2}=\mathrm{S} \cos \theta ; \\
& \Psi_{\mathrm{c}}=\sin \left(\mathrm{Cs}_{2}\right) /\left(\mathrm{Cs}_{2}\right) ;
\end{aligned}
$$

$\theta$ and $\varphi$ are the zenith and azimuth angles.

The scattering intensity of a prism is thus as follows: 


$$
\begin{aligned}
I(S) & =\left(V^{2} \bar{\rho}^{2}\right) \frac{\int_{0}^{2 \pi} \int_{0}^{\pi} F(A, B, C, S, \theta, \varphi) F^{*}(A, B, C, S, \theta, \varphi) \sin \theta d \theta d \varphi}{\int_{0}^{2 \pi} \int_{0}^{\pi} \sin \theta d \theta d \varphi} \\
& =\left(V^{2} \bar{\rho}^{2}\right) \int_{0}^{2 \pi} \int_{0}^{\pi} F^{2}(A, B, C, S, \theta, \varphi)(\sin \theta / 4 \pi) d \theta d \varphi \\
& =V^{2} \int_{0}^{2 \pi} \int_{0}^{\pi} F^{2}(A, B, C, S, \theta, \varphi)(\sin \theta / 4 \pi) d \theta d \varphi
\end{aligned}
$$

where $\mathrm{V}$ is the volume of the prism, $\mathrm{V}=\mathrm{ABC}$, and $\bar{\rho}$ is the average excess scattering density. For simplicity, $\bar{p}$ is taken as 1 .

For the complex model of $\mathrm{i}$ prisms with scattering amplitude $\mathrm{F}_{\mathrm{i}}$ 's, the scattering intensity can be decomposed into two terms (Fujisawa, et al., 1990). In the first term, the calculation of the scattering intensity from each prism is performed independently in its local axes by equation [5], and the contribution from individual prism weighted by the excess scattering mass is added together. The second term concerns the interference between different prisms. The orientation of each prism can be viewed as a rotation of the local axes $\left(\theta_{\mathrm{i}}, \varphi_{\mathrm{j}}\right)$ with respect to the global axes $(\Theta, \Phi)$ via three Euler angles $\left(\alpha_{\mathrm{j}}, \beta_{\mathrm{j}}\right.$, $\gamma_{i}$ ). Local axes are related to the global axes by:

$$
\left(\begin{array}{l}
\sin \theta \cos \varphi \\
\sin \theta \sin \varphi \\
\cos \theta
\end{array}\right)=\mathrm{T}\left(\alpha_{\mathrm{i}}, \beta_{\mathrm{i}}, \gamma_{\mathrm{i}}\right)\left(\begin{array}{l}
\sin \Theta \cos \Phi \\
\sin \Theta \sin \Phi \\
\cos \Theta
\end{array}\right)
$$

where $T\left(\alpha_{i}, \beta_{i}, \gamma_{i}\right)$ is the orthogonal transformation matrix:

$\mathrm{T}\left(\alpha_{\mathrm{i}}, \beta_{\mathrm{i}}, \gamma_{\mathrm{i}}\right)=\left(\begin{array}{ccc}\cos \alpha_{\mathrm{i}} \cos \beta_{\mathrm{i}} \cos \gamma_{\mathrm{i}} & \sin \alpha_{\mathrm{i}} \cos \beta_{\mathrm{i}} \cos \gamma_{\mathrm{i}}+\cos \alpha_{\mathrm{i}} \sin \gamma_{\mathrm{i}} & -\sin \beta_{\mathrm{i}}+\cos \gamma_{\mathrm{i}} \\ -\cos \alpha_{\mathrm{i}} \cos \beta_{\mathrm{i}} \sin \gamma_{\mathrm{i}} & -\sin \alpha_{\mathrm{i}} \cos \beta_{\mathrm{i}} \sin \gamma_{\mathrm{i}}+\cos \alpha_{\mathrm{i}} \cos \gamma_{\mathrm{i}} & \sin \beta_{\mathrm{i}} \sin \gamma_{\mathrm{i}} \\ \cos \alpha_{\mathrm{i}} \sin \beta_{\mathrm{i}} & \sin \alpha_{\mathrm{i}} \sin \beta_{\mathrm{i}} & \cos \beta_{\mathrm{i}}\end{array}\right)$

The total scattering intensity from a complex model of $i$ prisms can be written as: 


$$
\begin{aligned}
I(S)= & \sum_{j}\left(V_{i}^{2} \bar{\rho}_{i}^{2}\right) \int_{0}^{2 \pi} \int_{0}^{\pi} F_{i}^{2}(A, B, C, \theta, \varphi)(\sin \theta / 4 \pi) d \theta d \varphi \\
& +2 \sum_{i} \sum_{j \neq i}\left(V_{i} \bar{\rho}_{i}\right)\left(V_{j} \bar{\rho}_{j}\right) \int_{0}^{2 \pi} \int_{0}^{\pi} F_{i}(A, B, C, \theta, \varphi) F_{j}(A, B, C, \theta, \varphi) \\
& \times \cos \left(2 \pi S r_{i j} \sin \Theta \cos \Phi\right)(\sin \Theta / 4 \pi) d \Theta d \Phi
\end{aligned}
$$

where $r_{i j}$ is the distance between prism $i$ and $j$. Again, $\bar{\rho}_{i}, \bar{\rho}_{j}$ can be taken as 1 for simplicity.

In practice, the center of one of the prisms is taken as the origin of the global axes, and its local axes are lined up with the global axes.

The pair distribution $(P(r))$ function can be calculated analytically only for a few simple triaxial bodies (Glatter \& Kratky, 1982). In this study, the $P(r)$ functions were obtained from the computed scattering curves, either from equation [5] for the single body model or from equation [8] for the multi-body model, with indirect transformation method implemented in the GNOM program (Semenyuk \& Svergun, 1991).

Radius of gyration can be derived from the computed scattering curves by the Guinier approximation, equation [1], or from the $P(r)$ function, equation [3]. A direct calculation for a single body prism with edge lengths $A, B, C$ is:

$$
R_{g}^{2}=\frac{A^{2}+B^{2}+C^{2}}{12}
$$

$\mathrm{R}_{\mathrm{g}}$ for the multi-body model is derived from parallel axis theorem:

$$
R_{g, \text { total }}^{2}=f_{1} R_{g 1}^{2}+f_{2} R_{g 2}^{2}+f_{1} f_{2} d^{2}
$$

where $f_{1}, f_{2}$ are the mass fractions and $R_{g_{1}}, R_{g_{2}}$ are the radii of gyration of prism 1 and prism 2, $d$ is the distance between their centers of mass. Results from these three calculations should agree well. 


\subsection{Results}

\subsubsection{The Static Measurements}

\subsubsection{A Standard Globular Protein - BSA}

Bovine serum albumin (BSA) is a frequently used globular protein with molecular weight (MW) of $66 \mathrm{kDa}$. BSA was chosen as a model protein initially for the purpose of testing the applicability of the quadrant detector in solution scattering, particularly the feasibility of merging data sets from the linear detector and the quadrant detector for computing the $\mathrm{P}(\mathrm{r})$ function. Because of the similarity in molecular weight, BSA is also a good reference in the size and shape analysis of the $60 \mathrm{kDa}$ fragment and hsc 70 proteins.

The scattering curves were recorded using both the linear and quadrant detectors. $\mathrm{R}_{\mathrm{g}}$ sere calculated using the Guinier approximation, equation [1] applied to both linear and quadrant detector data. Fig. 3.2 shows the concentration dependence of $R_{g}$. The extrapolated value for $R_{g}$ with the linear detector is $29.7( \pm 0.2) \AA$, which is in good agreement with $29.8 \AA$ from literature (Anderegg, et al., 1955).

The quadrant detector is known for improving the signal-to-noise ratio at high angular range which is necessary for computing the $P(r)$ function. To test the reliability of the quadrant detector for solution scattering, as compared with the traditionally used linear detector, scattering data from BSA were also collected using a quadrant detector. Both the $\mathrm{R}_{\mathrm{g}}$ at the individual protein concentration and the extrapolated value $(30.0 \pm 0.2$ $\AA$ ) using the quadrant detector are comparable to those using the linear detector (Fig. 3.2.a, b). Furthermore, the scattering curves with the quadrant detector superimpose satisfactorily with those using the linear detector (Fig. 3.3). Improved statistics with the quadrant detector is apparent from the figure in the wide angle region. However, in practice, the alignment of the quadrant detector to the direct beam is not trivial, and any slight imprecision in alignment may lead to distortions in the scattering profile predominantly occurring in the small angle region. Thus, by employing two detectors, it is reasonable to construct a complete model scattering curve where the low angle portion comes from data with the linear detector, and the wide angle part is taken from data using the quadrant detector. This combined scattering curve is then used to compute the $P(r)$ function, equation [2]. 

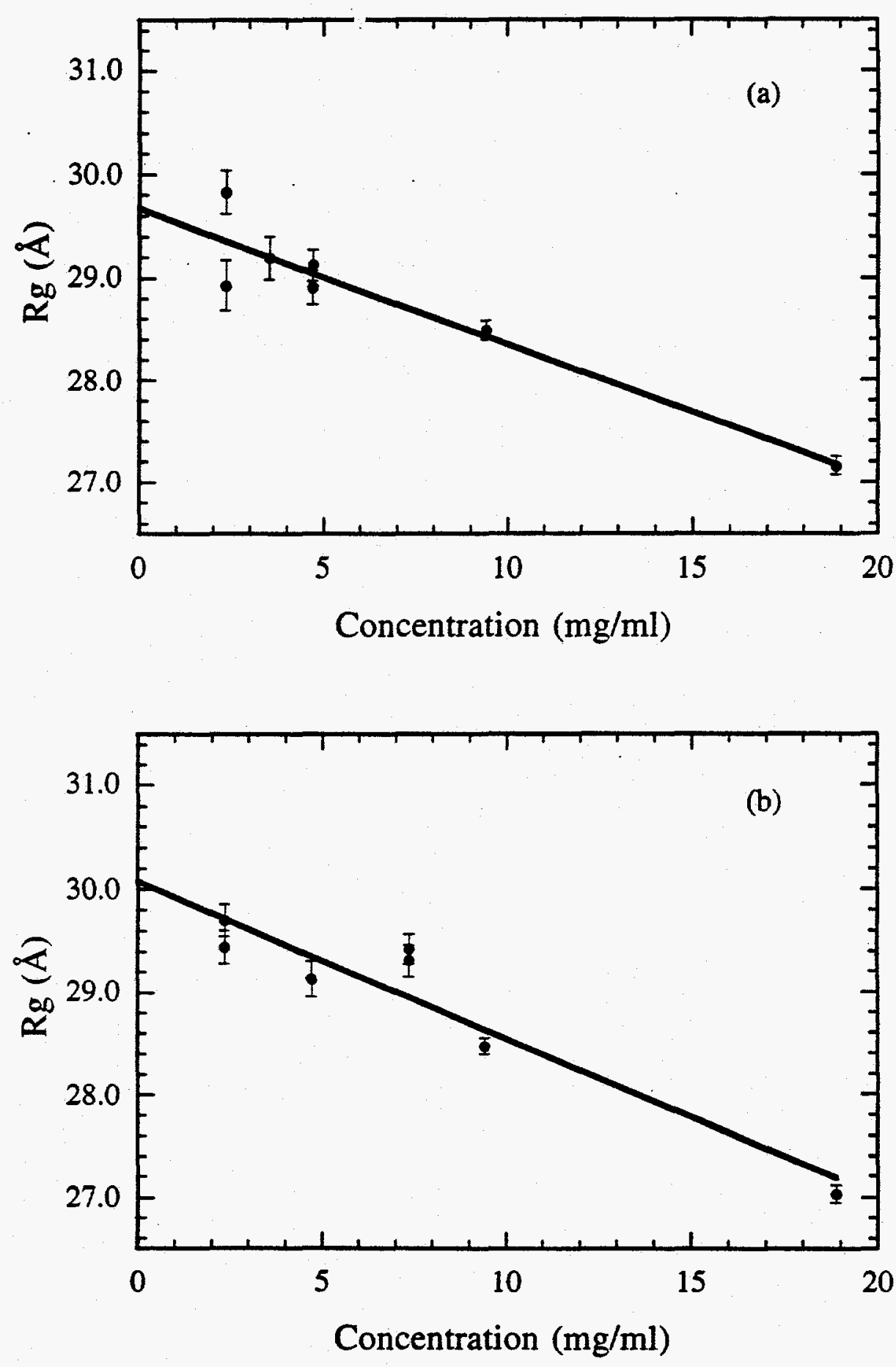

Figure 3.2. Concentration dependence of $R_{g}$ and the extrapolation of $R_{g}$ to zero protein concentration for BSA. a) with the linear detector; $b$ ) with the quadrant detector. 


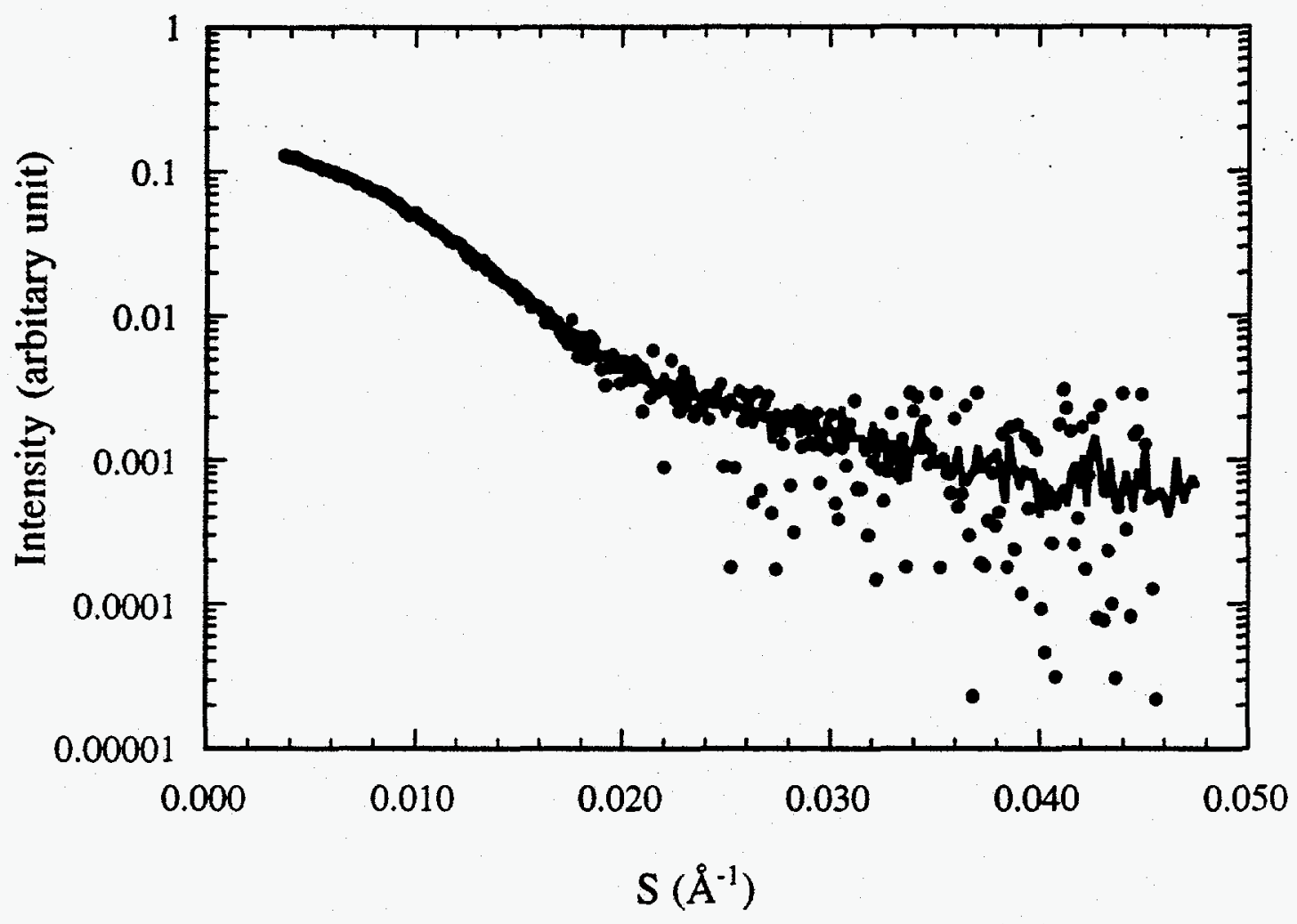

Figure 3.3. Representative scattering curves of BSA recorded with the linear detector $(-)$ and the quadrant detector ( - ). The scattering curves are scaled to achieve the best fit in the range of $0.008<\mathrm{S}<0.012 \AA^{-1}$. The concentration for both measurements was 4.7 $\mathrm{mg} / \mathrm{ml}$. 
A model scattering curve is produced by combining the lir ₹ar detector data of 4.7 $\mathrm{mg} / \mathrm{ml}$ sample with the quadrant detector data of $18.9 \mathrm{mg} / \mathrm{ml}$ sample. The merging range was around $0.017 \AA^{-1}$ in $\mathrm{S}$, beyond which the interparticle interference in the high concentration $(18.9 \mathrm{mg} / \mathrm{ml})$ sample was negligible, and could be neglected. The integrated scattering profile is shown in Fig. 3.4.a. Fig. 3.4.b shows the $P(r)$ function calculated with the GNOM program (Semenyuk \& Svergun, 1991) using this combined data set. $R_{g}$ derived from the $P(r)$ function [3] is $29.6( \pm 0.1) \AA$, in excellent agreement with the extrapolated $R_{g}(29.7 \pm 0.2 \AA)$ from the Guinier approximation. The maximum length of the BSA molecule, $D_{\max }$, is $95( \pm 5) \AA$, and the most populated distance, measured by the peak position, is $38 \AA$. The shape of the $P(r)$ function is typical for a globular shape molecule.

\subsubsection{The Guinier Analysis on hsc70 and Its Subfragements}

Sample integrity. Fig. 3.5 shows the typical small angle $x$-ray scattering curves of the $60 \mathrm{kDa}$ fragment. No significant nonspecific aggregation was observed for all the samples tested, as indicated by a lack of an upswing in the small angle region in the Guinier plot. The forwarded scattering intensity $\mathrm{I}(0)$, which is proportional to the protein molecular weight, was obtained using the Guinier approximation, equation [1]. None of the samples (hsc70, the $60 \mathrm{kDa}$ fragment, the ATPase domain, the C-terminal domain, under various conditions tested) show significant concentration dependence of $I(0)$, suggesting that protein molecules maintained the same aggregation state throughout the concentration range tested. The normalized $\mathrm{I}(0)$ values, $\mathrm{I}(0) / \mathrm{C}$, for the $60 \mathrm{kDa}$ fragment are plotted in Fig. 3.6.

To evaluate the molecular weights of the samples, scattering data were also collected on lysozyme solutions during the measurements. Assuming the same partial specific volume for the hsc70 samples and lysozyme, molecular weights were estimated by comparing the ratios of $\mathrm{I}(0) / \mathrm{C}$ of the hsc70 samples and that of lysozyme. The results are in reasonable agreement with calculations from amino acid residues, suggesting that the protein molecules were monomeric in solution. For example, MWs obtained from SAXS are $\sim 72 \mathrm{kDa}, \sim 54 \mathrm{kDa}$ and $\sim 40 \mathrm{kDa}$ for hsc70, the $60 \mathrm{kDa}$ fragment and the ATPase domain, while calculations from amino acid residues give $71.2 \mathrm{kDa}, 61.0 \mathrm{kDa}$ and $42.5 \mathrm{kDa}$ respectively. Altogether, samples in our scattering studies were largely aggregation free, and in monomeric form. 

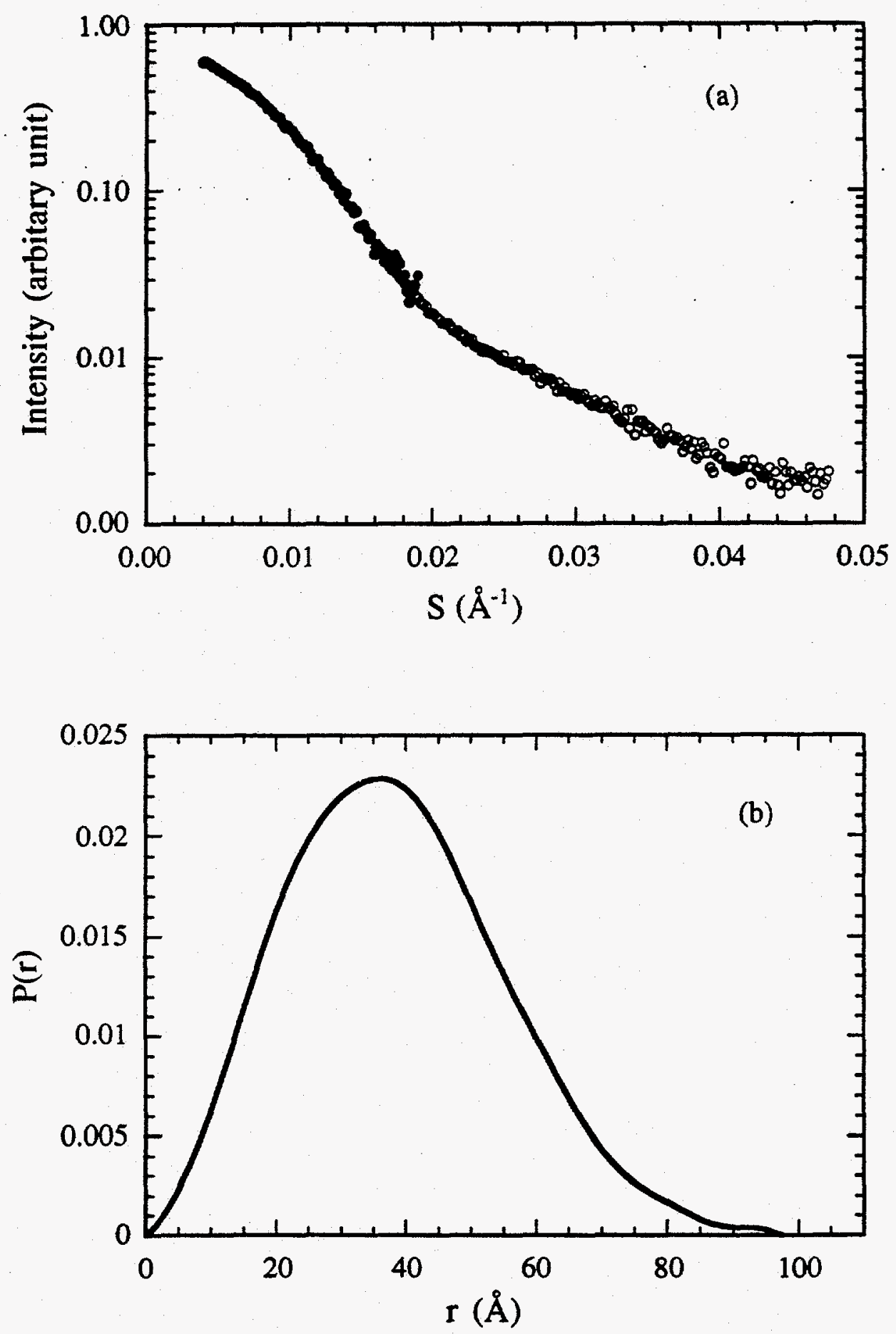

Figure 3.4. a) A combined scattering curve of BSA with the small angle range from the linear detector data $(\bullet)$, and the wide angle region from the quadrant detector $(O)$. The linear detector data is from the $4.7 \mathrm{mg} / \mathrm{ml}$ data set, and the quadrant detector data is from the $18.8 \mathrm{mg} / \mathrm{ml}$ BSA data set. $\mathrm{b})$ The $\mathrm{P}(\mathrm{r})$ function of this combined data. 

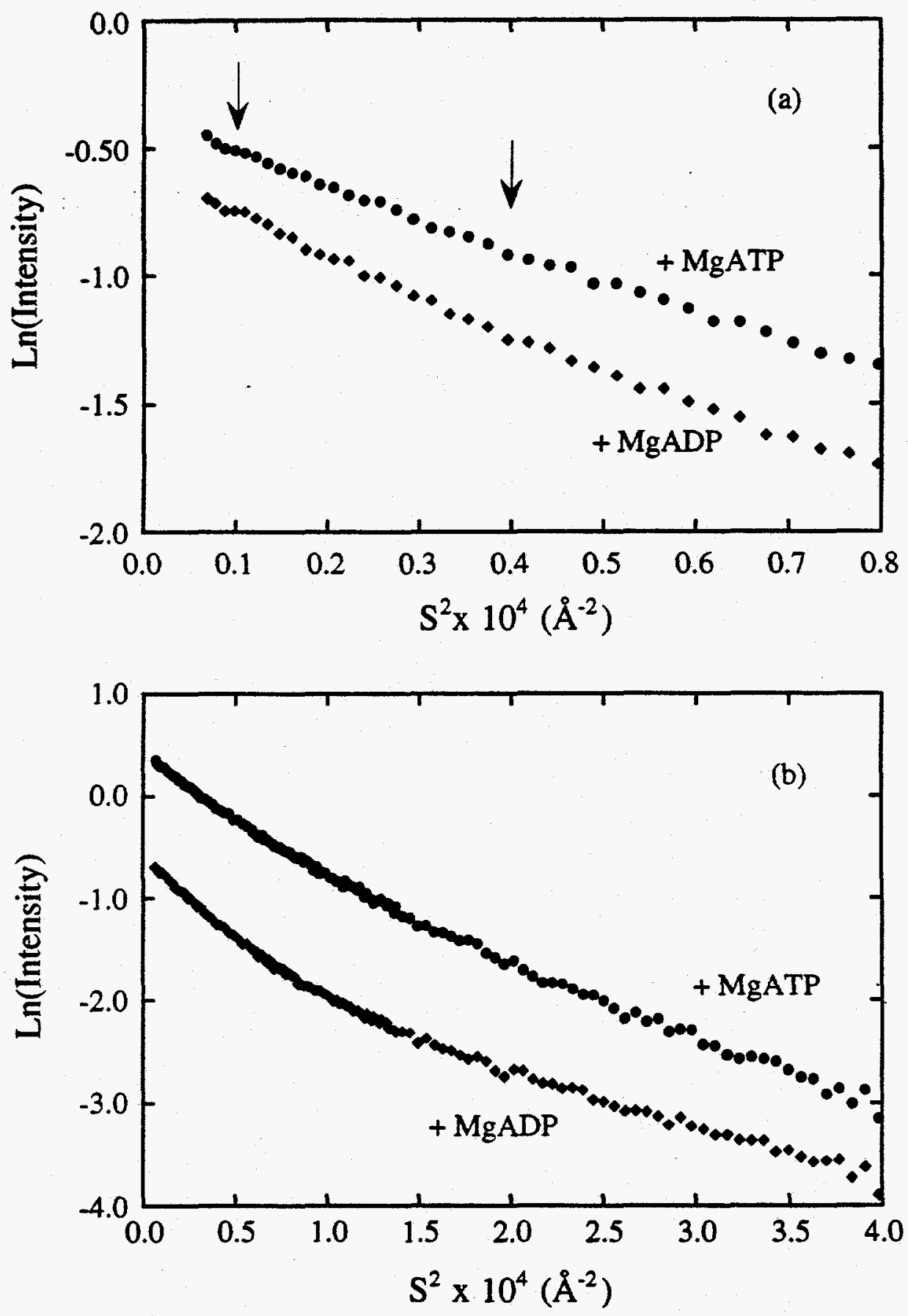

Figure 3.5. Representative scattering curves, in $\ln (\mathrm{I}(\mathrm{S}))$ versus $\mathrm{S}^{2}$ for the $60 \mathrm{kDa}$ fragment in the presence of $\operatorname{MgATP}(\bullet)$ and $\operatorname{MgADP}(\bullet)$. (a) at low angle, and (b) an extended region. Arrows in panel a shows region used to compute $R_{g}$ in the Guinier approximation. For clarity, the scattering curve collected with MgATP is shifted vertically from that collected with $\mathrm{MgADP}$ in both figures. 


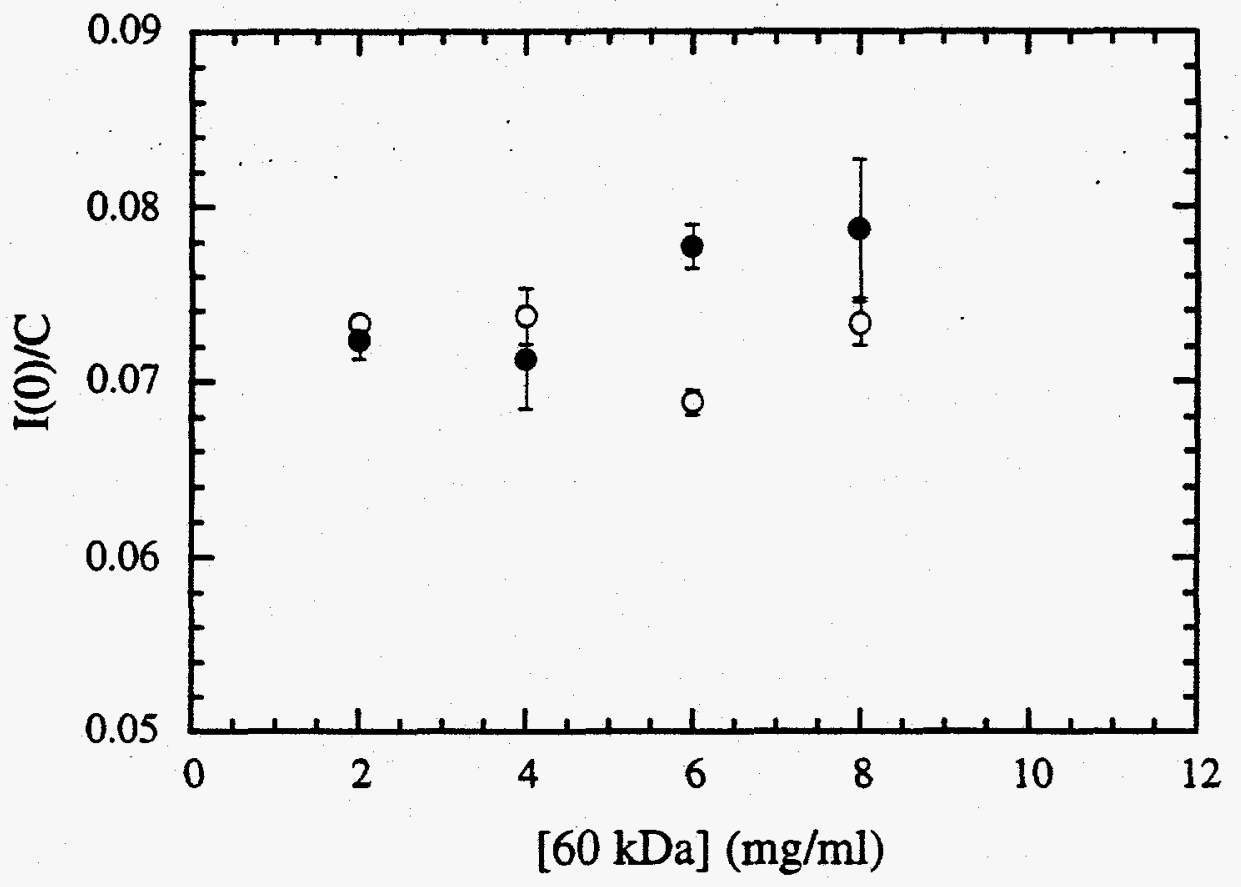

Figure 3.6. Forwarded scattering intensity normalized to protein concentration, $\mathrm{I}(0) / \mathrm{C}$, for the $60 \mathrm{kDa}$ fragment in the presence of $(\bullet) \mathrm{MgATP}$ and (O)MgADP. 
Reproducibility of data. In order to assess the combined reproducibility of the sample preparation protocols and the experimental apparatus, complete datasets were taken on two separate runs for each of the protein constructs (Table 3.1). Pairs of $R_{g}$ values determined on the same construct on different runs generally agreed within computed error (i.e. the standard deviation of the intercept value of a weighted linear fit to experimental values of $R_{\mathrm{g}}$ versus concentration); for example, the two values of $R_{\mathrm{g}}$ for the ATPase domain in the presence of MgADP differed by only $0.3 \pm 0.3 \AA$. The greatest variation was observed with hsc70 in the presence of ADP, where differences between values from these two independent determinations of $R_{g}$ were $2.6 \pm 1.2 \AA$ in the Guinier approximation, or $3.2 \pm 0.7 \AA$ by $\mathrm{P}(\mathrm{r})$ function analysis. This correlates with the fact that hsc70 shows the greatest propensity to aggregate in solution, and presumably reflects heterogeneity at the biochemical level, since variations of this magnitude did not occur with other samples. Because of this, an emphasis has been placed in the following discussion on using results from the constructs which are least variable in their behavior (ATPase and $60 \mathrm{kDa}$ fragments) for quantitative modeling, and then extending these results to hsc70 as far as is supported by the data.

Steady-state radii of gyration. Fig. 3.7 shows the concentration dependence of $R_{g}$ and the extrapolations to zero protein concentration. The extrapolation results, as well as results from $P(r)$ function analysis (see Section 3.4.1.3), are summarized in Table 3.1.

Scattering data were collected on samples of hsc70 in the concentration range 1-4 $\mathrm{mg} / \mathrm{ml}(14-57 \mu \mathrm{M})$ in the presence of $0.5 \mathrm{mM} \mathrm{MgATP}$ or MgADP. For MgATP, this concentration is well above the $\mathrm{K}_{\mathrm{m}}$ of steady-state hydrolysis $(\sim 0.1 \mu \mathrm{M})$, and the nucleotide:protein ratio is such that less than $5 \%$ of the ATP will be hydrolyzed during the course of the measurements. For MgADP, this concentration is well above the $\mathrm{K}_{d}$ of the nucleotide $(0.11 \mu \mathrm{M})$. In the presence of either MgATP or MgADP, the apparent $R_{g}$ increases slightly with increasing protein concentration (Fig. 3.7.a), suggesting that a small amount of aggregation may be present at the higher protein concentrations.

The $\mathrm{R}_{\mathrm{g}}$ measured in the presence of $\mathrm{MgATP}$ is substantially smaller than that measured in the presence of MgADP at all concentrations (Fig. 3.7.a and Table 3.1). Extrapolation of $R_{g}$ to zero protein concentration gives values of $-36 \AA$ in the presence of MgADP and $-33 \AA$ in the presence of saturating amounts of MgATP, suggesting that MgATP induces a significant conformational change in hsc70, relative to its 
Table 3.1. Steady-state structure parameters $(\AA)$ derived from solution scattering data for hsc70, the $60 \mathrm{kDa}$ fragment, and the 44 $\mathrm{kDa}$ ATPase domain in the presence of MgADP and MgATP and the peptide-binding fragment.

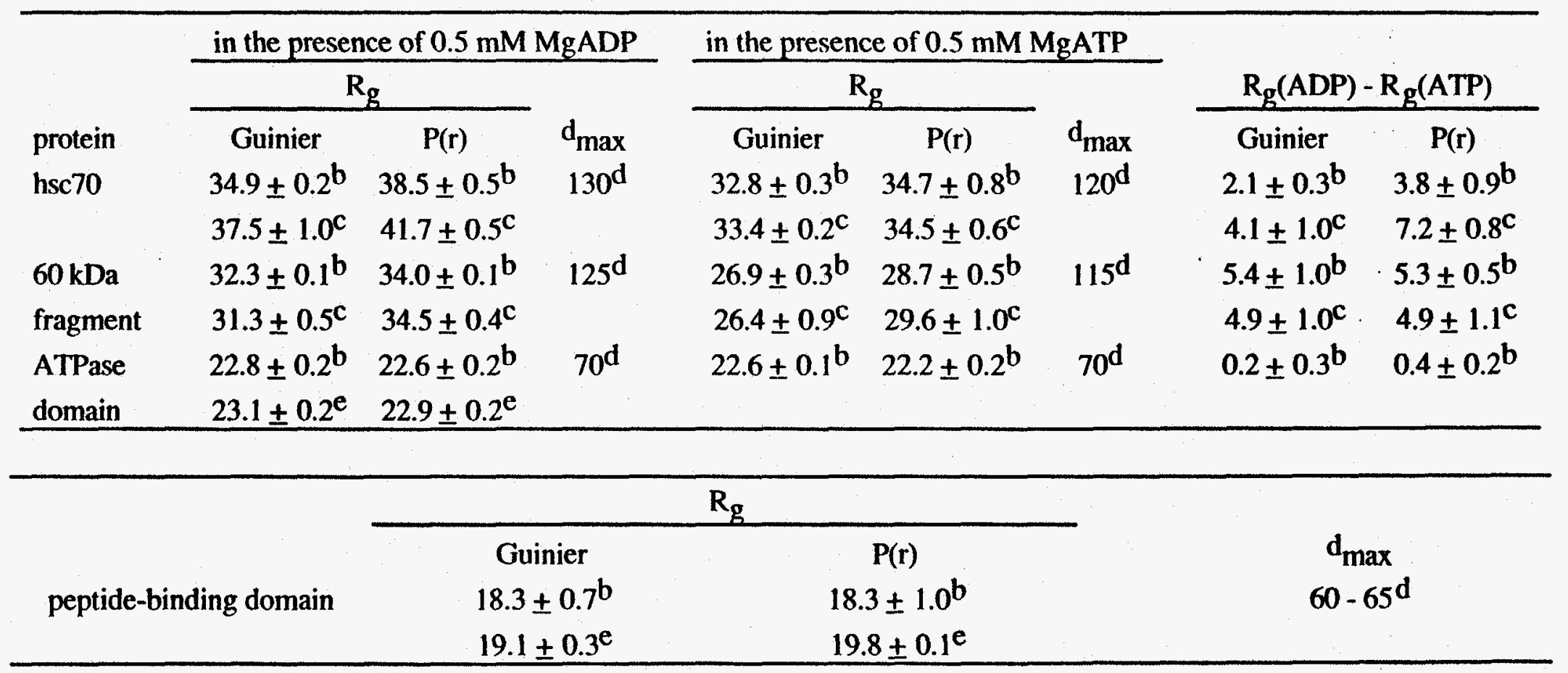

a Values for $\mathrm{Rg}$ are extrapolations to zero protein concentration from values determined at several concentrations, either by Guinier analysis or from $P(r)$ functions. $b$ Data derived from March 1994 run. ${ }^{c}$ Data derived from June 1994 run. $d$ Derived . from combined data of linear and quadrant detector, June 1994 run. ${ }^{e}$ Data derived from March 1995 run. 
Figure 3.7. The concentration dependence of steady-state radii of gyration computed by Guinier approximation for (a) Hsc70; (b) the $60 \mathrm{kDa}$ fragment; (c) ATPase fragment; (d) the peptide binding domain. For a-c, $(\bullet)$, in the presence of $\operatorname{MgADP} ;(\bullet)$, in the presence of MgATP; $(\mathrm{O})$ in the presence of MgATP with $\mathrm{Na}^{+}$rather than $\mathrm{K}^{+}$. Multiple measurements were taken at every concentration. Errors associated with individual measurement were derived from the least squares fitting of the data in the Guinier regions. The error bar shown at each concentration in the figures is chosen to be the larger value between the maximum of individual errors and the standard deviation from the mean value of different measurements. Solid and dashed lines represent the extrapolation to zero protein concentration in the presence of MgATP and MgADP, respectively. 

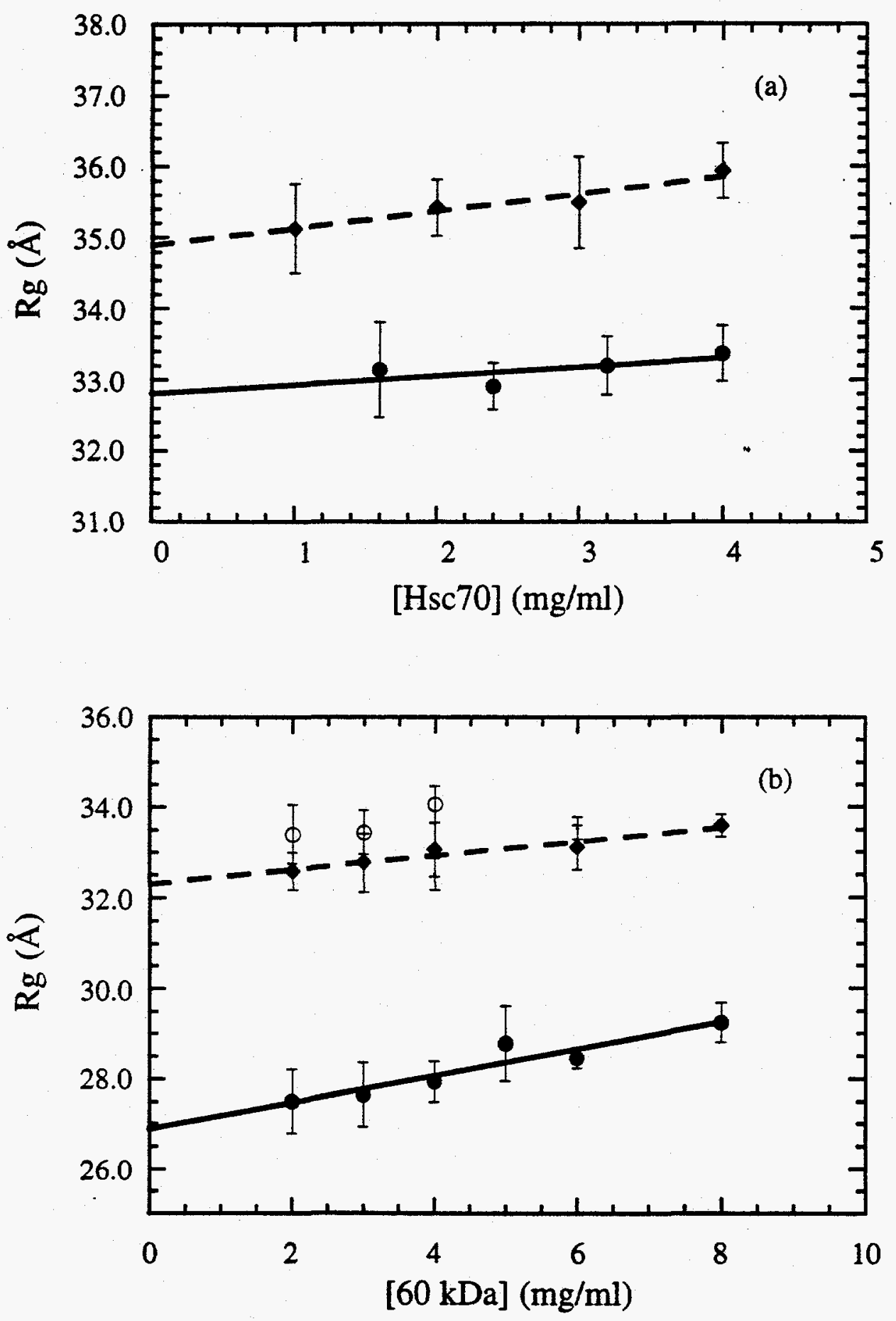

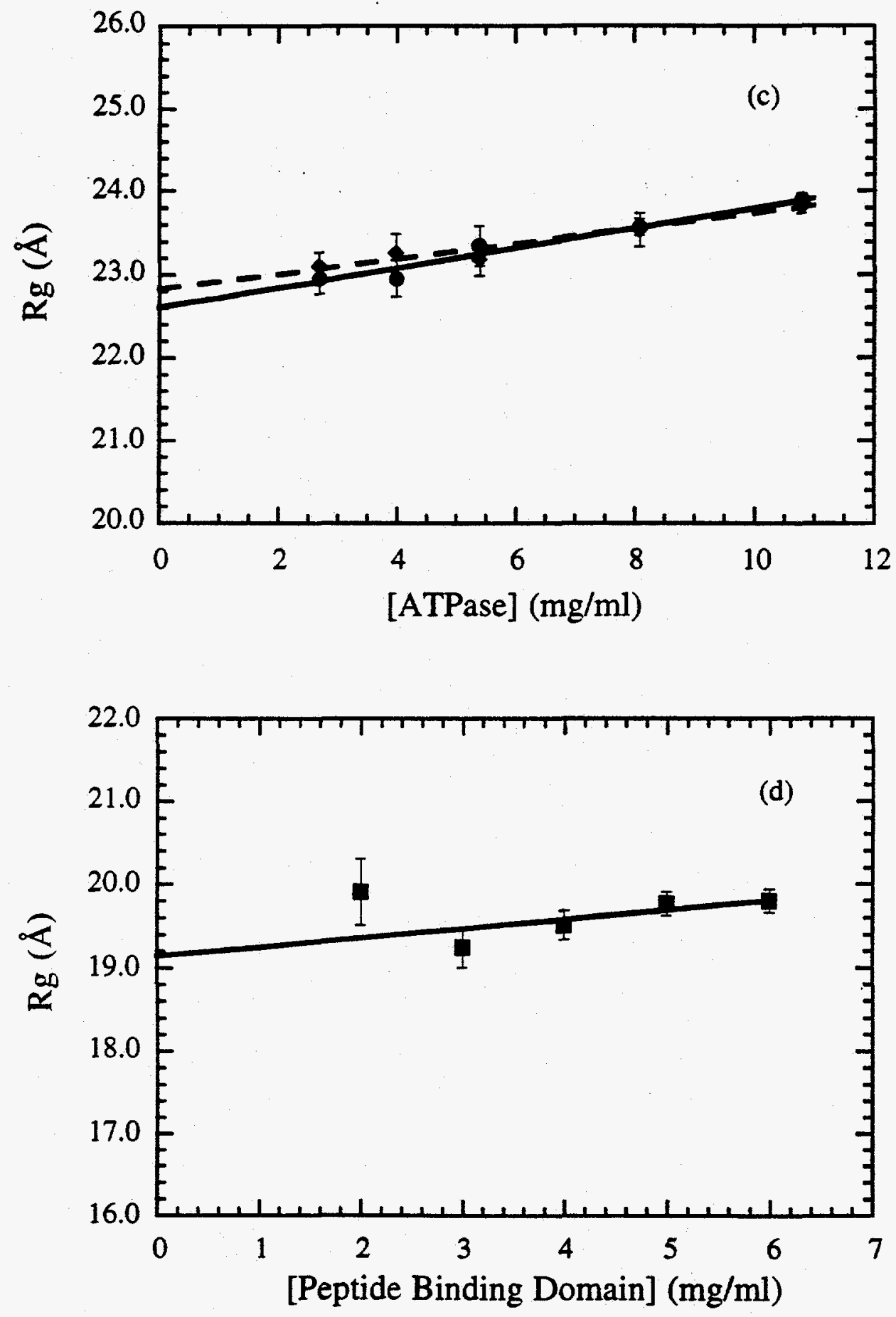
conformation in the presence of MgADP. The concentration-normalized values of $\mathrm{I}(0)$ are the same in the presence of either nucleotide and are consistent with a molecular weight of $\sim 70 \mathrm{kDa}$. Thus, under both conditions hsc70 is largely monomeric; the difference in $\mathrm{R}_{\mathrm{g}}$ between that observed with MgATP and that observed with MgADP is not due to oligomerization.

Scattering curves were also recorded for hsc70 at $4 \mathrm{mg} / \mathrm{ml}$ in absence of nucleotide (Table 3.2). The computed $\mathrm{R}_{\mathrm{g}}$ shows no statistically significant difference from that with MgADP present. Data were also collected in the presence of $1 \mathrm{mM}$ MgAMPPNP, a slowly hydrolyzable analog of ATP. Again, the computed $\mathbf{R}_{\mathbf{g}}$ is equal within experimental error to that observed with $\mathrm{MgADP}$ present. Only MgATP was able to induce the conformational change to the smaller $\mathrm{R}_{\mathrm{g}}$ in hsc70.

To test whether the $60 \mathrm{kDa}$ fragment, which does not contain the -100 amino acid residues at the carboxyl terminal of hsc70, undergoes a conformational change similar to that observed in hsc70, data were collected and analyzed in a similar manner from samples containing 1-8 $\mathrm{mg} / \mathrm{ml}$ protein (Fig. 3.7.b). The MgATP-containing sample yields a substantially smaller $R_{g}$ than the sample with MgADP (Table 3.1). Further, scattering curves were measured and $\mathrm{R}_{\mathrm{g}} \mathrm{s}$ were computed for several concentrations of $60 \mathrm{kDa}$ fragment in the absence of nucleotide, in the presence of slowly-hydrolyzable ATP analogs AMPPNP and AMPPCP, and in the presence of MgATP but with $\mathrm{Na}^{+}$replacing $\mathrm{K}^{+}$in the sample buffer; representative values of $R_{\mathrm{g}}$ determined at $4.0 \mathrm{mg} / \mathrm{ml}$ protein concentration are summarized in Table 3.2. In all cases, the apparent $R_{g}$ agreed within error to that observed with MgADP present. Thus, the transition to the smaller $\mathrm{R}_{\mathrm{g}}$ requires MgATP (slowly hydrolyzable analogs can not substitute) and $\mathrm{K}^{+}\left(\mathrm{Na}^{+}\right.$can not substitute). Since the $60 \mathrm{kDa}$ fragment shows an ATP-induced conformational change similar to that of hsc70 and has less tendency to aggregate, many of the subsequent experiments were conducted on it in parallel with experiments on hsc70.

Both hsc70 and the $60 \mathrm{kDa}$ fragment, particularly in the presence of MgADP and slowly hydrolyzable analogs and in the absence of nucleotides, do not appear to be globular in shape. Using $1.21 \AA^{3}$ /dalton as partial specific volume, $R_{g}$ of hsc70 and the $60 \mathrm{kDa}$ fragment would be $-21 \AA$ and $-20 \AA$ if both were spherical. Even with a factor of $40 \%$ increase in volume to account for a hydration layer (Section 3.4.3.1) around the protein molecule in solution, $R_{g}$ would only be increased to $\sim 24 \AA$ and $\sim 22 \AA$ for spherical hsc70 and the $60 \mathrm{kDa}$ fragment. A more direct and sensible approach is to 
Table 3.2. Representative $R_{g}$ values ( $\AA$; computed in Guinier approximation) for hsc70, the $60 \mathrm{kDa}$ fragment, and the $44 \mathrm{kDa}$ ATPase fragment under different nucleotide conditions ${ }^{\mathrm{a}}$.

\begin{tabular}{|c|c|c|c|c|c|c|}
\hline \multirow[b]{2}{*}{ protein } & \multicolumn{3}{|c|}{ nucleotides } & \multirow[b]{2}{*}{ no nucleotide } & \multicolumn{2}{|c|}{ MgATP } \\
\hline & MgADP & MgAMPPNP & MgAMPPCP & & $\mathrm{Na}^{+}$buffer & $\mathrm{K}^{+}$buffer \\
\hline hsc70 & $35.5 \pm 0.6^{b}$ & $36.8 \pm 0.3^{b}$ & N.D. & $37.4 \pm 0.4^{b}$ & N.D. & $33.4 \pm 0.4^{b}$ \\
\hline $60 \mathrm{kDa}$ fragment & $33.1 \pm 0.6^{b}$ & $32.5 \pm 0.3^{b}$ & $32.7 \pm 0.3^{c}$ & $32.6 \pm 0.5^{c}$ & $34.1 \pm 0.4^{c}$ & $27.9 \pm 0.5^{b}$ \\
\hline ATPase domain & $\begin{array}{l}22.7 \pm 0.2^{b} \\
23.1 \pm 0.1^{d}\end{array}$ & N.D. & N.D. & $23.8 \pm 0.1^{d}$ & N.D. & $22.5 \pm 0.2^{b}$ \\
\hline
\end{tabular}

a Values for hsc70 and the $60 \mathrm{kDa}$ fragment were determined at a single protein concentration, usually $4 \mathrm{mg} / \mathrm{ml}$, except for the 60 $\mathrm{kDa}$ fragment with MgAMPPNP and without nucleotides, where the concentration was $3 \mathrm{mg} / \mathrm{ml}$. Values for $\mathrm{R}_{\mathrm{g}}$ of the ATPase fragment are extrapolations to zero concentration. ${ }^{b}$ Data derived from March 1994 run. ${ }^{c}$ Data derived from June 1994 run. $d$ Data derived from March 1995 run. Values not determined are labeled N.D. 
compare $R_{\mathrm{g}}$ with a known globular-shaped protein molecule of a similar molecular weight. BSA has a MW of $66 \mathrm{kDa}$, between the $60 \mathrm{kDa}$ fragment (MW $-60 \mathrm{kDa}$ ) and hsc70 (MW $-72 \mathrm{kDa}$ ). Compared with the $\mathrm{R}_{\mathrm{g}}(29.6 \pm 0.2 \AA$ ) of BSA (Section 3.4.1.1), the even smaller $\mathrm{MW}$ for the $60 \mathrm{kDa}$ fragment appears to be rather elongated both in the presence of $\mathrm{MgADP}$ or slowly hydrolyzable analogs and in the absence of nucleotides, all having $R_{g}$ of $\sim 33 \AA$ (Table 3.2). A similar conclusion is also reached for hsc70. More about shape analysis of the $60 \mathrm{kDa}$ fragment and hsc70 will be discussed in Section 3.4.1.3.

To test whether the change in $R_{\mathrm{g}}$, detected in both the $60 \mathrm{kDa}$ fragment and hsc70, could be localized to the nucleotide binding domain of hsc70, data were collected and analyzed in the same way from samples containing $1-11 \mathrm{mg} / \mathrm{ml}$ ATPase domain (Fig. 3.7.c). Extrapolation of $R_{g}$ to zero concentration yields values of $22.8( \pm 0.2) \AA$ in the presence of MgADP and $22.6( \pm 0.2) \AA$ in the presence of MgATP, revealing no detectable difference in conformation between the two states in solution. This is consistent with crystallographic results on wild-type and several mutant proteins which show the ATPase domain to have the same overall conformation in the presence of MgATP or MgADP (Flaherty, et al. , 1995).

An increase of at least $0.7 \pm 0.2 \AA$ in $R_{g}$ from the Guinier analysis was observed for the ATPase domain in the absence of nucleotides compared with those in the presence of MgADP (and MgATP) (Table 3.2). A somewhat large $\mathrm{R}_{\mathrm{g}}$ in the absence of nucleotide suggests that a "hexokinase-like" opening of the nucleotide binding cleft may occur upon release of nucleotide (Bennett \& Steitz, 1980; Fletterick, et al., 1975). An increment in the $\mathrm{R}_{\mathrm{g}}$ of the $60 \mathrm{kDa}$ fragment in absence of nucleotide was not observed; this is not unexpected, since (if the center-to-center distance of the ATPase and peptide binding domains remained essentially unchanged when nucleotide was released) only a $0.3 \AA$ increase in $R_{g}$ would be expected for this fragment for a conformational change that increased $R_{g}$ of the ATPase fragment by $0.7 \AA$.

Scattering curves were also collected for the peptide binding domain (Fig. 3.7.d). Data from two different runs (June 1994 and March 1995) yielded $R_{\mathbf{g}}$ values $\sim 19 \AA$. 


\subsubsection{The $P(r)$ Function Analysis on hsc70 and Its Subfragments}

Compar ison with the Guinier analysis. A quadrant detector was employed in the data collection to improve statistics at wide angular range for $\mathrm{P}(\mathrm{r})$ function analysis. Radii of gyration were also evaluated from $P(r)$ functions via equation [3], and are compared with those from the Guinier analysis in Table 3.1. For the smaller protein constructs (ATPase and peptide binding fragments), the maximum values of $2 \pi S R_{g}$ used in the Guinier analysis ranged 1.1-1.3, and the $R_{\mathrm{g}}$ values computed (i) using the Guinier approximation and (ii) using the $P(r)$ function agree within experimental error (Table 3.1). For the larger proteins (hsc70 and the $60 \mathrm{kDa}$ fragment), $R_{g}$ s computed from $P(r)$ functions were systematically $\sim 2 \AA$ larger than those computed using the Guinier analysis, and maximum values of $2 \pi S R_{g}$ used in the Guinier analysis ranged 1.1-1.5. For elongated molecules, such as the $60 \mathrm{kDa}$ fragment and hsc70 in this study, the constraint on the range of $S$ in the Guinier fitting $\left(2 \pi \mathrm{SR}_{\mathrm{g}} \ll 1.0\right)$ becomes more stringent. The disagreement between $\mathrm{R}_{\mathrm{g}} \mathrm{s}$ calculated by the two methods may be due to the use of a relatively generous Guinier region for the larger proteins, which was necessitated by the fact that restricting the analysis to smaller scattering angles reduced the number of data points included in the linear fit to fewer than 12 in some cases, at which point the errors in the detector response correction of individual channels significantly affected the calculated slope of the linear fit used for the Guinier analysis.

As discussed in Section 3.4.1.2, the largest discrepancy in data reproducibility was observed with hsc70 in the presence of MgADP, and the variation can be correlated with the fact that hsc70 shows greatest tendency to aggregate in solution (more about this will be discussed in Section 3.5.). Allowing for the variability of results on hsc70 in the presence of $A D P$, values of the change of $R_{g}\left(\Delta R_{g}\right.$ ) between different nucleotide (e.g. with $\mathrm{MgATP}$ versus with $\mathrm{MgADP}$ ) conditions computed by the two different analyses (Guinier and $\mathrm{P}(\mathrm{r})$ analysis) agree within statistical error. Consequently, it appears that the $\Delta R_{g}$ values determined from the Guinier analyses of small angle data are as reliable as those computed by incorporating higher angle data into the $\mathrm{P}(\mathrm{r})$ function. In this study, Guinier values of $R_{g}$ are used to parameterize the magnitudes and kinetics of nucleotideinduced conformational changes, as revealed by changes in $R_{\mathrm{g}}$. The validity of using the $P(r)$ function analysis of scattering data to higher angle has been established in the study of BSA (Section 3.4.1.1) where consistent $R_{\mathbf{g}} \mathbf{s}$ derived from the Guinier approximation and from $P(r)$ analysis are obtained. In addition, comparisons are made below (Section 3.4.3) between experimental $P(r)$ functions for the ATPase fragment and those computed 
from crystallographic coordinates; the agreement between the measured and computed distributions further supports the assertion that values of $R_{g}$ derived from the $P(r)$ function is accurate.

Calculation of $\mathbf{P ( r )}$ functions. To acquire more information on the overall shape of hsc70 and its subfragments, we collected data from each construct at $4 \mathrm{mg} / \mathrm{ml}$ with both the linear detector (better resolution at low angle) and the quadrant detector (greater signal at higher angle) and used the combined scattering curves to calculate $P(r)$ functions. (The validity of combining data from linear detector and quadrant detector for $P(r)$ function calculation has been discussed in Section 3.4.1.1) In addition, $P(r)$ functions were calculated from data collected solely on the linear detector, including data outside the Guinier region, for samples at concentrations other than $4 \mathrm{mg} / \mathrm{ml}$. $P(r)$ functions computed using data from only the linear detector showed no significant differences, but associated with larger error bars, from those computed using combined data to higher angle from the linear and quadrant detectors.

The $\mathrm{P}(\mathrm{r})$ functions for the $60 \mathrm{kDa}$ fragment and hsc70 (Fig. 3.8) share several features which are consistent with elongated proteins that undergo substantial conformational changes. First, the $d_{\max }$ values (120-130 $\AA$ for hsc70; $115-125 \AA$ for the $60 \mathrm{kDa}$ fragment) indicate that the molecules are elongated. As a comparison, BSA, a globular shape protein with similar $M W$, has a smaller $d_{\max },-95 \AA$. Second, the $P(r)$ functions for both proteins in the presence of MgADP show distinct shoulders at 70-80 $\AA$, suggestive of a bilobal shape. Again, the shape of the $P(r)$ functions contrasts with a symmetric singular maximum $P(r)$ function of a globular shape BSA (Fig. 3.4.b). Third, the $P(r)$ functions for the proteins in the presence of MgATP reflect a substantial contraction relative to that for MgADP; the shoulders are much diminished and the $d_{\max }$ values are substantially smaller (Table 3.1 ).

The $P(r)$ functions were also computed for the ATPase domain and the peptidebinding domain (Fig. 3.9), and the derived parameters are listed in Table 3.1. More about the $P(r)$ functions of these two constructs will be discussed in the modeling section, Section 3.4.3. 

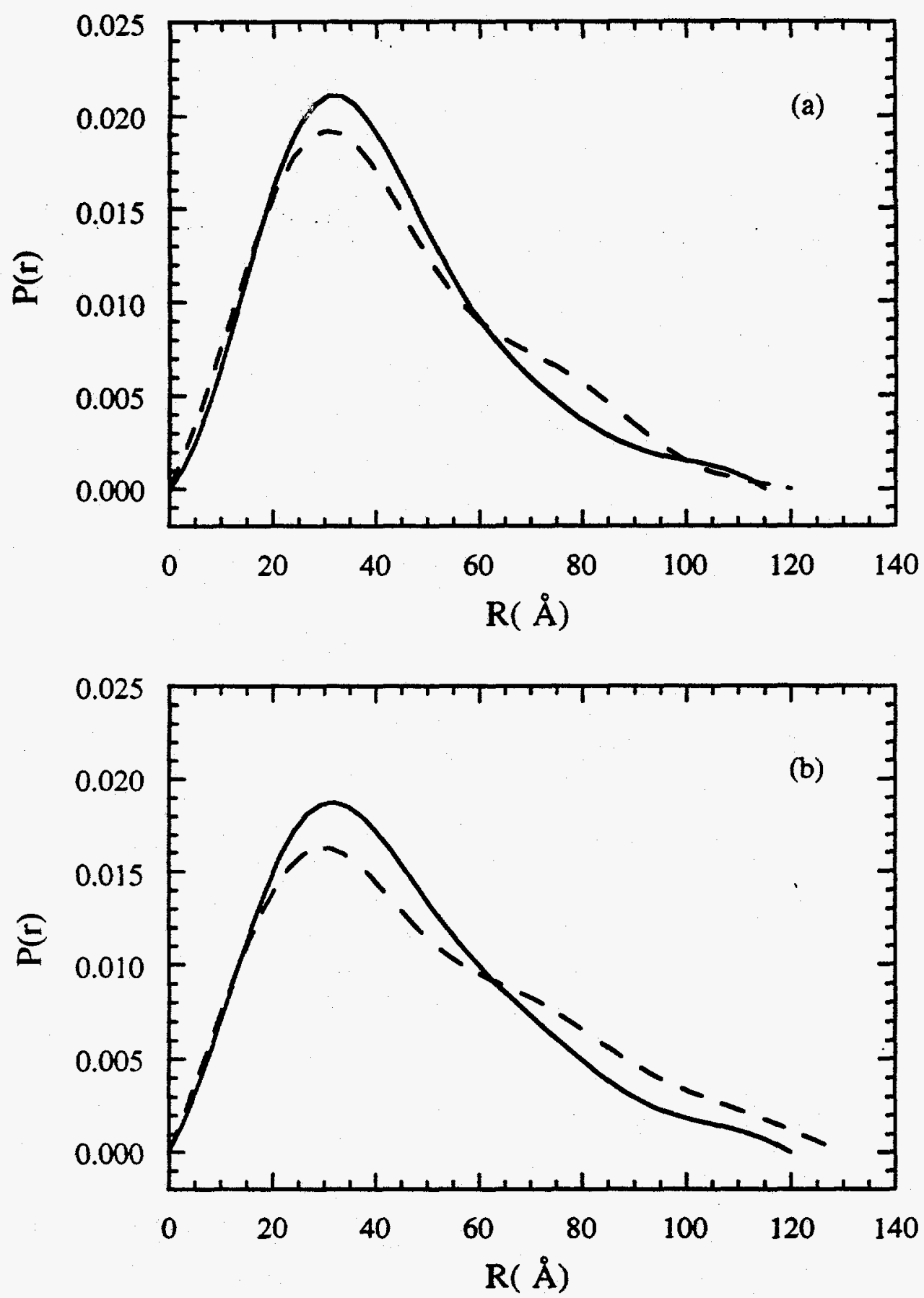

Figure 3.8. Distance distribution functions for (a) the $60 \mathrm{kDa}$ fragment; (b) the fulllength hsc70, in the presence of $\operatorname{MgATP}(-)$, and in the presence of MgADP (---). 


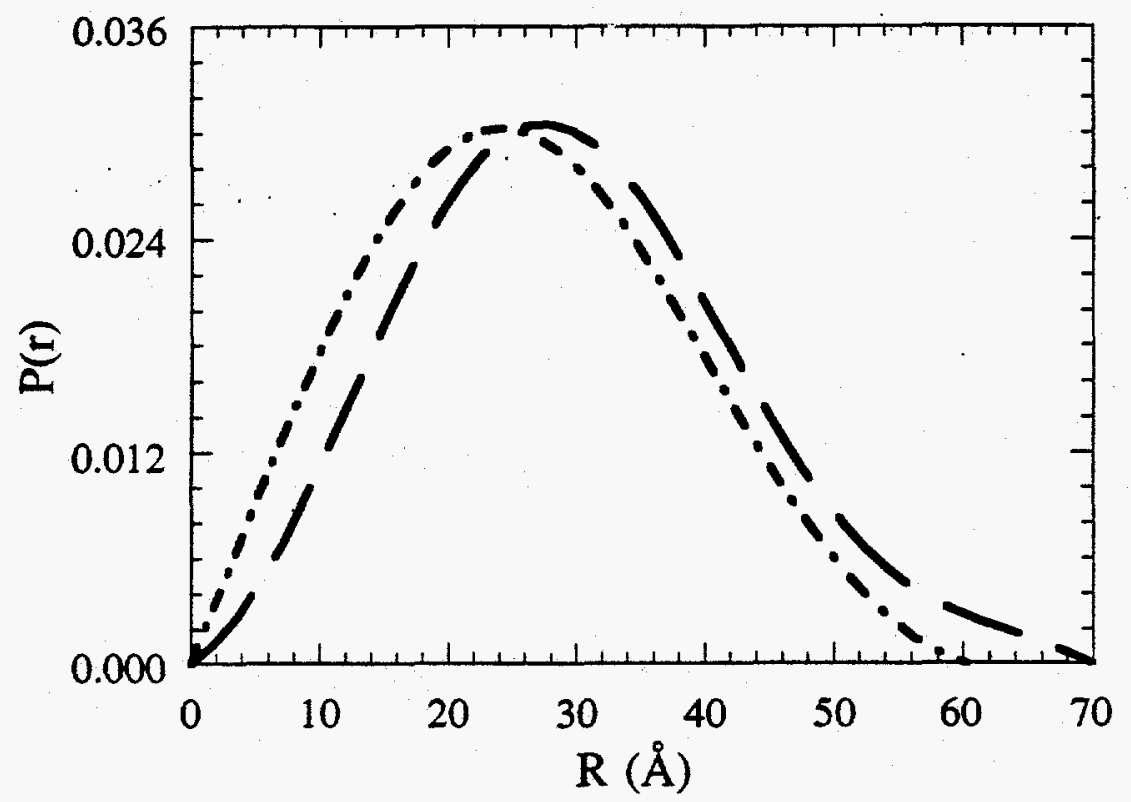

Figure 3.9. Distance distribution functions for the ATPase domain (- - ), and the peptide binding domain (-- -). 


\subsubsection{Kinetic Measurements}

Kinetics of the ATPase cycle of the $60 \mathrm{kDa}$ fragment. The rate of ATP hydrolysis was measured for the $60 \mathrm{kDa}$ fragment under single turnover conditions, using experimental protocols and data analysis methods described previously (Ha \& McKay, 1994). Fit of a single exponential to data for the fraction of ATP hydrolyzed versus time yielded a rate of $0.0017 \pm 0.0001 \mathrm{sec}^{-1}\left(t_{1 / 2}=7 \mathrm{~min}\right.$., Fig. 3.10.a). When protein is mixed with stoichiometric amounts of $\left.\psi^{32} \mathrm{P}\right]-\mathrm{ATP}$ and the release of inorganic phosphate after binding and hydrolysis of the nucleotide was followed, it did not differ significantly from the rate of hydrolysis, demonstrating that phosphate release occurs rapidly after, and is significantly faster than, hydrolysis. The rate of dissociation of ADP from complexes of the $60 \mathrm{kDa}$ fragment pre-incubated with $\alpha$-32P-ATP for a sufficiently long time to allow complete hydrolysis was measured to be $>0.008 \mathrm{sec}^{-1}$. The steady state ATPase rate was measured to be $0.0024 \pm 0.0002 \mathrm{sec}^{-1}$. These data show that under the conditions of our experiments $\left(\mathrm{T}=25^{\circ} \mathrm{C}\right)$, ATP hydrolysis is the rate-limiting step of the ATPase cycle for the $60 \mathrm{kDa}$ fragment. The impossibly low hydrolysis rate (in light of the steady state turnover rate) probably reflects experimental error and gives an estimate of the size of that error. The kinetic study was performed by Dr. Sigurd Wilbanks at Prof. McKay's laboratory of Department of Structural Biology, Stanford Medical School.

Kinetics of the change in $\mathbf{R}_{\mathbf{g}}$. To determine the time course of the change in $\mathbf{R}_{\mathbf{g}}$, data were collected for both hsc70 (March 1995) and the $60 \mathrm{kDa}$ fragment (June 1994) after manual mixing of nucleotide-free protein with sub-stoichiometric amounts of MgATP (80-90\%). Because radiation damage precluded data collection for longer than 10 minutes (for the $60 \mathrm{kDa}$ fragment, June 1994) or 20 minutes (for hsc70, March 1995) from a single sample, longer time courses were assembled by transferring an aliquot from a master reaction to the sample cell every 12 minutes (the $60 \mathrm{kDa}$ fragment) or 22 minutes (hsc70). The temperature of both cell and master reaction were maintained at $25^{\circ} \mathrm{C}$. $R_{\mathrm{g}}$ values were computed for each one-minute or two-minute accumulation of data (Fig. 3.10). For both proteins, the minimum value of $\mathbf{R}_{\mathbf{g}}$ had been reached at the earliest data collection time ( $\sim 1.5$ minutes). The minimum $R_{g}$ is equal to the steady state value measured in the presence of excess MgATP. The observed $R_{g}$ returned to the larger value, equal to (or larger than) that observed with excess MgADP in steady state measurements over the period of minutes. 

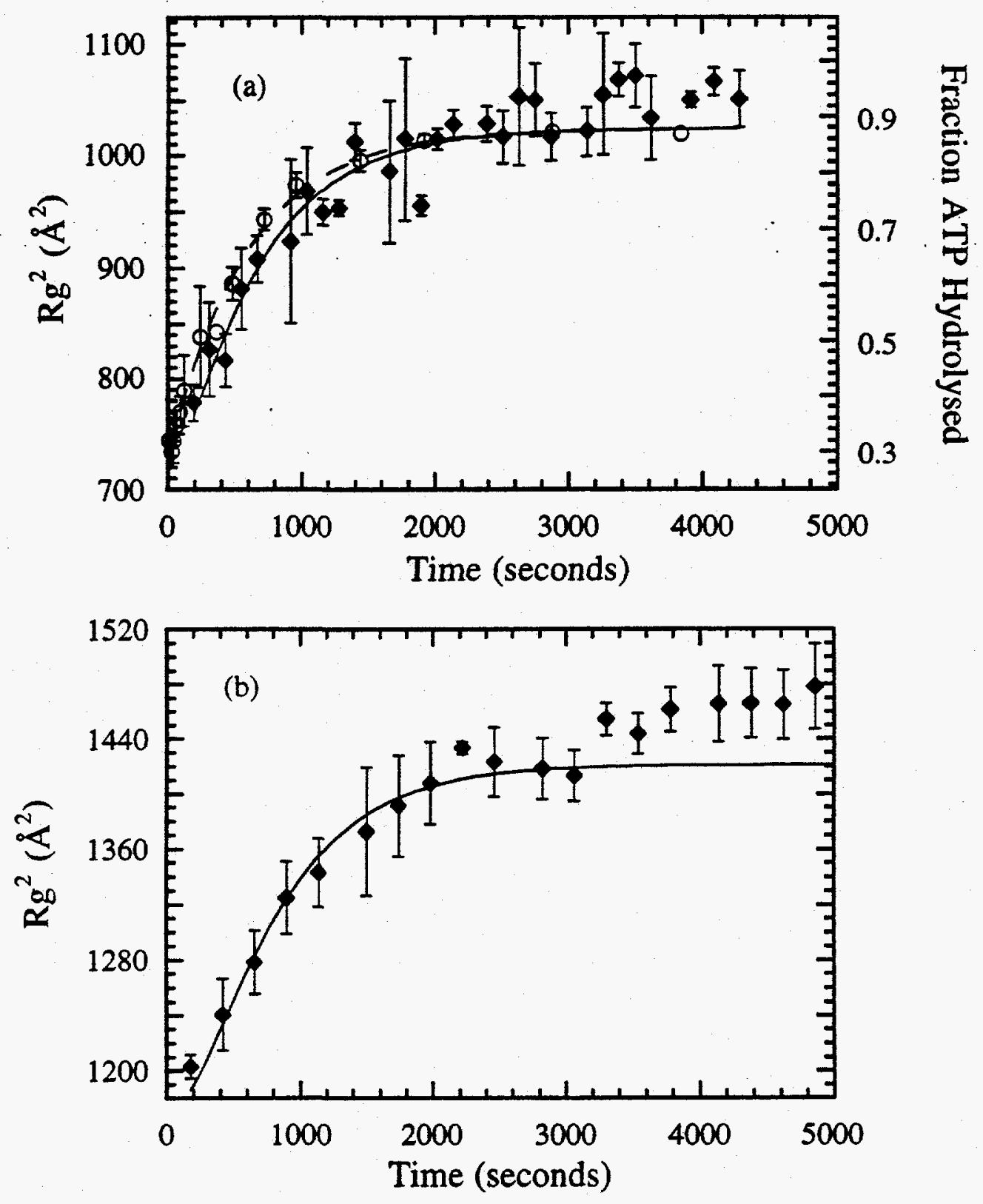

Figure 3.10. Time dependence of $R_{\mathrm{g}}^{2}$ after mixing $80 \mu \mathrm{M}$ protein, $65-75 \mu \mathrm{M}$ ATP at 25 ${ }^{\circ} \mathrm{C}$. $\mathrm{R}_{\mathrm{g}}^{2}$, computed in Guinier approximation, is shown as $(\bullet)$; each time point represents the average of three separate experiments. (a) $60 \mathrm{kDa}$ fragment. Solid curve shows result of double exponential fit to the data. ATP hydrolysis under single-turnover conditions is shown in (O) with a single exponential fit (dashed curve). (b) Hsc70. Solid curve shows results of double exponential fit to the data, with initial and final values of the curve were constrained to be equal to steady state values of $R_{g}$ in presence of ATP and ADP respectively, and with first rate constant equal to that of ATP hydrolysis. 
The values of $R_{g}^{2}$ as a function of time were analyzed as a linear combination of the steady-state values of $R_{g}^{2}$ in the presence of MgADP and the presence of MgATP. For hsc70, estimating the rate of return to the larger $R_{g}^{2}$ by fitting a single exponential to the data gave a rate constant of $0.0012 \pm 0.0001 \mathrm{sec}^{-1}\left(\mathrm{t}_{1 / 2}=9.6 \mathrm{~min}\right.$.). Under the conditions of these experiments $\left(\mathrm{T}=25^{\circ} \mathrm{C}\right)$, the rate constant for ATP hydrolysis is $0.0030 \mathrm{sec}^{-1}\left(\mathrm{t}_{1 / 2}=3.8 \mathrm{~min}\right.$.); the relaxation to the large $\mathrm{R}_{\mathrm{g}}^{2}$ is significantly slower than ATP hydrolysis. Therefore, the return of $R_{g}^{2}$ to the larger value was simulated as a twostep process, with the first step having the rate constant $\left(\mathrm{k}_{\mathrm{hyd}}\right)$ of hydrolysis:

$$
R_{g}^{2}(\text { apparent })=R_{g}^{2}(\text { steady - stateADP })-\Delta\left(R_{g}^{2}\right) \frac{k_{h y d} e^{-k_{\beta} t}-k_{\beta} e^{-k_{h y d} t}}{k_{h y d}-k_{\beta}}
$$

where $R_{g}^{2}$ (steady state ADP) and $\Delta R_{g}^{2}$ determined by steady state measurements under conditions identical (except for nucleotide concentration) to those of the kinetic experiment (Fig. 3.10). This yields a value $0.0019 \pm 0.0003\left(t_{1 / 2}=6.1 \mathrm{~min}\right.$.) for the second rate constant, $\mathrm{k}_{\beta}$.

Product release from hsc70 is sequential (Ha \& McKay, 1994); $P_{i}$ dissociates first with $\mathrm{k}_{\text {off }}\left(\mathrm{P}_{\mathrm{i}}\right)$ of $0.0038 \mathrm{sec}^{-1}\left(\mathrm{t}_{1 / 2}=3.0 \mathrm{~min}\right.$.); ADP then dissociates more rapidly with $k_{\text {off }}(A D P)$ of $0.029 \mathrm{sec}^{-1}\left(t_{1 / 2}=0.4 \mathrm{~min}\right.$.). The value of $k_{\beta}$ determined here is significantly slower than the rates of product release. The possibility that this difference may be due to prep-to-prep variation in the protein cannot be ruled out; nor can it be ruled . out that the difference is significant. Therefore, the return to the large $R_{g}$ form of hsc70 after binding of MgATP may coincide with, or may be a relaxation process predicated on, product release; it does not coincide with, and is too slow to be correlated with, ATP hydrolysis.

This conclusion is corroborated by the kinetics of the change of $R_{g}$ of the $60 \mathrm{kDa}$ fragment. The return to a large $R_{g}^{2}$ could be fit as an exponential decay with rate constant of $0.0009 \pm 0.0002 \mathrm{sec}^{-1}\left(\mathrm{t}_{1 / 2}=13 \mathrm{~min}\right.$.), to be compared with a rate of hydrolysis of $0.0017 \mathrm{sec}^{-1}$ (Fig. 3.10.a). If a two-step process is used to model the data, using the ATP hydrolysis rate constant for the first step, then the second step has a rate constant $0.0070 \pm 0.0020 \mathrm{sec}^{-1}\left(\mathrm{t}_{1 / 2}=1.6 \mathrm{~min}\right.$.). For the $60 \mathrm{kDa}$ fragment, release of both $\mathrm{P}_{\mathrm{j}}$ and $\mathrm{ADP}$ are rapid compared to hydrolysis, so that it is not possible to discriminate between 
hydrolysis and product release as concisely as with hsc70. However, it is clear that when a single ATPase cycle is initiated synchronously, the time required for the return to the larger $R_{\mathrm{g}}$ is not faster than the time required for hydrolysis and product release in the 60 $\mathrm{kDa}$ fragment.

At times longer than 50 minutes in the kinetic experiments, hsc70 reaches an $\mathrm{R}_{\mathrm{g}}$ larger than that measured at steady state. It is not known whether this is due to aggregation of the sample, adoption of a third, even more extended conformation or some other phenomenon.

\subsubsection{Modeling Based on $P(r)$ Functions}

\subsubsection{Modeling the ATPase and the Peptide-binding Domains}

$P(r)$ functions were used to construct a rudimentary model for hsc70 and its subfragments. Initially, correspondence was established between the $P(r)$ function derived from scattering data and $P(r)$ functions computed from crystallographic coordinates of the ATPase fragment. The crystallographic model of the ATPase fragment included amino acid residues 4-381, one ADP molecule, ore $\mathrm{P}_{\mathrm{i}}$, one $\mathrm{Mg}^{2+}$ ion, two $\mathrm{K}^{+}$ ions, one $\mathrm{Cl}^{-}$ion, and in some calculations, the $205 \mathrm{H}_{2} \mathrm{O}$ molecules that are within $3.0 \AA$ of protein, nucleotide, or cofactor atoms. The value of $\mathbf{R}_{\mathbf{g}}$ calculated from these coordinates, with or without the 205 water molecules, was $21.0 \AA$; the $P(r)$ function computed from the coordinates showed significant disagreement with the function derived from experimental scattering data. However, addition of an appropriate layer of hydration (an approximately $3 \AA$ shell with an electron density contrast of 0.03 electrons $/ \AA^{3}$ greater than that of solvent) to the crystallographic model, using the method of Svergun as implemented in the CRYSOL program package (Svergun, et al., 1995), yielded good agreement between both $R_{g}(22.5 \AA)$ and the overall $P(r)$ function (Fig. 3.11.a). We obtained a similar agreement between experimental and calculated $P(r)$ functions only when a layer of hydration was added to the crystallographic structure of the molecule for a second test case, lysozyme, using data collected during these experiments (see Chapter 4).

A rectangular prism (or box) was chosen as a simple (described by three parameters) and convenient geometric shape for modeling the ATPase domain. The $R_{g}$ of a box calculated with equation [9] was constrained to equal to the experimental value 

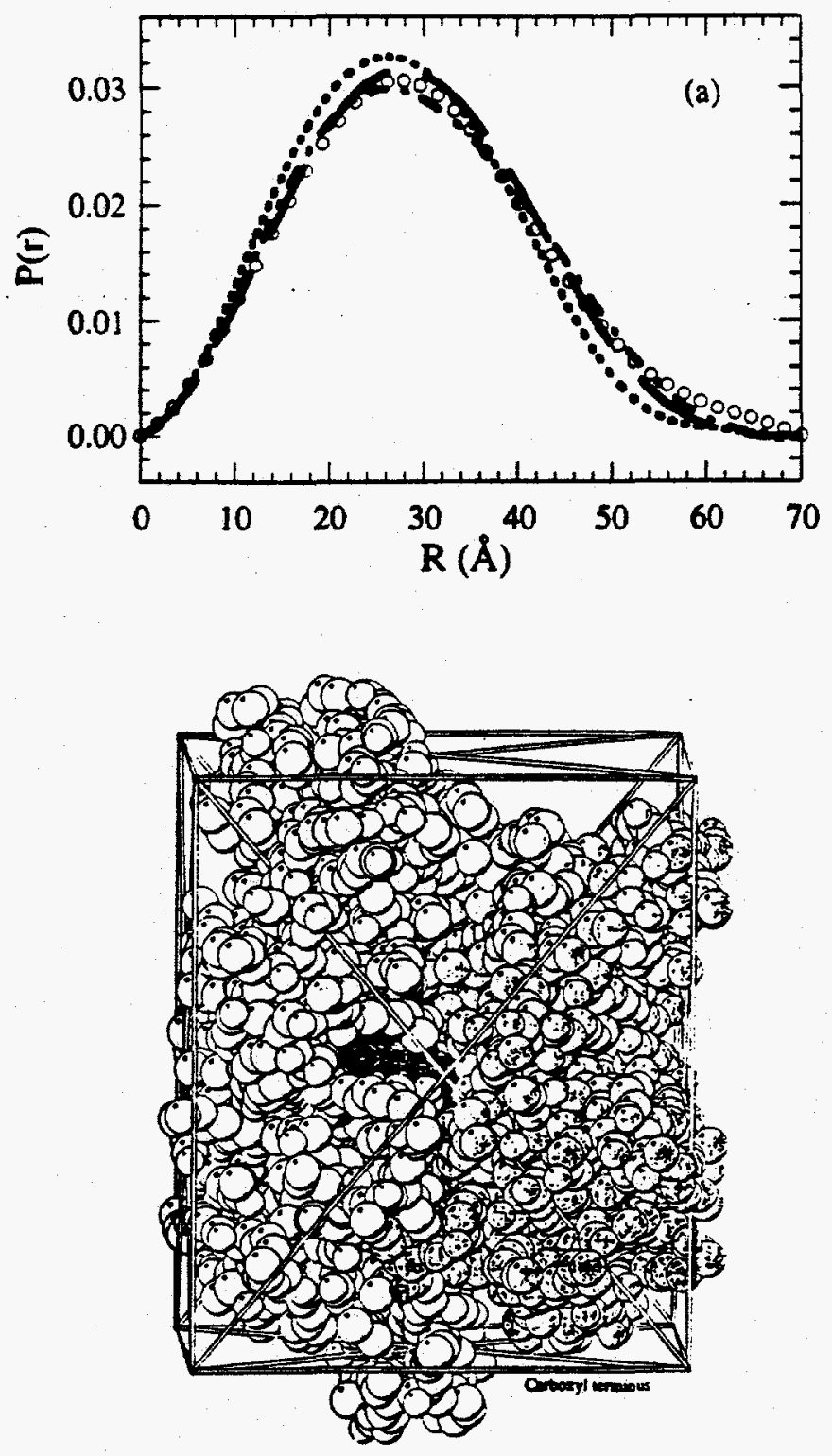

(b)

Figure 3.11. (a) Distance distribution functions for ATPase domain; (o), experimentally derived from $4 \mathrm{mg} / \mathrm{ml}$ protein in the presence of MgADP; (--), computed from crystal coordinates; (- -), computed from the crystal coordinates with a hydration layer added; (- -), calculated from the box model. The experimentally derived $P(r)$ functions in the presence of MgATP is practically superimposable with the experimental curve shown. (b) Superposition of the $56 \times 47 \times 26 \AA$ box model of ATPase on a space filling model of the ATPase domain, drawn with Molscript (Kraulis, 1991). Residues 4-182 and 363-381 are shown in light gray, residues $183-362$ in white and the bound reaction products in dark gray. 
of $R_{g}$. The volume was set equal to the $1.4 \times$ (protein molecular weight) $\times 1.21 \AA^{3} /$ dalton (average protein specific volume). The use of a volume $40 \%$ larger than that computed from the molecular weight results from the increase in volume upon addition of the $3 \AA$ hydration shell necessary to bring the $R_{g}$ and $P(r)$ functions calculated from "dry" atomic coordinates of the ATPase domain into agreement with SAXS data. Using these two constraints $\left(R_{g}\right.$ and volume); dimensions for a series of boxes were generated by allowing $c$ to vary from 52 to $58 \AA$, varying the ratio of a:b from 1.0 to 2.5 in steps of 0.25 . Scattering curves were generated, according to equation [5], for those rectangular prisms with the correct $R_{g}$ and approximate $d_{\max }$ of the experimental value, and the $P(r)$ functions were calculated with the GNOM program (Semenyuk \& Svergun, 1991). The resulting $\mathrm{P}(\mathrm{r})$ functions were compared visually to the experimentally derived function in order to select an optimal set of dimensions. Typically, variations of $-10 \%$ in box dimensions, subject to the constraints described above, showed little apparent difference in agreement between calculated and experimental $P(r)$ functions; larger variations showed clearly discernible differences.

The best dimensions found to model the ATPase domain in the presence of nucleotide were $56 \times 47 \times 26 \AA^{3}$. Superposition of such a box on the crystallographic structure encloses more than $90 \%$ of the protein atoms (Fig. 3.11.b). The $P(r)$ function computed for these dimensions shows reasonable agreement with the experimental curve and with that computed from hydrated atomic coordinates (Fig. 3.11.a).

The same method was used to determine dimensions of a box to represent the peptide binding domain. The best values found for the peptide binding domain were $50 \times 45 \times 13 \AA^{3}$ (Fig. 3.12). These values support the qualitative judgment drawn from the shape of the $\mathrm{P}(\mathrm{r})$ function that the peptide binding domain is oblate rather than prolate.

\subsubsection{Modeling the $60 \mathrm{kDa}$ Fragment}

Two different schemes were used to model the $60 \mathrm{kDa}$ fragment. First, it was modeled as a monomeric protein in both the ATP-bound and ADP-bound forms by combining the ATPase and peptide binding domain models. This reduced the problem to determining (1) the separation between the two domains and (2) their relative orientations. The parallel axis theorem, equation [10], was used to derive an expression that determines the separation of the centers of mass of the ATPase and peptide binding domains, given the $\mathrm{R}_{\mathrm{g}}$ values of both domains and the $60 \mathrm{kDa}$ fragment. This analysis 


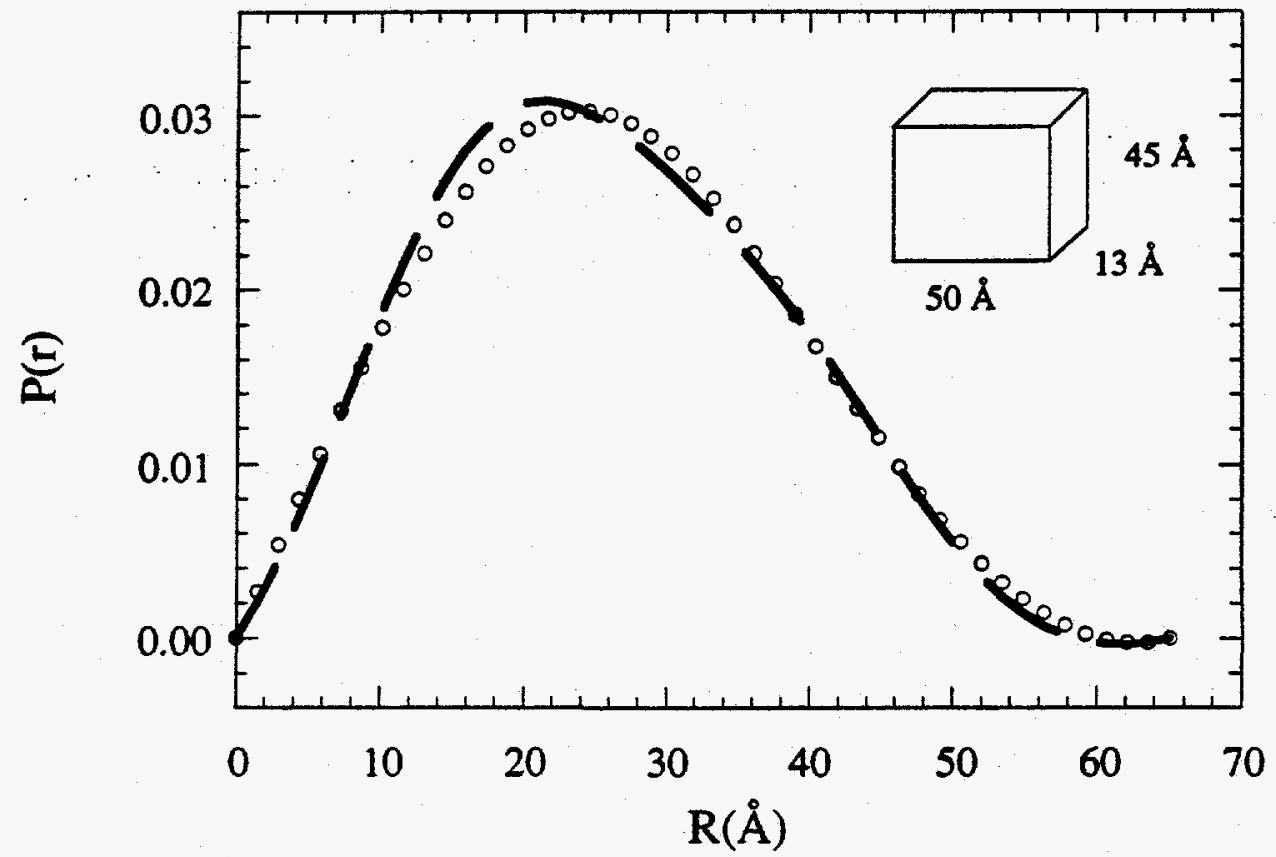

Figure 3.12. Distance distribution functions for the peptide binding domain; (o), experimentally derived from $4 \mathrm{mg} / \mathrm{ml}$ protein; $(-\infty)$, calculated from the $50 \times 45 \times 13 \AA$ box model shown in insert. 
yields a separation of $58 \AA$ for the ADP-bound form and $44 \AA$ for the ATP-bound form of the $60 \mathrm{kDa}$ fragment.

To assess which relative orientations of the two domains were consistent with our data, a selection of orthogonal relative orientations was used with separations (d) of 42 , $44,46 \AA$ and $56,58,60 \AA$ to compute the scattering by equation [8], then calculate $P(r)$ functions. Contact or interpenetration between the two domains is not required in this analysis. Four orientations were examined, "T-shaped", "L-shaped", "face-to-face", and "elongated". Because of the unequivalent dimensions of the prisms, there were several domain placements for each orientation. Representative $P(r)$ function of each orientation is shown in Fig. 3.13. The $P(r)$ function for the ADP-bound form was particularly sensitive to alternative orientations of the two domains. In the case of the ATP-bound form, with a short separation of $-44 \AA$ the two domains readily penetrated each other; the $\mathrm{P}(\mathrm{r})$ functions appeared indistinguishable among different orientations if the two prisms overlapped. Thus, models were justified mainly based on the comparisons with the $\mathrm{P}(\mathrm{r})$ function of the ADP-bound form, and were confirmed from the results of the ATP-bound state. Reasonable agreement was obtained with the "elongated" models where the short axes were parallel to each other and were perpendicular to the line through the centers of mass of the two domains (side-to-side, end-to-end or end-to-side models). In contrast, any models having the shortest axis of one domain parallel to the line through the centers of mass of the two domains ("T-shaped", "L-shaped" and face-toface models) did not agree well. The ATP-induced conformational change was then modeled as a contraction between the domains.

In the second scheme, scattering data for the ADP-bound form of the $60 \mathrm{kDa}$ fragment was modeled as resulting from aggregation of a molecule that retained the overall structure and dimensions of the ATP-bound form. Hypothetical scattering curves for a mixed population of monomers and dimers were computed, with the dimers formed by placing the monomers face-to-face, side-by-side, or end-to-end. It was found that when approximately $30 \%$ of the monomers formed end-to-end dimers, the $P(r)$ function calculated from the hypothetical curve also showed good agreement with the experimentally derived $P(r)$ function (Fig. 3.14). The $R_{g}$ value calculated from the resultant model $P(r)$ function was $34.7 \AA$, in good agreement with the experimental value of $34.3 \AA$. However, the $R_{\mathrm{g}}$ value calculated from the Guinier analysis of the model scattering curves was significantly higher, $37.8 \AA$, reflecting a lack of agreement at low 

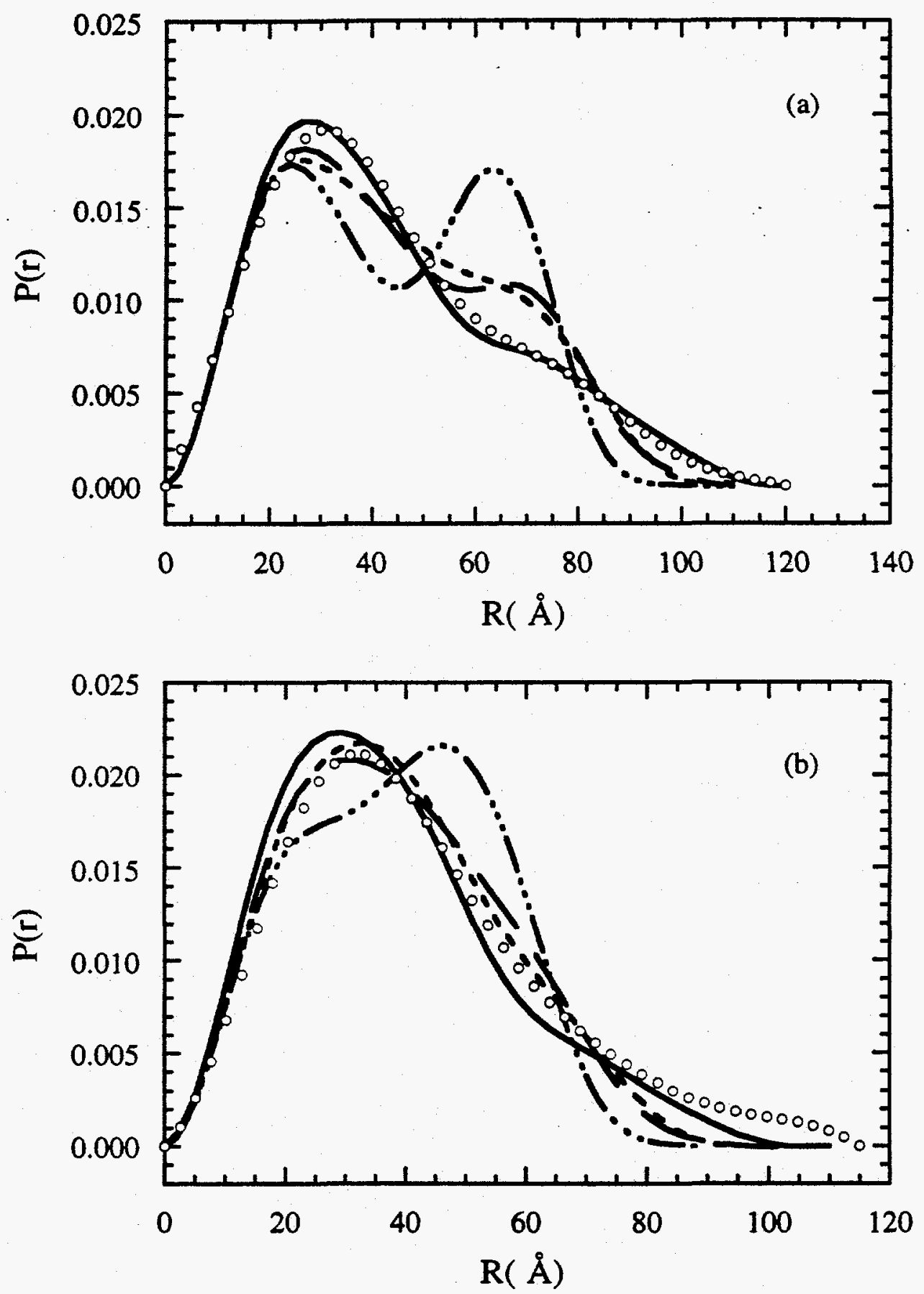

Figure 3.13. Distance distribution functions of the monomeric box models for the 60 $\mathrm{kDa}$ fragment (a) in the presence of MgADP with a separation of $58 \AA$; (b) in the presence of MgATP with a separation of $44 \AA$. (-), "elongated" model; (- -), "Lshaped" model; (- - -), "T-shaped" model; (_ - . -), "paralelled" model. The experimentally derived $P(r)$ functoins are shown in $(O)$ for comparison. 

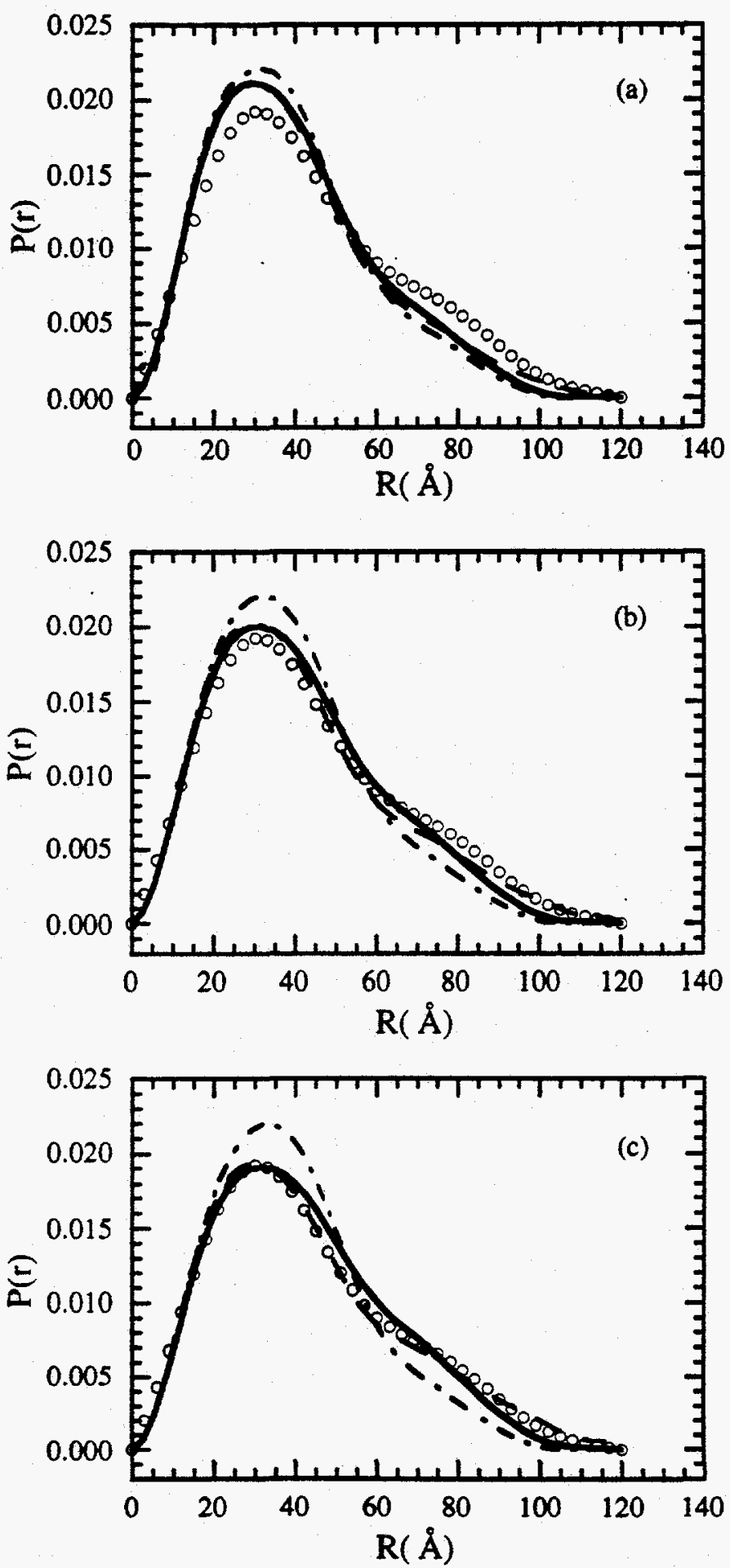

Figure 3.14. Distance distribution functions of the "partial dimer" box models for the 60 $\mathrm{kDa}$ fragment in the presence of MgADP with a separation of $58 \AA$. (a) $10 \%$ of the monomers form dimers; (b) $20 \%$ of the monomers form dimers; (c) $30 \%$ of the monomers form dimers. Dimers were formed -, end-to-end; - -, side-by-side; - - face-to-face. The experimentally derived $P(r)$ functoins are shown in $(O)$ for comparison. 
angle between experimental scattering curves and those computed for a mixture of monomers and dimers.

In summary, agreement between experimental and model $P(r)$ functions for the ADP-bound form of the $60 \mathrm{kDa}$ fragment can be achieved in two ways: either by inducing a large conformational change relative to the ATP-bound form and assuming that the solution is completely monomeric, or by introducing a significant fraction of dimerization in the solution. Arguments that may discriminate between these two alternatives are presented in the discussion.

\subsubsection{Modeling the Full-length hsc70}

To proceed the modeling to the full-length hsc70, the dimensions of the prism of the peptide binding domain were extended to account for the addition of the $\sim 10 \mathrm{kD}$ fragments. For convenience, only three situations were considered where an extension was made along one dimension in each case. Thus, prisms with $45 \times 13 \times 78 \AA^{3}$, or $45 \times 22 \times 50 \AA^{3}$, or $70 \times 13 \times 50 \AA^{3}$ were chosen to represent the carboxyl domain (residues 388-650) including the peptide binding and the $\sim 10 \mathrm{kD}$ fragments. As shown in Fig. 3.15 , the best agreement on experimental and model $P(r)$ functions of both the ATP- and ADP-forms was with a box of $70 \times 13 \times 50 \AA^{3}$; this was achieved by increasing the intermediate dimension of the prism of the peptide binding domain by $25 \AA$.

\subsection{Discussion}

The results presented support the conclusions: (1) the ATPase and peptide binding fragments are relatively oblate; they are linked in a manner that forms a markedly elongated and prolate $60 \mathrm{kDa}$ fragment and full length hsc70; (2) $\mathrm{MgATP}$ induces a substantial decrease in $R_{\mathrm{g}}$ in both hsc70 and the $60 \mathrm{kDa}$ fragment, relative to their MgADP-bound forms; (3) the transition to the ATP-induced conformation occurs more rapidly than ATP hydrolysis, and presumably results from some step of ATP binding, while the rate of the reverse transition to the more elongated state correlates with product release.

Scattering curves measured under steady-state conditions for the $60 \mathrm{kDa}$ fragment and its constituent domains provide the information necessary to construct "low 

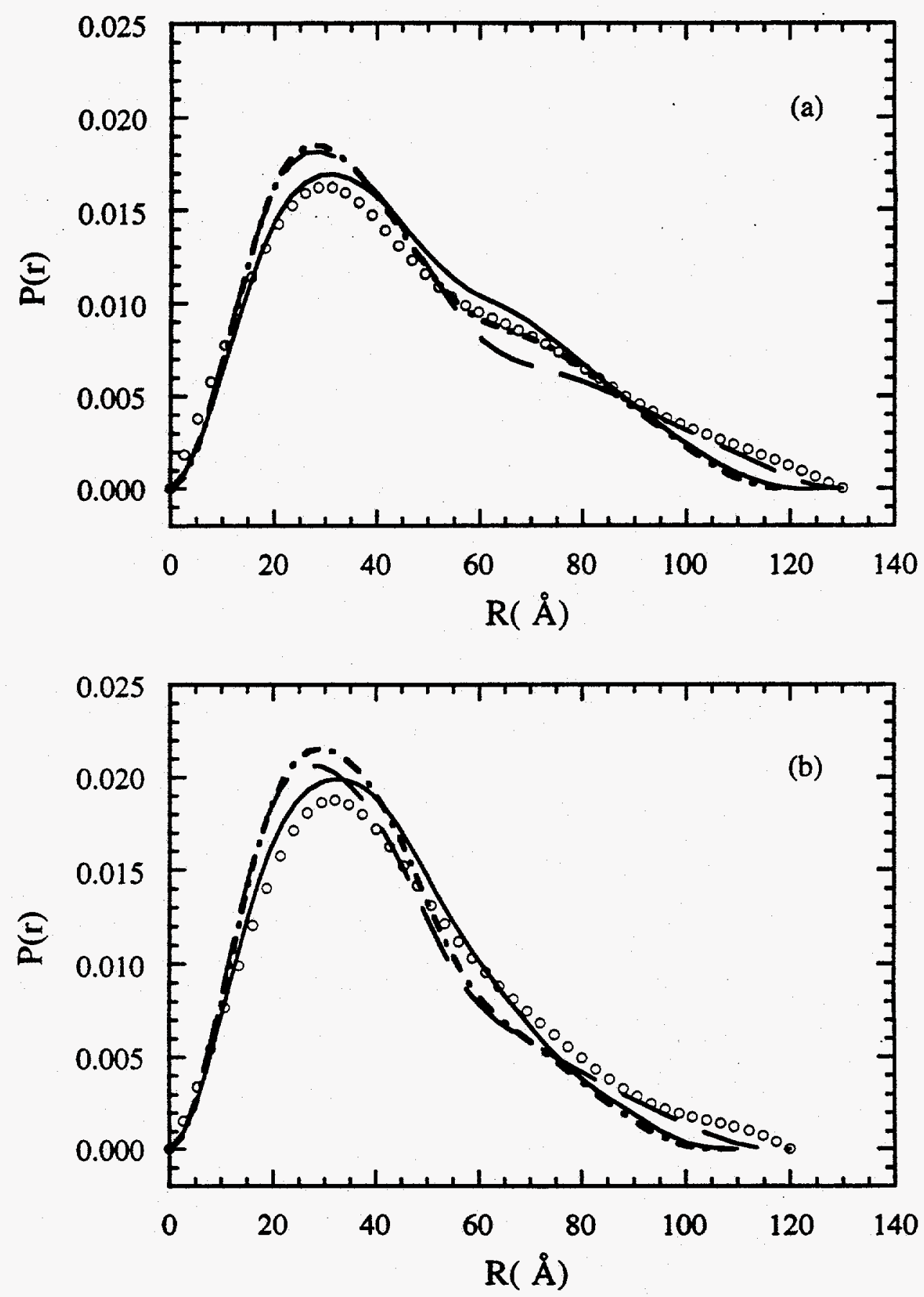

Figure 3.15. Distance distribution functions calculated for the box models for the fulllength hsc70 in the presence of (a) MgADP; (b) MgATP. To make the box model for the carboxyl terminal fragment, the prism representing the peptide binding domain was enlarged along the direction of -, the longest dimension; - - the intermediate dimension; - - the shortest dimension. The experimentally derived $P(r)$ functions are shown in $(O)$ for comparison: 
resolution" models for these fragments. Reliability of the modeling methodology is evidenced by the agreement of the "box" model for the ATPase fragment with the overall dimensions of the crystallographic structure, and the agreement of both the box model and the crystallographic structure with the experimental scattering curves, as long as a -3 $\AA$ layer of hydration is added to the crystallographic coordinates. These schematic models, taken in conjunction with the crystallographic structure of the ATPase fragment, show that a relatively flat peptide binding fragment probably interacts either end-to-end or side-to-side with the ATPase fragment in the ATP-induced form of the molecule. These results exclude models in which the peptide binding domain folds directly onto, and interacts extensively with, the largest faces of the ATPase domain.

Fig. 3.16 shows schemes of the two alternative hypotheses used to model the large increase in $R_{g}$ that occurs going from the ATP-bound forms to the ADP-bound forms of the $60 \mathrm{kDa}$ fragment of hsc70: (1) a large conformational change within monomeric molecules, versus (2) partial dimerization (e.g. $-30 \%$ of the protomers) of the molecules. It should be emphasized in this regard that the scattering curves do not display an upswing at low angle that would be indicative of nonspecific, heterogeneous aggregation (Fig. 3.5).

The partial dimerization model is appealing since hsc70 can be isolated in a dimeric form which can be dissociated with ATP (Freiden, et al., 1992; Schlossman, et al., 1984). However, there are several arguments against the dimerization model for the large $\Delta R_{g}$ we observe. First, partial dimerization would increase the average molecular weight for the ensemble of molecules, and this increase would be detectable as an increase in $\mathrm{I}(0) / \mathrm{c}$ (intensity at zero scattering angle over concentration). For example, if $30 \%$ of the molecules dimerize, an increase of $-30 \%$ in $\mathrm{I}(0) / \mathrm{c}$ is expected (see Section 3.3.1). However, although the sample-to-sample variation of the experimental values for this parameter is relatively large $(-5-10 \%)$ over extended periods of data collection, due to variations in beam intensity and detector response, we do not observe systematically larger values in the presence of ADP versus ATP on samples that are otherwise identical and on which measurements are made close in time (Fig. 3.6).

Secondly, if the larger $R_{\mathbf{g}}$ in the presence of ADP is the result of dimerization, this should be reflected in the dependence of $R_{g}$ on protein concentration; $R_{g}$ should increase significantly with increasing protein concentration and should extrapolate to approximately the same value at zero concentration as seen with ATP-bound protein. 


\section{ATP-Bound Form}
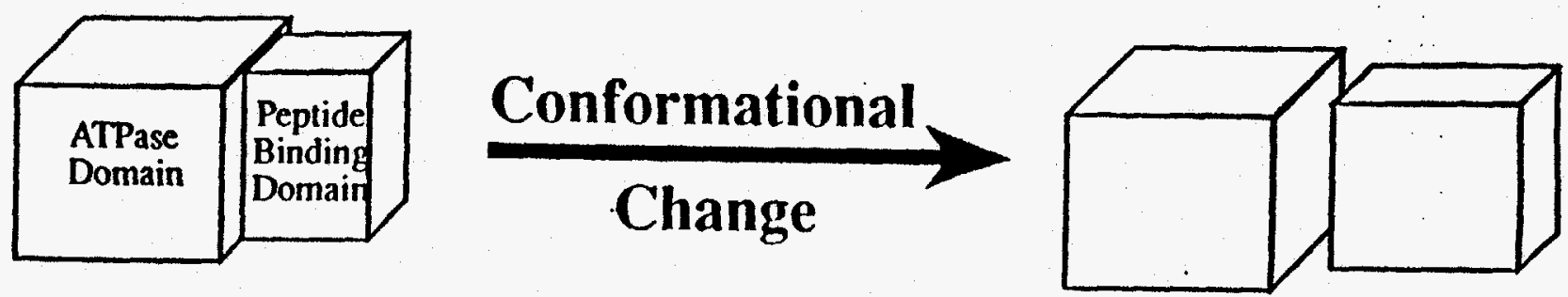

\section{Partial}

\section{Dimerization}
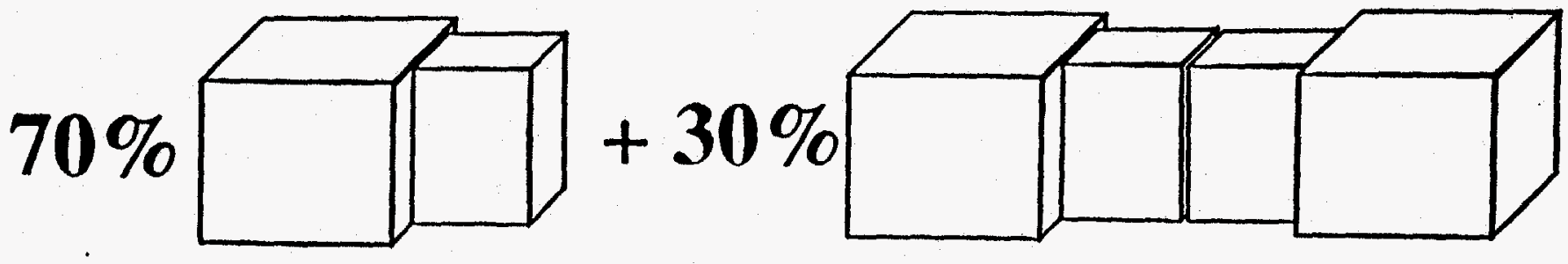

Figure 3. 16 Two alternative mechanisms for the MgATP-induced conformational change in the $60 \mathrm{kD}$ fragment. In the monomeric model, the domain separation decreases from $58 \AA$ to $44 \AA ;$ while in the "partial dimerization" model, $30 \%$ of the protomers form dimers. 
However, the steady state $\mathbf{R}_{\mathbf{g}}$ versus concentration has a similar, approximately level trend in the presence of both ADP and ATP for both hsc70 and th: $60 \mathrm{kDa}$ fragment (Fig. 3.7). Also, calculations show that the $\mathrm{R}_{\mathrm{g}}$ computed in the Guinier approximation would be larger than that computed from the $\mathrm{P}(\mathrm{r})$ function; this is not the case (Table 3.1).

Thirdly, two different biochemical tests support our ability to isolate stable monomers of the $60 \mathrm{kDa}$ fragment. Native gels of samples of the $60 \mathrm{kDa}$ fragment in its ADP-bound form show only minor amounts $(<1 \%)$ of dimer. hsc 70 is more problematic; gels of hsc70 show significant aggregation after storage for extended periods of time. Dynamic light scattering from samples of monomeric hsc70 did not show polydispersity and showed a substantially smaller hydrodynamic radius compared to a sample purified as dimer.

Fourthly, when scattering curves were collected repetitively on samples of the 60 $\mathrm{kDa}$ fragment over many hours, the computed values of $\mathrm{R}_{\mathrm{g}}$ remained stable. In contrast, experiments with hsc 70 showed an increase in $R_{g}$ by 20 hours, consistent with results of gel filtration showing that significant aggregation occurs with full-length protein after storage in solution for this amount of time.

Hence, although we can model the $\mathrm{P}(\mathrm{r})$ function of the ADP-bound $60 \mathrm{kDa}$ fragment with a sample that is a mixture of monomers and dimers of the protein in its ATP-bound conformation, we do not see the effects we would expect from dimerization on values of $\mathrm{I}(0) / \mathrm{c}$, or on the concentration and time dependence of $\mathrm{R}_{\mathrm{g}}$. Nor do we see biochemical evidence for substantial aggregation of the $60 \mathrm{kDa}$ fragment. It is notable in this regard that, taken by itself, the evidence on hsc70 is less compelling. While $I(0) / \mathrm{c}$ shows no dimerization of hsc70 in the presence of ADP, we see substantial variation in measured values of $R_{g}$ as well as biochemical evidence for significant aggregation over time. We have tried to minimize the effect of this on our experiments by minimizing the amount of time between gel filtration of hsc70 samples and scattering measurements on them. The arguments discussed above, which rely more heavily on data from the $60 \mathrm{kDa}$ fragment than on data from hsc70, favor a large, ATP-induced conformational change over partial dimerization as an explanation for the large $\Delta \mathbf{R}_{\mathbf{g}}$ that is observed in both proteins.

Regardless of which particular model ultimately proves to be correct, we discern two significantly different conformational states for both hsc70 and the $60 \mathrm{kDa}$ fragment 
by solution scattering. The state with the larger $R_{g}$ is observed under several sets of conditions, including (i) absence of nucleotide; (ii) presence of saturating levels of MgADP, (iii) presence of slowly hydrolyzable ATP analogs, AMPPNP or AMPPCP, and (iv) with MgATP in the presence of $100 \mathrm{mM} \mathrm{Na}^{+}$rather than $\mathrm{K}^{+}$as a monovalent counter-ion. The other state, the ATP-induced state, is observed only in the presence of MgATP with $\mathrm{K}^{+}$. This correlates with observations from other labs that MgATP induces a conformational change in hsp70 proteins, but slowly hydrolyzable ATP analogs fail to do so, and presence of $\mathrm{K}^{+}$is also required to achieve this conformational change (Banecki, et al., 1992; Liberek, et al., 1991; Palleros, et al., 1992; Palleros, et al., 1993).

Although these data demonstrate two different conformational states for hsc70 and the $60 \mathrm{kDa}$ fragment, it should be noted that the values of steady-state $R_{\mathrm{g}}$ measured in the presence of saturating levels of MgATP represent a mixture of states. Under these conditions, all enzymatic intermediates of the ATPase cycle will be present, and the fraction of each intermediate in solution will be equal to the fraction of time that each molecule spends in that state during the enzymatic cycle. For the $60 \mathrm{kDa}$ fragment, the kinetic constants for the ATPase cycle demonstrate that the majority of the molecules will have MgATP bound under the steady state conditions used in the scattering experiments. ATP binding is rapid $\left(\tau_{1 / 2}<1 \mathrm{sec}\right.$.), hydrolysis is the rate-limiting step of the cycle $\left(\tau_{1 / 2}\right.$ $=400 \mathrm{sec}$.), and following hydrolysis, products are released rapidly $\left(\tau_{1 / 2}<100 \mathrm{sec}\right.$.), allowing ATP to re-bind. Thus greater than $80 \%$ of the $60 \mathrm{kDa}$ fragment molecules will have ATP bound. By implication, since the structural transition to the ATP-induced conformation is apparently brought about by ATP binding, while the reverse transition is predicated on ATP hydrolysis or product release, greater than $80 \%$ of the molecules will be in the more compact, ATP-induced conformation in steady state experiments. This correlates with the observation that, when the transition is synchronized by mixing stoichiometric amounts of MgATP and $60 \mathrm{kDa}$ protein, when essentially all of the protein should be in the ATP-induced conformation, the $R_{g}$ at $t=0,(27.7 \pm 0.5 \AA$, estimated from a double exponential fit to $R_{g}$ versus time), is equal to that observed with MgATP in steady-state experiments $(27.9 \pm 0.5 \AA)$.

For hsc70, the ATP hydrolysis and $P_{i}$ release steps require approximately equal time ( $\tau_{1 / 2}=240$ and $180 \mathrm{sec}$. respectively), while ADP release is relatively rapid ( $\tau_{1 / 2}=$ $24 \mathrm{sec}$.). In the presence of excess MgATP, hsc70 will be an approximately 50:50 mixture of protein with ATP bound and protein with ADP $+P_{i}$ bound. Depending on whether ATP hydrolysis, or alternatively, $P_{i}$ or ADP release facilitates the transition from 
the compact to the more elongated state, hsc70 would be either a $-50: 50$ mixture of protein in two different conformations, or alternatively, $>90 \%$ in the ATP-induced form. The kinetics of the change of $R_{\mathrm{g}}$ after mixing stoichiometric amounts of hsc70 and MgATP show that the $R_{g}$ observed at early times is equal within error to that observed with MgATP under steady state conditions, demonstrating that the transition to the elongated form is predicated on product release rather than ATP hydrolysis. The rate constant inferred from our data is consistent with this interpretation.

The conformational switch which induces the transitions between the two states presumably relies on the ability of nucleotide, which binds at the base of a cleft in the ATPase domain, to transmit a conformational shift to the periphery of this domain. However, no major change in $\mathrm{R}_{\mathrm{g}}$ is observed between ATP-bound and ADP-bound 44 $\mathrm{kDa}$ ATPase fragment. This argues that any ATP-induced conformational change in this fragment is relatively subtle. An increase in $R_{g}$ is observed in the absence of nucleotides, suggesting that the cleft of the ATPase fragment opens when nucleotide is released. However, this does not appear to correlate with the transition to or from the ATP-induced state.

When modeled as a large conformational change in a monomeric $60 \mathrm{kDa}$ fragment, the change from the ATP-induced state to the more elongated ADP-bound state involves an increase of $\sim 14 \AA$ in the center-to-center distance between the two domains. This suggests that the ATPase and peptide binding domains disassociate and may have few if any noncovalent interactions in the more extended state. These conclusions can be extended to hsc70, whose behavior is similar to that of the $60 \mathrm{kDa}$ fragment. The solution scattering data demonstrate that, at the level of substantial structural changes in the protein, the $60 \mathrm{kDa}$ fragment mimics hsc70. A caveat to be emphasized at this point is that the carboxyl-terminal $\sim 100$ amino acid residues of hsc70 that are not present in the $60 \mathrm{kDa}$ fragment may be involved in significant interactions to which the solution scattering experiments, being relatively coarse in resolution, are not sensitive.

The low peptide affinity state of DnaK characterized by Palleros et al. shares two significant features with the ATP induced state described here: (1) both are induced by ATP before hydrolysis occurs and (2) both appear to persist for a matter of minutes (Palleros, et al., 1993). Although it is appealing and reasonable to suggest that the two different states observed in hsc70 and the $60 \mathrm{kDa}$ fragment correspond to the high- 
peptide-affinity and low-peptide-affinity states of the proteins, this has not been demonstrated directly.

A question remaining to be addressed is which chemical steps of the ATPase cycle transduce the conformational changes? The transition to the ATP-induced structure both in the $60 \mathrm{kDa}$ fragment and in hsc70 occurs more rapidly than ATP hydrolysis and appears to be correlated with ATP binding. Crystallographic work on the wild-type ATPase fragment shows how both AMPPNP and ADP $+P_{i}$ bind to this fragment. These structures, along with structures of several mutants with either ATP or ADP + $P_{i}$ bound, show both a "pre-hydrolysis" state and the "post-hydrolysis" state for the nucleotide. There is no significant difference in the overall tertiary structure of the ATPase fragment between these two states. To the extent the crystallographic structure of the isolated ATPase fragment represents its structure in conjunction with the peptide binding domain in hsc70 and the $60 \mathrm{kDa}$ fragment, this suggests that it must have an additional (that is, not yet crystallized) conformational state that is accessed by ATP but not by AMPPNP. It appears that AMPPNP is unable to stabilize the ATP-induced state in either crystal or solution studies. In the hydrolysis pathway proposed for ATP, the nucleotide first binds in the same manner as AMPPNP, and then rearranges to allow the phosphates to form a $\beta, \gamma$-bidentate complex with the $\mathrm{Mg}^{2+}$ ion in the active site. Hydrolysis then proceeds by an in-line attack mechanism. By inference, the postulated $\beta, \gamma$-bidentate complex of ATP is a candidate for a specific ATP conformation which provokes a structural shift which is then transduced from the nucleotide to the periphery of the ATPase fragment.

As for the step which precedes the converse structural transition, from the ATPinduced state to the more elongated state, our current data limit the possibilities to product release, but cannot discriminate between $\mathrm{P}_{\mathrm{i}}$ or ADP release for this role; both are equally viable candidates for the biochemical step that triggers a conformational change. In the context of the structural similarity of the hsc70 ATPase fragment with actin, it is intriguing to see whether $P_{\mathrm{i}}$ release induces a subtle shift in the ATPase domain of hsc70 that destabilizes the interactions with the peptide binding domain, in the same manner that $P_{i}$ release apparently induces a subtle structural change in actin that destabilizes the protein-protein interactions in the actin filament (Korn, et al., 1987). 


\subsection{Acknowledgments}

This work was supported by National Institutes of Health Grants RR-01209 (to K. O. H.) and GM-39928 (to D. B. M.). SSRL operations are funded by the Department of Energy, Office of Basic Energy Sciences. The Biotechnology Program is supported by the National Institutes of Health, Biomedical Research Technology Program, National Center for Research Resources. Additional support is provided by the Department of Energy, Office of Health and Environmental Research. I wish to thank Dr. Sigurd Wilbanks for providing hsc70 and its subfragments samples, and members of the McKay's group for additional sample preparation and for their assistance with data collection. I would like to thank Prof. McKay, who made things smooth and going including every experiment detail - sample availability, data collection and analysis, for his advice and encouragement. I appreciate Dr. Hirotsugu Tsuruta for his support during the scattering experiments and his taking care of the beamline equipment. Acknowledgment is extended to Dr. Dimitri Svergun at EMBL for the GNOM and CRYSOL programs, and to Daniel Segel for his assistance in scattering setup. I would like to thank Dr. David Eliezer, without him this work might not have started.

\subsection{References}

Anderegg, J. W., Beeman, W. W., Shulman, S., \& Kaesberg, P. (1955). J. Am. Chem. Soc., 77, 2927-2937.

Banecki, B., Zylicz, M., Bertoli, E., \& Tanfani, F. (1992). J. Biol. Chem., 267, 25051 25058.

Beckman, R. P., Mizzen, L. A., \& Welch, W. J. (1990). Science, 248, 850-854.

Bennett, W. S. J., \& Steitz, T. A. (1980). J. Mol. Biol., 140, 211-230.

Blond-Elguindi, S., Cwirla, S. E., Dower, W. J., Lipshutz, R. J., Sprang, S. R., Sambrook, J. F., \& Gething, M. J. (1993). Cell, 75, 717-728.

Chappell, T. G., Konforti, B. B., Schmid, S. L., \& Rothman, J. E. (1987). J. Biol. Chem., 262, 746-751.

Chappell, T. G., Welch, W. J., Schlossman, D. M., Palter, K. B., Schlesinger, M. J., \& Rothman, J. E. (1986). Cell, 45, 3-13.

Chirico, W. J., Waters, M. G., \& Blobel, G. (1988). Nature, 332, 805-810.

Deluca-Flaherty, C., McKay, D. B., Parham, P., \& Hill, B. L. (1990). Cell, 62, 875-887. 
Deshaies, T. J., Koch, B. D., Werner-Washburne, M., Craig, E. A., \& Schekman, R. (1988). Nature, 332, 800-805.

Flaherty, K. M., DeLuca-Flaherty, C. M., \& McKay, D. B. (1990). Nature, 346, 623-628.

Flaherty, K. M., McKay, D. B., Kabsch, W., \& Holmes, K. C. (1991). Proc. Natl. Acad. Sci. USA, 88, 5041-5045.

Flaherty, K. M., Wilbanks, S. M., Deluca-Flanerty, C., \& McKay, D. B. (1995). J. Bio. Chem, , 269(17), 12899-12907.

Fletterick, R. J., Bates, D. J., \& Steitz, T. A. (1975). Proc. Natl. Acad. Sci. USA, 72, 3842.

Flynn, G. C., Chappell, T. G., \& Rothman, J. E. (1989). Science, 245, 385-390.

Flynn, G. C., Pohl, J., Flocco, M. T., \& Rothman, J. E. (1991). Nature, 353, 726-730.

Freiden, P. J., Gaut, J. R., \& Hendershot, L. M. (1992). EMBO J., 11, 63-70.

Fujisawa, T., Ueki, T., \& Iida, S. (1990). J. Biochem., 107, 343-351.

Glatter, O., \& Kratky, O. (Ed.). (1982). Small Angle X-ray Scattering. New York: Academic Press Inc.

Gragerov, A., Zeng, L., Zhao, X., Burkholder, W., \& Gottesman, M. E. (1994). J. Mol. Biol., 235, 848-854.

Ha, J.-H., \& McKay, D. B. (1994). Biochemistry, 33, 14625-14635.

Hiragi, Y., \& Ihara, S. (1981). Acta Cryst., A37, 378-382.

Kim, D., Lee, Y. J., \& Corry, P. M. (1992). J. Cell Physiol., 153, 353-361.

Korn, E. D., Carlier, M.-F., \& Pantaloni, D. (1987). Science, 238, 638-644.

Kraulis, P. (1991). J. Appl. Cryst., 24, 946-950.

Langer, T., Lu, C., Echols, H., Flanagan, J., Hayer, M. K., \& Hartl, F.-U. (1992). Nature, 356, 683-689.

Liberek, K., Skowyra, D., Zylicz, M., Johnson, C., \& Georgopoulos, C. (1991). J. Biol. Chem., 266, 14491-14496.

McKay, D. B. (1993). Adv. Prot. Chem., 44, 67-98.

Morimoto, R. I., Tissieres, A., \& Georgopoulos, C. (Ed.). (1990). Stress Proteins in Biology and Medicine. New York: Cold Spring Harbor Laboratory Press.

Morimoto, R. I., Tissieres, A., \& Georgopoulos, C. (Ed.). (1994). The Biology of Heat Shock Proteins and Molecular Chaperones. New York: Cold Spring Harbor Laboratory Press.

Morshauser, R. C., Wang, H., Flynn, G. C., \& Zuiderweg, E. R. P. (1995). Biochemistry, 34, 6261-6266.

Nover, L. (Ed.). (1984). Heat Shock Response of Eukaryotic Cells. New York: Springer-Verlay. 
O'Brien, M. C., \& McKay, D. B. (1993). J. Biol. Chem., 268, 24323-24329.

Palleros, D. R., Reid, K. L., McCarty, J. S., Walker, G. C., \& Fink, A. L. (1992). J. Biol. Chem., 267, 5279-5285.

Palleros, D. R., Reid, K. L., Shi, L., Welch, W. J., \& Fink, A. L. (1993). Nature, 365, 664-666.

Rice, M., \& Wakatsuki, S. (1991). SSRLSLAC user manual .

Ritossa, F. (1962). Experientia, 18, 571-573.

Scherer, P. E., Krieg, U. C., Hwang, S. T., Vestweber, D., \& Schatz, G. (1990). EMBO J., 9, 4315-4322.

Schlossman, D. M., Schmid, S. L., Braell, W. A., \& Rothman, J. E. (1984). J. Cell Biol., 99, 723-733.

Semenyuk, A. V., \& Svergun, D. I. (1991). J. Appl. Cryst., 24, 537-540.

Svergun, D. I., Barberato, C., \& Koch, M. H. (1995). J. Appl. Crystallgr, submitted for publication.

Tissieres, A., Mitchell, H. K., \& Tracy, U. M. (1974). J. Mol. Biol., 84, 389-398.

Wakatsuki, S., Hodgson, K. O., Eliezer, D., Rice, M., Hubbard, S., Gillis, N., \& Doniach, S. (1992). Rev. Sci. Instrum., 63, 1736-1740.

Wang, T., Chang, J., \& Wang, C. (1993). J. Biol. Chem., 268(35), 26049-26051.

Wilbanks, S. M., DeLuca-Flaherty, C., \& McKay, D. B. (1994). J. Biol. Chem., 269, 12893-12898. 
Chapter 4

Equilibrium Studies of Lysozyme Unfolding by Solution X-Ray Scattering: Evidence for A Stable Folding Intermediate 


\subsection{Introduction and Background}

The protein folding problem has been a major challenge in biophysical research. More specifically, the protein folding problem refers to the questions why and how a protein of given amino acid sequence folds into a specific three-dimensional native conformation. What determines which of the large number of possible conformations is adopted by a protein as its unique native conformation? How does the polypeptide chain search into the native conformation given the astronomical number of possible conformations that a given amino acid sequence composed of 20 amino acids can assume? What are the rules governing the relationship between amino acid sequence and threedimensional structure? The ultimate goals in protein folding research are to predict threedimensional structures from the primary sequences, to design proteins to achieve desired structures and therefore to perform specific functions.

The study of the protein folding problem began with Anfinsen's pioneering work in the late 1950's. In 1973, based on the observations that an unfolded polypeptide, ribonuclease A, could spontaneously refold to form a native protein with full biological activity in vitro, Anfinsen proposed that the necessary information required to define the three-dimensional structure of a protein is contained in the primary sequence of the polypeptide (Anfinsen, 1973). However, unlike the straightforward genetic DNA codes for protein synthesis, the folding codes for proteins appears formidably complex.

The theoretical and computational approaches to the folding problem can be roughly classified into two major categories. One is molecular dynamics simulation which is based on classsical statistics and quantum mechanics calculation (see Dill, et al., 1995 for a recent review). Another approach is data-based predictive algorithms based on sequence homology in the protein data base (See Blundell \& Johnson, 1993 for a recent review).

Experimentally, at present a more tractable problem is to study the folding mechanisms, i.e. the processes or pathways by which proteins adopt their native conformations. Much of the work in this field has been devoted to this kind of studies. Like any chemical reactions, understanding of a folding mechanism includes the elucidation of the pathway(s) between the native folded state and the unfolded state, the intermediates and the transition states along the folding pathway(s). Currently, there are two major generalized models for the folding mechanisms. The framework model suggests that an early intermediate with the framework of secondary structure is formed before a structure 
with the native-like tertiary fold and stable secondary structure is formed (Ptisyn, 1973). The modular assembly model postulates that microdomains of a protein fold transiently and independently, and they diffuse, collide and coalesce into the stable native form (Karplus \& Weaver, 1976; Kim \& Baldwin, 1990).

Kinetics intermediates on the folding pathway have been identified mainly by kinetic CD, fluorescence and pulsed hydrogen labeling techniques (see Schmid, 1993 for a recent review). Early intermediates are formed within milliseconds after the initiation of refolding, and are normally beyond the time resolution of most techniques. Late intermediates, formed at a longer time scale, hundreds of milliseconds or even longer after refolding, reveal some structural information. However, structural characterizations of kinetic intermediates are intrinsically difficult due to their transient occurrence. An alternative is to investigate stable intermediates under different stages of equilibrium folding.

The first evidence for intermediate conformation between the rigid native structure and the random coil unfolded state was reported in 1967. It was found that temperature or acid denaturation of a protein transformed the native state to a state which could be further unfolded by stronger denaturants such as urea or guanidine hydrochloride (Aune, et al., 1967). The important demonstrations for the existence of intermediate folding species under equilibrium conditions came from equilibrium unfolding studies of $\alpha$-lactalbulmin where two distinct transitions were observed by far- and near-UV circular dichroism (Kuwajima, et al., 1976). The intermediate was found to have a native secondary structure but without a rigid tertiary structure.

The term "molten globule" was first proposed to describe some intermediate states at low pH (Ohgushi \& Wada, 1983). The acid form is found to be nearly as compact as the native state with native-like secondary structure content, but without rigid tertiary structure. Since its emergence, the molten globule has attracted the most attention among various folding intermediate states. A range of physical techniques, particularly NMR and $\mathrm{x}$-ray scattering, has been applied to characterize this type of intermediate, revealing different aspects of physical properties of this species. The current model for molten globule intermediates is that the molten globule state not only maintains some of the native-like secondary structure, but also preserves the native-like folding pattern; however, it lacks the tight packing of side chains and has looser structure than the native state (Ptisyn, 1993). 
Study of equilibrium intermediates was initially motivated by the belief that these stable intermediates under different stages of equilibrium folding might mimic the transiently occurring kinetic counterparts in the folding pathway. Compact intermediates identified in the folding pathway are found to share similar structural features, i.e. substantial secondary contents and lack of rigid tertiary structure, to the equilibrium analog. Correlations between the kinetics and equilibrium intermediates have been observed in some of the most frequently studied proteins, such as $\alpha$-lactalbumin, cytochrom $c$, hen lysozyme and interleukin- $\beta$. A kinetic intermediate in the apomyoglobin folding is found to have the same NH-protection pattern as an equilibrium molten globule (Jennings \& Wright, 1993). It becomes established that the molten globule is the common kinetic intermediate on the folding pathway (Kim \& Baldwin, 1990; Ptitsyn, 1995a).

In this chapter, the $x$-ray scattering technique has been applied to study ureainduced lysozyme unfolding under equilibrium conditions. A partially folded intermediate of hen lysozyme has been found which is induced by a combination of reduced $\mathrm{pH}$ and denaturant. The intermediate is relatively compact; however, there is a signature which suggests some random coil component.

Lysozyme. Hen egg-white lysozyme is one of the earliest characterized and most studied globular proteins. Its 129 amino-acid residues fold into two distinctive structural domains as shown in Fig. 4.1: the $\alpha$ domain consists of helices A-D and a $3^{10}$ helix, the $\beta$ domain includes a long triple-stranded $\beta$ sheet, a short double-stranded $\beta$ sheet, a $3^{10}$ helix and a long loop (Blake, et al., 1965; Radford, et al., 1992). The structure of lysozyme is further stablized by the presence of four disulfide bonds: two $(6-127,30-115)$ within the $\alpha$ domain, one (76-94) between the $\alpha$ and $\beta$ domains, and one (64-80) within the $\beta$ domain. Two proline residues at positions 70 and 79 are trans in the native conformation. It is interesting to know that most issues on the folding (folding of $\alpha$ helices and $\beta$ sheets, formation of disulfide bonds, and the effect of proline isomerization on folding) are present in such a small protein.

Kinetics and equilibrium aspects of lysozyme folding have been under extensive investigation (Dobson, et al., 1994; Tanford, 1970). To decipher the folding pathway, it is important to characterize folding intermediates. For hen lysozyme, kinetic intermediates have been identified in the early stage $(<100 \mathrm{~ms}$ ) of the refolding pathway (Kato, et al., 1981; Radford, et al., 1992). The $\alpha$ domain of the intermediates appears to have some 


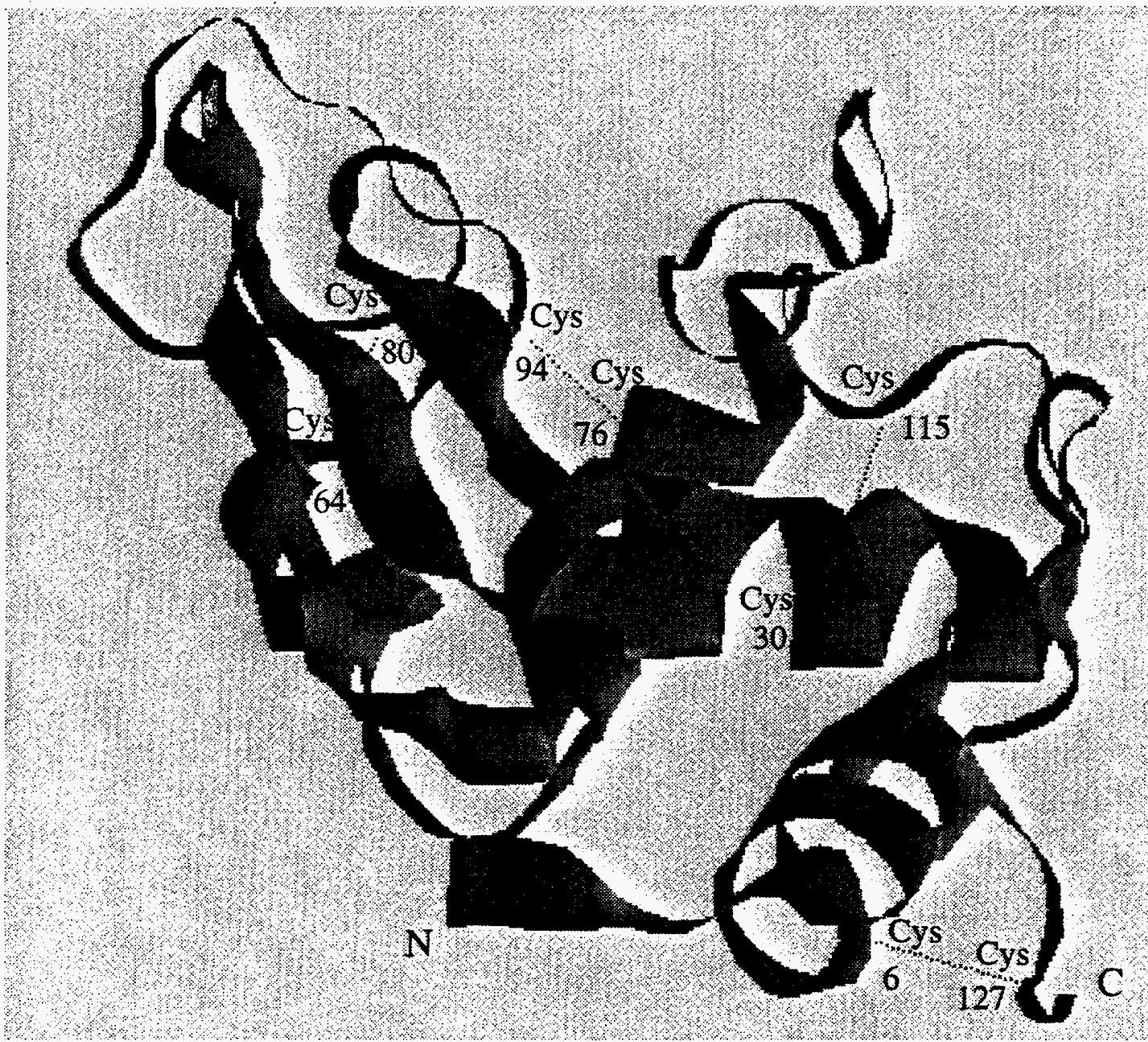

Figure 4.1. Three dimensional structure of hen lysozyme (entry PDB6LYZ.ENT, Brookhaven Protein Data Bank). The four disulfide bridges are shown along with the corresponding Cysteine residues. 
characteristics of a molten globule, i.e., with an extensive secondary structure but a distorted, far from native-like tertiary structure, while the conformation of the $\beta$ domain is still highly labile (Radford, et al., 1992). Structural studies of these intermediates are difficult because of their transient occurrence.

An alternative approach is to study the partially folded states as evidence is growing that they may resemble kinetic intermediates (Dobson, 1994; Ptitsyn, 1995b). However, the equilibrium unfolding transition of lysozyme, mostly induced by thermal or GdmHCl denaturation, has been traditionally approximated by a two-state model due to the high unfolding cooperativity (Aune \& Tanford, 1969). Stable partially folded intermediates were not visibly present under those circumstances. Recently, some stable intermediates have been reported on either lysozyme from other species, such as equine and human (Dael, et al., 1993; Haezebrouck, et al., 1995) as well as a homologous protein $\alpha$ lactalbumin (Baum, et al., 1989), or hen lysozyme using the mild denaturant trifluoroethanol (TFE) at pH 2.0 (Buck, et al., 1993; Dael, et al., 1993). Structures of these partially folded states, determined by NMR, far- and near-UV CD and fluorescence spectroscopies, show molten globule features. They have substantial $\alpha$ helical content and a distorted tertiary fold in the $\alpha$ domain and little structure in the $\beta$ domain, and resemble that of the early kinetic intermediate in hen lysozyme. Additional evidence for intermediates is also documented in the literature: thermal unfolding curves of hen lysozyme are not identical, as observed by absorption at $292 \mathrm{~nm}$ and optical rotation at $546 \mathrm{~nm}$ at pH 2.2 (Wetlaufer, 1962), and by $C D$ measurements at $222 \mathrm{~nm}$ and $270 \mathrm{~nm}$ at $\mathrm{pH} 1.0$ (Haezebrouck, et al., 1995). A population of $25 \%$ of an intermediate was suggested from the latter study. An early NMR study found that in hen lysozyme, regions containing histidine and arginine residues unfolded at a lower urea concentration than portions with methionine at pH 2.8 in urea solution (Bradbury \& King, 1969).

Compactness is one of the important properties pertinent to the structure of protein molecules in solution, and it also characterizes the degree of folding. Small angle $\mathrm{x}$-ray scattering (SAXS) is one of the few techniques that provides a direct measurement of compactness since SAXS gives overall geometric information such as the size and shape of the molecule. Application of SAXS to the study of protein folding has been proven successful (Eliezer, et al., 1993; Kataoka, et al., 1995; Lattman, 1994), and information provided from such studies is complementary to that obtained by other techniques, such as $\mathrm{CD}$, fluorescence and NMR, in the folding study. 


\subsection{Experimental Procedures}

\subsubsection{Sample Preparations}

Hen egg-white lysozyme was purchased from the Sigma Chemical Company, St. Louis, MO. A lysozyme stock solution of $\sim 70 \mathrm{~g} /$ was dialyzed extensively at $4^{\circ} \mathrm{C}$ against a large volume of buffer (100 mM Na-citrate, $100 \mathrm{mM} \mathrm{NaCl}$ at pH 2.9;100 mM Naphosphate, $100 \mathrm{mM} \mathrm{NaCl}$ at $\mathrm{pH} 2.0$; or $100 \mathrm{mM} \mathrm{Na}$-acetate, $100 \mathrm{mM} \mathrm{NaCl}$ at $\mathrm{pH} 3.8$ ). The solution was concentrated back to $\sim 70 \mathrm{~g} / \mathrm{l}$ using an Amicon concentrator (Amicon, Danvers, MA) when necessary. The solution was ultra-centrifuged at $34,000 \mathrm{rpm}$ in Beckman Ti50 rotor for at least $60 \mathrm{~min}$ at $10^{\circ} \mathrm{C}$ to remove any protein precipitate and dust particles. The $\mathrm{pH}$ of a $10 \mathrm{M}$ urea stock solution in the same buffer was adjusted to 2.9 or 2.0 , and was filtered through a $0.2 \mu \mathrm{m}$ filter. The titration samples of different urea concentrations were then prepared volumetrically from the stock solutions. All unfolded protein samples were allowed to equilibrate for at least five hours prior to both scattering and $\mathrm{CD}$ experiments. The final lysozyme concentrations, measured by UV absorption $\left(\varepsilon_{280}=2.64 \mathrm{mg} / \mathrm{ml}\right)$, were about $7 \mathrm{mg} / \mathrm{ml}$ for scattering experiments. All the samples containing urea were used within 24 hours. The lysozyme solutions were used within 48 hours. To prepare reduced unfolded lysozyme, the unfolded sample (in $8 \mathrm{M}$ urea for more than 5 hours) was incubated in $80 \mathrm{mM}$ dithiothrietol solution for up to 3 hours.

Heparin (from Calbiochem, San Diego, CA) was further purified by gel filtration using Sephacryl S-200. The buffer for heparin was $0.5 \mathrm{M} \mathrm{NaCl}, \mathrm{pH} 2.5$ under which conditions heparin is assumed to exist as a random coil by viscosity measurements (Liberti \& Stivala, 1967).

Although $\mathrm{GdmHCl}$ is a stronger chemical denaturant for proteins than urea, it is not ideal for SAXS studies because of the relatively large absorption of $x$-rays by chlorine. As shown in Fig. 4.2, transmittance of $x$-rays decrease dramatically with increased $\mathrm{GdmHCl}$ concentration. The absorption of a $6 \mathrm{M} \mathrm{GdmHCl}$ solution is about 7.5 times higher than the same solution without $\mathrm{GdmHCl}$. On the other hand, the presence of urea has little effect on absorption. Thus, urea is used instead as the denaturant in the equilibrium (this chapter) and kinetic (Chapter 5) studies. 

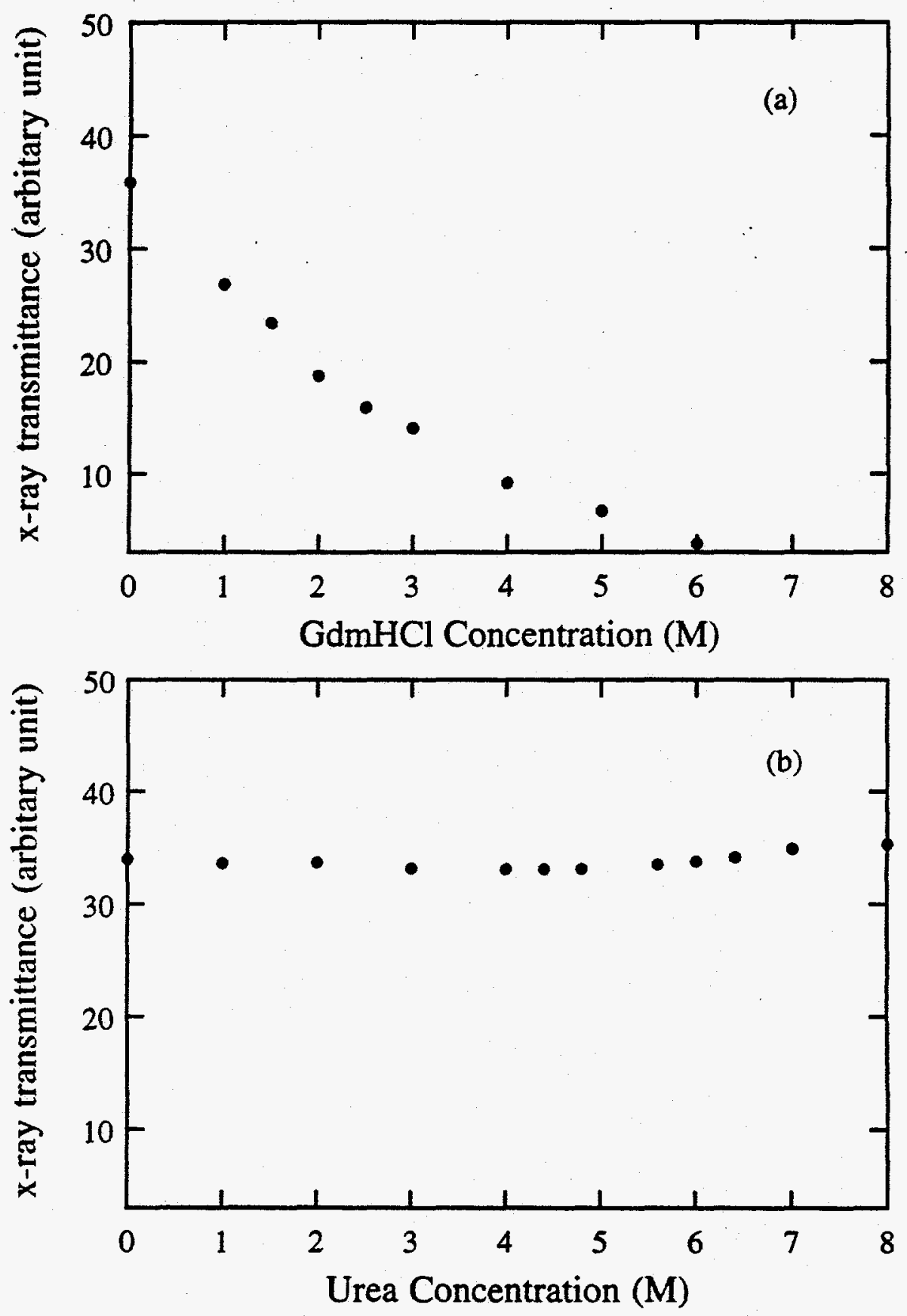

Figure 4.2. Absorption of $\mathrm{x}$-rays by $\mathrm{GdmHCl}$ and urea solution. Urea is prefered for SAXS studies as it does not absorb much of the $x$-rays. 


\subsubsection{Data Collection}

$\mathrm{X}$-ray scattering data were collected at beamline 4-2 at Stanford Synchrotron Radiation Laboratory. The x-ray energy was selected by a pair of Si [111] monochromators at $8980 \mathrm{eV}$, or by a pair of $\mathrm{Mo} / \mathrm{B}_{4} \mathrm{C}$ multilayers (Stephenson, 1988). In the case of multilayers, aluminum foils were used to attenuate beam intensity when necessary. To monitor the fluctuations of the $\mathrm{x}$-ray intensity, a short length ion chamber and a scintillation counter were placed before and after the sample cell, respectively. The linear position sensitive proportional counter for the scattering data was filled with either $70 \% \mathrm{Ar}+30 \% \mathrm{CO}_{2}$ or $80 \% \mathrm{Xe}+20 \% \mathrm{CO}_{2}$. When using the Ar mixture as detector gas and with monochromatic beam, typical counting rates were $\sim 4,400$ counts per second (cps) for buffer and 6,500 cps for the sample; while using Xe mixture as detector gas and with broad-band pass beam, counting rates increased to $-30,000 \mathrm{cps}$ for buffer and $\sim 50,000 \mathrm{cps}$ for the sample. The camera length was calibrated to be $230 \mathrm{~cm}$ for the normal SAXS configuration and $150 \mathrm{~cm}$ for the short camera-length setup, using a cholesterol myristate sample.

At the early stage of these serial experiments, data were collected using static sample cells as described in Section 3.2.2. Depending on the intensity of the incident $x$-ray beam, the exposure time was limited to 8 or 10 minutes since a time dependence of the scattering profile at the small angle region was observed after $\sim 12$ minutes. For samples containing high concentration of denaturants, the difference in counting rate between the sample and the buffer decreased to less than $1,000 \mathrm{cps}$ in some cases, hence a longer collection time was required to improve data statistics. A flow cell was then developed to extend data acquisition time to longer than 30 minutes by circulating protein solution continuously from a reservoir through a $1.3 \mathrm{~mm}$ scattering-path observation cell with 10 $\mu \mathrm{m}$ thick mica windows. The flow rate was adjusted in such a way that protein molecules would be exposed to the $\mathrm{x}$-ray beam for less than one second during each circulation. The temperature of both the static and the flow cells was controlled by circulating ethylene glycol through the cell holder, and was set to be $20^{\circ} \mathrm{C}$ for the cell, and also for the reservoir in the case of the flow cell.

$\mathrm{CD}$ data were measured at $222 \mathrm{~nm}$ at $20^{\circ} \mathrm{C}$ with an Aviv 60DS spectropolarimeter using $1 \mathrm{~mm}$-path cell. Protein concentration was determined to be $\sim 40 \mu \mathrm{M}(-0.6 \mathrm{mg} / \mathrm{ml})$. 


\subsection{Data Analysis}

\subsubsection{Data Reduction and Analysis}

The radius of gyration was calculated according to the Guinier approximation (Glatter \& Kratky, 1981):

$$
\operatorname{LnI}(S)=\operatorname{LnI}(0)-\frac{4 \pi^{2}}{3} R_{\mathrm{g}}{ }^{2} S^{2}
$$

The fitting region is from 0.0045 to $0.01 \AA^{-1}$ in $S$ with $S=2 \sin \theta / \lambda, \lambda$ being $x$-ray wavelength. $\mathrm{I}(0)$, the scattering intensity at zero angle, is proportional to the square of the excess electronic density between the protein molecule and the buffer background. As the urea concentration increases, the contrast in electron density becomes smaller, and I( 0 ) decreases quadratically with urea concentration.

Distance distribution $(\mathrm{P}(\mathrm{r}))$ functions

$$
P(r)=8 \pi r \int_{0}^{\infty} S I(S) \sin (2 \pi S r) d S
$$

were evaluated using the indirect transformation method implemented in the program GNOM (Semenyuk \& Svergun, 1991). The $P(r)$ function measures the density of intramolecular pair distance distributions within the scatterer, the protein molecule. The range used for the $\mathrm{P}(\mathrm{r})$ calculation was from 0.003 to $0.03 \AA^{-1}$ in $\mathrm{S}$. A scattering curve was computed from the coordinates of the crystal structure of lysozyme (entry PDB2LYZ.ENT in Brookhaven Protein Data Bank) using the program CRYSOL (Svergun, et al., 1995). The thickness of the hydration layer was set to one layer of $\mathrm{H}_{2} \mathrm{O}$ molecules, and the excess electron density between this hydration layer and the buffer was taken to be $0.03 \mathrm{e} / \AA^{3}$. 


\subsubsection{Thermodynamic Analysis of the Denaturation Curve}

The denaturation curve was analyzed with either a two-state or a three-state model. The following analysis was based on the three-state analysis, and could easily be applied to the two-state model. For an equilibrium ternary system consisting of native $(N)$, intermediate $(\mathrm{I})$ and unfolded $(\mathrm{U})$ molecules, the measured amide $\mathrm{CD}$ signals $(\theta)$ and radius of gyration $\left(\mathrm{R}_{\mathrm{g}}\right)$ were decomposed according to equations [3] and [4]:

$$
\begin{aligned}
& \theta^{k}=f_{N}^{k} \theta_{N}+f_{I}^{k} \theta_{I}+f_{U}^{k} \theta_{U} \\
& R_{g, k}^{2}=f_{N}^{k} R_{g, N}^{2}+f_{I}^{k} R_{g, I}^{2}+f_{U}^{k} R_{g, U}^{2}
\end{aligned}
$$

where $f_{N}^{k}, f_{1}^{k}$ and $f_{U}^{k}$ are the fractions of the protein molecules in the native, intermediate and unfolded states respectively. At equilibrium, the fractions can be derived thermodynamically:

$$
\begin{aligned}
& f_{N}^{k}=\frac{1}{1+K_{N \rightarrow I}^{k}+K_{N->U}^{k}} \\
& f_{I}^{k}=\frac{K_{N \rightarrow 1}^{k}}{1+K_{N->I}^{k}+K_{N \rightarrow U}^{k}} \\
& f_{U}^{k}=\frac{K_{N \rightarrow U}^{k}}{1+K_{N \rightarrow I}^{k}+K_{N}^{k}>U}
\end{aligned}
$$

where $\mathrm{K}_{\mathrm{N} \rightarrow \mathrm{I}}^{\mathrm{k}}$ and $\mathrm{K}_{\mathrm{N} \rightarrow \mathrm{U}}^{\mathrm{k}}$ are the equilibrium constants for native $\rightarrow$ intermediate and native $\rightarrow$ unfolded transitions at $k M$ urea solution respectively; and $f_{N}^{k}+f_{1}^{k}+f_{U}^{k}=1$. The equilibrium constants are related to the changes in Gibbs energy as:

$$
\begin{aligned}
& \Delta \mathrm{G}_{\mathrm{I}}^{\mathrm{k}}=-\mathrm{RT} \ln \mathrm{K}_{\mathrm{N}->\mathrm{I}}^{\mathrm{k}} \\
& \Delta \mathrm{G}_{\mathrm{U}}^{\mathrm{k}}=-\mathrm{RT} \ln \mathrm{K}_{\mathrm{N} \rightarrow \mathrm{U}}^{\mathrm{k}}
\end{aligned}
$$




\subsubsection{Models of Denaturant Effects}

$\Delta \mathrm{G}_{\mathrm{U}}^{\mathrm{H}_{2} \mathrm{O}}$, the free energy difference between the native and the unfolded state of a protein extroplated to zero denaturant concentration, is a good indication of the stability of a native protein. Estimates of $\Delta \mathrm{G}_{\mathrm{U}}^{\mathrm{H}_{2} \mathrm{O}}$ come from calorimetic measurements, hydrogen exchange studies, and mostly from analysis of the denaturation curve (Pace, 1986). To analyze the denaturation curve, three models are used to account for the effect of denaturants on protein stability.

Tanford's model. This model attributes the unfolding ability of denaturants to an increase in solubility of the constituent parts of proteins with increasing denaturant concentration (Tanford, 1970). Therefore, free energies of transfer between $\mathrm{H}_{2} \mathrm{O}$ and denaturant (urea or $\mathrm{GdmHCl}$ ), derived from solubility studies on amino acids and peptides, can be used quantitatively to describe unfolding of proteins by denaturants:

$$
\Delta G_{U}=\Delta G_{U}^{H_{2} O}+\bar{\alpha} \sum_{i} n_{j} \delta g_{t r, i}
$$

where $\delta g_{t r, i}$ is the free energy for transfer of a group of type $i$ from $\mathrm{H}_{2} \mathrm{O}$ to denaturant, $\bar{\alpha}$ is the average fractional change in the degree of exposure for all groups when a protein unfolds, and $n_{\mathrm{i}}$ is the number of groups of type $i$ in the protein. Values of both $\delta g_{t r, i}$ and $n_{\mathrm{j}}$ are available in the literature. $\bar{\alpha}$ is chosen so that the dependence of $\Delta \mathrm{G}_{\mathrm{U}}$ on denaturant concentration calcuated from equation [7] is equal to the experimentally derived value, the $m$ value in equation [8] (see below).

Linear extrapolation model. This simple model (Greene \& Pace, 1974) assumes that $\Delta \mathrm{G}_{\mathrm{U}}^{\mathrm{k}}$ is linearly dependent on denaturant concentration, and that an estimate of $\Delta \mathrm{G}_{\mathrm{U}}^{\mathrm{H}_{2} \mathrm{O}}$ can be obtained by extrapolation of $\Delta \mathrm{G}_{U}^{\mathrm{k}}$ to zero denaturant concentration:

$$
\Delta \mathrm{G}_{\mathrm{U}}^{\mathrm{k}}=\Delta \mathrm{G}_{\mathrm{U}}^{\mathrm{H}_{2} \mathrm{O}}-\mathrm{m}[\text { denaturant }]
$$

The assumption is based on the observation of the linear dependence of $\Delta \mathrm{G}_{\mathrm{U}}^{\mathrm{k}}$ on denaturant concentration in the transition region. This method normally gives a lower value of $\Delta \mathrm{G}_{\mathrm{U}}^{\mathrm{H}_{2} \mathrm{O}}$ than the other two models described herein. 
Denaturant binding model. This model is employed in our study to account for the effect of urea on protein stability. The model assumes that unfolding of a protein results from an increase in the number of denaturant binding sites on the unfolded (or intermediate) conformation compared to the native form (Aune \& Tanford, 1969):

$$
\begin{aligned}
& \Delta G_{I}^{k}=\Delta G_{I}^{H_{2} \mathrm{O}}-\Delta n_{I} R T \ln (1+c a) \\
& \Delta G_{U}^{k}=\Delta G_{U}^{H_{2} O}-\Delta n_{U} R T \ln (1+c a)
\end{aligned}
$$

where $\Delta \mathrm{G}_{1}^{\mathrm{H}_{2} \mathrm{O}}$ and $\Delta \mathrm{G}_{\mathrm{U}}^{\mathrm{H}_{2} \mathrm{O}}$ are the changes in free energy in the unfolding of the native to the intermediate and the native to the unfolded states respectively, extrapolated to zero denaturant concentration. $\Delta n_{1}$ and $\Delta n_{U}$ are the differences in the number of urea binding sites between the native and the intermediate states, and between the native and the unfolded states. $\mathrm{R}$ is the gas constant, and $\mathrm{T}$ is the temperature. $\mathrm{c}$ is the averaged equilibrium binding constant at each site. $a$ is the activity of urea which is related to the molar concentration, $\mathrm{M}$, by the relation: $\mathrm{a}=0.9815(\mathrm{M})-0.02978(\mathrm{M})^{2}+0.00308(\mathrm{M})^{3}$ (Pace, 1986).

Substitutions of the terms in equations [3] and [4] with corresponding terms from equations [5a-c], [6a-b] and [9a-b] give the following two equations:

$$
\begin{aligned}
& \theta^{k}=\frac{\left(\theta_{N}+\theta_{\mathrm{I}} \mathrm{e}^{-\gamma}+\theta_{\mathrm{U}} \mathrm{e}^{-\rho}\right)}{1+\mathrm{e}^{-\gamma}+\mathrm{e}^{-\rho}} \\
& \mathrm{R}_{\mathrm{g}, \mathrm{k}}^{2}=\frac{\left(\mathrm{R}_{\mathrm{g}, \mathrm{N}}^{2}+\mathrm{R}_{\mathrm{g}, \mathrm{I}}^{2} \mathrm{e}^{-\gamma}+\mathrm{R}_{\mathrm{g}, \mathrm{U}}^{2} \mathrm{e}^{-\rho}\right)}{1+\mathrm{e}^{-\gamma}+\mathrm{e}^{-\rho}}
\end{aligned}
$$

where

$$
\begin{aligned}
& \gamma=\left(\Delta G_{I}^{\mathrm{H}_{2} \mathrm{O}}-\Delta \mathrm{n}_{\mathrm{I}} \mathrm{RT} \ln (1+\mathrm{ca})\right) / \mathrm{RT} \\
& \rho=\left(\Delta \mathrm{G}_{\mathrm{U}}^{\mathrm{H}_{2} \mathrm{O}}-\Delta \mathrm{n}_{\mathrm{U}} \mathrm{RT} \ln (1+\mathrm{ca})\right) / \mathrm{RT}
\end{aligned}
$$

These two equations are used for simultaneous fit of the $\mathrm{CD}$ and $\mathrm{R}_{\mathrm{g}}$ data using a nonlinear least squares method. 


\subsubsection{Singular Value Decomposition (SVD)}

Singular value decomposition (SVD) (Golub \& Reinsch, 1970; Press, et al., 1992) has been successfully applied to the analysis of transient absorption spectra to resolve and identify kinetic intermediates involved in the process of photodissociation of carbon monoxide in hemoglobin and myoglobin (Hofrichter, et al., 1983; Lambright, et al., 1991). The method has also been utilized in X-ray scattering studies of structural transition of ATCase (Fetler, et al., 1995). The significance of this technique is two-fold (Shrager \& Hendler, 1982): 1) It does not require a prior knowledge of the models, i.e. the scattering curves of the components; 2) It is particularly powerful in decomposing complex spectra where components share overlapping features.

To determine the minimum number of basis scattering functions in the unfolding transition, the SVD method was applied to the analysis of the scattering curves, based on the supposition that the scattering curves are very likely to be of highly singular value. Unlike the $\mathrm{R}_{\mathrm{g}}$ analysis in which only the scattering data from the small angle (Guinier) region is used, SVD is applied to the whole scattering space, including the Guinier region. The application of SVD to the analysis of titration data has been described (Fetler, et al., 1995; Provencher \& Blockner, 1983; Shrager \& Hendler, 1982), thus only the salient features are summarized here.

A set of scattering profiles measured for a range of denaturant concentrations can be represented as an $\mathrm{m} \times \mathrm{n}$ matrix $A(\mathrm{~S}, \mathrm{k})$, with each column being the scattering profiles at $\mathrm{k}$ $M$ urea concentration. According to the therom of SVD, any matrix may be written as the product of three matrices:

$$
A(\mathrm{~S}, \mathrm{k})=U(\mathrm{~S}, \mathrm{k}) S V^{\mathrm{T}}
$$

where $U(\mathrm{~S}, \mathrm{k})$ is an $\mathrm{m} \times \mathrm{n}$ matrix of orthogonal columns which form a complete set of basis scattering curves in terms of which the scattering spectrum at any urea concentration can be represented as a linear superposition. The column of $U(S, k)$ with the largest singular value is the best single-component representation of the $A(\mathrm{~S}, \mathrm{k})$ in the least square sense. The first two columns constitute the best two-component representation $A(\mathrm{~S}, \mathrm{k})$ and so on. $S$ is an $\mathrm{n} \times \mathrm{n}$ diagonal matrix with non-negative elements (w) called the singular values, which describe the overall contribution of each basis scattering curve $U(\mathrm{~S}, \mathrm{k}) . V^{\mathrm{T}}$ 
is the transpose of an $\mathrm{n} \times \mathrm{n}$ orthonormal matrix $V$, whose columns represent the denaturant concentration depedence for each of the basic scattering curve. Each row of $S V^{\mathrm{T}}$ contains the set of urea-dependent linear superposition coefficients corresponding to each basis function.

The tremendous utility of SVD is realized when only a few components are found to be significant. In the present application, it is expected that only a few basic scattering functions will make significant contributions. The remains have singular values so close to zero that their contributions do not add to our structural knowledge, so they are neglected. The scattering curve at $\mathbf{k} \mathrm{M}$ denaturant can then be approximated as a linear combination of the significant basis functions:

$$
a_{k}(S)=\sum_{j=1}^{L} w_{j} V_{k j} U_{j}
$$

where $L$ is the minimum number of components that are required to represent each of the scattering curves, terms with $\mathrm{j}>\mathrm{L}$ representing noise. $a_{k}(\mathrm{~S})$, the column of matrix $A(\mathrm{~S}$, $\mathrm{k}$ ), represents the scattering intensity at $\mathrm{kM}$ urea, $\mathrm{w}_{\mathrm{j}}$ is the singular value of the basis function $\mathrm{U}_{\mathrm{j}}$, and $\mathrm{V}_{\mathrm{kj}}$ contains denaturant-dependent coefficients of the basis function $\mathrm{U}_{\mathrm{j}}$ at k M urea.

It should be noted that the significant basis functions (or scattering curves) do not represent the scattering curves of molecular species. However, SVD does resolve the number of scattering distinct species $(L)$ by determining the number of significant singular values contained in the data.

Background subtracted scattering data were first normalized to the same $I(0)$ as with the native condition, in $0 \mathrm{M}$ urea, to account for the decrease in electron density contrast due to the increase in urea concentration. Data were presented in $S^{2} I$ vs $S$ (Kratky form) and then smoothed with a three-point polynomial. The number of significant basis functions were selected based on the four criteria: significance of the singular value, the autocorrelation of the corresponding column $U$, the shape of the basis function, and the minimum fitting reduced-chi-square $\left(\bar{\chi}^{2}\right)$. 


$$
\begin{aligned}
\bar{\chi}^{2} & =\frac{1}{m \times(n-j)} \sum_{k} \sum_{i=1}^{m}\left(\frac{S_{i}^{2} \times I_{\text {exp }}^{k}\left(S_{i}\right)-S_{i}^{2} \times I_{c a l, p}^{k}\left(S_{i}\right)}{S_{i}^{2} \times \sigma_{i}}\right)^{2} \\
& =\frac{1}{m \times(n-j)} \sum_{k} \sum_{i=1}^{m}\left(\frac{I_{\text {exp }}^{k}\left(S_{i}\right)-I_{c a l, p}^{k}\left(S_{i}\right)}{\sigma_{i}}\right)^{2}
\end{aligned}
$$

where $\sigma_{j}$ is the error associated with the counting statistics at $S_{i}$.

Since the scattering intensity is additive, the scattering intensity ( $\left.\mathrm{I}^{\mathrm{k}}\right)$ of a solution at $\mathrm{k} M$ denaturant concentration with three species, native (N), intermediate (I) and unfolded (U) can be expressed as:

$$
S^{2} I^{k}=f_{N}^{k} S^{2} I_{N}+f_{I}^{k} S^{2} I_{I}+f_{U}^{k} S^{2} I_{U}
$$

where $f_{N}^{k}, f_{1}^{k}$ and $f_{U}^{k}$ are fractions of native (0 M urea), intermediate and unfolded (8M urea) species at $\mathrm{kM}$ urea solution respectively, and can be expressed as in equations [5a] to [5c]. According to SVD, the scattering curves can be approximated by the linear combination of the basis functions, so that equation [14] can be rewritten as:

$$
\begin{aligned}
S^{2} I^{k}= & f_{N}^{k}\left(b_{1}^{N} U_{1}+b_{2}^{N} U_{2}+b_{3}^{N} U_{3}\right)+f_{1}^{k}\left(b_{1}^{I} U_{1}+b_{2}^{I} U_{2}+b_{3}^{I} U_{3}\right) \\
& +f_{U}^{k}\left(b_{1}^{U} U_{1}+b_{2}^{U} U_{2}+b_{3}^{U} U_{3}\right)
\end{aligned}
$$

where $b_{i}^{j}(i=1,2,3$, and $j$ denotes $N, I, U$ for native, intermediate, and unfolded states, respectively) are the coefficients for $U_{1}, U_{2}$, and $U_{3}$ respectively. On the other hand, the SVD approximation of $S^{2} I^{k}$ is:

$$
S^{2} I^{k}=b_{1}^{k} U_{1}+b_{2}^{k} U_{2}+b_{3}^{k} U_{3}
$$

where $b_{1}^{k}, b_{2}^{k}$ and $b_{3}^{k}$ are the coefficients for $U_{1}, U_{2}$, and $U_{3}$ at $k M$ urea concentration, derived from columns of $S V$. Since $\mathrm{U}_{1}, \mathrm{U}_{2}, \mathrm{U}_{3}$ are linearly independent, the following equations are obtained by comparing equations [15] and [16]:

$$
\begin{aligned}
& b_{1}^{k}=f_{N}^{k} b_{1}^{N}+f_{1}^{k} b_{1}^{I}+f_{U}^{k} b_{1}^{U} \\
& b_{2}^{k}=f_{N}^{k} b_{2}^{N}+f_{I}^{k} b_{2}^{I}+f_{U}^{k} b_{2}^{U}
\end{aligned}
$$




$$
b_{3}^{k}=f_{N}^{k} b_{3}^{N}+f_{1}^{k} b_{3}^{I}+f_{U}^{k} b_{3}^{U}
$$

Now, $b_{1}^{I}, b_{2}^{I}$ and $b_{3}^{I}$, the coefficients of the intermediate, can be calculated by minimizing the following chi-square:

$$
\chi^{2}=\sum_{k} \sum_{i=1}^{L}\left(b_{i, o b s}^{k}-b_{i, c a l}^{k}\right)^{2}
$$

The scattering curves of the intermediate can be constructed from the basis functions $\left(\mathrm{U}_{1}, \mathrm{U}_{2}, \mathrm{U}_{3}\right)$ and their corresponding coefficients $\left(b_{1}^{\mathrm{I}}, b_{2}^{\mathrm{I}}\right.$ and $\left.b_{3}^{\mathrm{I}}\right)$.

\subsection{Results}

\subsubsection{Sample Integrity}

All data were normalized by the total flux of the incident $x$-ray beam on the cell, followed by background subtraction. To inspect radiation-induced sample aggregation during data collection, scattering curves from all the cycles ( 1 or 2 minutes per cycle) were overlaid and visually examined. A persistent growth in the scattering intensity was observed in the small angle range with the exposure time $>15$ minutes with the monochromatic beam, or $>1$ minute with the multilayer beam ( $\sim 10$ times more intense than the monochromatic beam). The native state was found to be more sensitive to the $\mathrm{x}$-ray radiation than the denatured states. Therefore, for the monochromatic beam, data collection time was limited to 10 minutes; for the multilayer beam, in addition to a short data collection time, aluminum foils were used to attenuate the incident $\mathrm{x}$-rays, and more recently, the solution was flowed continuously through the observation cell to minimize exposure of lysozyme to $\mathrm{x}$-rays.

The overlapping scattering curves from different cycles were then averaged, and calibrated. None of the samples showed upward curvature at the smallest angular region available in the Guinier plot, indicating that the samples were largely homogenous and free of associative components (dimer or higher oligomers) in the solution. For the native state, measurements were carried out over a protein concentration series (from $3 \mathrm{mg} / \mathrm{l}$ to 44 
$\mathrm{mg} / \mathrm{ml}$ ), and the concentration-normalized forward scattering intensity $(\mathrm{l}(0) / \mathrm{C})$ was found to decrease reasonably linearly with protein concentration. In our SAXS experiments, lysozyme is routinely used as a standard protein to estimate the molecular weight $\left(\mathrm{M}_{\mathrm{r}}\right)$ of other proteins studied, such as heat shock proteins (Section 3.4.1.2), according to the following:

$$
\frac{(\mathrm{I}(0) / \mathrm{C})_{\mathrm{C} \rightarrow 0, \text { protein }}}{(\mathrm{I}(0) / \mathrm{C})_{C \rightarrow 0, \text { lysozyme }}}=\frac{\mathrm{MW}_{\text {protein }}}{M W_{\text {lysozyme }}}
$$

where $(\mathrm{I}(0) / \mathrm{C})_{\mathrm{C} \rightarrow 0}$ is the extrapolation of $(\mathrm{I}(0) / \mathrm{C})$ to infinitely dilute protein solution.

\subsubsection{The Native State}

The native state of lysozyme appears to be very stable over the $\mathrm{pH}$ range 1.2-11.3 (Imoto, et al., 1972). However, lysozyme undergoes reversible self-association at $\mathrm{pH}>$ 4.5 with dimers being the predominant species (Bruzzesi, et al., 1965). pH 3.8 of $\mathrm{NaCl}-$ $\mathrm{NaOAc}$ buffer has been commonly used to ensure a homogeneous monomeric lysozyme solution.

Fig. 4.3 shows the concentration dependence of $\mathrm{R}_{\mathrm{g}}$ for lysozyme at $\mathrm{pH} 3.8$. The extrapolated (to zero protein concentration) $R_{g}(15.4 \pm 0.3 \AA$ ) value is within the range of the published results (15-16 $\AA$ ) (Imoto, et al., 1972). Although interparticle interference decreases scattering intensity at the small angle region, resulting in a reduced apparent $R_{g}$ at solutions other than infinite dilution, the effect is not that significant at relatively dilute solutions: a decrease in $\mathrm{R}_{\mathrm{g}}$ of $\sim 3 \%$ at $6 \mathrm{mg} / \mathrm{ml}$ or $-4.5 \%$ at $8 \mathrm{mg} / \mathrm{ml}$ solutions. Because of this, in the equilibrium denaturation and kinetic refolding experiments, described in Chapter 5 , measurements were carried out using lysozyme solutions of $6-8 \mathrm{mg} / \mathrm{ml}$ to improve data statistics.

The negligible interparticle interference effect at lysozyme concentrations lower than $8 \mathrm{mg} / \mathrm{ml}$ can be also seen from Fig. 4.4. At higher protein concentrations, such as 20 $\mathrm{mg} / \mathrm{ml}$ or $44 \mathrm{mg} / \mathrm{ml}$, a depressive trend is seen at the small angle region, due to the strong interparticle interference. The interference appears not to affect the scattering profile at the angular range $S>0.015 \AA^{-1}$. Hence, $0.015 \AA^{-1}$ was used as the merging point where data 


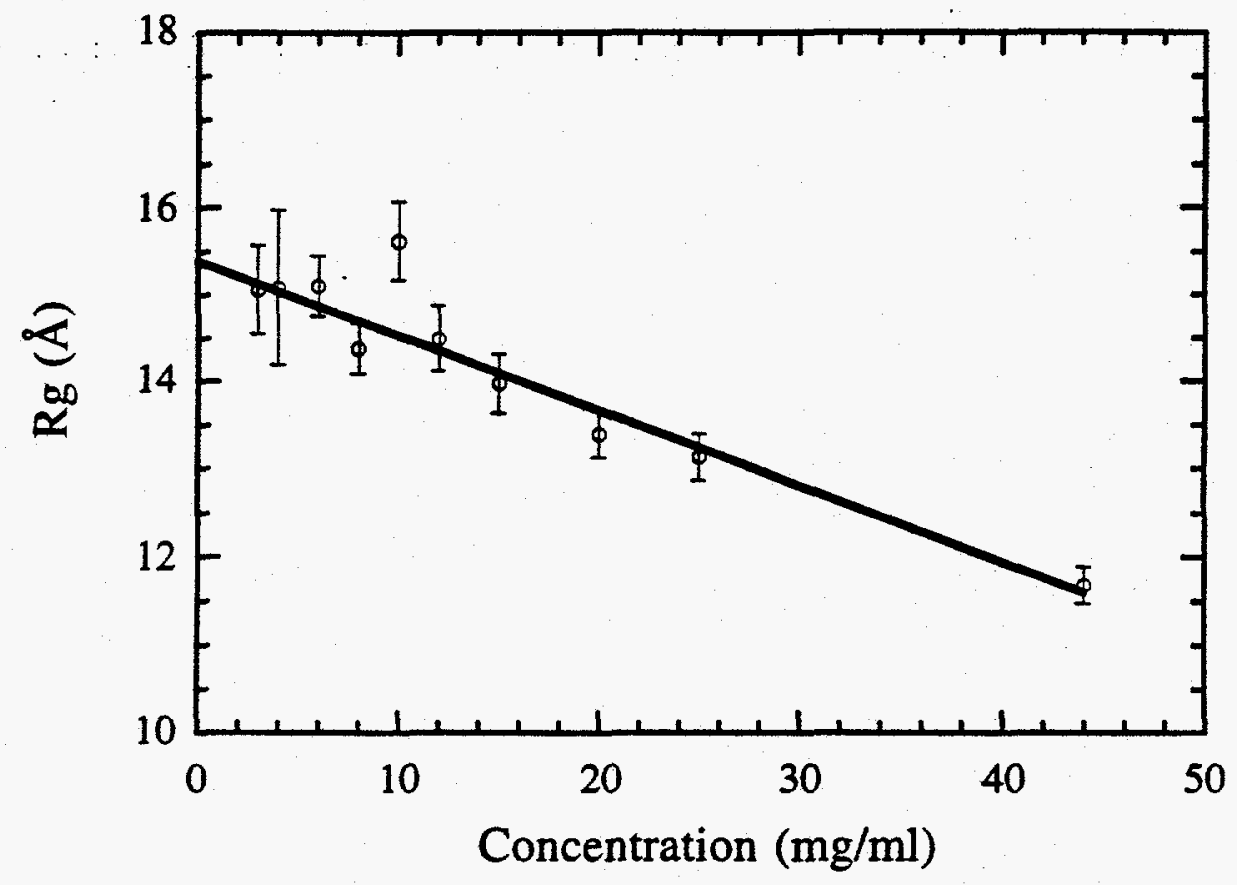

Figure 4.3. Concentration dependence of $\mathrm{R}_{\mathrm{g}}$ of the native lysozyme at $\mathrm{pH} 3.8$, and the extrapolation of $\mathrm{R}_{\mathrm{g}}$ to zero protein concentration. Multiple measurements were carried out for each lysozyme concentration. The error bars represent the maximum value between the standard deviation of different measurements at the same lysozyme concentration and the fitting errors associated with each measurement derived from the Guinier fitting. 


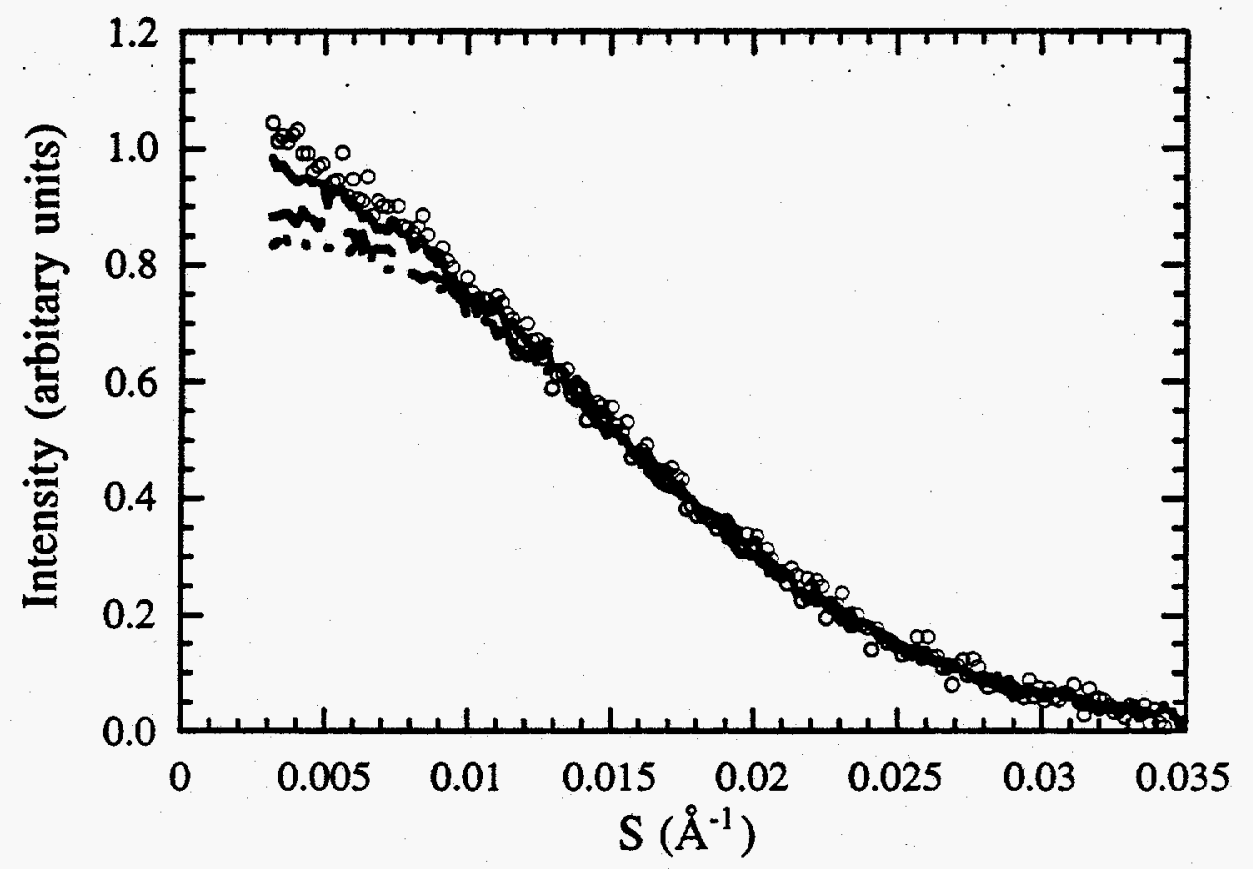

Figure 4.4. Concentration-normalized background-subtracted SAXS data for the native lysozyme at concentrations of $4 \mathrm{mg} / \mathrm{ml}(0), 8 \mathrm{mg} / \mathrm{ml} \mathrm{(-),20} \mathrm{mg/ml} \mathrm{(-} \mathrm{)} \mathrm{and} 44 \mathrm{mg} / \mathrm{ml}$ (- -). Interparticle interference effect is not significant below $8 \mathrm{mg} / \mathrm{ml}$. All four scattering curves overlap at angular range $S>0.015 \AA^{-1}$, suggesting that interparticle effect can be neglected in the wide angle region. 
of the low angular region from the dilute sample $(4 \mathrm{mg} / \mathrm{ml})$ overlapped with data of the wide angular region from concentrated solutions $(20 \mathrm{mg} / \mathrm{ml}$ or $44 \mathrm{mg} / \mathrm{ml})$, to calculate the $P(r)$ function for the native state.

Fig. 4.5 shows the $P(r)$ functions calculated from the $x$-ray solution scattering data and from crystal structure coordinates. The shape of the $P(r)$ function computed from coordinates deviates substantially from that derived from the experiemental data: the $P(r)$ function shifts to smaller distances with the peak positioned at $\sim 17 \AA$, about $1 \AA$ shorter than the experimental $P(r)$ function. The maximum dimension of lysozyme, $D_{\max }$, where $P(r)$ reaches zero, in the crystal $(42 \pm 2 \AA)$ is also smaller than that in solution $(50 \pm 2 \AA)$. When a hydration layer of $3 \AA$ was added around lysozyme, the calculated $P(r)$ function became superimposable with the experimental $P(r)$ function. A hydration layer was also found necessary for the ATPase domain of hsc70 (Section 3.4.3.1) to match the calculated $P(r)$ function with that from $x$-ray solution scattering. The scattering curve thus calculated is in good agreement with the experimental data, as shown in the Kratky plots, Fig. 4.6. The well-defined peak in the Kratky plot indicates that the native lysozyme is a globular molecule in both solution and crystalline forms. Hence, by $\mathrm{x}$-ray scattering technique, lysozyme seems to retain similar conformation in solution and in crystalline form.

Due to its unusual stability, unfolding of lysozyme by urea has to be conducted in reduced $\mathrm{pH}$ solutions. In our equilibrium denaturation and kinetic refolding studies, $\mathrm{pH}$ was reduced to $\mathrm{pH} 2.9$ or $\mathrm{pH} 2.0$, with Na-citrate $\left(\mathrm{NaC}_{6} \mathrm{H}_{7} \mathrm{O}_{6}\right)$ and $\mathrm{Na}$-phosphate $\left(\mathrm{NaH}_{2} \mathrm{PO}_{4}\right)$ replacing the $\mathrm{NaOAc}$ buffer, respectively. Interestingly, at similar lysozyme concentrations, $\mathrm{R}_{\mathrm{g}}$ of lysozyme calculated from these three solutions showed some variations: $15.1 \pm 0.4 \AA$ for $6.0 \mathrm{mg} / \mathrm{ml}$ at $\mathrm{pH} 3.8,15.4 \pm 0.2 \AA$ for $6.8 \mathrm{mg} / \mathrm{ml}$ at $\mathrm{pH} 2.9$, and $14.2 \pm 0.3 \AA$ for $7.3 \mathrm{mg} / \mathrm{ml}$ at pH 2.0. Different counter ions $\left(\mathrm{OAc}^{-}, \mathrm{H}_{2} \mathrm{PO}_{4}^{-}\right.$, $\mathrm{C}_{6} \mathrm{H}_{7} \mathrm{O}_{6}{ }^{\circ}$ ) may be attributed to the observed variations in $\mathrm{R}_{\mathrm{g}}$; in acidic solution, lysozyme carries a net postive charge, for example, a net charge of +18 at $\mathrm{pH} 2.5$ (Bruzzesi, et al., 1965). Batch-to-batch difference of lysozyme sample can not be excluded. The overall scattering curves of these three solutions (Fig. 4.7) overlapped well, suggesting that the conformation of lysozyme in these three solutions was esentially the same, as judged by $x$ ray scattering. 


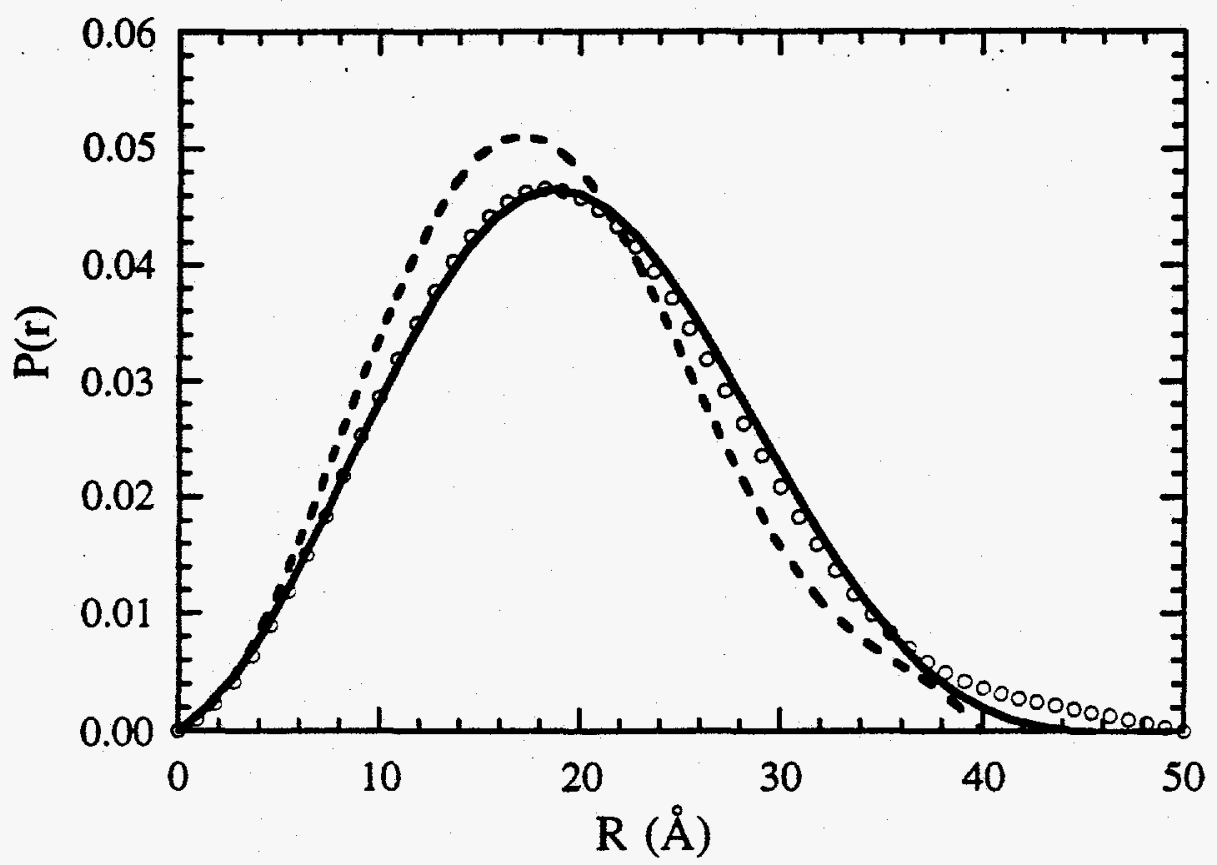

Figure 4.5. Comparisons between the experimental and the calculated $P(r)$ functions of the native lysozyme. (o), derived from SAXS data; (- ), computed from crystal coordinates; (-), computed from crystal coordinates with a $3 \AA$ hydration layer. To calculate the experimental $P(r)$, data of the low angle region $\left(S<0.015 \AA^{-1}\right)$ from a 4 $\mathrm{mg} / \mathrm{ml}$ sample were combined with data of the wide angle region $\left(S>0.015 \AA^{-1}\right)$ from a $44 \mathrm{mg} / \mathrm{ml}$ sample. 


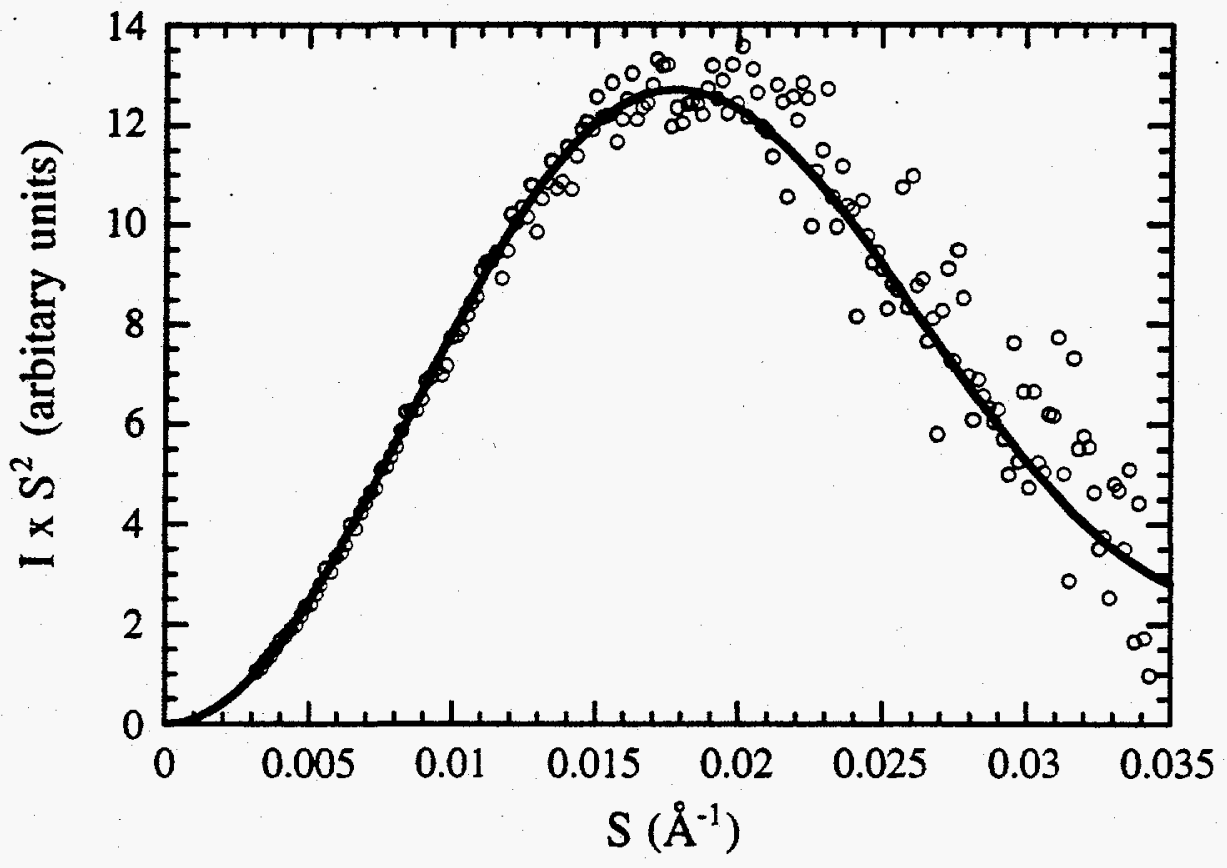

Figure 4.6. Kratky plots of the experimental data of native lysozyme (o), and calculated from crystal structure coordinates with a $3 \AA$ hydration layer added (-). The data set shown is from the sample containing $4 \mathrm{mg} / \mathrm{ml}$ lysozyme. 

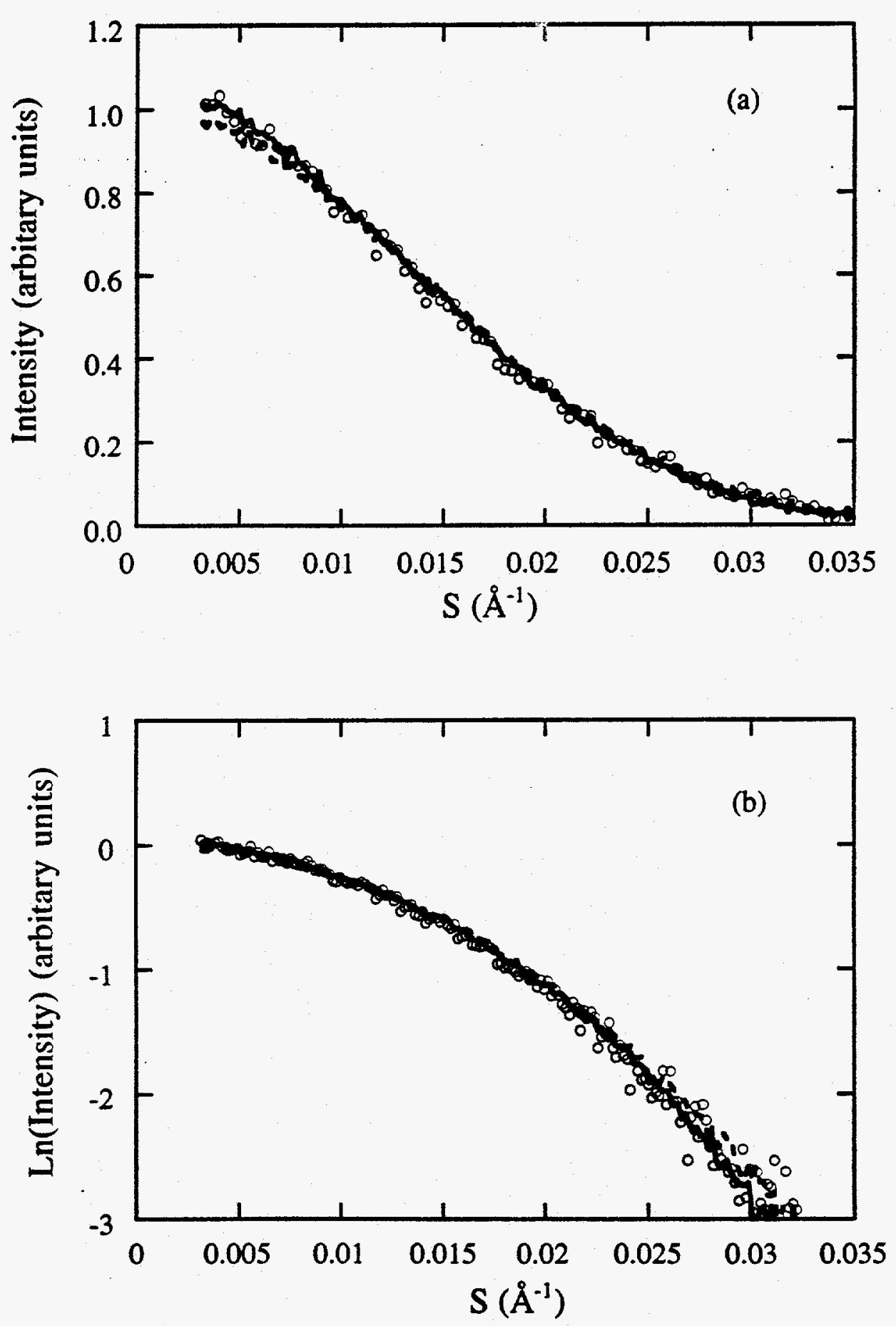

Figure 4.7. Comparisons of scattering curves of the native lysozyme at pH $3.8(0), \mathrm{pH}$ 2.9 (-) and pH $2.0(--)$. (a), in I(S) vs. S plot; (b) in LnI(S) vs. S plot. The differences between these three conditions are negligible. 


\subsubsection{The Unfolded State}

The unfolded states of lysozyme were studied under several conditions: $8 \mathrm{M}$ urea at pH 2.9, $8 \mathrm{M}$ urea at $\mathrm{pH} 2.0,8 \mathrm{M}$ urea and $80 \mathrm{mM}$ reductant, dithiothreitol (DTT) at $\mathrm{pH}$ 2.9 , and 5 or $6 \mathrm{M} \mathrm{GdmHCl}$ at $\mathrm{pH} 2.9$. Suprisingly, conformations of lysozyme in different nonreducing solutions appeared to be similar. For all three conditions ( $8 \mathrm{M}$ urea $\mathrm{pH} 2.0,8 \mathrm{M}$ urea $\mathrm{pH} 2.0$ and $6 \mathrm{M} \mathrm{GdmHCl} \mathrm{pH} 2.9$ ), $\mathrm{R}_{\mathrm{g}}$ was found to be around $22 \AA$, and scattering profiles were superimposible (data not shown). An addition of reductant, DTT, decreased the compactness of unfolded lysozyme by an increase of $\sim 2 \AA$ in $R_{g}$ resulting in a $R_{g}$ of $\sim 24 \AA$.

Heparin, a sulfated mucopolysaccharide, was used as a model for a random coil (Liberti \& Stivala, 1967). Heparin is a particularly good model for comparison with lysozyme because it has a similar molecular weight $(\sim 13 k)$ to that of lysozyme. Heparin has an $\mathrm{Rg}_{\mathrm{g}}$ of $31.3 \pm 0.2 \AA$ by SAXS (Fig. 4.8), much larger than that of unfolded lysozyme, even in the dithiothreitol reduced form. The Kratky plot of heparin (Fig. 4.9.a) shows a plateau at higher angle region, which is a signature of a random coil. However, the Kratky plot of lysozyme in $8 \mathrm{M}$ urea solution appears peaky, suggesting that lysozyme molecules in $8 \mathrm{M}$ urea do not resemble random coils. This is plausible from a structural point of view. Lysozyme has four disulfide bonds distributed over the polypeptide chain (6-127, 30-115, 64-80, 76-94), and they remain intact under the conditions used in our present study, except for solution containing DTT. Structural constraints imposed by these four disulfide bonds restrict the freedom of polypeptide chain movement, resulting in a more compact ensemble of configuration than that expected for a random coil.

The distance distribution $(\mathrm{P}(\mathrm{r})$ ) function for the unfolded state in $8 \mathrm{M}$ urea without reductants is shown in Fig. 4.9.b. $D_{\max }$ is enlarged to $\sim 70 \AA$, and the peak position is shifted to a larger distance, $\sim 25 \AA$, upon unfolding in $8 \mathrm{M}$ urea. As a comparison, $\mathrm{D}_{\max }$ for heparin is $\sim 120 \AA$ (data not shown). The rather smaller $D_{\max }$ again suggests that lysozyme molecules in $8 \mathrm{M}$ urea have some internal structure, and cannot be approximated as a random coil. 


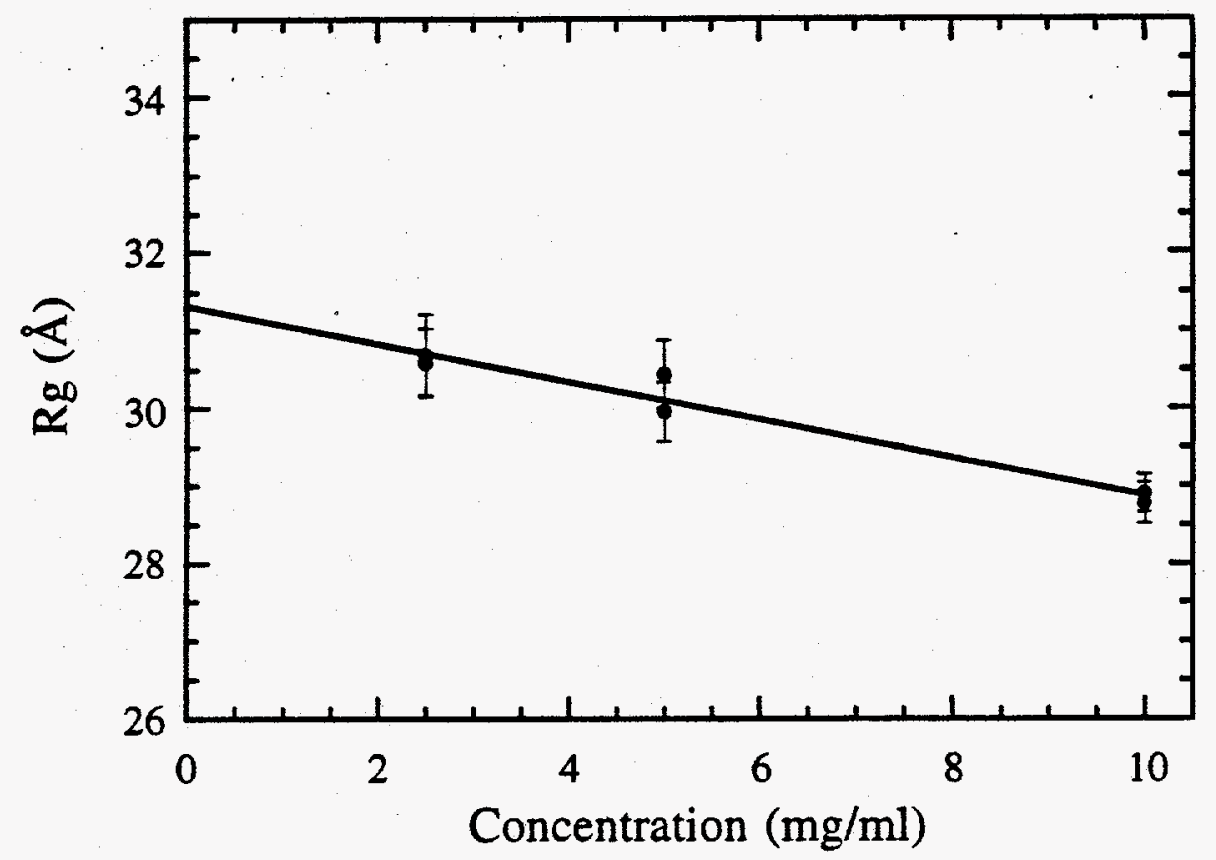

Figure 4.8. Concentration dependence of $\mathrm{Rg}$ of heparin at $\mathrm{pH} 2.5$, and the extrapolation of $R_{g}$ to zero heparin concentration. 

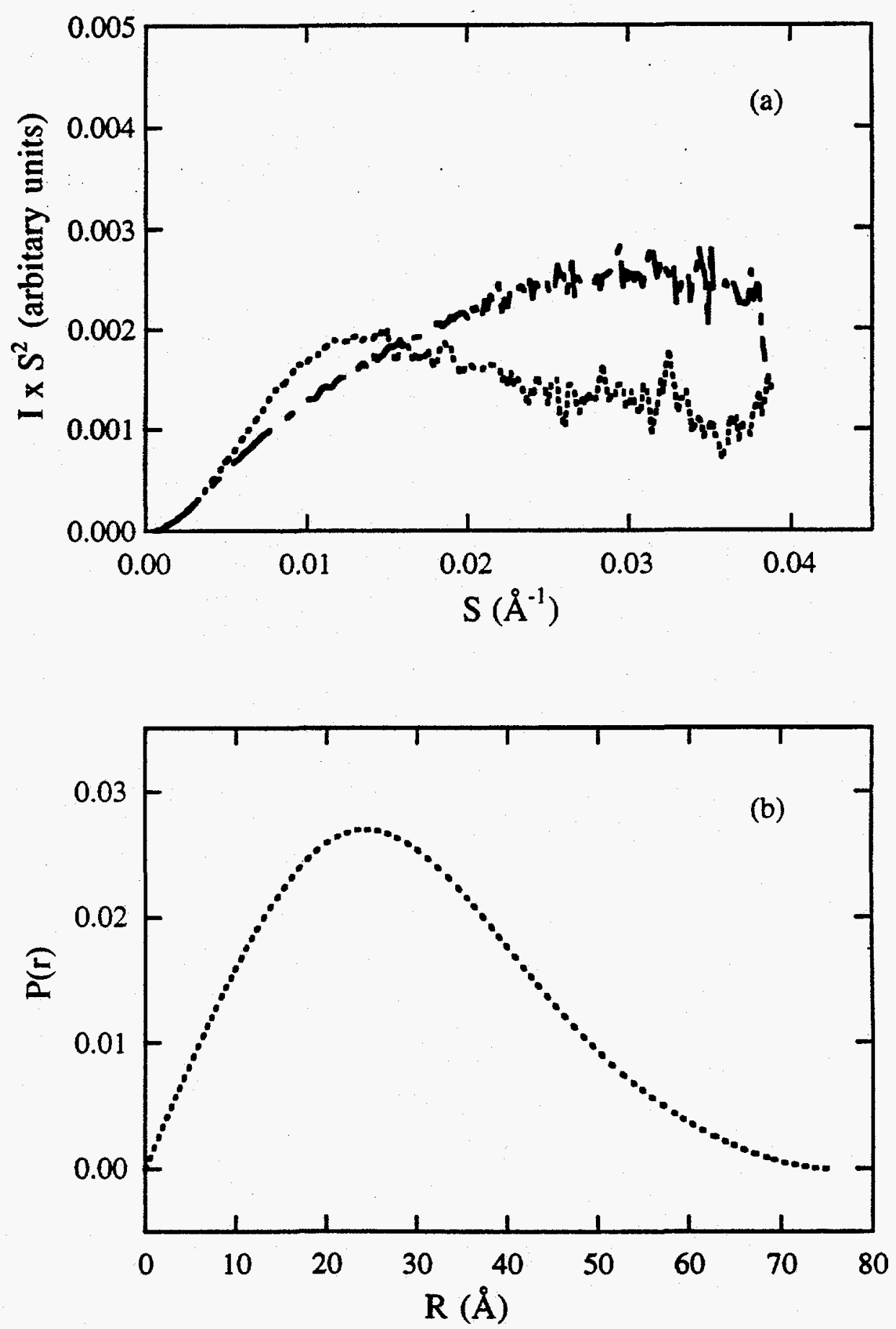

Figure 4.9. a) Kratky plots of a random coil model, heparin, (-- - and lysozyme in 8 $\mathrm{M}$ urea solution $\mathrm{pH} 2.9(--)$. b) The $\mathrm{P}(\mathrm{r})$ function of lysozyme in $8 \mathrm{M}$ urea $\mathrm{pH}$ 2.9. The $\mathrm{P}(\mathrm{r})$ function from the $\mathrm{pH} 2.0$ sample overlaps with that of $\mathrm{pH} 2.9$; data of the $6 \mathrm{M}$ $\mathrm{GdmHCl}$ sample is too noisy to compute a reliable $\mathrm{P}(\mathrm{r})$ function. 


\subsubsection{Unfolding by Urea at $\mathbf{p H} 2.9$}

Like in the native state, a depressive trend at the small angle region was not seen in the Guinier plot, suggesting that the interparticle interference of lysozyme at $-7 \mathrm{mg} / \mathrm{ml}$ over the urea concentations tested was insignificant. The forward scattering intensity is proportional to the squares of both the electronic contrast between the protein molecule and the buffer background and the partial specific volume of the protein: $\mathrm{I}(0) \propto\left(\rho_{\mathrm{p}}-\rho_{\mathrm{b}}\right)^{2} \times \mathrm{V}^{2}$. Since the difference in partial specific volume of lysozyme in $8 \mathrm{M}$ urea $(0.707 \mathrm{ml} / \mathrm{g})$ versus in the native state $(0.702 \mathrm{ml} / \mathrm{g})$ is negligible (Prakash, et al., $1981), \mathrm{I}(0)$ is thus proportional to $\left(\rho_{\mathrm{p}}-\rho_{\mathrm{b}}\right)^{2}$. Fig. 4.10 shows the quadratic decrease in $I(0)$ with the increase of urea concentration. After taking into account the urea effect and normalizing to protein concentration, $\mathrm{I}(0)$ values appear to be rather constant throughout the urea concentrations tested. The similar $\mathrm{I}(0)$ indicates that lysozyme remained unaggregated throughout the folded and unfolded transition.

Unfolding curves determined by amide $\mathbf{C D}$ and $\mathbf{R}_{\mathbf{g}}$. Fig. 4.11.a shows the change of apparent unfolded fraction $\left(\mathrm{F}_{\mathrm{app}}\right)$ of lysozyme with urea concentration at $\mathrm{pH} 2.9$ by $\mathrm{CD}$ and scattering measurements. The apparent fraction is defined as $F_{\text {app }}=\left(Y_{o b s}-Y_{N}\right) /\left(Y_{U}-Y_{N}\right)$, where $Y_{o b s}$ is the experimental value of the physical parameter, $[\theta]_{222}$ or $R_{g}$, at certain urea concentration; $Y_{N}$ and $Y_{U}$ are values of the parameters in the native and unfolded form respectively. For $\mathrm{pH} 2.9$, the transition in the $\mathrm{CD}$ is sharp, suggesting a highly cooperative unfolding process, while the transition in $\mathrm{R}_{\mathrm{g}}$ is more gradual and thus less cooperative. It should be noted that the results of different unfolding behavior observed by amide $C D$ and $R_{g}$ have been reproduced in several independent experimental runs, ruling out the possibility of artifacts. In addition, the difference is clearly resolved at $\mathrm{pH} 2.0$ (see Fig. 4.11.b). Due to limited amounts of beamtime, data at $\mathrm{pH} 2.0$ were not as complete as those at $\mathrm{pH} 2.9$, particularly in the posttransition region, resulting in less conclusive results. Therefore, the following analysis and discussion are focused on data at $\mathrm{pH} 2.9$, and are supported by results at $\mathrm{pH} 2.0$. Different unfolding behavior revealed by these two techniques are due to the fact that they probe different aspects of protein structure: amide $C D$ at $222 \mathrm{~nm}$ mainly reflects the helical content, while $R_{\mathrm{g}}$ measures the size, and hence compactness of the molecule. The differences in the unfolding curves measured by different techniques suggest the presence of intermediate states. The observation that a structural transition measured by $R_{g}$ occurs at lower urea concentration than that by $\mathrm{CD}$, in conjunction with the assumption that $\alpha$-helical 


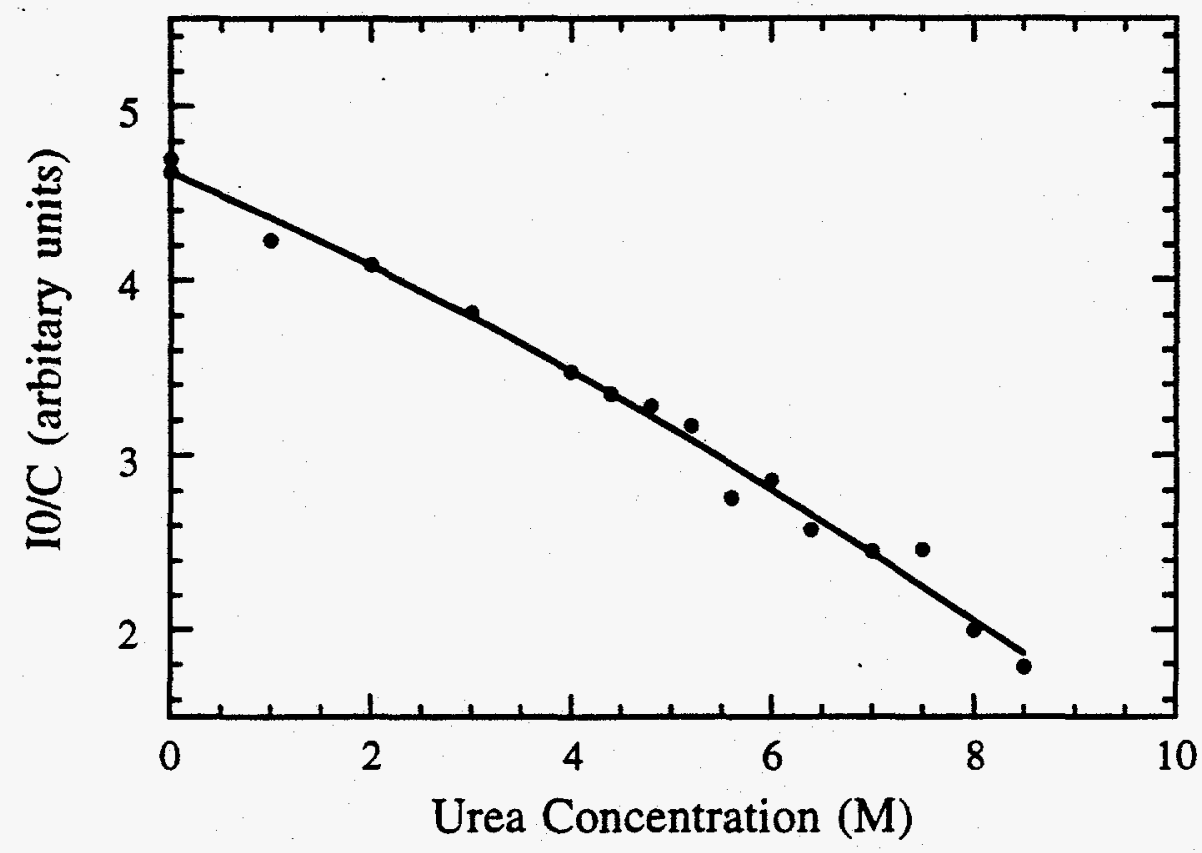

Figure 4.10. The concentration-normalized forward $x$-ray scattering intensity $(I(0) / C)$ of lysozyme as a function of urea concentration at $\mathrm{pH} 2.9$. The line is a second order polynomial fit to the data. 

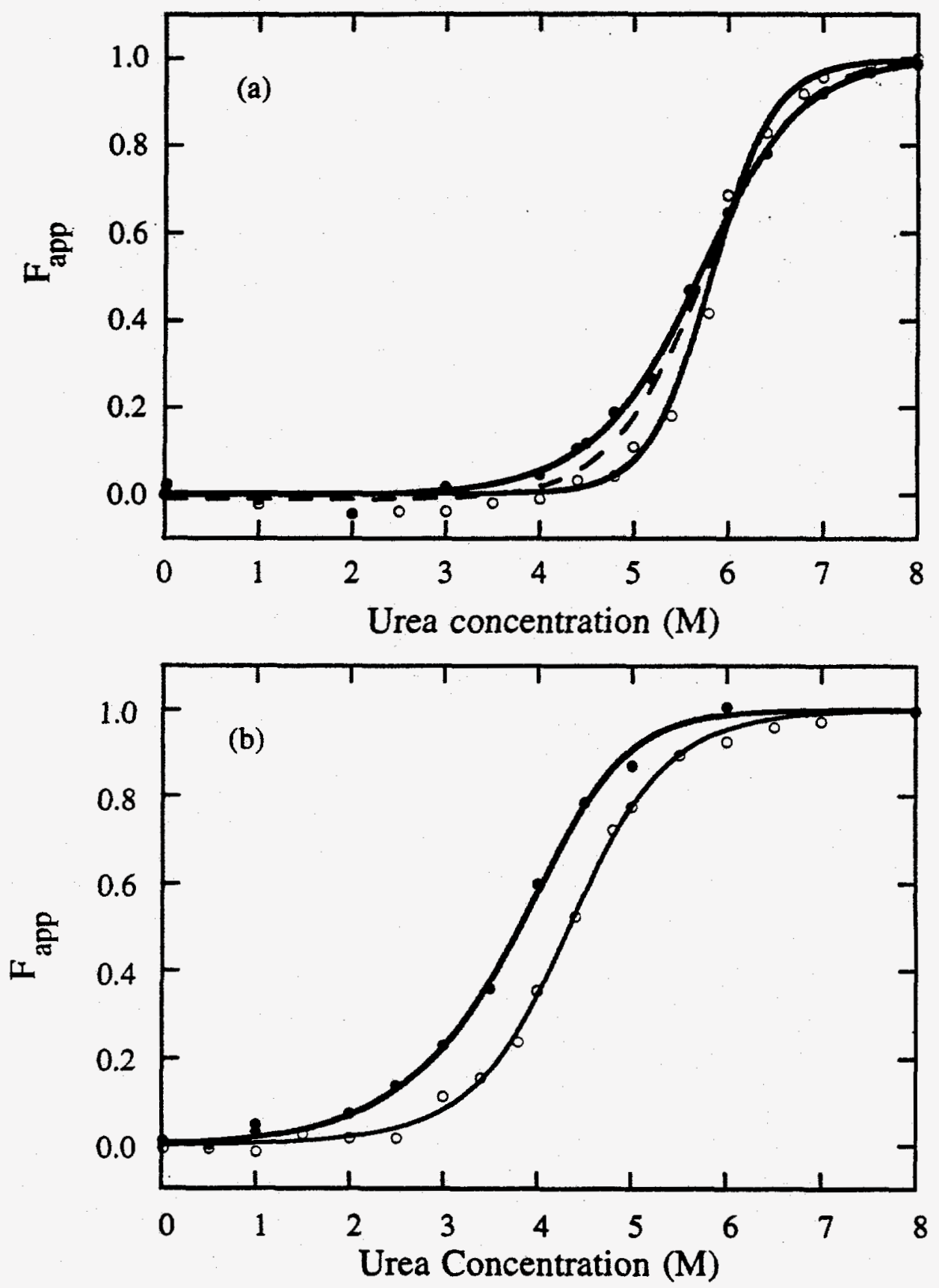

Figure 4.11. Changes of the apparent fraction, $F_{\text {app }}$ (see text for definition), in the ureainduced lysozyme unfolding at $20^{\circ} \mathrm{C}$. $(\bullet)$, by $R_{\mathrm{g}}$ from $\mathrm{x}$-ray scattering; (0), by amide $\mathrm{CD}$ $\theta_{222}$. (a) $\mathrm{pH}$ 2.9. Solid lines are fitting results of scattering and $C D$ data according to model 2 (see text); Dashed lines are the fits to model 1 (see text). The curves from the two sets of fits are almost identical and bence the two dashed lines overlay each other. (b) $\mathrm{pH}$ 2.0. Solid lines are preliminary fitting results according to model 2. 
secondary structure persists in the intermediate state, leads to a working model that the intermediate has an extended $R_{\mathrm{g}}$ but retains native-like $\alpha$ helical content.

To estimate the thermodynamic parameters associated with unfolding process, a denaturant binding model (see Section 4.3.3) was used to account for the effect of urea on the equilibrium constants. A range of different urea binding constants (c) $0.1,0.2,0.4$, $0.6,0.8,1.0$ was tried, and it was found that the fractions of native, intermediate and unfolded states were not affected (within $1 \%$ ) by the $c$ value. The results presented here were from fits with $c$ set to 0.1 (Pace, 1975). Initially, parameters for native, unfolding and intermediate states $\left(R_{g}\right.$ and $\left.\theta\right)$, as well as thermodynamic parameters were not fixed during the fitting. After one round of nonlinear least squares fits, parameters $\left(R_{g}\right.$ and $\left.\theta\right)$ of the native and unfolded states were fixed, the other parameters $\left(\Delta \mathrm{G}_{1}^{\mathrm{H}_{2} \mathrm{O}}, \Delta \mathrm{G}_{\mathrm{U}}^{\mathrm{H}_{2} \mathrm{O}}, \Delta \mathrm{n}_{\mathrm{I}}\right.$, $\Delta \mathrm{n}_{\mathrm{U}}, \mathrm{R}_{\mathrm{g}}^{\mathrm{I}}$ ) were further refined. The refined parameters for the native and the unfolded states were found to be the same as values measured in $0 \mathrm{M}$ and $8 \mathrm{M}$ urea solutions, respectively. Data were simultaneously fit to two models: (1) both transitions in the $C D$ and $R_{g}$ curves taken as two-state; (2) the $C D$ transition assumed to be two-state while the $\mathrm{R}_{\mathrm{g}}$ transition as three-state. The fits are shown in Fig. 4.11.a, and the results are listed in Table 4.1. It is apparent from the figure that the best fit for both $C D$ and $R_{g}$ data is achieved with model (2). $R_{g}$ for the intermediate state is found to be $-19.8 \AA$, as compared to $15.3 \AA$ for the native and $21.9 \AA$ for the unfolded.

Fractions of native, intermediate and unfolded states in different urea concentrations can be calculated by equations [5a-c]. Fig. 4.12 shows the fraction distributions based on $\mathrm{R}_{\mathrm{g}}$. A maximum for the intermediate distribution is found at 5.5-6 M urea concentration, where $\sim 40 \%$ of the lysozyme molecules are partially unfolded. The unfolded species does not appear below $\sim 5 \mathrm{M}$ urea.

SVD analysis. To test the robustness of the SVD method in our studies, several circumstances have been inspected. Firstly, scattering data were also collected at pH 2.0 with the same camera length of $S_{\max }=0.04 \AA^{-1}$, as well as at $\mathrm{pH} 2.9$ but with an extended angular range of $S_{\max }=0.08 \AA^{-1}$. Unfortunately, because of time constraints, experiments under these two situations were not performed in as many urea concentrations as in the case of $\mathrm{pH} 2.9$ with $S_{\max }=0.04 \AA^{-1}$. Nevertheless, three significant basis functions were always observed. Secondly, when a subset of scattering curves (six scattering curves out of a total of 13 curves) at some urea concentrations were selected and subjected to SVD analysis, a third basis function remained evident. Finally, SVD analysis was also carried 
Table 4.1. Parameters for simultaneous fitting model 2 ( $2 \& 3$ state) and model 1 ( $2 \& 2$ state) to the CD ( 222 $\mathrm{nm}$ ) and $\mathrm{R}_{\mathrm{g}}$ unfolding curves in Fig. 4.11.a.

\begin{tabular}{|c|c|c|c|c|c|c|c|}
\hline Model & $\begin{array}{l}\Delta G_{N->I} \\
(\mathrm{kal} / \mathrm{mol})\end{array}$ & $\Delta \mathrm{n}_{\mathrm{N}->\mathrm{I}}$ & $\begin{array}{l}\Delta \mathrm{G}_{\mathrm{N}->\mathrm{U}} \\
(\mathrm{kcal} / \mathrm{mol})\end{array}$ & $\Delta \mathrm{n}_{\mathrm{N}->\mathrm{U}}$ & Native & Intermediate & Unfolded \\
\hline & \multirow{6}{*}{$5.7 \pm 0.3$} & \multirow{6}{*}{$23.6 \pm 1.5$} & \multirow{6}{*}{$11.6 \pm 0.5$} & \multirow{6}{*}{$48.8 \pm 2.1$} & \multicolumn{3}{|c|}{$R_{g}(\AA)$} \\
\hline \multirow[t]{5}{*}{ model (2) } & & & & & $15.3^{\mathrm{a}}$ & $19.8 \pm 0.2^{\mathrm{a}}$ & $21.9^{\mathrm{a}}$ \\
\hline & & & & & $15.3 \pm 0.2^{b}$ & N.A. & $21.8 \pm 0.3^{b}$ \\
\hline & & & & & \multicolumn{3}{|c|}{$\theta_{222}\left(\right.$ mdeg cm$\left.^{2} \mathrm{dmol}^{-1} \mathrm{res}^{-1}\right)$} \\
\hline & & & & & $-8991^{a}$ & N.A. & $-2672^{a}$ \\
\hline & & & & & $-8979^{b}$ & N.A. & $-2660^{b}$ \\
\hline \multirow{6}{*}{ model (1) } & \multirow{6}{*}{ N.A. } & \multirow{6}{*}{ N.A. } & \multirow{6}{*}{$8.0 \pm 0.2$} & \multirow{6}{*}{$32.8 \pm 0.8$} & \multicolumn{3}{|c|}{$\mathrm{Rg}_{\mathrm{g}}(\AA)$} \\
\hline & & & & & $15.5^{\mathrm{a}}$ & N.A. & $21.8^{\mathrm{a}}$ \\
\hline & & & & & $15.3 \pm 0.2^{b}$ & N.A. & $21.8 \pm 0.3^{b}$ \\
\hline & & & & & \multicolumn{3}{|c|}{$\theta_{222}\left(\mathrm{mdeg} \mathrm{cm}^{2} \mathrm{dmol}^{-1} \mathrm{res}^{-1}\right)$} \\
\hline & & & & & $-9173^{a}$ & N.A. & $-2538^{a}$ \\
\hline & & & & & $-8979^{b}$ & & $-2660^{b}$ \\
\hline
\end{tabular}

a used in the denaturation curve fitting.

$\mathrm{b}$ derived from $\mathrm{x}$-ray scattering or $\mathrm{CD}$ measurements. 


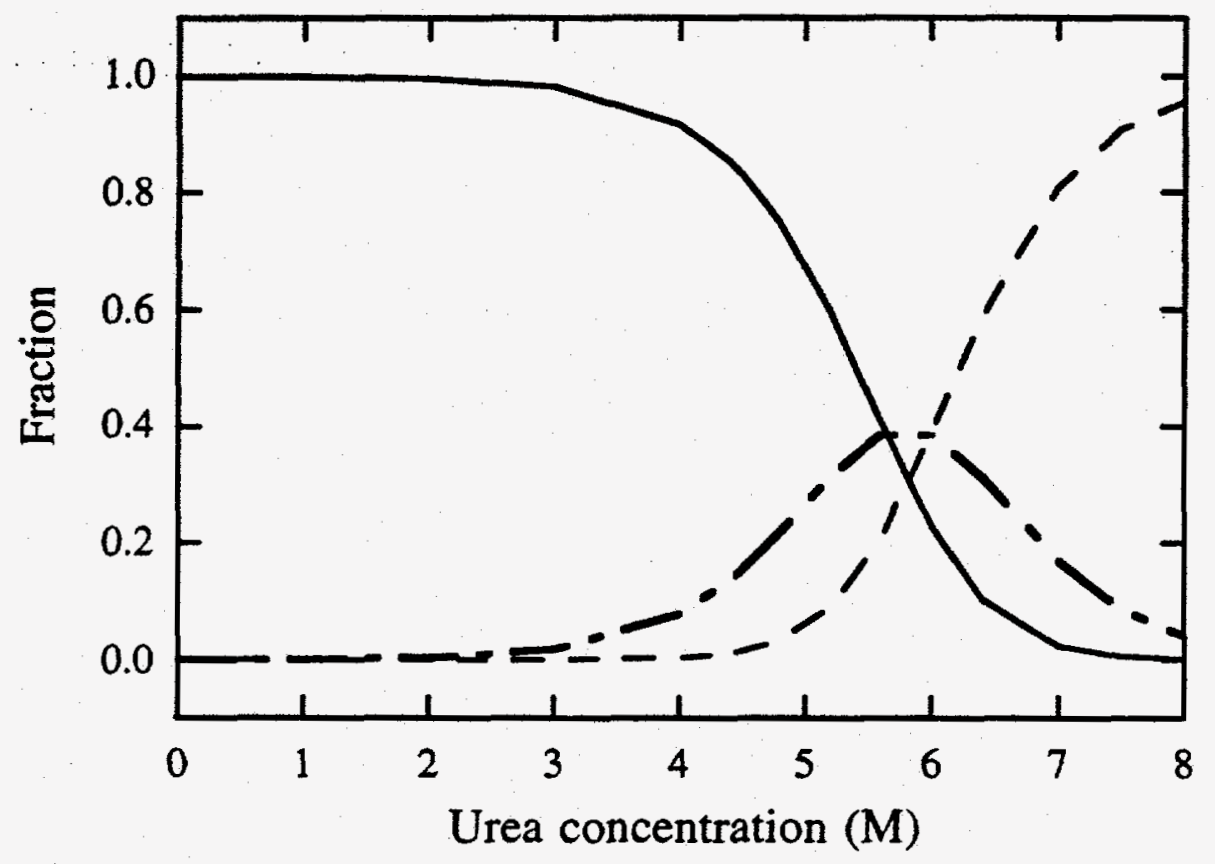

Figure 4.12. Population of native (-), intermediate (-- -) and unfolded states (- - ) of lysozyme at $\mathrm{pH} 2.9$ as a function of urea concentration as determined by $\mathrm{R}_{\mathrm{g}}$ analysis. 
out over raw data sets without smoothing and over data sets with 5-point smoothing. The results were the same as $\mathrm{j}$ the case of 3-point smoothing but with different noise levels.

The global reduced $\chi^{2}$ values with $L$ component approximation of Kratky data at pH 2.9 are 94, 16, and 1.14 for $\mathrm{L}=1,2,3$, respectively. A third component is also found necessary to get reduced $\chi^{2}$ to be around unity at $\mathrm{pH} 2.0$. A typical three-component approximation and its residuals are shown in Fig. 4.13. The three basis functions weighted by their singular values are presented in Fig. 4.14. Although basis functions are not scattering patterns of specific molecules, some correlations are apparent. For example, the first basis function resembles characteristics of a globular protein, and can be related to scattering of the native state. The second and third basis functions are complex, yet they have certain features in the high angle region that are similar to scattering of random coils or the unfolded state. The observation of three significant scattering basis functions suggests a minimum of three unique scattering species; that is, there is an intermediate in addition to the native and unfolded forms.

To estimate $b_{1}^{I}, b_{2}^{I}$ and $b_{3}^{I}$ and construct the scattering curve for the isolated intermediate, three columns of $V$ weighted by their singular values were simultaneously fit according to equation [17] with a set of thermodynamic parameters $\Delta G_{1}^{\mathrm{H}_{2} \mathrm{O}}, \Delta \mathrm{G}_{\mathrm{U}}^{\mathrm{H}_{2} \mathrm{O}}, \Delta n_{1}$ and $\Delta n_{U}$. The best fits were achieved where $\Delta G_{U}^{H_{2} O}$ and $\Delta n_{U}$ were in good agreement with those from denaturation curve analysis, although $\Delta \mathrm{G}_{1}^{\mathrm{H}_{2} \mathrm{O}}$ and $\Delta \mathrm{n}_{1}$ were only half of the values derived from denaturation curve analysis. It should be noted that plots of $b_{i}$ $(\mathrm{i}=1,2,3)$ versus urea concentrations (Fig. 4.15) are rather noisy, particularly the plot of $b_{3}$. On the other hand, both the $R_{g}$ and $C D$ denaturation curves (Fig. 4.11.a) are smooth, therefore parameters derived from them are considered to be accurate. Hence, data were fitted in such a way that the relevant thermodynamic parameters, $\Delta G_{1}^{\mathrm{H}_{2} \mathrm{O}}, \Delta \mathrm{G}_{\mathrm{U}}^{\mathrm{H}_{2} \mathrm{O}}, \Delta n_{\mathrm{I}}$ and $\Delta \mathrm{n}_{\mathrm{U}}$, were chosen to be close to those from the denaturation curve analysis. The fits for $b_{1}$ and $b_{2}$ were reasonable, whereas the result for $b_{3}$ was clearly less well defined. Again, the scattering data quality available at the present time may not be adequate for a reliable interpretation of the lysozyme unfolding mechanism taken on its own, but by considering it along with the analysis of the denaturation curves, the reliability of the fits is considerably increased.

A scattering curve for the intermediate was synthesized using the estimated $b_{1}^{I}, b_{2}^{I}$ and $b_{3}^{1}$ with equation [16]. The synthesized Kratky plot, shown in Fig. 4.16.a, appears to be a superposition of compact and random coil features (Doniach, et al., 1995), and 

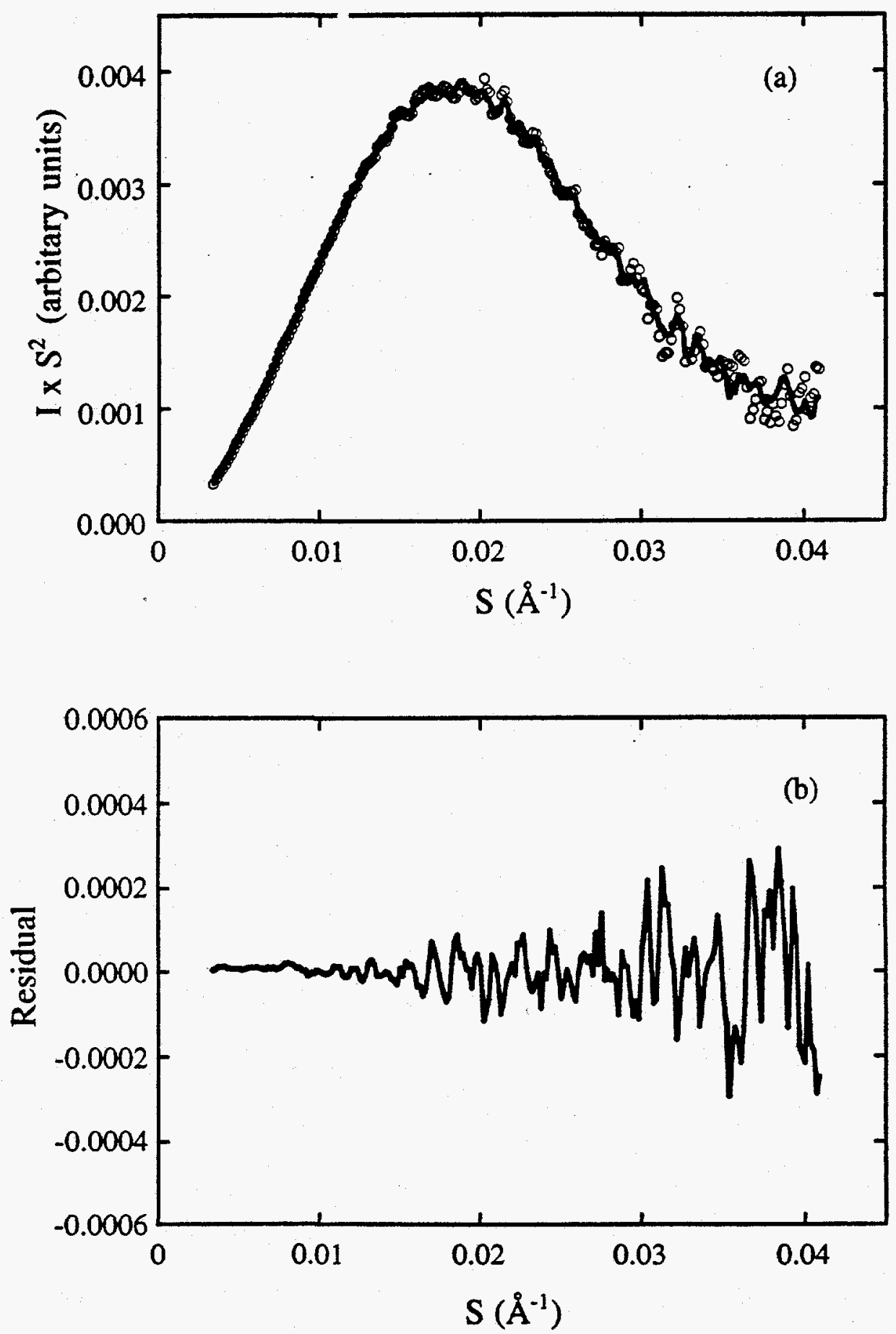

Figure 4.13. a) Representative Kratky plots of the data (o) and its three-component SVD approximation (-). The urea concentration is $4 \mathrm{M}$. b) The residuals, or differences between the experimental data and the calculation using three components. 

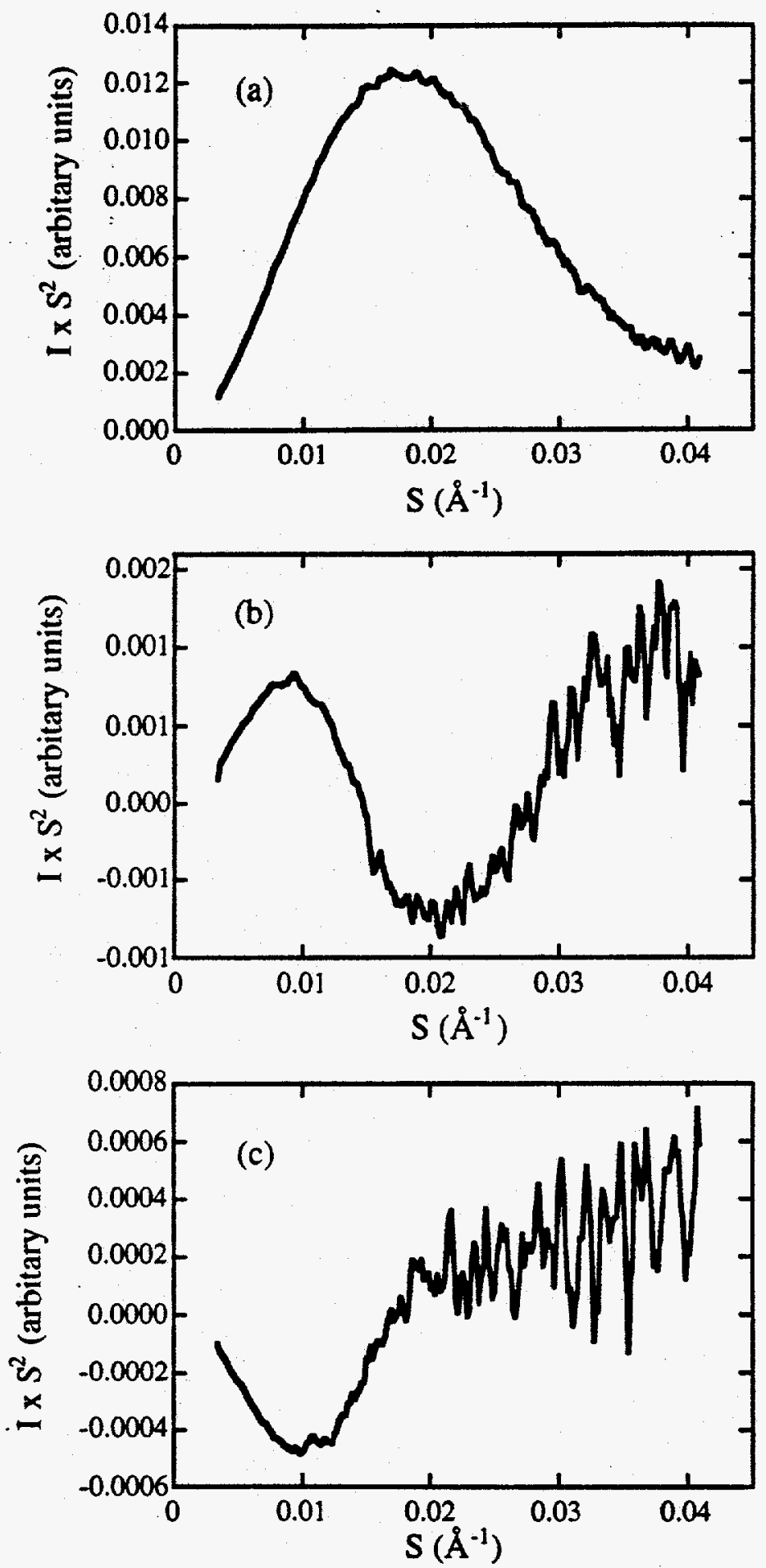

Figure 4.14. The orthogonal basis scattering functions (columns of matrix $U$ ) in Kratky form from SVD analysis. The curves are weighted by their singular values: a) the first function $\mathrm{U} 1$ by 0.118 ; b) the second function $\mathrm{U} 2$ by 0.004 ; ) the third function $\mathrm{U} 3$ by 0.002 . 

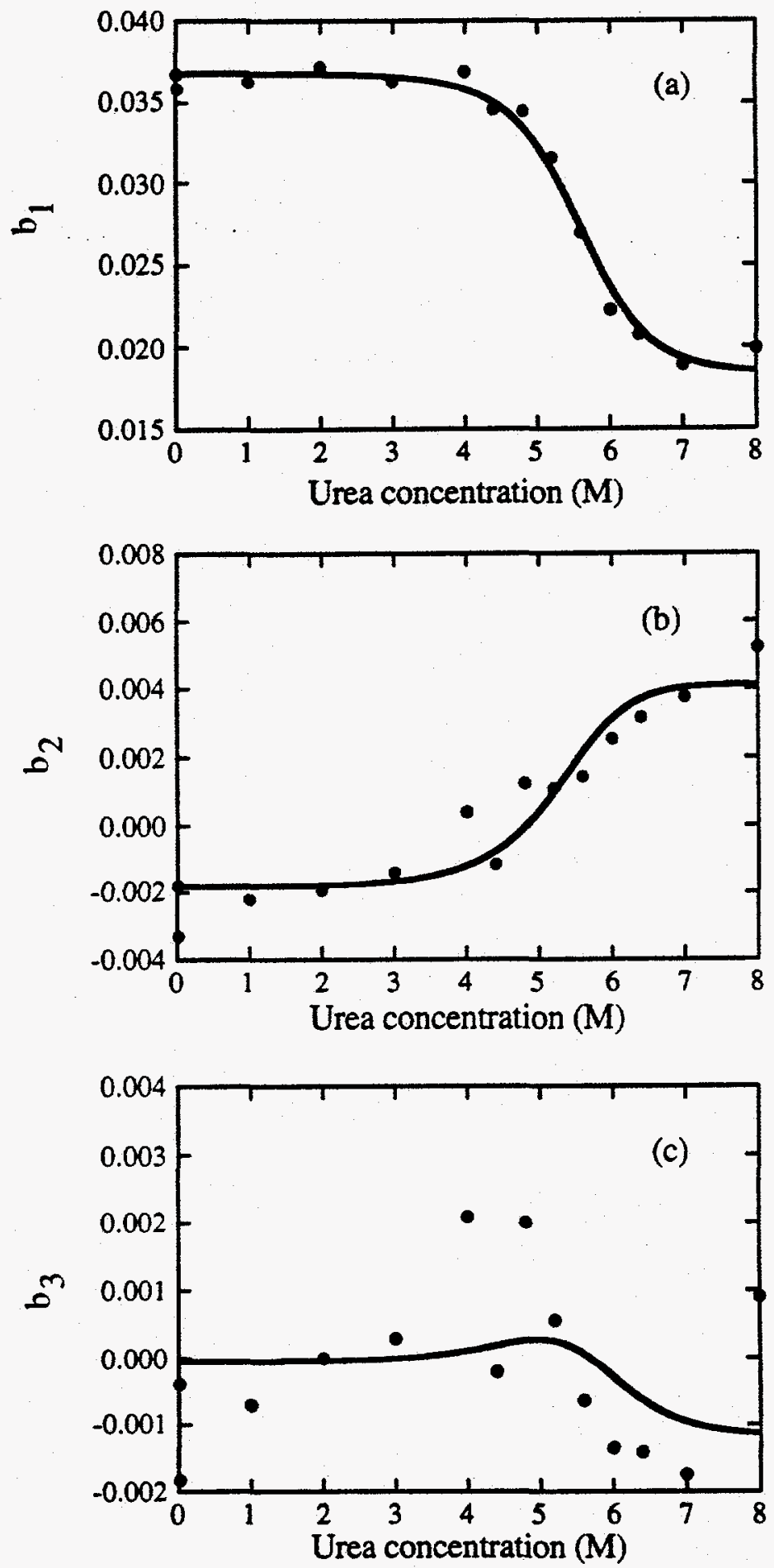

Figure 4.15. Singular value-weighted urea dependence of basis functions a) $\mathbf{U} 1$; b) $\mathbf{U} 2$; c) U3. The solid lines are results of simultaneous nonlinear least-squares fits with equation [17]. 

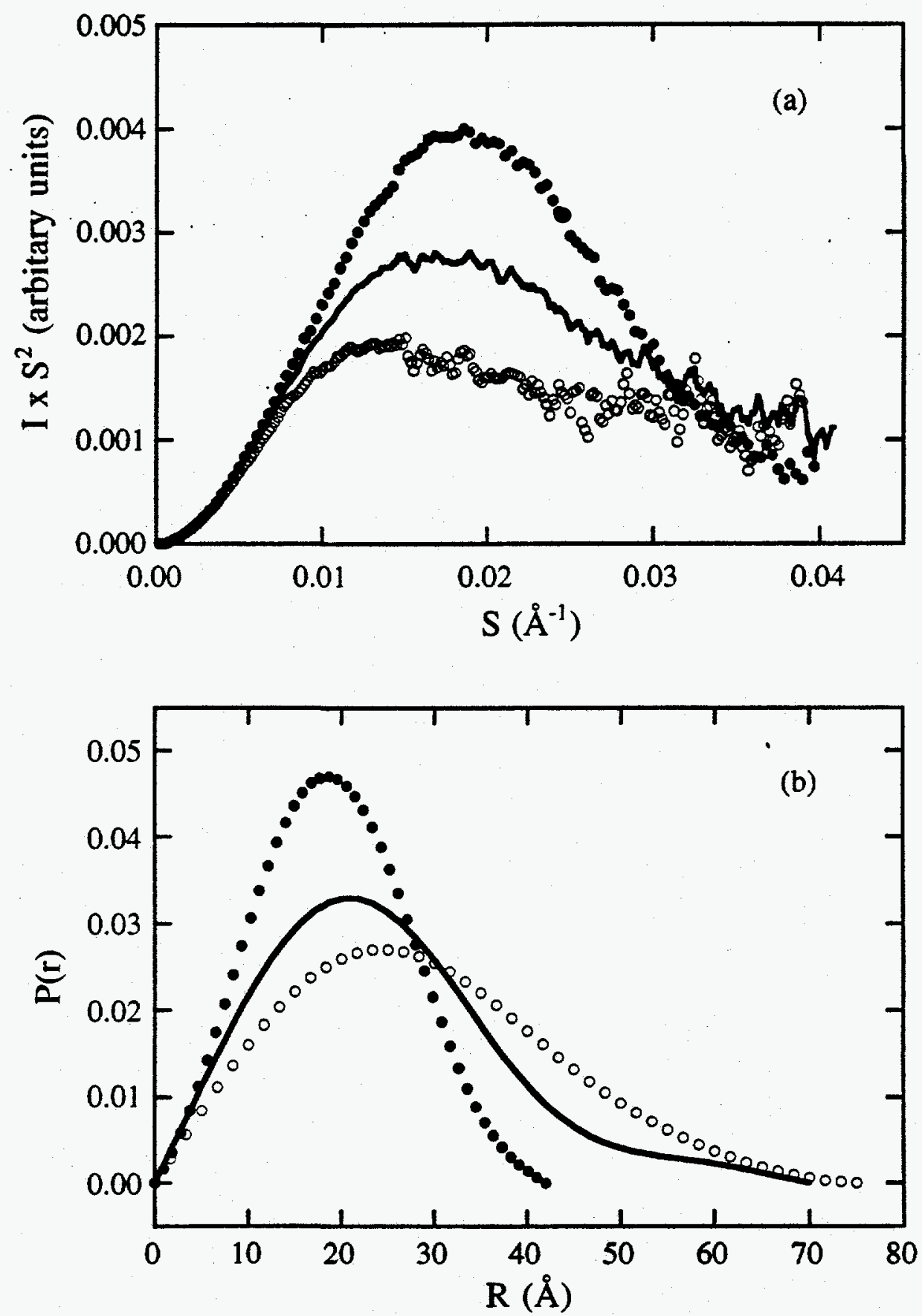

Figure 4.16. (a) The Kratky plot of a three-component SVD construction of the intermediate state $(-)$. (b) The $P(r)$ function for the synthesized intermediate state shown in (a). Data from both the native state $(\bullet)$, and the unfolded state in $8 \mathrm{M}$ urea $\mathrm{pH}$ 2.9 (o) are also included for comparison. Data shown in the Kratky plots were after with 3-point smoothing. 
suggests that the globularity of the intermediate is closer to that of the native rather than to the unfolded state in $8 \mathrm{M}$ urea. Fig. 4.16.b shows the $\mathrm{P}(\mathrm{r})$ function calculated from this constructed scattering curve. The peak position, $-20 \AA$, is close to that of the native, while the maximum distance of the intermediate, $D_{\max }$, has extended to that of the unfolded state $(\sim 70 \AA)$. The features in the $\mathrm{P}(\mathrm{r})$ function of the intermediate indicate again that the conformation of the intermediate is midway between those of the native and the unfolded states. The $\mathrm{R}_{\mathrm{g}}$ for this intermediate is $-19 \AA$ from the Guinier approximation, in good agreement with $\sim 20 \AA$ from the $R_{\mathrm{g}}$ denaturation curve analysis.

Structural models of the intermediate state. A kinetic intermediate has been identified in the refolding pathway of lysozyme which consists of a compact $\alpha$ domain showing H-D exchange protection factors of a molten globule character and a highly disordered $\beta$ domain (Radford, et al., 1992).

It is of interest to try and estimate whether our result of $R_{g} \sim 19-20 \AA$ is compatible with this structural model. To do this one can use the fact that the observed $R_{g}$ may be decomposed into contributions from disjoint fragments of the protein. For simplicity each atom (not including hydrogens) in the protein is assumed to have the same scattering factor (this could easily be generalized to include more accurate estimates). One can write

$$
\mathrm{R}_{\mathrm{g}}^{2}=\frac{1}{\mathrm{n}} \sum_{\mathrm{i}}\left(\overrightarrow{\mathrm{r}}_{\mathrm{i}}-\overrightarrow{\mathrm{r}}_{\mathrm{cm}}\right)^{2}
$$

where $n$ is the total number of atoms, with positions $\vec{r}_{i}$, and $\vec{r}_{c m}$ is the center of mass coordinate vector of the protein. Then for any disjoint subsets $A$ and $B$, with $n_{a}$ and $n_{b}$ atoms

$$
\begin{aligned}
R_{g}^{2} & =\frac{1}{n}\left\{\sum_{A}\left(\vec{r}_{i}-\vec{r}_{c A}\right)^{2}+n_{A}\left(r_{c A}-r_{c m}\right)^{2}+\sum_{B}\left(\vec{r}_{i}-\vec{r}_{c B}\right)^{2}+n_{B}\left(r_{c B}-r_{c m}\right)^{2}\right\} \\
& =\frac{n_{A}}{n}\left\{R_{g, A}^{2}+\left(r_{c A}-r_{c m}\right)^{2}\right\}+\frac{n_{B}}{n}\left\{R_{g, B}^{2}+\left(r_{c B}-r_{c m}\right)^{2}\right\}
\end{aligned}
$$

where $\vec{r}_{\mathrm{cA}}$ is the center of mass of subset $A$ and similarly for $B$. (This generalizes to an arbitrary number of disjoint subsets.) Using the atomic coordinates for native lysozyme these quantities have been evaluated for 3 subsets: $\alpha 1$ (residues 1-35), $\beta$ (35-76) and $\alpha 2$ (76-129). The sets were divided at the $\alpha$ carbons. The resulting values are given in Table 4.2. In this approximation (for the scattering factors) the total $\mathrm{R}_{\mathrm{g}}$ comes out to $13.9 \AA$, 
somewhat smaller than $15.3 \AA$ from solution scattering. A smaller computed $R_{g}$ is expected because, in addition to the approximate nature of the calculation, the measured $R_{g}$ from solution scattering includes the effects of the hydration layers formed around the lysozyme molecules in solution.

In order to estimate $\mathrm{R}_{\mathrm{g}}$ for the intermediate partially folded state in the refolding pathway (Radford, et al., 1992), one needs a model for an unfolded conformation of the $\beta$ region. To do this realistically is a complex task which has not yet been done for a protein domain in contact with the rest of the protein in its native conformation. Therefore selected highly simplified models of the $\beta$ domain were examined in order to get an estimate of the range of possible values of $R_{g, \beta}$ and $\left|\vec{r}_{c, \beta}-\vec{r}_{c m}\right|$ for the unfolded domain. To do this, the backbone was broken up into 10 segments of about 4 residues each. The idea here was that these fragments represent roughly the persistence length appropriate for disordering the polymer chain (Damaschun, et al., 1991). The sum of the length of the fragments is found to be $104 \AA$. An extreme overestimate to the radius of gyration is obtained by taking an extended loop from residue $\left(C_{\alpha}\right)_{35}$ to $\left(C_{\alpha}\right)_{76}$. Taking this in the form of a circle oriented away from the protein center of mass it was found that $\left(R_{g, \beta}^{\text {circle }}\right)^{2} \cong 324 \AA^{2}$ and $\left(\overline{\mathrm{r}}_{\mathrm{c}, \beta}^{\text {circle }}-\overline{\mathrm{r}}_{\mathrm{cm}}\right)^{2} \cong 533 \AA^{2}$. This gives an upper boundary on $R_{\mathrm{g}}$ for the whole protein of $\mathrm{R}_{\mathrm{g}}^{\text {total }}<20.4 \AA$, which may correspond to $21-22 \AA$ when considering the hydration factor. A second, somewhat more compact but still extended, model was taken to consist of a linear arrangement of four fragments (each $10.4 \AA$ ) oriented away from the center of mass then returning with an extra fold in the form of a zig-zag. This conformation gives $\left(R_{\mathrm{g}, \beta}^{\text {linear }}\right)^{2} \equiv 159 \AA$ and $\left(\vec{r}_{\mathrm{c}, \beta}^{\text {circle }}-\overrightarrow{\mathrm{r}}_{\mathrm{cm}}\right)^{2} \equiv 460 \AA$. The resulting $\mathrm{R}_{\mathrm{g}}$ estimate is $\mathrm{R}_{\mathrm{g}}^{\text {total }}$ $=18.3 \AA$, which again may increase to $19-20 \AA$ when a hydration layer is taken into account. Since even the second model is probably more extended than would be expected for a realistic random coil conformation of the $\beta$ subset, it is seen that the observed value for $R_{g}$ of the intermediate, $\sim 19-20 \AA$, falls close to the upper end of the range of possible values for a model in which the $\alpha 1, \alpha 2$ subunits retain their native values. It is therefore concluded that the $\alpha 1$ and $\alpha 2$ components of the protein are likely to be somewhat disordered in the urea-induced intermediate state even though the $\mathrm{CD}$ evidence suggests that the $\alpha$ helical component of the intermediate is very close to that of the native. This may imply some disordering of the turns linking the $\alpha$ helical structures with some accompanying swelling of the $\alpha 1, \alpha 2$ components. 
Table 4.2. Fragments of lysozyme, and their radii of gyration.

\begin{tabular}{llll}
\hline Subset & $\alpha 1(1-35)^{\mathrm{a}}$ & $\beta(35-76)^{\mathrm{a}}$ & $\alpha 2(76-129)^{\mathrm{a}}$ \\
\hline number of atoms & 270 & 356 & 368 \\
subset $\mathrm{R}_{\mathrm{g}}(\AA)^{\mathrm{b}}$ & 9.8 & 10.4 & 13.2 \\
$\left|\overrightarrow{\mathrm{r}}_{\mathrm{c}, \text { subset }}-\overrightarrow{\mathrm{r}}_{\mathrm{cm}}\right|^{2}\left(\AA^{2}\right)^{\mathrm{b}}$ & 69.0 & 109.0 & 19.1 \\
\hline
\end{tabular}

$a$ residue number.

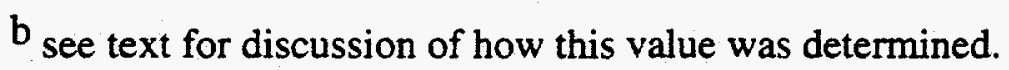




\subsection{Discussion}

Our results from the $\mathrm{x}$-ray solution scattering study provide evidence for a thermodynamically stable intermediate in the equilibrium unfolding of hen egg-white lysozyme by urea at $\mathrm{pH}$ 2.9. Three significant basis functions from the SVD analysis of the scattering curves (Kratky plots) suggest a minimum of three distinct scattering components in the urea-induced unfolding of hen lysozyme at $\mathrm{pH}$ 2.9. A third species is also implicated from the lack of coincidence of the denaturation curves measured by $R_{g}$ and the CD signals at $222 \mathrm{~nm}$; the transition in $\mathrm{CD}$ appears to be highly cooperative, whereas the cooperativity revealed by $R_{\mathrm{g}}$ tends to be less pronounced. The intermediate is assumed to have a similar CD signal at $222 \mathrm{~nm}$ to that of the native state. The radius of gyration of the intermediate is found to be around 19-20 $\AA$, and a scattering curve for this intermediate is constructed.

Structures of native and unfolded states. The stability of hen egg-white lysozyme is remarkable. No significant conformational change has been observed over $\mathrm{pH} 1.2-11.3$ in the absence of denaturant (Imoto, et al., 1972). The radius of gyration of lysozyme measured at pH 2.9, 15.3 $\AA$, falls within the reported values of 14.3 to $16.0 \AA$ (Imoto, $e t$ al., 1972). The $\mathrm{P}(\mathrm{r})$ function of lysozyme in $\mathrm{pH} 2.9$ solution also agrees well with that computed from the crystal structure, so that within the resolution of the $\mathrm{x}$-ray scattering data, the conformations of lysozyme in solution of $\mathrm{pH} 2.9$ and in the crystalline form are essentially the same. Similar results are derived at $\mathrm{pH} 2.0$. It is concluded therefore that lysozyme molecules exist in the native state under the conditions studied.

Thermodynamic standard functions of lysozyme over temperatures $0-100^{\circ} \mathrm{C}$ and pH 2-7 have been reported (Pfeil \& Privalov, 1976), and the conformational stability of lysozyme $\left(\Delta \mathrm{G}_{\mathrm{U}}^{\mathrm{H}_{2} \mathrm{O}}\right)$ can be calculated accordingly. At $\mathrm{pH} 3.0$ and $20^{\circ} \mathrm{C}, \Delta \mathrm{G}_{\mathrm{U}}^{\mathrm{H}_{2} \mathrm{O}}$ is $\sim 12$ $\mathrm{kcal} / \mathrm{mol}$ by calorimetric measurements. Estimates of $\Delta \mathrm{G}_{\mathrm{U}}^{\mathrm{H}_{2} \mathrm{O}}$ from denaturation curve analysis, however, range from $6 \mathrm{kcal} / \mathrm{mol}$ to $14 \mathrm{kcal} / \mathrm{mol}$ in $\mathrm{pH} 3.0$ to 6 at $25^{\circ} \mathrm{C}$, depending on the method employed to extrapolate $\Delta \mathrm{G}_{\mathrm{U}}$ to zero denaturant concentration. Nevertheless, these values are reasonable for an estimate of $10-20 \mathrm{kcal} / \mathrm{mol}$ for small globular proteins. By using the denaturant binding model (which normally gives a higher estimate for $\Delta \mathrm{G}_{\mathrm{U}}^{\mathrm{H}_{2} \mathrm{O}}$ but which provides a better picture of the denaturant effect among other methods (Pace, 1986)), values of $11.6( \pm 0.5) \mathrm{kcal} / \mathrm{mol}$ and $8( \pm 0.2) \mathrm{mol} / \mathrm{kcal}$ are derived from $2 \& 3$-state analysis and $2 \& 2$-state analysis, respectively. A difference in 
$\Delta \mathrm{G}_{\mathrm{U}}^{\mathrm{H}_{2} \mathrm{O}}$ is reported for the $\alpha$ subunit of trytophan synthase with $-12 \mathrm{kcal} / \mathrm{mol}$ from 3-state analysis and $\sim 3.6 \mathrm{kcal} / \mathrm{mol}$ from 2-state analysis (Matthews \& Crisanti, 1981). Although both analysis models yield a good estimate of $\Delta \mathrm{G}_{\mathrm{U}}^{\mathrm{H}_{2} \mathrm{O}}$ for lysozyme, SVD of the scattering curves supports the existence of a distinct scattering intermediate.

In the denaturant binding model, the protein unfolds as a result of an increase in urea binding sites in the unfolded state compared with the native state. In $8 \mathrm{M}$ urea solution, a value of $77 \mathrm{~mol}$ urea per mol lysozyme is calculated by assuming two peptide bonds interacting with one urea molecule and one aromatic residue including histidine associating with one urea molecule (Prakash, et al., 1981), while $65 \mathrm{~mol} / \mathrm{mol}$ is derived from partial specific volume measurements (Prakash, et al., 1981). $\Delta \mathrm{n}_{U}$, the difference in the number of urea binding site between the unfolded state and the native state, is expected to be smaller because some of these binding sites are also accessible to urea in the native conformation. Both values of $49 \mathrm{~mol} / \mathrm{mol}$ from the $2 \& 3$-state analysis and $33 \mathrm{~mol} / \mathrm{mol}$ from the 2\&2-state analysis appear to be plausible, but again the SVD analysis of the scattering data justifies the existence of an intermediate.

The rather small $\mathrm{R}_{\mathrm{g}}, \sim 22 \AA$, of lysozyme in $8 \mathrm{M}$ urea, when compared with $32 \AA$ of a random coil model of similar molecular weight, heparin, suggests that the denatured lysozyme in $8 \mathrm{M}$ urea pH 2.9 does not resemble a random coil. A slightly larger $R_{\mathrm{g}}, \sim 24$ $\AA$, is obtained for reduced unfolded lysozyme in $8 \mathrm{M}$ urea with $80 \mathrm{mM}$ DTT. The shape of the $P(r)$ function of the denatured state as well as its markedly small maximum dimension, $D_{\text {max }}$, further support a departure from a random coil structure. Furthermore, the Kratky plot provides a powerful way to analyze the scattering data in distinguishing between coil and globular structures (Glatter \& Kratky, 1981). Although the lack of a well-defined peak in the Kratky plot demonstrates a significant reduction in globularity in unfolded lysozyme, the absence of a plateau and the presence of a residual peak shape argue against a coil structure. The non-globularity of the disulfide-reduced unfolded lysozyme appears to increase further as judged from the Kratky plot (data not shown), but still deviates from that of a coil.

The degree of unfolding and the structure of the unfolded state are among the interesting issues in protein folding studies. Although far-UV CD and amide hydrogen exchange experiments suggest that lysozyme in $8 \mathrm{M}$ urea pH 2.0 is largely unstructured (Buck, et al., 1994), evidence for nonspecific interactions and deviation from random coil is shown from ${ }^{1} \mathrm{H}$ NMR as well as photo-CIDNP spectra (Broadhurst, et al., 1991; Evans, 
et al., 1991). Our scattering results clearly support a non-random coil structure in the denatured state of lysozyme in $8 \mathrm{M}$ urea at $\mathrm{pH} 2.9$. The residual structure appears to be only slightly affected by removal of disulfide bridges, arguing for existence of nonspecific interactions in addition to the geometric constraints imposed by covalent S-S bonds.

Evidence for an intermediate state. Cooperative unfolding does not necessary indicate a two-state transition with absence of equilibrium intermediates. The calculated three-state transition curves also appear to be quite cooperative, and there seems to be no clear boundary between two-state and three-state transitions (Lumry, et al., 1966). A practical approach is to use different structural probes to follow the unfolding process under the same conditions, and the existence of stable intermediates may be inferred from the differences in the unfolding curves. In our study, a small yet visible difference in the unfolding curves of lysozyme in urea solution $\mathrm{pH} 2.9$ is observed by $\mathrm{CD} 222 \mathrm{~nm}$ and $\mathrm{R}_{\mathrm{g}}$. This difference may reflect the occurrence of an intermediate as it usually does, or since the distinction is subtle, it might nevertheless be a result of poor accuracy and precision of the techniques as well as any variations associated with the sample (in our case this might result from the difference of lysozyme concentration $\sim 0.6 \mathrm{mg} / \mathrm{ml}$ in $\mathrm{CD}$ versus $\sim 7 \mathrm{mg} / \mathrm{ml}$ in scattering experiments). However, a strong argument for the existence of an intermediate comes from the SVD analysis of scattering data over the whole scattering angle region with the evidence of a third basis function. Our results stress the importance of applying various techniques to probe different aspects of protein structure.

An alternate to a three-state binding model for denaturation-induced unfolding has been put foward by Alonso and Dill (Alonso \& Dill, 1991; Dill \& Shortle, 1991). Their picture, which might be thought of as a "many-state" model is that there is a single free energy barrier for going from the native to an ensemble of disordered states which comprise structures varying from the compact conformation with a hydrophobic core to the fully unfolded conformation; however, the degree of unfolding of the disordered states is a continuous function of denaturant concentration. (See also the discussion in Doniach, et al., 1995.) Although this provides a rather physically plausible scenario, the SVD analysis of our data shows that the signal/noise level attained in our measurements is probably not good enough to discriminate this continuous unfolding picture from a 3-state picture. In particular, Fig. 4.12 shows that at urea concentration between $6.5 \mathrm{M}$ and $8 \mathrm{M}$ the scattering profiles may be expressed as a linear superposition of the scattering profile from the partially folded intermediate and from the fully denatured state. If there is a gradual unfolding of the $\alpha$ domain over this concentration range, it would show up as additional 
basis vectors in the SVD matrix. However, it was found that components of the SVD matix with smaller singular values than the first three lie within the noise limits of the data, so they could not be discriminated for at the present signal/noise levels.

Structure of the intermediate. By assumption of a two-state approximation to $\mathrm{CD}$ measurements and the observation of unfolding at lower urea concentration detected by $R_{g}$ than by $C D$, the intermediate has a similar $C D$ signal at $222 \mathrm{~nm}$ to that of the native state. The compactness of this intermediate is characterized by $19-20 \AA$ in $R_{g}$. Compared with the native $(\sim 15 \AA)$ and the unfolded $(-22 \AA)$ states, the structure of the intermediate lies halfway between the native and the unfolded conformations, suggesting a great decrease in tertiary contacts in this intermediate. The constructed $\mathrm{P}(\mathrm{r})$ function for the intermediate shows that while the intermediate is swollen to such an extend that its maximum length is close to that in the unfolded state, the most populated intramolecular distances, measured by the peak position, remain similar to that in the native state. The intermediate must maintain some similar local structure present in the native conformation. A well-defined peak in the calculated Kratky plot indicates that the shape of the intermediate is still globular. Structural modeling suggests that the substantially enlarged $\mathbf{R}_{\mathbf{g}}$ for the intermediate may not be solely attributed to a fully unfolded $\beta$ domain; instead, it appears that the $\alpha$ domain ( $\alpha 1, \alpha 2$ subsets) also expands to some extent. Taken together, our results suggest that the intermediate has native-like secondary structure for the $\alpha$ domain with a distorted compact fold, characteristic of a molten globule-like state, and an unfolded structure for the $\beta$ domain.

The intermediary conformation of the partially folded state is also supported by the derived thermodynamic parameters: $\Delta \mathrm{G}_{1}^{\mathrm{H}_{2} \mathrm{O}}$ is about half of $\Delta \mathrm{G}_{\mathrm{U}}^{\mathrm{H}_{2} \mathrm{O}}$. Partially folded states with intermediate stability are also found in urea-unfolding of trytophan synthase and B-lactamase (Matthews \& Crisanti, 1981; Uversky \& Ptitsyn, 1994). From the denaturant binding model, only half the urea binding sites in the unfolded state of lysozyme are available to urea in the transformation of $\mathrm{N} \rightarrow \mathrm{I}$; the other half becomes exposed in the unfolding of I $>\mathrm{U}$. Our results for lysozyme unfolding in urea solution $\mathrm{pH} 2.9$ can be interpreted in the framework of the hierarchical unfolding model (Palleros, et al., 1993). In this model, the native conformation consists of structural units. The initial unfolding observed by $R_{g}$ would be correlated with a breakdown of interunit interactions between the $\alpha$ domain and the $\beta$ domain, and unfolding of the $\beta$ domain. The further unfolding detected by the CD signal at $222 \mathrm{~nm}$ would be associated with the disruption of the $\alpha$ domain (including secondary interactions as well as the side-chain interactions within the 
unit). The structure of the intermediate would be expected to maintain compactness (19-20 $\AA)$ to some extest since it retains some weak interactions besides containing substantial amount of secondary structure, particularly in the $\alpha$ domain.

The importance of our study is two-fold. First, although stable partially folded states for hen lysozyme are scarce, here we demonstrate that under appropriate conditions, intermediates can be accumulated at a level sufficient for characterization. Second, the partially compact, native-like secondary structure in the intermediate exhibits certain features of the early kinetic counterpart (Radford, et al., 1992), and structural characterization of this intermediate may provide a model to help in elucidating the early and fast events in the protein folding process. Our recent time-resolved scattering studies show that the radius of gyration of the first resolved conformation ( $-100 \mathrm{~ms}$ after initiation of refolding in urea at $\mathrm{pH} 2.9$ ) is $\sim 19 \AA$, a value close to that of the intermediate (see Chapter 5).

\subsection{Acknowledgments}

I would like to thank Daniel Segel for design of the flow cell, and Dr. Hirotsugu Tsuruta and Daniel Segel for the assistance in data acquisition. We wish to acknowledge Prof. Robert L. Baldwin of Biochemistry Department, Stanford University for the use of the CD spectropolarimeter. I would like to thank Dr. David G. Lambright for the SVD program, and Xiaohua Huang for computing assistance in this study. This research was supported by National Institutes of Health Grant RR-01209 (to K.O.H.). Data were collected at beam line 4-2 at SSRL which is supported by the U.S. Department of Energy, Office of Basic Energy Sciences, and in part by the National Institutes of Health, Biomedical Research Technology Program, National Center for Research Resources and by the Department of Energy, Office of Health and Environmental Research.

\subsection{References}

Alonso, D. O. V., \& Dill, K. A. (1991). Biochemistry, 30, 5974-5985.

Anfinsen, C. B. (1973). Science, 181, 223-230.

Aune, K. C., Salahuddin, A., Zarlenyo, M. H., \& Tanford, C. (1967). J. Biol. Chem., 242, 4486-4489. 
Aune, K. C., \& Tanford, C. (1969). Biochemistry, 8(11), 4586-4590.

Baum, J., Dobson, C. M., Evans, P. A., \& Hanley, C. (1989). Biochemistry, 28, 7-13.

Blake, C. C., Koening, D. F., Mair, G. A., North, A. C. T., Phillips, D. C., \& Sarma, V. R. (1965). Nature, 206, 757-761.

Blundell, T. L., \& Johnson, M. S. (1993). Prot. Sci., 2, 877-883.

Bradbury, J. H., \& King, N. L. R. (1969). Nature, 223, 1154-1156.

Broadhurst, R. W., Dobson, C. M., Hore, P. J., Radford, S. E., \& Rees, M. L. (1991). Biochemistry, 30, 405-412.

Bruzzesi, M. R., Chiancone, E., \& Antonini, E. (1965). Biochemistry, 9, 1796-1800.

Buck, M., Radford, S. E., \& Dobson, C. M. (1993). Biochemistry, 32, 669-678.

Buck, M., Radford, S. E., \& Dobson, C. M. (1994). J. Mol. Biol., 237, 247-254.

Dael, H. V., Haezebrouck, P., Morozova, L., Arico-Muendel, C., \& Dobson, C. M. (1993). Biochemistry, 32, 11886-11894.

Damaschun, G., Damaschun, H., Gast, K., Gernat, C., \& Eirwer, D. (1991). Biochim. Biophys. Acta, 1078(2), 289-295.

Dill, K. A., Bromberg, S., Yue, K., Fiebig, K. M., Yee, D. P., Thomas, P. D., \& Chan, H. S. (1995). Protein Science, 4, 561-602.

Dill, K. A., \& Shortle, D. (1991). Annu. Rev. Biochem., 60, 795-825.

Dobson, C. M. (1994). Curr. Biol., 4(7), 636-640.

Dobson, C. M., Evans, P. A., \& Radford, S. E. (1994). Tibs, 19, 31-37.

Doniach, S., Bascle, J., Garel, T., \& Orland, H. (1995). J. Mol. Biol., 254(5), 960-967.

Eliezer, D., Chiba, K., Tsuruta, H., Doniach, S., Hodgson, K. O., \& Kihara, H. (1993). Biophys. J., 65, 912-917.

Evans, P. A., Topping, K. D., Woolfson, D. N., \& Dobson, C. M. (1991). Proteins: Struct. Funct. Genet., 9, 248-266.

Fetler, L., Tauc, P., Herve, G., Moody, M. F., \& Vachette, P. (1995). J. Mol. Biol., 251, 243-255.

Glatter, O., \& Kratky, K. (Ed.). (1981). Small-Angle X-Ray Scattering. New York: Academic Press.

Golub, G. H., \& Reinsch, C. (1970). Numer. Math, 14, 403-420.

Greene, R. F., \& Pace, C. N. (1974). J. Biol. Chem., 249, 5388-5393.

Haezebrouck, P., Joniau, M., Dael, H. V., Hooke, S. D., Woodruff, N. D., \& Dobson, C. M. (1995). J. Mol. Biol., 246(3), 382-387.

Hofrichter, J., Sommer, J. H., Henry, E. R., \& Eaton, W. A. (1983). Proc. Natl. Acad. Sci. USA, 80, 2235-2239. 
Imoto, T., Johnson, L. N., North, A. C. T., Phillips, D. C., \& Rupley, J. A. (1972). Vertebrate Lysozyme. In Boyer, P. D. (Eds.), The Enzymes (pp. 666-868). New York: Academic Press.

Jennings, P. A., \& Wright, P. E. (1993). Science, 262, 892-896.

Karplus, M., \& Weaver, D. L. (1976). Nature, 260, 404-406.

Kataoka, M., Nishii, I., Fujisawa, T., Ueki, T., Tokunaga, F., \& Goto, Y. (1995). J. Mol. Biol., 249(1), 215-228.

Kato, S., Okamura, M., Shimamoto, N., \& Utiyama, H. (1981). Biochemistry, 20, 1080-1085.

Kim, P. S., \& Baldwin, R. L. (1990). Annu. Rev. Biochem., 59, 631-660.

Kuwajima, K., Nitta, K., Yoneyama, M., \& Sugai, S. (1976). J. Mol. Biol., 106, 359373.

Lambright, D. G., Balasubramanian, S., \& Boxer, S. G. (1991). Chem. Phys., 158, 249-260.

Lattman, E. E. (1994). Curr. Opin. Struct. Biol., 4, 87-92.

Liberti, P. A., \& Stivala, S. S. (1967). Arch. Biochem. Biophys., 119, 510-518.

Lumry, R., Biltonen, R., \& Brandts, J. F. (1966). Biopolymers, 4, 917-944.

Matthews, C. R., \& Crisanti, M. M. (1981). Biochemistry, 20, 784-792.

Ohgushi, M., \& Wada, A. (1983). FEBS, 164, 21-24.

Pace, C. N. (1975). Crit. Rev. Biochem., 3, 1-43.

Pace, C. N. (1986). Determination and analysis of urea and guanidine hydrochloride denaturation curves. In Colowick, S. P. \& Kaplan, N. O. (Eds.), Methods in Enzymology (pp. 266-280). Orlando, Florida: Academic Press.

Palleros, D. R., Shi, L., Reid, K. L., \& Fink, A. L. (1993). Biochemistry, 32, 4314 4321.

Pfeil, W., \& Privalov, P. L. (1976). Biophys. Chem., 4, 41-50.

Prakash, V., Loucheux, C., Scheufele, S., Gorbunoff, M. J., \& Timasheff, S. N. (1981). Arch. Biochem. Biophys., 210(2), 455-464.

Press, W. H., Teukolsky, S. A., Vetterling, W. T., \& Flannery, B. P. (1992). Numerical Recipes in Fortran (2nd ed.). Cambridge University Press.

Provencher, S. W., \& Blockner, J. (1983). J. Biochem. Biophys. Methods, 7, 331-334.

Ptisyn, O. B. (19.73). Dokl. Acad. Nauk SSSR, 210, 1213-1215.

Ptisyn, O. B. (1993). The molten globule state. In Creighton, T. E. (Eds.), Protein folding (pp. 243-300). New York: W. H. Freeman and Company. 
Ptitsyn, O. B. (1995a). Molten Globule and Protein Folding. In Anfinsen, C. B., Edsall, J. T., Richards, F. M., \& Eisenberg, D. S. (Eds.), Advances in Protein Chemistry (pp. 83-230). San Diego: Academic Press, Inc.

Ptitsyn, O. B. (1995b). Curr. Opin. Struct. Biol., 5, 74-78.

Radford, S. E., Dobson, C. M., \& Evans, P. A. (1992). Nature, 358, 302-307.

Schmid, F. X. (1993). Kinetics of unfolding and refolding of single-domain proteins. In Creighton, T. E. (Eds.), Protein folding (pp. 197-242). New York: W. H. Freeman and Company.

Semenyuk, A. V., \& Svergun, D. I. (1991). J. Appl. Crystallogr., 24, 537-540.

Shrager, R. I., \& Hendler, R. W. (1982). Anal. Chem., 54, 1147-1152.

Stephenson, G. B. (1988). Nucl. Instrum. Meth. Phys. Res., A266(1-3), 447-451.

Svergun, D. I., Barberato, C., \& Koch, M. H. (1995). J. Appl. Crystallogr, submitted for publication.

Tanford, C. (1970). Protein denaturation. Part C. Theoretical models of the mechanism of denaturation. In C. B. Anfinsen, J., Edsall, J. T., \& Richards, F. M. (Eds.), Adv. Prot. Chem. (pp. 1-95). New York: Academic Press.

Uversky, V. N., \& Ptitsyn, O. B. (1994). Biochemistry, 33, 2782-2791.

Wetlaufer, D. B. (1962). Ultraviolet spectra of proteins and amino acids. In Advances in protein chemistry (pp. 303-390). New York: Academic Press, Inc. 


\section{Chapter 5}

Time-resolved Small-angle X-ray Scattering Studies of Lysozyme Refolding 


\subsection{Introduction and Background}

The complexity of kinetic study of protein folding arises from two major sources (Schmid, 1992). (1) The unfolded state of proteins is conformationally heterogeneous, which leads to multiple parallel refolding pathways. (2) Small globular proteins usually refold within seconds, and folding intermediates only form transiently in the refolding pathways.

To study the mechanism of folding reaction, two working models have been generalized. The framework model (Ptitsyn, 1973) suggests that a stable framework of secondary structure is formed before the native-like tertiary fold is formed. The modular assembly model (Karplus \& Weaver, 1976; Kim \& Baldwin, 1990) postulates that a protein folds by parts: the secondary and tertiary structures are formed at the same time within each folding units, or microdomains, transiently and independently, and these microdomains diffuse, collide and coalesce into the stable native conformation. Both models predict partially folded intermediates in the folding pathway.

Compact kinetic intermediates have been found in the refolding of a number of single-domain proteins. These include $\alpha$-lactalbumin, ribonuclease $A$, apomyoglobin, cytochrome $c$, barnase, ubiquitin, and hen lysozyme. Kinetic intermediates comprises of "early" and "late" species. Early intermediates form rapidly in the millisecond time scale or faster, within deadtime of most techniques. Late intermediates accumulate prior to the ratedetermining step of folding, and can be well populated to be detected. Much of structural information of folding intermediates comes from study of the late intermediates. However, it is very difficult to obtain detailed structure characterizations of these kinetic intermediates due to their transient nature.

In the study of equilibrium unfolding of bovine $\alpha$-lactalbumin, a structural intermediate state was discovered (Kuwajima, et al., 1976). To describe the characteristics of this compact but with fluctuating intramolecular movement state, the term "molten globule" was proposed for this kind of folding intermediate state (Ohgushi \& Wada, 1983). Later, it was found that an early transient intermediate in the $\alpha$-lactalbumin refolding process had similar properties to this equilibrium molten globular state. Since then, evidence for identities between kinetic intermediates and equilibrium molten globule species has been found in several other proteins. A general role of molten globule forms involved on the folding pathway has thus been put forward (Kuwajima, 1989; Ptitsyn, 1995). 
Structural characterizations of these stable equilibrium intermediates provide insights into the mechanism of protein folding.

Lysozyme folding. Lysozyme is among one of the well-studied proteins in folding studies. The kinetic aspect of the folding processes has been examined by a variety of physical techniques, including stopped-flow fluorescence, far- and near-UV CD and absorption spectroscopies, hydrogen exchange labeling, nuclear magnetic resonance spectroscopy (NMR) and electrospray mass spectrometry (ESMS) (Dobson, et al., 1994). Information revealed from these complementary methods provides a rather detailed model for the folding pathway of lysozyme. Folding events along the pathway can be summarized to three stages. (1) At the earliest stage $(t<2 \mathrm{~ms})$, the unfolded states have collapsed into states with substantial secondary structure. However, the structure formed is not persistent, and therefore these states are highly dynamic and fluctuating. This early step is not resolved by any methods employed so far, and is inferred from the stopped-flow far-UV CD and fluorescence experiments. (2) In the next stage with time constant $\tau<100$ $\mathrm{ms}$, the collapsed states are interconverting and secondary structure is stabilized. Hydrogen exchange experiments have resolved different kinetic behaviors for the $\alpha$ and $\beta$ domains. A folding intermediate is implicated where the $\alpha$ domain has acquired persistent and native-like secondary structure, but the $\beta$ domain is still largely unstable (Radford, $e t$ al., 1992). The structure of the $\alpha$ domain at this stage resembles that of the molten globule state. (3) The native conformation is obtained at the last stage $(\tau-350 \mathrm{~ms})$. Structure in the $\beta$ domain is stabilized and $\alpha$ and $\beta$ domains are organized into the final native state. Note that these refolding experiments were performed at $\mathrm{pH} 5.3$.

In addition to these three stages, about $10 \%$ of the unfolded lysozyme molecules refolded in a slow process with time constant $\tau>16 \mathrm{~s}$ at pH 2.6 (Kato, et al., 1982). The slow process is attributed to the interconversion of proline isomers in the unfolded states. A ratio of trans to cis isomers in the unfolded states is thus derived to be $9: 1$.

Recently, the role of intermediates in the folding pathway has been challenged. From interrupted refolding experiments, a fast folding pathway of lysozyme has been identified with time constant $\tau \sim 50 \mathrm{~ms}$ (Baldwin, 1995; Kiefhaber, 1995). About 14\% molecules refold in this process, while the majority of molecules, $86 \%$, refold on a slower time scale that has been characterized as a three-stage process as described above. The two-state mechanism of the fast folding process suggests that the partially folded intermediates may be a result of kinetic traps in the folding pathway. 
Information regarding the compactness of folding molecules is normally derived from the binding of hydrophobic fluorescent probes such as 8-anilinonapthalene-1sulfonate (ANS), and intrinsic tryptophan fluorescence spectroscopy. However, these methods give only indirect information about protein compactness. Addition of ANS may complicate the already complex folding process; in fact, it has been found that ANS perturbs the refolding kinetics of lysozyme (Itzhaki, et al., 1994). Exclusion of solvent around tryptophan residues may not reflect the overall compaction of the folding process.

Clearly, direct structural techniques are needed to probe the compactness of the folding molecule in the folding process. Small-angle scattering (SAXS) is one of these techniques. The radius of gyration $\left(\mathrm{R}_{\mathrm{g}}\right)$ and distance distribution function $(\mathrm{P}(\mathrm{r}))$ derived from SAXS experiments, as described in detail in Chapter 1, give the measure of overall size and shape, thus compactness, of the protein molecule. Combined with the stoppedflow method, time-resolved SAXS can provide direct information on the kinetics of compactness in the folding process. The application of time-resolved SAXS technique to the study of lysozyme folding is described in this chapter.

\subsection{Experimental Procedures}

\subsubsection{Sample Preparations}

Hen egg-white lysozyme was purchased from the Sigma Chemical Company, St. Louis, MO. Lysozyme was directly dissolved in filtered $8 \mathrm{M}$ urea solutions in $100 \mathrm{mM}$ $\mathrm{NaCl}, 100 \mathrm{mM} \mathrm{Na}$-citrate buffer for $\mathrm{pH} 2.9$, or $100 \mathrm{mM} \mathrm{NaCl}, 100 \mathrm{mM}$ Na-phosphate buffer for $\mathrm{pH}$ 2.0. The solutions were centrifuged at 3,000 rpm in a Beckman Ti 50 rotor for 60 minutes at $15^{\circ} \mathrm{C}$. Unfolding was allowed to equilibrate for at least 5 hours before the scattering experiments. Dilution buffers were $100 \mathrm{mM} \mathrm{NaCl}, 100 \mathrm{mM}$ Na-citrate buffer at $\mathrm{pH} 2.9$ for the final $\mathrm{pH} 2.9$ condition, or $100 \mathrm{mM} \mathrm{NaCl}, 100 \mathrm{mM} \mathrm{Na}$-phosphate buffer at $\mathrm{pH} 2.5$ for the final $\mathrm{pH} 2.0$ condition. Buffers were filtered through a $0.2 \mu \mathrm{m}$ filter. The final lysozyme concentration was about $7 \mathrm{mg} / \mathrm{ml}$. All the urea-containing samples were used within 24 hours. 


\subsubsection{Data Collection}

Scattering data were collected at beamline 4-2 at Stanford Synchrotron Radiation Laboratory. The beamline configurations were basically the same as described in Section 4.2.2. A stopped-flow method was employed in the time-resolved scattering experiments. A stopped-flow rapid-mixer (Eliezer, 1994; Tsuruta, et al., 1989) was used to replace the sample cell of the equilibrium study. Experiments were carried out over three independent runs, June 94 , February 95 , and June 95 . Early data were collected using the rapid-mixer observation cell with $50 \mu \mathrm{m}$ quartz windows, while the latest data were collected with the same cell but with $40 \mu \mathrm{m}$ mica windows. The dilution ratios were 1:3.5 and 1:6 in volume. Refolding was initiated by rapidly diluting unfolded lysozyme in $8 \mathrm{M}$ urea to a buffer of $x M$ urea ( $x=0$, or $1 M$ depending on the mixing ratio), resulting in a strongly native condition ( $1-2 \mathrm{M}$ urea). Stopped-flow data were collected in various time frames, ranging from $20 \mathrm{~ms}$ to $2 \mathrm{~s}$, with a total time of $28 \mathrm{~s}$ as the longest period. A typical stopped-flow file contained data accumulated over 25 mixing repetitions. To improve the signal-to-noise ratio, hundreds of mixing events were measured for each condition. Control experiments were done by diluting sample solution (unfolded lysozyme in $8 \mathrm{M}$ urea) with $8 \mathrm{M}$ urea buffer without initiation of refolding, and data were collected in the same time-fashion as the corresponding stopped-flow experiments. The "initial" unfolded state was measured by diluting lysozyme in $8 \mathrm{M}$ urea to $8 \mathrm{M}$ urea buffer without invoking refolding, while the final folded state was measured by mixing lysozyme in $8 \mathrm{M}$ urea with appropriate buffer and allowing folding to proceed for at lease 1 minute before data collection. Both the "initial" state and the final state were characterized immediately before or after stopped-flow experiments using the same stopped-flow cell for direct $\mathrm{I}(0)$ comparison. Temperature was set at $20^{\circ} \mathrm{C}$ for most conditions used, however, experiments were also performed at $12^{\circ} \mathrm{C}$ and $30^{\circ} \mathrm{C}$. The $\mathrm{pH}$ of the final condition varied within 0.1 unit.

\subsubsection{Data Analysis}

Data reduction. A typical stopped-flow experiment consisted of tens of data files totaling hundreds of mixing repetitions. Data of each stopped-flow file (25 mixing repetitions) were first normalized, background subtracted and calibrated. The calibrated data were carefully examined among files before they were combined together. It is not uncommon that data taken within the same time block (normally 6 hours) had to be grouped into two or even three sets. Time-dependence of parasitic scattering was apparent when 
comparing the background data taken in the same block. For some unknown reason, a shift of scattering pattern by a few channels was also observed among the stopped-flow sample data, and between the stopped-flow and the static (background) data. For those time-dependent data sets, data were first divided into groups, and an appropriate background for each group was found to be either the background file taken immediately before or after the data group or a linear combination of these two background files. Data from different groups were not always combined. For data displaying a channel-shift, in some cases it was not possible to find the displacement, thus the stopped-flow sample data could not be combined or the sample data and the background data could not match. Data were not further analyzed in these cases. Fortunately, there were only a few files with this kind of anomaly.

Guinier analysis. Radii of gyration were derived from the Guinier approximation :

$$
\operatorname{LnI}(S)=\operatorname{LnI}(0)-\frac{4 \pi^{2}}{3} S^{2} R_{g}^{2}
$$

The fitting region, $0.0045-0.001 \AA^{-1}$ in $\mathrm{S}$, for all the analysis including the stopped-flow and the static data sets was chosen to be the same as that in the equilibrium unfolding studies described in Chapter 4. The forward scattering intensity, $\mathrm{I}(0)$, was also obtained from equation [1].

To estimate the correction factor for $\mathrm{I}(0)$ due to changes in solvent (urea) contrast, the electron density of lysozyme is approximated as follows. The partial specific volume, molecular weight and number of total electrons of lysozyme are $0.703 \mathrm{~cm}^{3} / \mathrm{g}, 14,600$, and 7,700 , respectively. Then, the electron density of Lysozyme is:

$$
[7,700 /(14,600 \times 0.703)] \times 6.02 \times 10^{23} / 10^{24}=0.45\left(\mathrm{e}^{-} / \AA^{3}\right)
$$

The concentration of $\mathrm{H}_{2} \mathrm{O}$ is $55.55 \mathrm{M}$. Since the density of urea is $1.323 \mathrm{~g} / \mathrm{cm}^{3}$, the amount of $\mathrm{H}_{2} \mathrm{O}$ displaced by urea in a $\mathrm{n} \mathrm{M}$ urea solution is $45.35 \mathrm{n} \mathrm{g} /$, or $2.52 \mathrm{n} \mathrm{mol} /$. The number of electrons in one molecule of $\mathrm{H}_{2} \mathrm{O}$ and urea is 10 and 32, respectively. The electron density of $\mathrm{n} \mathrm{M}$ urea in $\mathrm{H}_{2} \mathrm{O}$ solution is then:

$$
[10 \times(55.5-2.52 n)+32 n] \times 10^{-3} \times 6.02 \times 10^{23} / 10^{24}=0.334+0.00409 n\left(e / \AA^{3}\right)
$$


The increase in electron density due to the presence of buffer $100 \mathrm{mM} \mathrm{NaCl}, 100 \mathrm{mM} \mathrm{Na}$ citrate is about $0.0095 \mathrm{e}^{-/} / \AA^{3}$. Therefore, the ratio of $\mathrm{I}(0)$ of lysozyme in $1 \mathrm{M}$ urea (one of the final refolding conditions) to that in $8 \mathrm{M}$ urea is $[(0.45-0.347) /(0.45-0.376)]^{2}=1.9$, so the $\mathrm{I}(0)$ in the refolding is about twice that of the control.

Kinetic analysis. The kinetics of changes in $R_{g}$ as a function of time was fit into single exponential as formulated in equation [2]:

$$
R_{g}^{2}(t)=A e^{-k t}+R_{g}^{2}(e q)
$$

where $R_{g}{ }^{2}(t)$ is the $R_{g}{ }^{2}$ value measured at the refolding time $t, R_{g}{ }^{2}(e q)$ is the equilibrium value of $\mathrm{R}_{\mathrm{g}}{ }^{2}$ corresponding to the final native state, $k$ is the rate constant (later referred to as time constant $\tau, \tau=1 / k$ ), and $A$ is the amplitude. The refolding process could also be fit with two exponential terms. However, the quality of the current data did not allow a clear distinction between these two fitting procedures. In addition, the doubleexponential approximation did not give consistent results for independent experiments performed under the same conditions. Therefore, the refolding process was treated as a one-step kinetic process and approximated with a single exponential.

\subsection{Results}

\subsubsection{Sample Integrity}

No evidence of nonspecific sample aggregation was observed for all the samples studied, including both the initial and final states and during the refolding kinetics, since if present it would show up in the small angle region in the Guinier plot. No clear depressive trend was displayed in the Guinier region, agreeing with the conclusion in Section 4.4.2 that interparticle interference can be ignored at this lysozyme concentration $(7-8 \mathrm{mg} / \mathrm{ml})$. For the static measurements on the initial and final states, data were collected up to 10 minutes and no time dependence in scattering pattern was observed. Since the longest data collection time in the stopped-flow experiments was 28 seconds, radiation-induced sample degradation for all samples can therefore be excluded. 


\subsubsection{The Initial and Final States}

In all cases, $\mathrm{R}_{\mathrm{g}}$ of lysozyme at the initial conditions ( $8 \mathrm{M}$ urea at $\mathrm{pH} 2.9$ or $\mathrm{pH} 2.0$ ) from both the static and control experiments was found to be about $22 \AA$, indicative of an unfolded state of lysozyme at the beginning of the refolding experiments. The association state of lysozyme at the initial conditions can be inferred from a comparison of $\mathrm{I}(0)$ values of the initial and final states. For $\mathrm{pH} 2.9$, a ratio of 1.83 is obtained between $1 \mathrm{M}$ urea and $8 \mathrm{M}$ urea samples, comparable to the predicted factor (1.9) due to a change in solvent contrast. The consistency in $I(0)$ after correction for solvent contrast suggests that the unfolded lysozyme is largely monomeric in $8 \mathrm{M}$ urea. Further supporting argument comes from the stopped-flow experiments and the control experiments. Fig. 5.1 shows the scattering curves of the last time-frame of the stopped-flow data and the corresponding control. Again, a factor of about 2 for $I(0)$ is derived, suggesting the monomeric state of the unfolded lysozyme in the initial condition. $I(0)$ for the initial and final states are comparable to those obtained for the control and the stopped-flow data, respectively, although values from the static data are systematically higher than those from the timeresolved data by $15 \%$.

At the end of refolding, $R_{\mathrm{g}}$ of lysozyme $(\sim 15.3 \AA)$ is in good agreement with that of the native state determined in Section 4.4.2. In addition, scattering curves of the last few time-frames superimpose with that of the final state, taken 1 minute after initiation of refolding. Therefore, within SAXS resolution, lysozyme has completely refolded to the folded state by the end of the refolding process followed by these time-resolved SAXS experiments.

\subsubsection{The Refolding Kinetics}

Several attempts were made to probe the early events in the refolding process by inducing the process at a lower temperature, or by refolding to a higher urea concentration. The time duration at the beginning of data collection were tried with $20 \mathrm{~ms}, 50 \mathrm{~ms}$ and 100 $\mathrm{ms}$, and the relative signal-to-noise ratios for the $20 \mathrm{~ms}$ and $100 \mathrm{~ms}$ time-frame can be seen in Fig. 5.2. A deadtime of about $13 \mathrm{~ms}$ was estimated with the current SAXS setup; the deadtime includes both the inherent deadtime of the stopped-flow mixer and the time delay due to signal transfer among the computer, various electronic modules and the mixer. To obtain a reliable kinetic analysis on changes in $\mathrm{R}_{\mathrm{g}}$, the current smallest duration time-frame was found to be $100 \mathrm{~ms}$ with an accumulation of a few hundreds mixing repetitions. The 


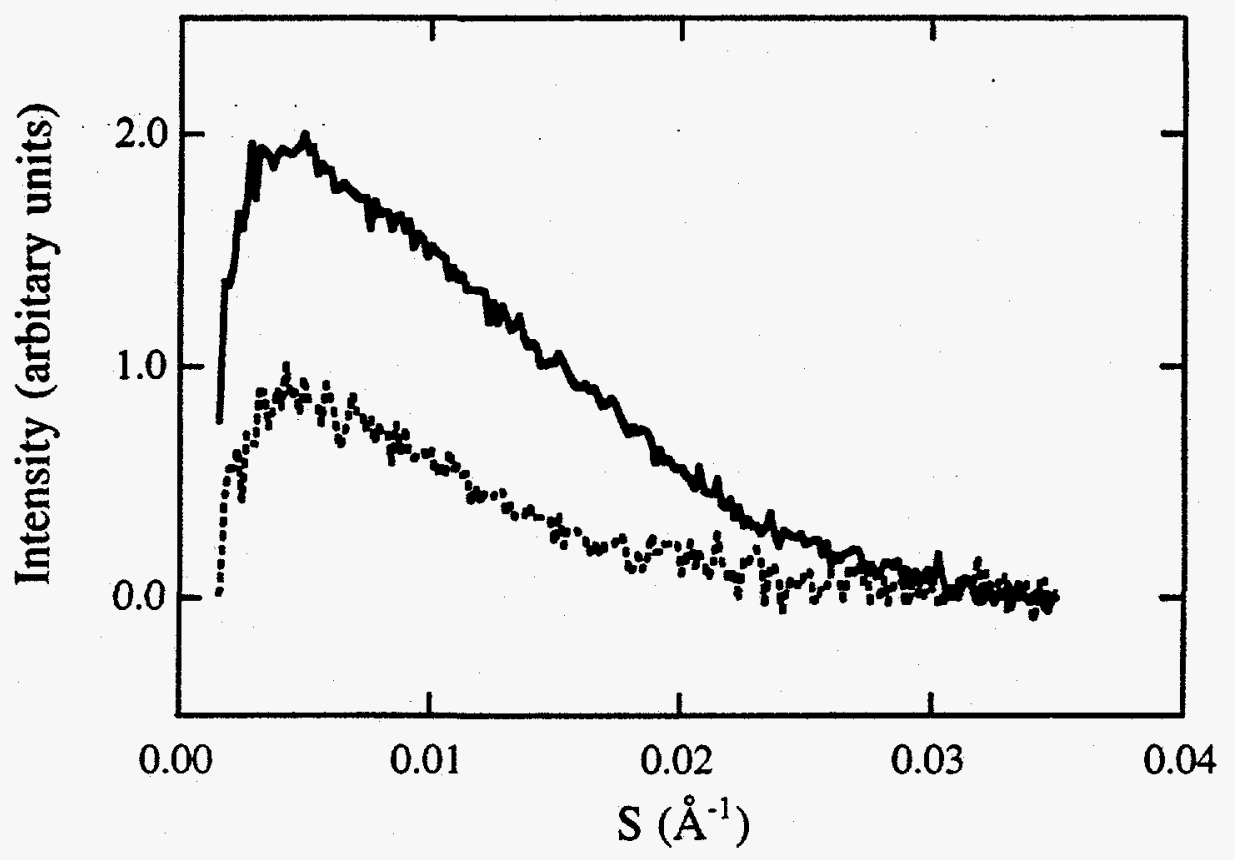

Figure 5.1. Scattering curves of the last time-frame of a stopped-flow ( - ) and the corresponding control experiments (- - ). The duration time is $2 \mathrm{~s}$, and the number of mixing events is 700 . The final refolding condition is $1.8 \mathrm{M}$ urea. $\mathrm{I}(0)$ of the unfolded state (in the control data) is about half of $\mathrm{I}(0)$ of the refolded state (the final stage in the stopped-flow data). 


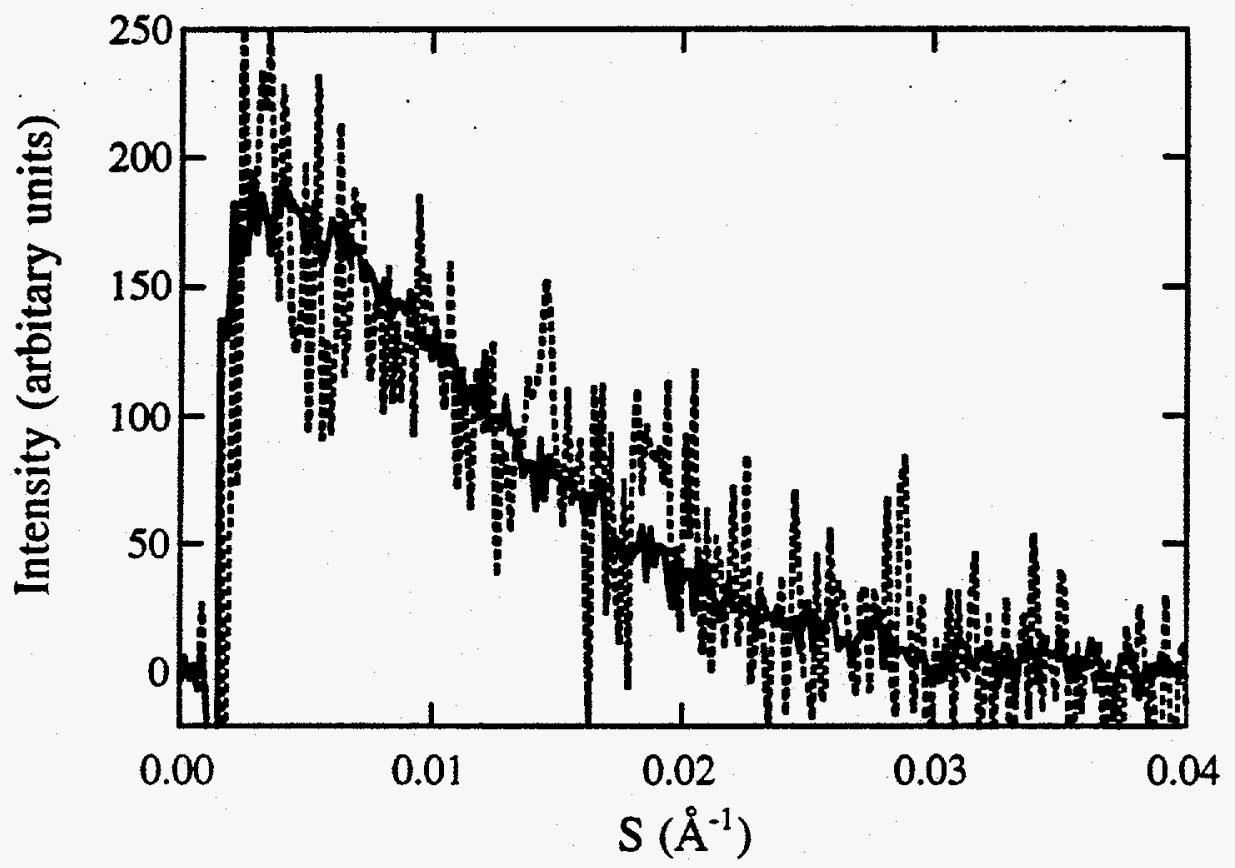

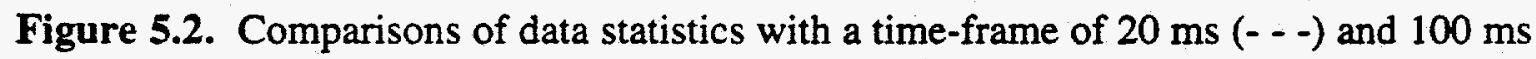
(一). The accumulation is 305 mixing events. Data with $20 \mathrm{~ms}$ duration time is apparently too noisy for Guinier analysis. 
use of more intense beam will certainly improve the statistics of $20 \mathrm{~ms}$ time frame and thus time resolution, as well as reduce the number of repetitions which becomes an important factor when experiments are constrained by sample supply .

Fig. 5.3. represents a typical time-course of $R_{g}$ in the refolding with a final condition of $1.8 \mathrm{M}$ urea at $\mathrm{pH} 2.9$. At the earliest resolved time $(\sim 100 \mathrm{~ms}$ after initiation of refolding), $R_{g}$ is found to be $-19 \AA$. $A R_{g}$ of $\sim 15 \AA$ is reached about $5 \mathrm{~s}$ after the refolding, and is stabilized over the rest period of the collection time, suggesting that the major refolding kinetic events is complete within 5 seconds. Note that the initial state of lysozyme is verified to be in the unfolded state with a $R_{g}$ of $\sim 22 \AA$ (see Section 5.3.2). Further, a $\mathrm{Rg}$ consistent with that of the unfolded state is obtained from the control data. The lack of time dependence of $\mathrm{R}_{\mathrm{g}}$ in the control experiment (see Fig. 5.4) strongly suggests that the substantial reduction in $\mathrm{Rg}$ observed at the very first $100 \mathrm{~ms}$ of refolding is not due to a dilution from a relative concentrated lysozyme solution $(\sim 32$ or $\sim 56 \mathrm{mg} / \mathrm{ml})$ to a final $\sim 8 \mathrm{mg} / \mathrm{ml}$ condition. The time-course of $\mathrm{Rg}$ was fit with a single exponential according to equation [2], and is shown in Fig. 5.3. However, the single-phase kinetics does not account for the total amplitude; a loss of $\sim 30 \%$ of the total change in $\mathrm{R}_{\mathrm{g}}$ occurs within the burst phase $(<100 \mathrm{~ms})$. Therefore, this indicates that there is a faster phase occurring prior to the slower phase seen in time-resolved SAXS data. This result implies the existence of an early intermediate in the pathway.

The derived time constants from different experiments and under various refolding conditions are presented in Table 5.1. Refolding to a final condition of $1 \mathrm{M}$ urea at $\mathrm{pH} 2.9$. was repeated several times, and a time constant of $\sim 1 \mathrm{~s}$ is consistently obtained from the data. As expected, refolding proceeds slower for transitions to $2 \mathrm{M}$ than to $1 \mathrm{M}$ (by a factor of 2). The refolding rate is further reduced again by about a factor of 2 with a decrease of $8{ }^{\circ} \mathrm{C}$ in temperature. The slowest process, $\sim 9 \mathrm{~s}$, is observed with transition to final urea concentration of $2 \mathrm{M}$ at $\mathrm{pH} 2.0$, a less favorable native condition just outside the transition region as seen in Fig. 4.11.b.

Fig. 5.5 shows the Kratky plots of the initial and the final time-frames. The presence of a peak in the Kratky plot indicates that at the early stage of refolding $(\sim 100$ $\mathrm{ms}$ ), lysozyme has collapsed into a globular structure. It is not surprising given that even in $8 \mathrm{M}$ urea, lysozyme is not completely unfolded to a random coil structure as suggested by trace evidence of a peak in the Kratky plot (see Fig. 4.9.a). The well defined peak from the last time-frame is a characteristic of a globular structure. 

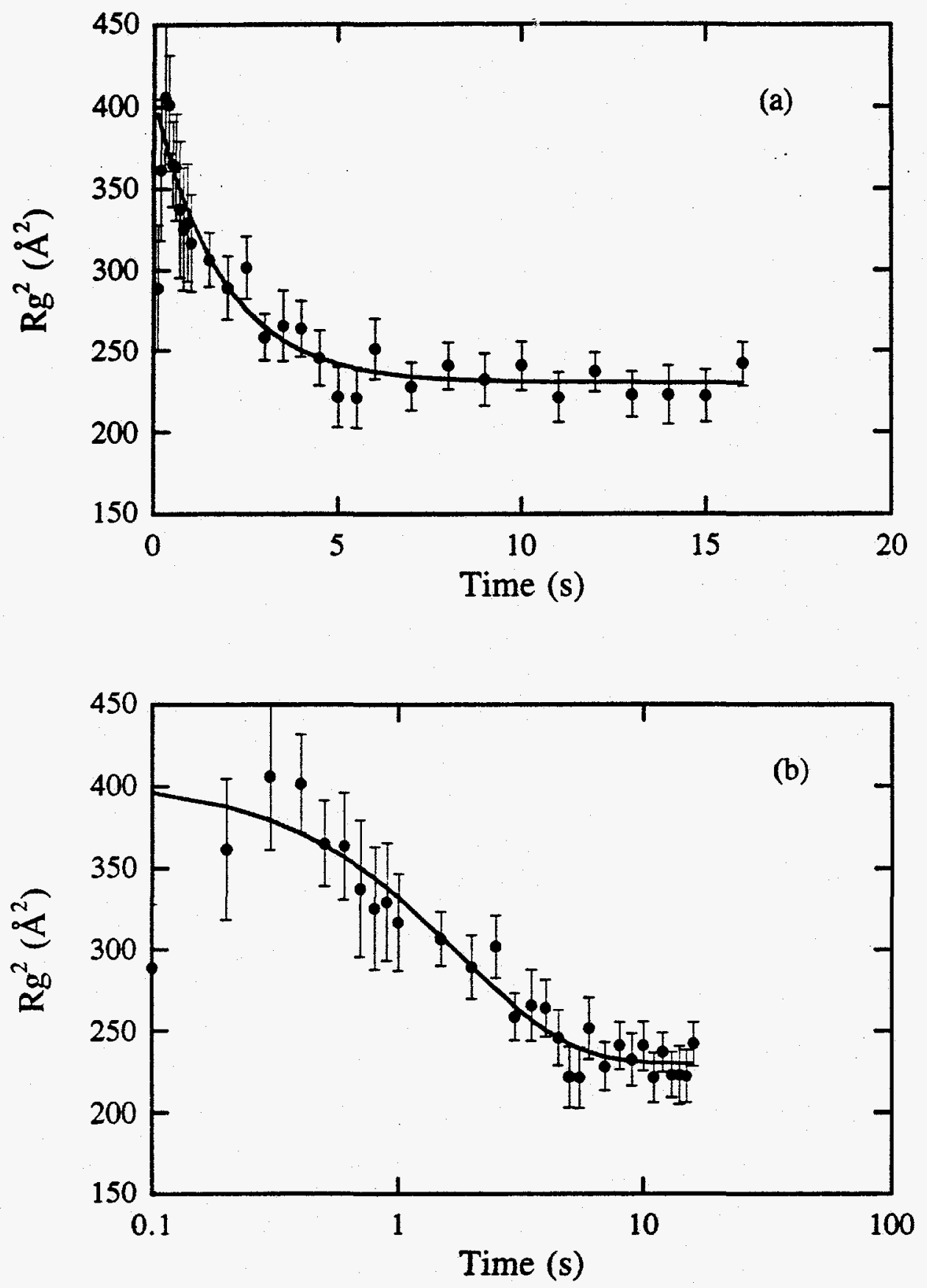

Figure 5.3. Time-course of $\mathrm{Rg}^{2}$ in the refolding of lysozyme at $\mathrm{pH}$ 2.9. The transition is from $8 \mathrm{M}$ urea to $1.8 \mathrm{M}$ urea. (a) time is in linear scale; (b) time is in log scale for a better display of the early events. 


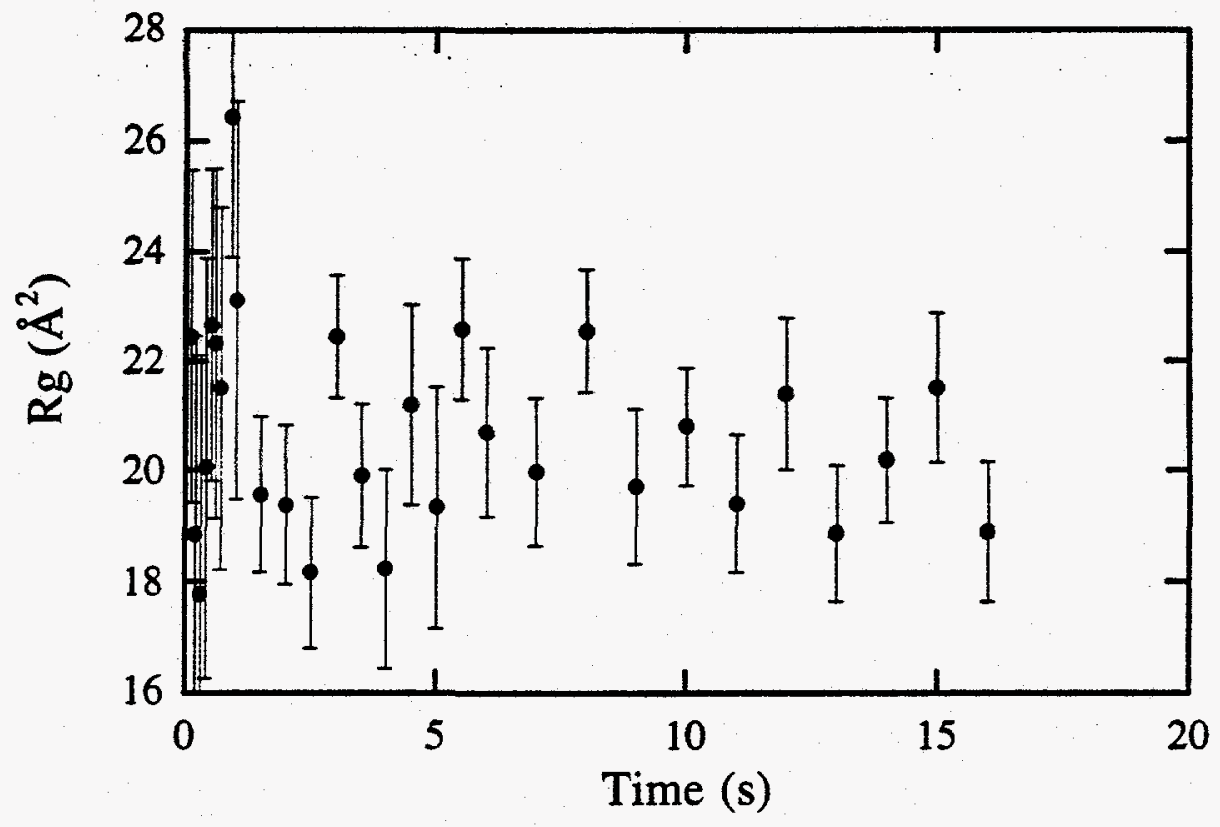

Figure 5.4. Time-course of $R_{g}$ in a control experiment. The control experiment was done by diluting unfolded lysozyme in $8 \mathrm{M}$ urea to a $8 \mathrm{M}$ urea buffer without initiating 'refolding. $\mathrm{Rg}$ is steadily about $21 \AA$, comparable to that of the unfolded state from static measurements. The decrease in $\mathrm{Rg}$ during the refolding process is not observed in the control. . 
Table 5.1. Kinetic parameters derived for lysozyme refolding.

\begin{tabular}{lccccc}
\hline $\begin{array}{c}\text { final refolding } \\
\text { conditions }\end{array}$ & $\begin{array}{c}\text { rate constant } \\
\mathrm{k}\left(\mathrm{s}^{-1}\right)\end{array}$ & $\begin{array}{c}\text { time constant } \\
\tau(\mathrm{s})\end{array}$ & $\begin{array}{c}\mathrm{R}_{\mathrm{g}}(\AA) \text { for } \\
\text { the final state }\end{array}$ & amplitude & $\begin{array}{c}\text { numbers } \\
\text { of } \\
\text { repetitions }\end{array}$ \\
\hline $1 \mathrm{M}$ urea, $\mathrm{pH}$ & $0.97( \pm 0.1)$ & $1.03( \pm 0.11)$ & $15.6( \pm 0.1)$ & $132.3( \pm 7.1)$ & $265^{\mathrm{b}}$ \\
$2.9,20^{\circ} \mathrm{C}$ & & & & & \\
& $1.38( \pm 0.2)$ & $0.72( \pm 0.11)$ & $15.6( \pm 0.1)$ & $133.8( \pm 9.2)$ & $400^{\mathrm{b}}$ \\
& $0.91( \pm 0.15)$ & $1.1( \pm 0.18)$ & $15.0( \pm 0.1)$ & $128.7( \pm 10.1)$ & $150^{\mathrm{b}}$ \\
& $1.0( \pm 0.03)$ & $1.0( \pm 0.03)$ & $15.5( \pm 0.1)$ & $124.4( \pm 1.8)$ & $225^{\mathrm{b}}$ \\
\hline $2 \mathrm{M}$ urea, $\mathrm{pH}$ & $0.54( \pm 0.05)$ & $1.85( \pm 0.17)$ & $15.2( \pm 0.1)$ & $175.3( \pm 6.0)$ & $725^{\mathrm{a}}$ \\
$2.9,20^{\circ} \mathrm{C}$ & & & & & \\
\hline $2 \mathrm{M}$ urea, $\mathrm{pH}$ & $0.25( \pm 0.1)$ & $4.0( \pm 1.6)$ & $15.6( \pm 0.4)$ & $114( \pm 15.6)$ & $405^{\mathrm{a}}$ \\
$2.9,12{ }^{\circ} \mathrm{C}$ & & & & & \\
\hline $2 \mathrm{M}$ urea, $\mathrm{pH}$ & $0.11 \pm 0.02)$ & $9.1( \pm 1.65))$ & $14.7( \pm 0.3)$ & $130.6( \pm 8.8)$ & $705^{\mathrm{a}}$ \\
$2.0,20^{\circ} \mathrm{C}$ & & & & & \\
\hline
\end{tabular}

a data taken with monochromatic beam.

$\mathrm{b}$ data taken with multilayer beam. 


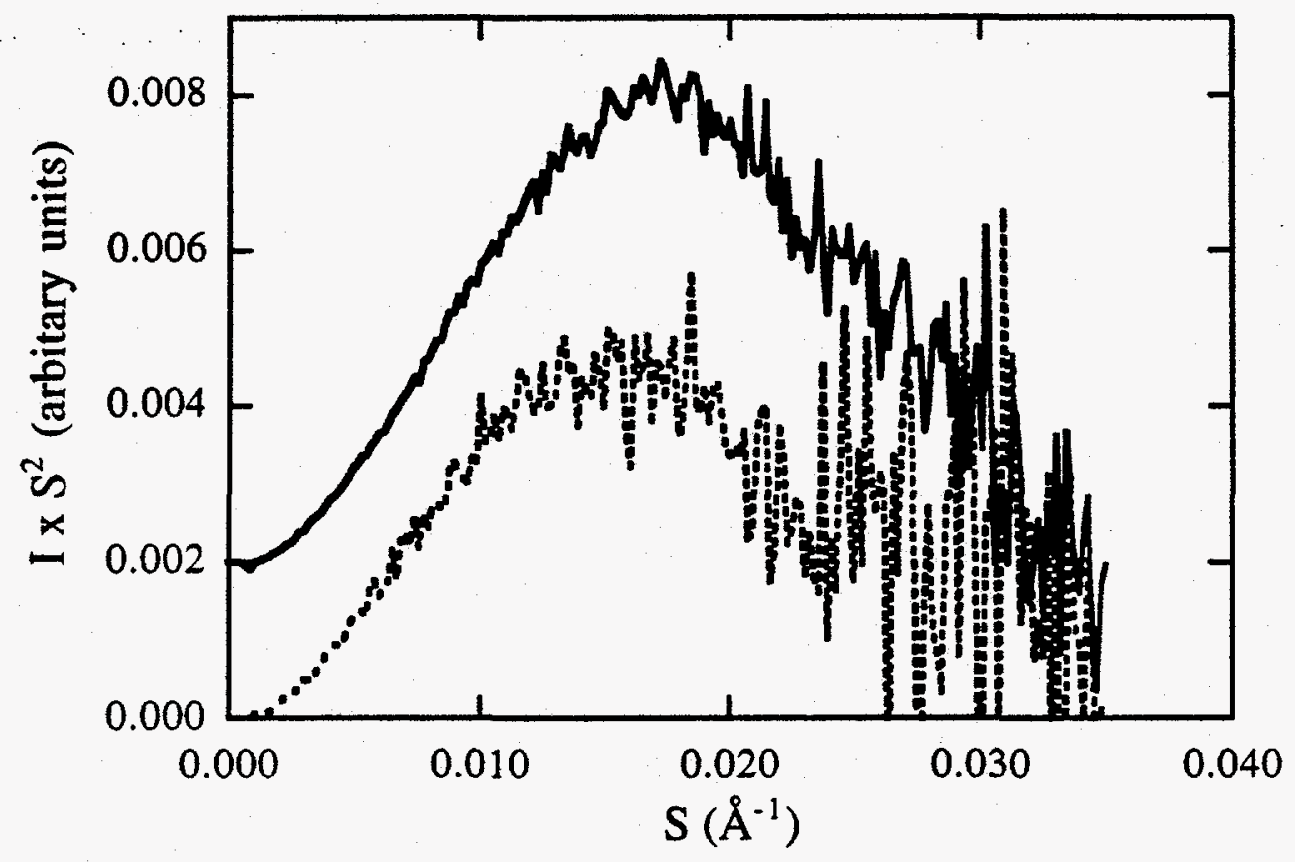

Figure 5.5. Kratky plots of the first $100 \mathrm{~ms}$ time-frame data (- - ) and the last $2 \mathrm{~s}$ timeframe data (-). The stopped-flow process was followed for 16 seconds. For clarity, Kratky plot of the $2 \mathrm{~s}$ duration data is shifted by 0.002 unit. The accumulation is 700 mixing events. 
Immediately following the mixing, the background buffer remains the same throughout data collection. Consequently, $\mathrm{I}(0)$ should stay steady in the refolding process. $\mathrm{I}(0)$ values from the stopped-flow data are reasonably consistent with a variation of 10 $15 \%$, a greater deviation than normally observed in the static measurements. The mean $\mathrm{I}(0)$ is about $15 \%$ lower than $\mathrm{I}(0)$ of the final state in static measurement. (The mean $\mathrm{I}(0)$ of the control data is also $15 \%$ smaller than the corresponding $\mathrm{I}(0)$ of the initial state in the static measurement, see Section 5.3.2.) On the other hand, Rgs derived from time-resolved and static experiments agree well, and are reproduced between independent measurements. The reason for the inconsistency in $I(0)$ is not clear so far. However, it might provide an estimate to the magnitude of deviation in $\mathrm{I}(0)$.

For some data sets, although noisy, a change in $\mathrm{I}(0)$ is observed for the first few time frames, including a step decrease of $\sim 15 \%$. Since the magnitude of the difference is comparable to that of the $I(0)$ deviation, an interpretation of the change might not be meaningful. Despite a discrepancy in $\mathrm{I}(0)$ derived from the Guinier plot, scattering from the first and the last time-frames looks similar as seen in Fig. 5.5. The difference in $\mathrm{I}(0)$ derived from the Guinier plot might be attributed to the fitting error. Nor can it be excluded that the change is real. Better data quality and more measurements are necessary to justify a conclusive analysis. At the current stage, allowing a $15 \%$ deviation in $\mathrm{I}(0)$ derived from the Guinier plot, it appears that the association state of at least the majority of the lysozyme does not change throughout the refolding process, and is largely monomeric. The change in $\mathrm{Rg}$ can thus be correlated with conformational changes, rather than with a change in association state as observed in the case of myoglobin (Eliezer, et al., 1993), of lysozyme in the refolding.

\subsection{Discussion}

Our time-resolved SAXS experiments provide direct measurements of compaction process in the folding pathway. At the earliest measurable time, $100 \mathrm{~ms}$ after initiation of refolding, $R_{g}$ has decreased from $\sim 22 \AA$ of the unfolded state to $\sim 19 \AA$. A single exponential fit to the data can not cover the total amplitude, suggesting that a faster separate process occurs within the deadtime (100 ms) of current experiment. Within experimental errors, $\mathrm{I}(0)$ remains consistent throughout the refolding, and is comparable to a monomeric 
lysozyme. Therefore, the observed kinetics of changes in $\mathrm{R}_{\mathrm{g}}$ corresponds to the timecourse of conformational change of lysozyme in the refolding process.

For hen lysozyme refolding at $20^{\circ} \mathrm{C}$ to a final concentration of $0.5 \mathrm{M} \mathrm{GdmHCl}$ at pH 5.2 (Itzhaki, et al., 1994), two kinetic phases are observed in the binding of ANS with time constants of $\tau_{1}=23 \pm 5 \mathrm{~ms}$ and $\tau_{2}=250 \pm 60 \mathrm{~ms}$, and in the intrinsic tryptophan fluorescence measurement with time constants of $\tau_{1}=25 \pm 3 \mathrm{~ms}, \tau_{2}=340 \pm 20 \mathrm{~ms}$. Our kinetics of changes in $\mathrm{Rg}_{\mathrm{g}}$ is also biphasic. The burst phase, not resolved within our experimental deadtime of $100 \mathrm{~ms}$, can be correlated with the fast process of ANS binding and tryptophan fluorescence, or the second stage of the folding event as described in Section 5.1. A substantial compaction occurs at this initial step with about 2-3 $\AA$ decrease in $\mathrm{Rg}$. The second process ( $\tau=1 \mathrm{~s}$ for the final condition of $1 \mathrm{M}$ urea at $\mathrm{pH} 2.9$ ) may also be correlated to the slow phase observed in ANS binding and intrinsic tryptophan fluorescence experiments based on the following reasons. (1) The refolding conditions used in our SAXS study are less favorable for folding. The ANS binding and intrinsic tryptophan fluorescence measurement were performed at a strongly native folding condition at $\mathrm{pH} 5.2$, while SAXS experiments described here were carried out at $\mathrm{pH} 2.9$ and $\mathrm{pH} 2.0$. As expected, at a lower $\mathrm{pH}$ condition, the folding process will definitely slow down. A time constant of $1.3 \mathrm{~s}$ has been reported for lysozyme folding to a final concentration of 0.5 $\mathrm{M} \mathrm{GdmHCl}$ at $\mathrm{pH} 2.6$ at $25^{\circ} \mathrm{C}$ (Kato, et al., 1982). In our experiment, the refolding to a final concentration of $1.8 \mathrm{M}$ urea at $\mathrm{pH} 2.0$ is much slower, with $\tau=9.1 \pm 1.6 \mathrm{~s}$ (see Table 5.1). In addition, denaturants also affect folding. At pH 2.6 and $25^{\circ} \mathrm{C}$, refolding rate decreases with the increase of $\mathrm{GdmHCl}$ concentration, $\tau=1.3,4.3$ and $13.9 \mathrm{~s}$ for $0.5 \mathrm{M}$, $1.0 \mathrm{M}$ and $1.5 \mathrm{M} \mathrm{GdmHCl}$, respectively (Kato, et al., 1982). The kinetics of changes in $\mathrm{R}_{\mathrm{g}}$ is slower by a factor of 2 when refolding to a final concentration of $1.8 \mathrm{M}$ urea than refolding to a final concentration of $1 \mathrm{M}$ urea solution (see Table 5.1). (2) Although ANS binding and tryptophan fluorescence measurements suggest a time constant of $\sim 300 \mathrm{~ms}$ for the last compaction process, hydrogen protection of some residues, mostly located in the $\beta$ domain, occurs in a much slower phase $\sim 400-800 \mathrm{~ms}$ (Radford, et al., 1992). This suggests that the overall compactness in the folding process may not be complete at the time of the exclusion of ANS and the protection of tryptophan residues. A longer process observed by $R_{g}$ is therefore not unexpected.

A folding intermediate was identified mainly by hydrogen exchange experiments in the second stage of refolding with $\tau<100 \mathrm{~ms}$ (see Section 5.1). The $\alpha$ domain of the folding intermediate has molten globule features, with persistent native-like secondary 
structure and a loose tertiary fold, but the $\dot{\beta}$ domain is still largely unstructured. The biphasic kinetics observed by our time-resolved SAXS suggests that a folding intermediate has formed within $100 \mathrm{~ms}$ after initiation of refolding. The time scale of formation of this intermediate corresponds well with that of the intermediate reported previously, suggesting that these two species may be the same, i.e., the intermediate found by SAXS has substantial native secondary structure in a distorted $\alpha$ domain, and an unstable $\beta$ domain.

A stable intermediate has been resolved in the equilibrium unfolding study by SAXS (see Chapter 4) with $R_{g}$ of 19-20 $\AA$. The stable intermediate has a similar structure to that identified in the second stage of refolding. A rough estimate of $R_{\mathbf{g}}$ for the folding intermediate after the initial burst phase is about $19( \pm 1) \AA$ (Table 5.1). The similar $R g$ values imply that the stable intermediate might resemble the folding intermediate in the refolding pathway.

\subsection{Acknowledgments}

I would like to thank Dr. Hirotsugu Tsuruta and Daniel Segel for the assistance in data acquisition. This research was supported by National Institutes of Health Grant RR01209 (to K.O.H.). Data were collected at beam line 4-2 at SSRL which is supported by the U.S. Department of Energy, Office of Basic Energy Sciences, and in part by the National Institutes of Health, Biomedical Research Technology Program, National Center for Research Resources and by the Department of Energy, Office of Health and Environmental Research.

\subsection{References}

Baldwin, R. L. (1995). J. Bio. NMR, 5, 103-109.

Dobson, C. M., Evans, P. A., \& Radford, S. E. (1994). Tibs, 19, 31-37.

Eliezer, D. (1994) Protein folding and protein metallocluster studies using synchrotron small angle $x$-ray scattering. $P h . D$. Thesis, Stanford University.

Eliezer, D., Chiba, K., Tsuruta, H., Donlach, S., Hodgson, K. O., \& Kihara, H. (1993). Biophysical J., 65, 912-917.

Itzhaki, L. S., Evans, P. A., Dobson, C. M., \& Radford, S. E. (1994). Biochemistry, 33, 5212-5220. 
Karplus, M., \& Weaver, D. L. (1976). Nature, 260, 404-406.

Kato, S., Shimamoto, N., \& Utiyama, H. (1982). Biochemistry, 21, 38-43.

Kiefhaber, T. (1995). Proc. Natl. Acad. Sci. USA, 92, 9029-9033.

Kim, P. S., \& Baldwin, R. L. (1990). Annu. Rev. Biochem., 59, 631-660.

Kuwajima, K. (1989). Proteins, 6, 87-103.

Kuwajima, K., Nita, K., Yoneyama, M., \& Sugai, S. (1976). J. Mol. Biol., 106, 359373.

Ohgushi, M., \& Wada, A. (1983). FEBS, 164, 21-24.

Ptitsyn, O. B. (1973). Dokl. Acad. Nauk SSSR, 210, 1213-1215.

Ptitsyn, O. B. (1995). Molten globule and protein folding. In Anfinsen, C. B., Edsall, J. T., Richards, F. M., \& Eisenberg, D. S. (Eds.), Advances in Protein Chemistry (pp. 83-230). San Diego: Academic Press, Inc.

Radford, S. E., Dobson, C. M., \& Evans, P. A. (1992). Nature, 358, 302-307.

Schmid, F. X. (1992). Kinetics of unfolding and refolding of single-domain proteins. In Creighton, T. C. (Eds.), Protein Folding (pp. 197-242). New York: W. H. Freeman and Company.

Tsuruta, H., Nagamura, T., Kimura, K., Igarashi, Y., Kajita, A., Wang, Z.-X., Wakabayashi, K., Amemiya, Y., \& Kihara, K. (1989). Rev. Sci. Instrum., 60, 2356-2358. 